\title{
The coupling of waves and convection
}

\author{
Andrew Peter Stamp \\ Research School of Earth Sciences
}

\begin{abstract}
A thesis submitted for the degree of
Doctor of Philosophy

of The Australian National University
\end{abstract}

March 31, 1994

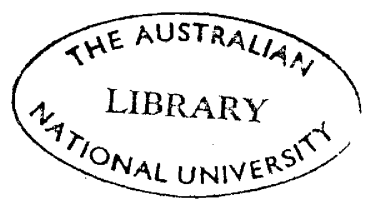




\section{Statement}

The work described in this thesis was carried out while I was a full-time research student in the Geophysical Fluid Dynamics Group at the Research School of Earth Sciences, The Australian National University. Except where mentioned in the acknowledgments, the research is my own. No part of this thesis has been submitted to any other university.

Andrew Peter Stamp

March 1994, Canberra

... A.ศ. Ktap.............. 


\section{Acknowledgments}

I thank my supervisors Dr Ross Griffiths and Professor Stewart Turner for their encouragement and advice throughout this study. Ross has challenged and inspired with his deep understanding of fluid mechanics and has been a patient friend, while Stewart's insightful comments over morning tea and willingness to share ideas has provided an ideal environment for research.

This study would not have been possible without the continual technical support of Messieurs Derek Corrigan, Tony Beasley and Ross Wylde-Browne. I am especially thankful to Derek who survived my visits to the laboratory and shared his intuitive ability to design elegant experiments. Dr Doug Christie and Messieurs Marcus Jacka and Matthew Stocks also assisted with the solitary wave work.

I appreciate the support of the other RSES staff and students. Dr Scott Condie has wielded his red pen and sense of humour, just has Dr Ross Kerr has wielded his tennis racket. Thanks to efforts of Drs Geoff Davies and Ian Campbell the abbreviation MORB is no longer a mystery and I can spell words like 'petrogenesis'. Stephen Pell has been a good friend with whom to share the daily trials and tribulations of research.

The companionship of Janine Kirkup has made the dark moments light, and my flatmates Jeremy Kirk and Loretta Power have been most supportive. Jeremy's advice with grammer have had a good affect on my writing style, and Loretta's courageous purchase of a wok changed the way I think of food. 


\section{Abstract}

This dissertation demonstrates that coupling of large-amplitude waves and turbulent convection, driven by fluxes through a diffusive interface, is readily established in the laboratory. The interaction of waves and convection may also be important for other systems such as atmospheric squall lines and the coupling of fluctuations in atmospheric circulation with long waves on the equatorial ocean thermocline.

To understand the behaviour of the waves without convection, experimental measurements were made of solitary waves propagating along a slightly diffused density interface between two deep motionless layers of constant density. Small- and largeamplitude waves behaved differently; small waves carried energy and momentum, whereas sufficiently large waves also carried mass. In addition, the wavelength of small-amplitude waves decreased with increasing amplitude, whereas for largeamplitude waves the wavelength increased with increasing amplitude. However, the wavespeed increased linearly with increasing amplitude for the entire range of amplitudes. The head-on collision between waves of equal amplitudes was similar to a reflection from a solid vertical boundary, whereas for waves of different amplitudes there was an exchange of fluid such that the leftward and rightward propagating waves had the same amplitudes before and after the collision.

The coupling of interfacial waves with convection was then investigated in detail. Experiments showed that when layers of salt and sugar solution are separated by a diffusive interface, interfacial waves are spontaneously generated by the convection once the system evolves to a critical value of the density-anomaly ratio $R_{\rho} \equiv \beta \Delta S / \alpha \Delta T$. The waves corresponded to a local thickening of the interface and produced horizontal variations in the interfacial buoyancy flux, which in turn forced circulations on the scale of the distance between waves. Subsequent interactions between the waves and convection led to a rapid decrease in the number of waves and an increase in the scale of the convective circulations. In rectangular channels a wide range of conditions gave rise to a single wave which propagated back-andforth, organizing the convection into tank-scale circulations that reversed direction quasi-periodically. The dependence of the wavespeed on the layer properties and channel dimensions was successfully predicted by assuming that coupling requires a matching of the wave and convection speeds, and that the system selects waves of an amplitude for which this resonance can occur.

Similar experiments in an annulus revealed convection cells of a travelling-wave nature, coupled to the waves on the interface. Thus reflection from endwalls was not required. Waves and reversals of convective circulations also developed in systems of multiple interfaces.

These strongly nonlinear phenomena involving large-amplitude waves and turbulent convection are worthy of further investigation as their role in Nature is not yet clear. Laboratory experiments play an important role in understanding these flows because mathematical models are difficult to formulate and numerical models require high resolution. 


\section{Contents}

1 Introduction 1

1.1 Research approach $\ldots \ldots \ldots \ldots \ldots \ldots \ldots$

2 Deep-water internal solitary waves $\quad 7$

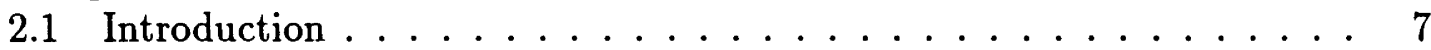

2.2 Review of the literature $\ldots \ldots \ldots \ldots \ldots$

2.2 .1 Overview of internal solitary wave theory . . . . . . . . 9

2.2.2 Deep-water internal solitary waves $\ldots \ldots \ldots \ldots \ldots$

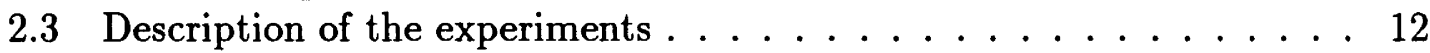

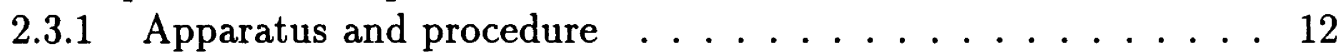

2.3.2 Flow visualization . . . . . . . . . . . . . . . 14

2.3.3 Definition and measurement of wave properties . . . . . . 14

2.4 Propagation of isolated waves $\ldots \ldots \ldots \ldots \ldots \ldots$

2.4 .1 Wave generation . . . . . . . . . . . 15

2.4.2 Experimental observations . . . . . . . . . . . . . . 17

2.4.3 Wavelength-amplitude scaling . . . . . . . . . . . . 19

2.4 .4 Wavespeed . . . . . . . . . . . . . . . 22

2.4.5 Amplitude attenuation . . . . . . . . . . . . . 22

2.5 Wave-boundary and wave-wave interactions $\ldots \ldots \ldots \ldots \ldots$

2.5.1 Reflection from a solid vertical boundary . . . . . . . . . 26

2.5.2 Head-on collision between two waves . . . . . . . . 28

2.6 Summary and conclusions $\ldots \ldots \ldots \ldots \ldots$

3 The coupling of waves and convection in rectangular channels 35

3.1 Introduction . . . . . . . . . . . . . . 35

3.2 Review of the literature $\ldots \ldots \ldots \ldots \ldots \ldots$

3.2 .1 Stability analysis . . . . . . . . . . . 36

3.2.2 Formation of a diffusive interface $\ldots \ldots \ldots \ldots \ldots$

3.2.3 Fluxes across a diffusive interface . . . . . . . . . . 39

3.3 Description of the experiments . . . . . . . . . . . 41

3.3 .1 Experimental setup . . . . . . . . . . . . . . 42

3.3.2 Independent parameters . . . . . . . . . . . . . 42

3.3.3 Apparatus and procedure . . . . . . . . . . . 43

3.3 .4 Flow visualization . . . . . . . . . . . . . . 43

3.3.5 Estimation of salt and sugar concentrations . . . . . . . 45

3.4 Experimental observations . . . . . . . . . . . . . . 46

3.4 .1 Filling disturbances . . . . . . . . . . . 46

3.4 .2 Transient behaviour . . . . . . . . . . . . 48

3.4.3 Long-term cyclic behaviour . . . . . . . . . . . . . 58

3.4.4 Run-down behaviour . . . . . . . . . . . . . . 64 


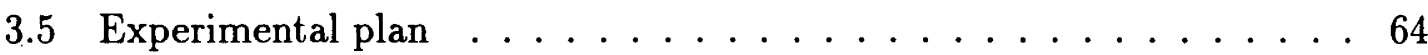

3.6 Results: wave-convection coupling . . . . . . . . . . . 66

3.6.1 Onset of wave-convection coupling . . . . . . . . . . . 66

3.6.2 Definitions and measurements . . . . . . . . . . 66

3.6.3 Wavespeed ........................ 68

3.6.4 Effects of the channel aspect ratio . . . . . . . . . 73

3.7 Results: interfacial fluxes . . . . . . . . . . . . . . 73

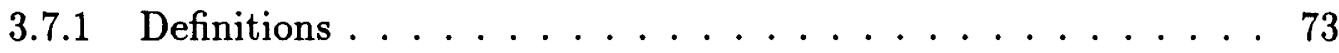

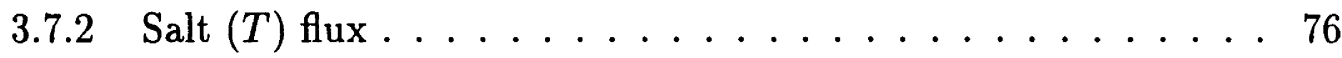

3.7.3 Flux ratio ...................... 78

3.7.4 Effects of wave-convection coupling on the fluxes . . . . . . . 78

3.8 Variations on a theme .................. 81

3.8.1 Variations in tank and cell geometries . . . . . . . . . . . 82

3.8.2 Variations in the double-diffusive system . . . . . . . . . . 82

3.8.3 Multiple diffusive interfaces . . . . . . . . . . . . . . 82

3.9 Summary and conclusions ................ 83

4 Coupled waves and convection in annular and three-dimensional cavities

4.1 Introduction . . . . . . . . . . . . . . . . 85

4.2 Experimental observations . . . . . . . . . . . . 86

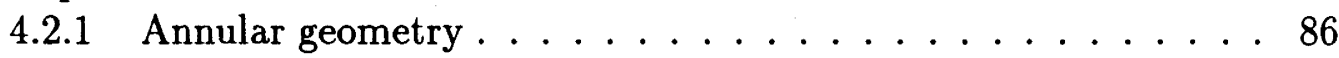

4.2 .2 Rectangular geometry .............. 88

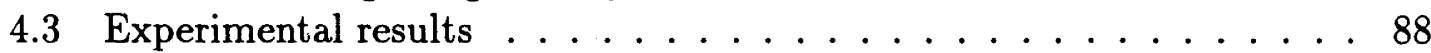

4.3.1 Onset of wave-convection coupling . . . . . . . . . . . 88

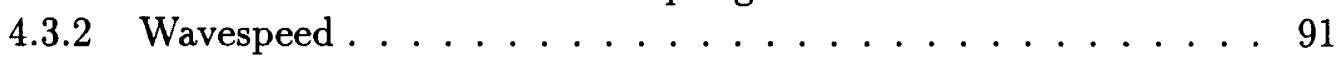

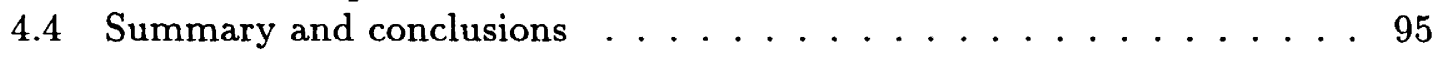

$\begin{array}{llr}5 & \text { Summary and conclusions } & 97\end{array}$

$\begin{array}{ll}\text { References } & 99\end{array}$ 


\section{List of Figures}

1.1 El Niño-Southern Oscillation $\ldots \ldots \ldots \ldots \ldots \ldots$

1.2 Atmospheric squall line . . . . . . . . . . . . . . 3

1.3 Travelling-wave convection in a binary fluid . . . . . . . 4

2.1 Experimental setup . . . . . . . . . . . . . . . 12

2.2 Experimental and theoretical density stratifications . . . . . . . 13

2.3 Definitions of amplitude and wavelength for mode-2 waves . . . . . 15

2.4 Photographs of wave generation . . . . . . . . . . . 17

2.5 Photographs of large- and small-amplitude waves $\ldots \ldots \ldots 18$

2.6 Wave asymmetry . . . . . . . . . . . . . 20

2.7 Wavelength $v s$. amplitude $\ldots \ldots \ldots \ldots \ldots \ldots \ldots \ldots \ldots$

2.8 Wavespeed results . . . . . . . . . . . . . . . 23

2.9 Amplitude attenuation results $\ldots \ldots \ldots \ldots \ldots \ldots$

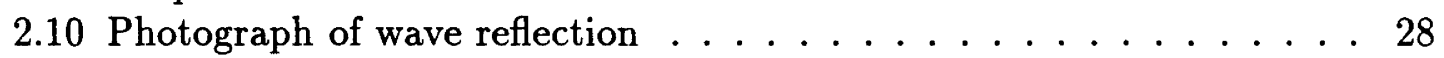

2.11 Phase shift results . . . . . . . . . . . . . . . . . . . 29

2.12 Photograph of collision between two waves of equal amplitude . . . 31

2.13 Photograph of collision between two waves of different amplitude. . . 33

3.1 Travelling-wave convection in a double-diffusive system . . . . . . 38

3.2 Structure of a diffusive interface . . . . . . . . . . . . 39

3.3 Standard experimental setup . . . . . . . . . . . . . . . . 44

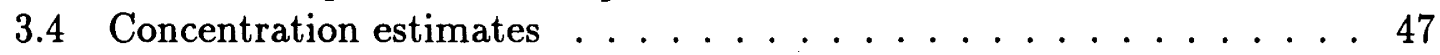

3.5 Illustration of flow after filling at high $R_{\rho} \ldots \ldots \ldots \ldots \ldots$

3.6 First transient flow regime $\ldots \ldots \ldots \ldots \ldots \ldots \ldots$

3.7 Second transient flow regime . . . . . . . . . . . . . 53

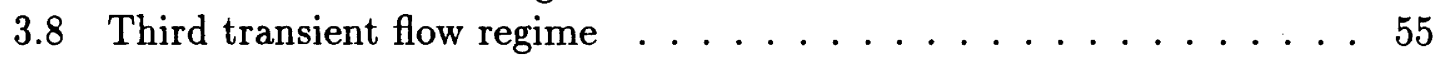

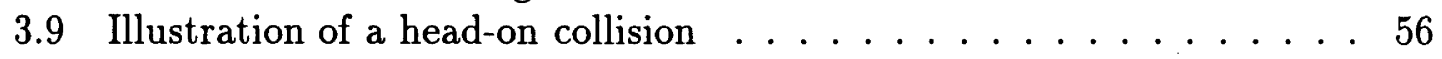

3.10 Illustration of an overtaking collision $\ldots \ldots \ldots \ldots$

3.11 Illustration of a quasi-steady cycle . . . . . . . . . . . 60

3.12 Direct photographs of a quasi-steady cycle . . . . . . . . . 61

3.13 Streak photographs of a quasi-steady cycle . . . . . . . . . 62

3.14 Photographs of the wave profile . . . . . . . . . . . . 64

$3.15 \bar{\alpha} \Delta T v s . R_{\rho}$ for the first set of experiments . . . . . . . . . . 67

3.16 Half-period of wavemotion vs. time since the start of the experiment 69

3.17 Wavespeed results for the first set of experiments . . . . . . . 71

3.18 Wave-convection coupling model . . . . . . . . . . . . . . 72

$3.19 \bar{\alpha} \Delta T v s . R_{\rho}$ for the second set of experiments . . . . . . . . . 74

3.20 Wavespeed results for the second set of experiments . . . . . . 75

3.21 Salt $(T)$ flux estimates . . . . . . . . . . . . . . 77

3.22 Salt $(T)$ flux results . . . . . . . . . . . . . . . 79

3.23 Flux ratio results . . . . . . . . . . . . . 80 
3.24 Illustration of the flow with multiple interfaces . . . . . . . . 83

4.1 Illustration of the flow in an annulus ........... 87

4.2 Illustration of the flow in a square tank . . . . . . . . . 89

4.3 Schematic plan view of a the flow in a $30 \times 10 \mathrm{~cm}$ rectangular tank . . 90

$4.4 \bar{\alpha} \Delta T$ vs. $R_{\rho}$ for the annulus experiments . . . . . . . . . . . . . 91

4.5 Evolution of experiment $2 a \ldots \ldots \ldots$. . . . . . . . . . . . . . . . . . . 92

4.6 Wavespeed results ......................... 94 


\title{
Chapter 1
}

\section{Introduction}

\author{
The kindly earth shall slumber, lapt in universal law \\ - Alfred, Lord Tennyson
}

Waves are a common form of fluid motion, and can convey energy and momentum over large distances in gravitationally stable regions. Another well-known and important form of fluid motion is convection, which overturns fluid and transports heat from hot to cold regions. Occasionally, these two very different processes couple together and reinforce each other to produce dramatic phenomena.

One such phenomenon is the 'El Niño-Southern Oscillation', which involves the coupling of waves in the equatorial ocean thermocline with atmospheric convective circulations in the southern hemisphere (Bjerknes, 1969). Figure 1.1(a) illustrates the prevailing conditions in the tropical Pacific. Increasing sea surface temperature to the west drives a single large-scale atmospheric convective circulation cell, which in turn helps maintain the sea surface temperature gradient. Cool dry air descends in the eastern Pacific, then gains heat and moisture as it flows westward over the increasingly warm ocean. On reaching the western Pacific the air is warm enough to rise. During ascent it cools to saturation, the moisture condenses out and the latent heat produced assists the convective updrafts. The two-dimensional cell is then closed with air returning east in the tropopause. However, this system is unstable. For example, a reduction in the sea surface temperature gradient weakens the westward winds, which in turn allows the warm water to flow eastward and further reduce the sea surface temperature gradient. About every 3 to 7 years such instabilities cause a switch to an El Niño state for about 18 to 24 months (figure $1.1 b$ ). During these events the sea surface temperature is greatest in the central Pacific and, as a result, the atmospheric circulation reverses direction in the west.

A second example is atmospheric squall lines. These long-lived mesoscale flows result from interactions between a cold dense pool of air collapsing into a moist environment, and convection driven by latent heating at the front of the cold pool. Figure 1.2 illustrates the accepted form of a mid-latitude squall line (Thorpe, Miller $\&$ Moncrieff 1982). The low level shear not only impedes the progress of the cold pool, but also steepens the front so that oncoming air is deflected upwards at a greater angle than would otherwise be the case. This displaced air cools to saturation, its moisture condenses out and the latent heat produced drives convective updrafts which rise to the tropopause. At mid-latitudes the upper level shear is sufficiently weak that the convective updrafts remain coherent and drive two largescale circulation cells, which evolve in response to the propagation of the cold pool. These cells assist the continued propagation of the cold pool. In addition, whatever 


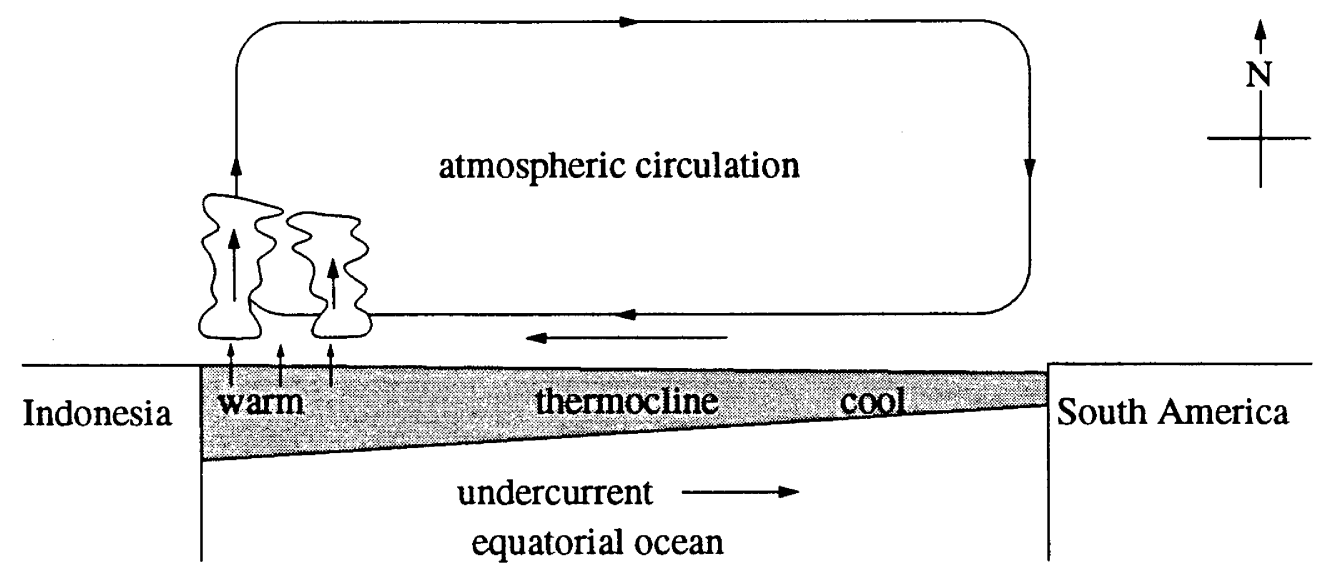

(a)

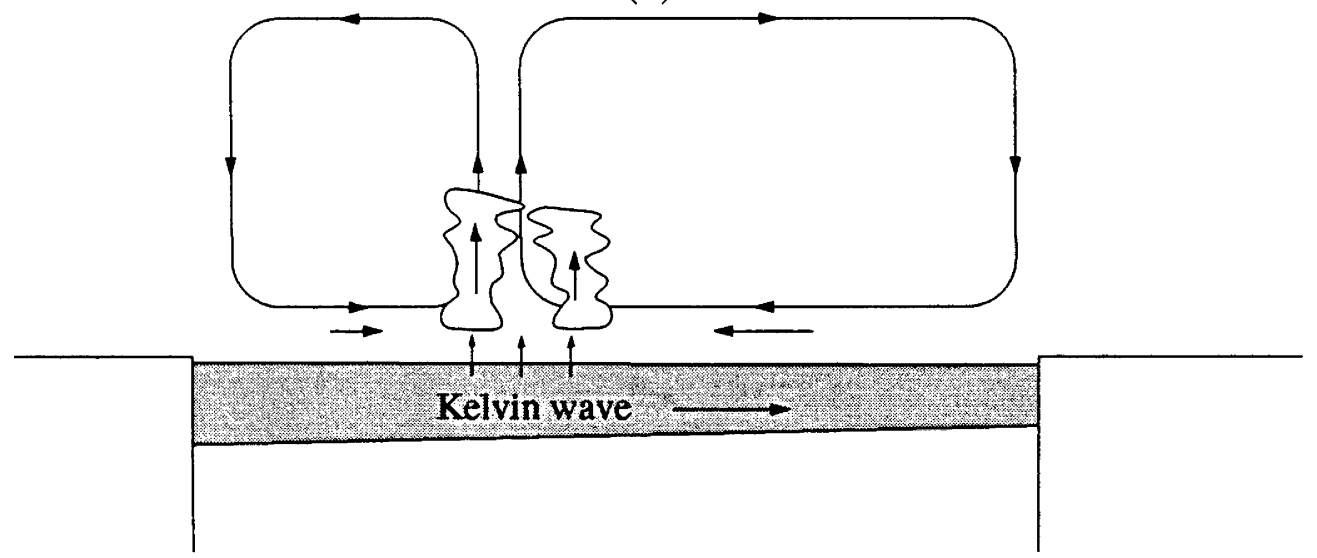

(b)

Figure 1.1: El Niño-Southern Oscillation. In the tropical Pacific momentum, heat and water vapour fluxes across the air-sea interface couple the ocean and atmosphere. (a) During the prevailing conditions the sea surface temperature increases to the west. This gradient drives a single atmospheric convective circulation cell, which in turn helps maintain the sea surface temperature. (b) Occasionally instabilities cause a switch to an El Niño state, during which the sea surface temperature is greatest in the central Pacific and the westward trade winds are disrupted. 


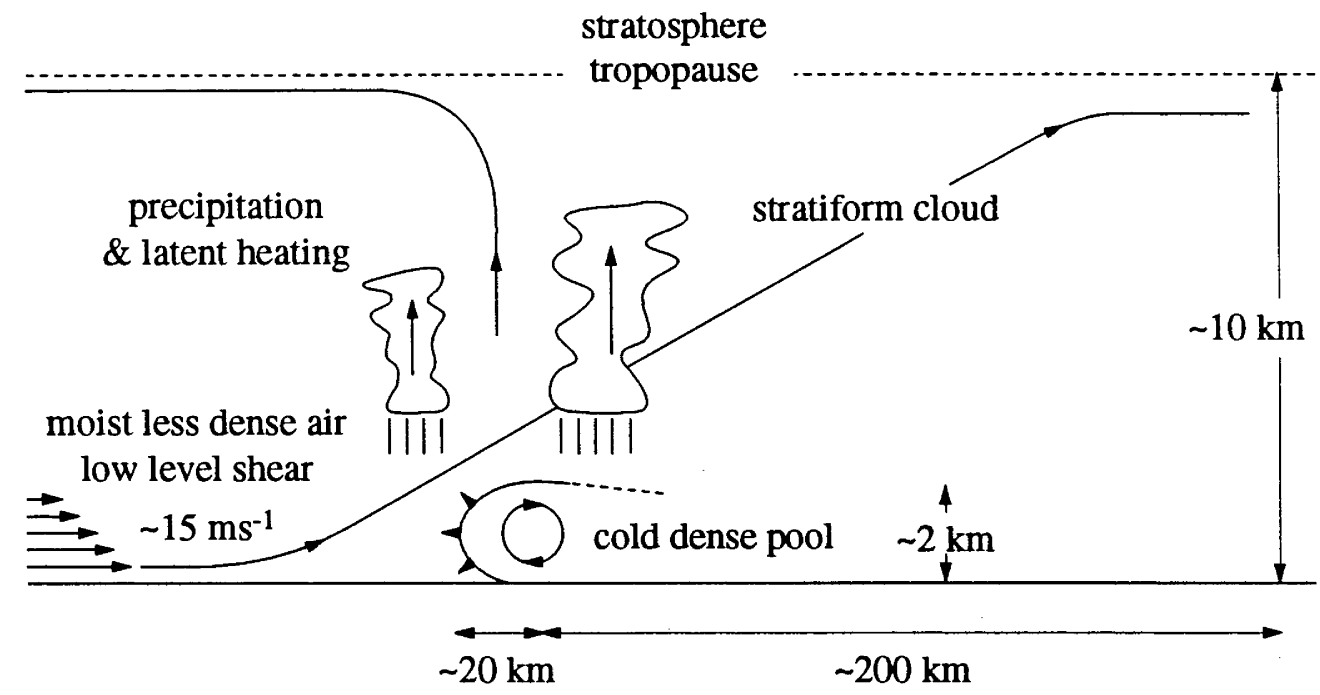

Figure 1.2: Atmospheric squall line. These mesoscale phenomenon involve the propagation of a cold dense pool of air into a moist environment. The low level shear not only impedes the progress of the cold pool, but also steepens the front so that oncoming air is deflected upwards. This displaced air cools to saturation, its moisture condenses out and the latent heat produced drives convective updrafts which give rise to two large-scale circulation cells. These cells evolve in response to the cold pool, while assisting its continued propagation. In addition, precipitation falls into the cold pool and the evaporative cooling maintains its negative buoyancy.

the balance between the convection and the ambient wind, the precipitation falls into the cold pool and the subsequent evaporative cooling maintains its negative buoyancy.

A non-geophysical example, which is nevertheless of fundamental importance, is the phenomenon known as 'travelling-wave' convection. This type of convection occurs for water-alcohol mixtures in which the diffusion of alcohol opposes convection via the Soret effect, i.e. the alcohol is less dense than water but diffuses towards colder regions. At onset the flow consist of cells that reverse direction periodically while remaining fixed in the horizontal. However, because alcohol diffuses more slowly than heat, this flow is unstable and the cells start to drift to the left or right (figure 1.3). The convective mixing destroys the stabilizing Soret-driven vertical alcohol gradient and well-mixed cells are driven by thin horizontal boundary layers at the top and bottom of the fluid layer. Alcohol from the boundary layers is fed into the plumes that separate cells, and it is the phase difference between the lateral concentration and temperature fields that causes the cells to travel (Walden et al., 1985).

This dissertation considers a closely related laboratory flow involving the "systematic interfacial wavemotion coupled to large-scale convection in the layers" which occurs when a layer of salt solution lies above a layer of sugar solution (Turner \& Chen, 1974). In this case the buoyancy flux through a 'diffusive' interface drives turbulent convection in both layers, which in turn spontaneously generates largeamplitude waves on the interface. The waves modulate the interfacial fluxes by modifying the interface thickness and thereby organize otherwise random convective motions into large-scale circulations. In turn, the waves themselves persist for unusually long times owing to energy input from the organized convection. Such flows provide an appropriate laboratory analog from which to develop a broader understanding of the processes by which waves and convection couple together. 


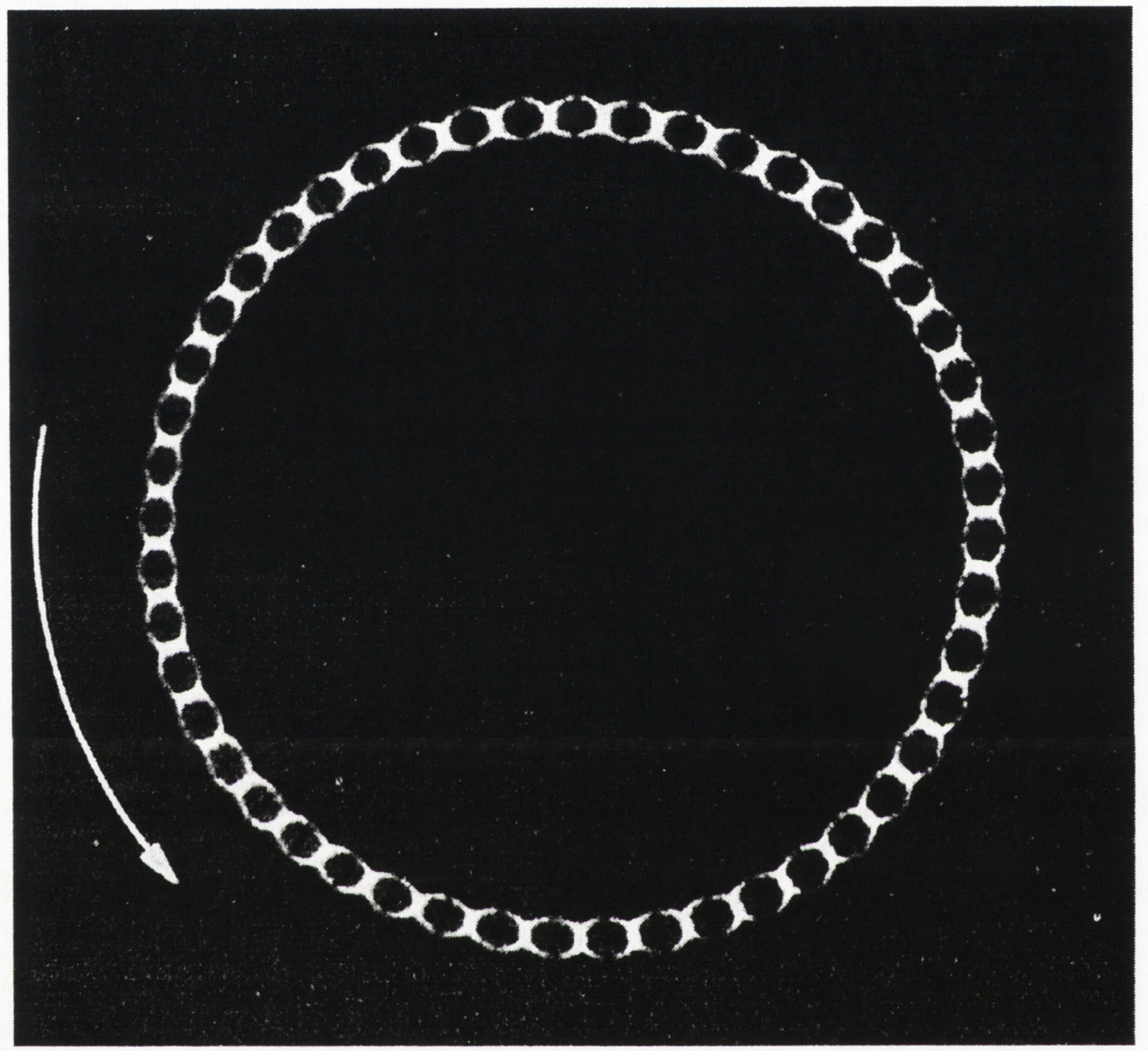

Figure 1.3: Travelling-wave convection in a binary fluid. A shadowgraph image of a convecting water-alcohol mixture in which the alcohol is less dense than water and diffuses towards colder regions. The first convective motions consist of cells that reverse direction periodically while remaining fixed in the horizontal. However, because alcohol diffuses more slowly than heat, this flow is unstable and the cells start to drift to the left or right. (From Bensimon et al. (1990).) 


\subsection{Research approach}

Before considering the waves propagating in phase with the ambient convection, it is first necessary to understand the behaviour of the same waves without convection. A relevant starting point is the vertical collapse of a thick mixed region of fluid along a thin interface separating two deep layers of constant density. According to Simpson (1982), the collapsing fluid takes the form of a gravity current intrusion for very thin interfaces and one or more internal solitary waves for slightly thicker interfaces. Whilst it is not clear a priori which, if either, of these flows is related to the waves propagating along a diffusive interface, the scaling and profile will be shown to be similar to 'deep-water' solitary waves (Benjamin, 1967; Davis \& Acrivos, 1967). Chapter 2 reports on detailed experimental measurements of such waves, focusing on those with large amplitudes and closed streamlines. Both the propagation of isolated waves and various wave-boundary and wave-wave interactions are discussed.

Chapters 3 and 4 return to the coupling of interfacial waves with convection. Preliminary experiments demonstrated that coupling occurs for a wide range of solutions including salt water above sugar water, cold fresh water above hot salt water and glycerol above potassium carbonate water. However, to simplify the investigation, analysis was restricted to two-dimensional flows with a single saltsugar interface separating two layers of equal depth. Chapter 3 considers flows in rectangular channels, for which a single wave propagated back-and-forth along the channel resulting in quasi-periodic reversals of the tank-scale convective circulation. Chapter 4 considers flows in an annulus, for which the waves and convection cells travelled in a continuous circular path without reflection.

Finally, in Chapter 5 the results pertaining to deep-water internal solitary waves and the coupling of such waves with convection driven from a diffusive interface are summarized. The latter flow is then compared to the El Niño-Southern Oscillation phenomenon and atmospheric squall lines. 


\title{
Chapter 2
}

\section{Deep-water internal solitary waves}

\author{
Order is heaven's first law \\ - An Essay on Man Epistle 4, Alexander Pope (1734)
}

\subsection{Introduction}

Solitary waves have been of interest since Russell (1837) first observed a surface water wave of finite amplitude and permanent form. This classical solitary wave propagates on the free surface of a shallow homogeneous fluid of constant depth. The permanent form is due to a balance between dispersion and nonlinearity. Linear theory predicts that infinitesimal shallow-water waves have phase speeds $c_{0}\left(1-\gamma k^{2}\right)$ where $k$ is the wavenumber, $\gamma$ is a constant and $c_{0}$ is the long wave $(k \ll 1)$ speed. Hence long waves travel fastest. Conversely, if dispersion is ignored then weakly nonlinear theory predicts phase speeds proportional to local displacement. Hence long waves travel slowest. Thus a balance between dispersion and nonlinearity is possible.

In recent years interest has centred on solitary waves propagating in density stratified fluids. These waves are important in both atmospheric physics and oceanography, with the Morning Glory phenomenon in northern Australia (Christie, Muirhead \& Hales 1978) and, possibly, Jupiter's Great Red Spot (Maxworthy \& Redekopp, 1976) being stunning examples. Indeed, Maxworthy (1980) noted that "[because] quite general and uncontrolled mixing events create internal solitary wave trains [this] leads us to suspect that they should be excited under many circumstances in natural systems".

This study considers long deep-water waves, that is, waves with wavelengths much smaller than the total fluid depth but much greater than the depth of the stratification. At small amplitudes such waves have open streamlines and are well described by weakly nonlinear theory (Benjamin, 1967; Davis \& Acrivos, 1967). However, at large amplitudes waves contain fluid trapped within closed streamlines and fully nonlinear theory is required. Tung, Chan \& Kubota (1982) computed numerical solutions for large steady waves in weakly stratified inviscid fluids but focused on shallow-water waves. Hence the theoretical and experimental basis on which to interpret field observations of large-amplitude deep-water waves is limited, and to redress this situation the present study made detailed experimental measurements of such waves. Laboratory experiments play an important role in the understanding of water waves because they allow time-dependence and dissipation to be investigated, both of which remain difficult to incorporate in numerical mod- 
els, and also provide the only information regarding wave-boundary and wave-wave interactions (Maxworthy, 1983).

In each experiment a single large-amplitude wave was generated by displacing a paddle into a diffused interface between two deep layers of constant density. The observations showed that fluid was entrained into, mixed and then ejected from the closed streamline region, and thus the wave not only transported fluid over large distances but also promoted localized mixing. As energy was dissipated the wave decreased in amplitude and eventually became too small to trap fluid. The measurements showed that the wavelength of large-amplitude waves increased linearly with increasing amplitude, and hence the wave profile only varied by a scaling factor, whereas weakly nonlinear theory shows that for small-amplitude waves to maintain permanent form the wavelength must be smaller for larger amplitudes. This selfscaling behaviour also occurs for deep-water waves on the interface between two immiscible fluids of constant density (Pullin \& Grimshaw, 1988) and thus is not a consequence of the presence of trapped fluid. However, despite the different scaling of small- and large-amplitude waves, the wavespeed increased linearly with increasing amplitude for all waves. The rate of amplitude attenuation was independent of amplitude, that is, the amplitude decreased linearly with position, and experimental observations indicated that dissipation occurred through viscous stresses, turbulent mixing and, possibly, wave-radiation. Attenuation was most rapid at small density differences and this trend was consistent with Reynold's number trends.

Experiments were also conducted to investigate wave-boundary and wave-wave interactions. During reflection from a solid vertical endwall all waves attained a maximum amplitude of about twice that of the incident wave, but otherwise there were qualitative differences between the behaviour of small- and large-amplitude waves. At small amplitudes there was no noticeable change in amplitude, whereas at large amplitudes the reflected wave was smaller and slower than the incident wave. This decrease in amplitude occurred because fluid was ejected from the largeamplitude waves through wave-breaking during the collapse away from the endwall. Furthermore, there was evidence that waves suffered a negative spatial phase shift. The head-on collision between waves of equal amplitude was similar to a reflection, with the fluid trapped within each wave reversing direction and being transported away from the collision along the path by which it approached. For waves with different amplitudes this behaviour was modified by the exchange of fluid from the larger incident wave to the smaller incident wave such that the leftward and rightward propagating waves had the same amplitudes before and after the collision. Thus, although the trapped fluid was principally reflected during head-on collisions, the waves themselves behaved like solitons in preserving their identity while they passed through each other.

The structure of this chapter is as follows. Section 2.2 reviews work on internal solitary waves and then $\S 2.3$ describes the experiments. This is followed in $\S 2.4$ by the presentation of observations and measurements of isolated waves. Section 2.5 then describes wave-boundary and wave-wave interactions.

\subsection{Review of the literature}

It is convenient to classify internal solitary waves as 'shallow-water' waves, 'deepwater' waves or 'finite-depth' waves depending on the relative magnitudes of the total fluid depth, the depth of the stratification and the wavelength. These classes of waves are briefly discussed and then a detailed review of deep-water waves is 
presented.

\subsubsection{Overview of internal solitary wave theory}

Theoretical work on internal solitary waves was initiated by Keulegan (1953) and Long (1956), who showed that such waves can propagate along the interface between two immiscible fluids of constant density. Following this, Benny (1966) and Benjamin (1966) derived the evolution equation governing solitary waves of smallbut-finite amplitudes, with wavelengths much greater than the total fluid depth, propagating in continuously stratified fluids where the depth of the stratification is similar to the total fluid depth. These waves are called shallow-water waves, for which the principal assumptions are

$$
\lambda / H \gg 1 \text { and } h / H=O(1),
$$

where $\lambda$ is the wavelength, $2 H$ is the total fluid depth and $2 h$ is the depth of the stratification. For a balance between dispersion and nonlinearity the wavelengthamplitude scaling is

$$
(\lambda / H)^{2}=O(a / H)^{-1}
$$

where $a$ is the amplitude.

The extension of shallow-water theory to account for waves propagating on a thin pycnocline in a fluid of infinite-depth was presented by Benjamin (1967) and Davis $\&$ Acrivos (1967). These waves are called deep-water waves and have wavelengths which are much smaller than the total fluid depth but much greater than the depth of the stratification:

$$
\lambda / H \rightarrow 0 \text { and } \lambda / h \gg 1 .
$$

For a balance between dispersion and nonlinearity the wavelength-amplitude scaling is

$$
\lambda / h=O(a / h)^{-1} .
$$

Kubota, Ko \& Dobbs (1978) extended the shallow- and deep-water theories to account for waves of small-but-finite amplitudes propagating in fluids which are neither shallow nor deep, but in which the total fluid depth is much greater than the depth of the stratification. These waves are called finite-depth waves and have wavelengths which are much greater than the depth of the stratification

$$
h / H \ll 1 \text { and } \lambda / h \gg 1 .
$$

Differences between these classes of waves are due to differences in dispersion. For shallow- and deep-water waves the dispersion relations for long waves are $c_{0}\left(1-\gamma k^{2}\right)$ and $c_{0}(1-\beta|k|)$ respectively, where $\beta$ is another constant. Comparing these expressions indicates that for shallow- and deep-water waves with identical $\lambda / H, \lambda / h$ ratios the deep-water waves must be more nonlinear in order to propagate with permanent form. The dispersion relation for finite-depth waves has a transcendental wavenumber dependence which reduces to the shallow- and deep-water dispersion relations in the respective limits.

\subsubsection{Deep-water internal solitary waves}

In contrast to the vast number of studies of shallow-water waves there have been few studies of deep-water waves. Theoretical studies have focused on the mathematically amenable problem of steady waves of small-but-finite amplitudes propagating in weakly stratified inviscid fluids. As computational power has increased 
time-dependent, arbitrary amplitude and dissipative flows have been investigated numerically. However, to illustrate the principal theoretical results, it is sufficient to consider steady waves.

Dubreil-Jacotin (1937) and Long (1953) showed that steady two-dimensional motions of an incompressible inviscid stratified fluid are governed by the conservation of vorticity equation

$$
\nabla^{2} \psi+\frac{1}{\rho} \frac{\partial \rho}{\partial \psi}\left[\frac{1}{2}\left(\psi_{x}^{2}+\psi_{z}^{2}\right)+g z\right]=\mathcal{H}_{\infty}(\psi)
$$

where $(x, z)$ are the horizontal and vertical coordinates, $\nabla^{2}=\partial^{2} / \partial x^{2}+\partial^{2} / \partial z^{2}$ is the Laplacian operator, $\psi(x, z)$ is the two dimensional streamfunction, $\rho(x, z)$ is the fluid density, $g$ is the gravitational acceleration and $\mathcal{H}_{\infty}(\psi)$ is a function determined by the upstream conditions. This equation can be used to consider the steady motions associated with a wave propagating at constant velocity $c$ through a stationary environment by employing a co-ordinate system which moves with the wave. In this reference frame there is a uniform flow upstream and $\mathcal{H}_{\infty}(\psi)$ is thus given by

$$
\mathcal{H}_{\infty}(\psi)=\frac{1}{\rho} \frac{\partial \rho}{\partial \psi}\left(\frac{c^{2}}{2}+\frac{g \psi}{c}\right)
$$

To simplify the analysis, and allow comparison with experiments, the following non-dimensional variables are introduced

$$
\tilde{x}=x / h, \quad \tilde{z}=z / h \quad \text { and } \quad \tilde{\psi}=\psi /(c h),
$$

and the stratification expressed as

$$
\rho(\psi)=\bar{\rho}[1-\sigma \mathcal{F}(\tilde{\psi})]
$$

where hereinafter - denotes a vertically averaged quantity, $\sigma$ is the non-dimensional density difference and $\mathcal{F}(\tilde{\psi})$ is an arbitrary monotonically increasing function. Furthermore, because it is deviations from the uniform flow that are of interest, the perturbation streamfunction $\tilde{\phi}(\tilde{x}, \tilde{z})$ is defined by

$$
\tilde{\psi}=\tilde{\phi}+\tilde{z}
$$

By substitution it can be shown that the governing equation may be rewritten as

$$
\nabla^{2} \tilde{\phi}+\Lambda\left(\frac{\mathcal{F}_{\psi}}{1-\sigma \mathcal{F}}\right) \tilde{\phi}=-\frac{1}{2}\left(\frac{\sigma \mathcal{F}_{\psi}}{1-\sigma \mathcal{F}}\right)\left(\tilde{\phi}_{\bar{x}}^{2}+\tilde{\phi}_{\bar{z}}^{2}+2 \tilde{\phi}_{\bar{z}}\right)
$$

where the subscripts indicate derivatives and

$$
\Lambda^{-1} \equiv c^{2} / \sigma g h=c^{2} / 2 c_{0}^{2}
$$

In general, the Boussinesq approximation, which neglects density variations in the inertial terms of the equations of motion, is applied and the Dubreil-JacotinLong equation reduces to

$$
\nabla^{2} \tilde{\phi}+\Lambda \mathcal{F}_{\psi}(\tilde{\phi}+\tilde{z}) \tilde{\phi}=0
$$

However, even for weakly stratified fluids $(\sigma \ll 1)$, this approximation must be treated with caution. For example, Long (1965) and Benjamin (1966) showed that 
errors occur for shallow-water waves in the weakly nonlinear limit, whereas Benjamin (1967) found that such errors do not occur for deep-water waves. In addition, for stratifications with a Brunt-Väisälä frequency which is symmetric about the centre of the fluid layer, $\mathcal{F}_{\psi}(\psi)=\mathcal{F}_{\psi}(-\psi)$, applying the Boussinesq approximation restricts the analysis to antisymmetric waves.

Davis \& Acrivos solved the Dubreil-Jacotin-Long equation for Boussinesq fluids (2.13) for waves of small-but-finite amplitudes by expanding the streamfunction and wavespeed in powers of the amplitude

$$
\tilde{\phi}=\left(\frac{a}{h}\right) \tilde{\phi}^{(1)}+\left(\frac{a}{h}\right)^{2} \tilde{\phi}^{(2)}+\ldots \quad \text { and } \Lambda=\Lambda^{(0)}+\left(\frac{a}{h}\right) \Lambda^{(1)}+\ldots
$$

After stretching the co-ordinates in accordance with deep-water scaling, they found solutions for the case of a thin constant gradient region separating two deep layers of constant density and a continuous stratification of the form

$$
\rho(z)=\bar{\rho}[1-(\Delta \rho / 2 \bar{\rho}) \tanh (z / h)] .
$$

For this latter, physically attainable, stratification the wavespeed increased with increasing amplitude

$$
c / c_{0}=1+0.3(a / h)+O(a / h)^{2} .
$$

This result was independently derived by Benjamin (1967), who also showed that waves have Lorentzian profiles

$$
\phi=a \lambda^{2} /\left(x^{2}+\lambda^{2}\right)
$$

and that for the $\tanh (z / h)$ stratification the exact wavelength-amplitude scaling is

$$
\lambda=5 / 2 a .
$$

In addition to finding the exact solution for steady waves, Benjamin derived the equation governing unsteady waves by modifying the dispersion term in the shallowwater Korteweg-de Vries equation to that appropriate for infinitesimal deep-water waves.

The existence of such waves was confirmed experimentally by Davis \& Acrivos, who showed that they form when a small quantity of neutrally buoyant fluid is injected into a slightly diffused density interface between two deep fluids of constant density. Two types of waves were observed. First, there are 'small-amplitude' waves characterized by open streamlines and behaviour similar to that predicted by the weakly nonlinear theory. These waves propagate with only gradual amplitude attenuation, reflect off a solid vertical boundary without apparent energy loss and two such waves travelling in opposite directions pass through each other unchanged. The fluid particle velocities are always less than the wave speed. Second, there are 'largeamplitude' waves characterized by a region of fluid trapped within closed streamlines and behaviour qualitatively different from that of the small-amplitude waves. These waves have fluid particle velocities approximately equal to the wavespeed and rapidly decay into small-amplitude waves. Subsequently, Maxworthy $(1980,1983)$ showed that a train of large amplitude waves can form from quite general forcing mechanisms when the appropriate environment conditions exist. The waves are ordered according to amplitude, with the large fast waves leading the small slow waves, and the closed streamline region of each consists of two counter-rotating cells.

To explain the behaviour at large amplitudes, Davis \& Acrivos computed numerical solutions to the Dubreil-Jacotin-Long equation for Boussinesq fluids (2.13) for 

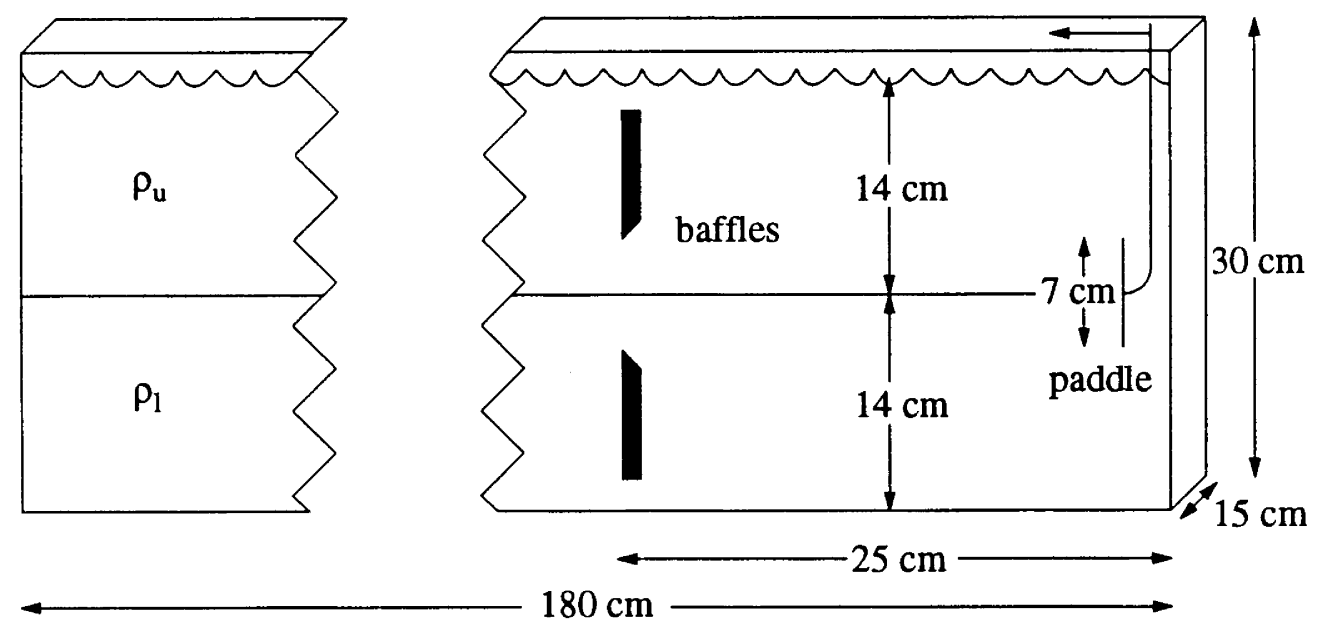

Figure 2.1: Experimental setup. Large-amplitude mode-2 waves were generated by smoothly displacing the paddle along the interface and then bringing it to rest between the two baffles. Some interfacial fluid became trapped against the moving paddle, and when the paddle was stopped this fluid slumped away under the restoring force of gravity and momentum imparted by the paddle. The front of this disturbance rapidly formed a large-amplitude solitary wave.

the case of a thin constant gradient region separating two deep layers of constant density. They found that for amplitudes $a / h>1.2$ the central streamline bifurcates trapping fluid within closed streamlines. Although the calculated wavespeeds agreed with experimental values, the analysis was invalidated by the presence of streamlines not connected to the upstream flow. Furthermore, the applicability of inviscid solutions is questionable because the diffusion of vorticity plays an important role in determining the dynamics of flows within closed streamline regions (Batchelor, 1956). However, Tung et al. (1982) noted that numerical solutions for large-amplitude waves resemble experimental observations, and argued that solutions are valid for times long enough that transient behaviour is negligible but short enough that viscosity is unimportant. On this premise they computed numerical solutions for large waves using the $\tanh (z / h)$ stratification. The results focused on shallow-water waves and indicated that once such waves develop a closed streamline region the wavelength starts to increase with increasing amplitude. In addition, the deep-water limit was shown to occur once the ratio of the total fluid depth to the depth of the stratification exceeds $H / h=10$.

\subsection{Description of the experiments}

\subsubsection{Apparatus and procedure}

The experiments were carried out in a glass channel of horizontal cross-section $L \times$ $w=180 \times 15 \mathrm{~cm}$ (figure 2.1). A continuously stratified fluid was formed by floating a layer of fresh water on top of a layer of salt water through two diffusers and then letting the interface produced thicken by diffusion. The salt water was easily adjusted to the desired density, and the layer depths were chosen to be as large as possible, $H=14 \mathrm{~cm}$, to ensure that the waves generated were deep-water waves. The filling procedure took about 20 minutes. Then the diffusers were removed, leaving a free upper surface, and the interface was allowed to thicken for a further 10 minutes. 


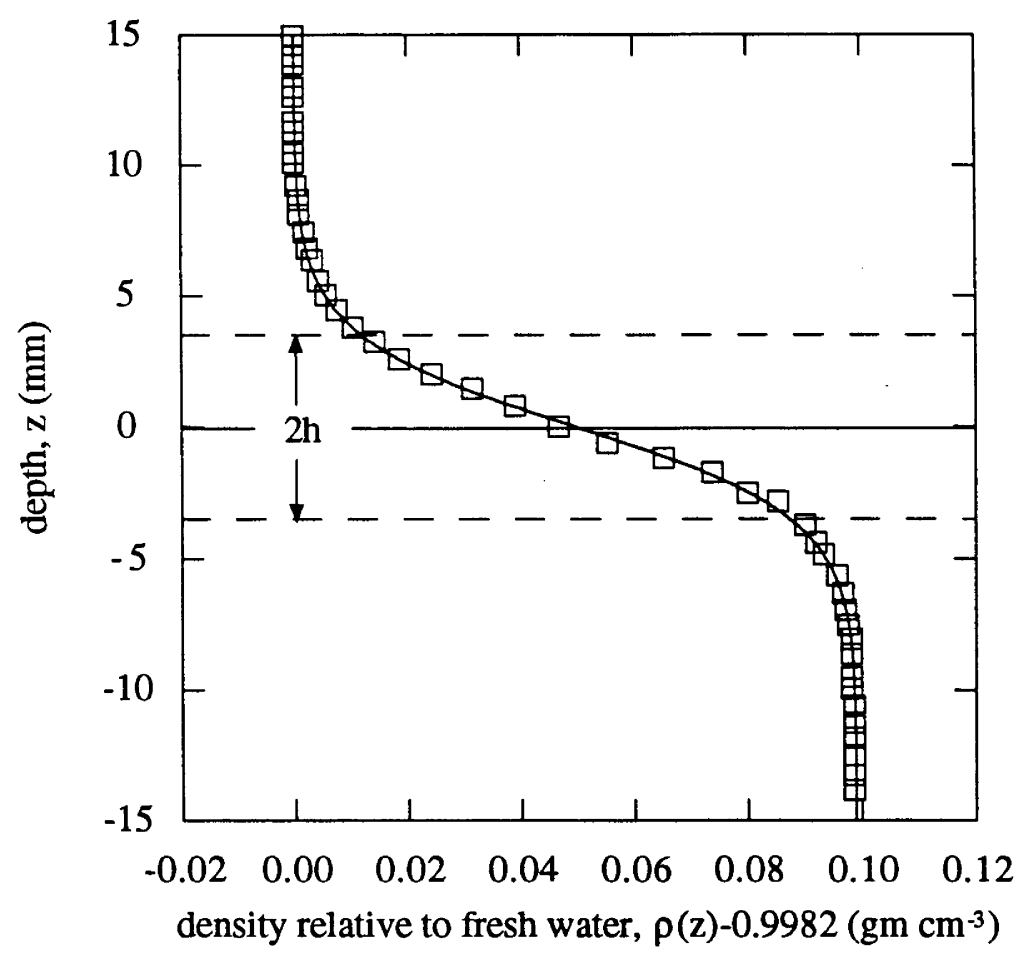

Figure 2.2: Experimental ( $\square$ ) and theoretical (—_ density stratifications. The experimental stratification was formed by allowing the interface between a fresh water layer to thicken by diffusion. Such stratifications were well described by the $\tanh (z / h)$ function used in theoretical work. In all experiments the ratio of the total fluid depth to the depth of the stratification was about $H / h=50$, which was large enough to ensure that the waves generated were deep-water waves.

At the end of this time the density stratification was examined by traversing a four wire conductivity probe (Precision Measurement Engineering, USA, Model MSCI-PME 106-300E) and fast response glass thermistor (Fenwal Electronics, USA, Model 0.070 inch diameter) through the interface. The measured conductivities were normalized to $20^{\circ} \mathrm{C}$ by assuming a $2.25 \%$ change per degree Celsius, and then Weast's (1975) data was used to calculate the densities at $20^{\circ} \mathrm{C}$. In all experiments the measured stratifications were well described by the $\tanh (z / h)$ function used in theoretical work, with a characteristic depth of about $2.5 \mathrm{~mm}$ (figure 2.2 ). Thus the ratio of the total fluid depth to the depth of the stratification was about $H / h=50$, which was well within the deep-water regime.

For a more accurate measure of the density difference across the interface $2 \mathrm{ml}$ samples were withdrawn from the centre of both layers. The density of samples was measured at $20.00 \pm 0.01^{\circ} \mathrm{C}$ using a digital densimeter (Anton Parr, K. G. Austria, Model DMA 02C) which gave a precision of $\pm 7 \times 10^{-5} \mathrm{gm} \mathrm{cm}^{-3}$.

The setup used to generate large-amplitude mode- 2 waves (i.e. lowest order varicose mode) is shown in figure 2.1. A paddle of depth $7 \mathrm{~cm}$ extended across the channel and was centred on the interface. This paddle was smoothly displaced along the interface and brought to rest between two polystyrene baffles. Some interfacial fluid became trapped against the moving paddle, and when the paddle was stopped this fluid slumped into the main chamber of the channel under the restoring force of gravity and momentum imparted by the paddle. The front of this intrusion quickly formed a large-amplitude solitary wave. Meanwhile, the baffles prevented the intermediate density fluid, created by mixing behind the paddle, from entering the main chamber of the channel and interacting with the wave. This generation 
technique was used because it consistently produced a single large-amplitude wave rather than several amplitude ordered waves. Furthermore, because the position and number of paddles was easily changed, this technique allowed wave-boundary and wave-wave interactions to be simply set up.

\subsubsection{Flow visualization}

Several flow visualization techniques were employed and, in addition to real-time observations, all experiments were recorded on video for subsequent analysis.

To determine the position, amplitude and wavelength of the waves, coloured water-insoluble droplets of different densities were injected into the fluid. Spread out along the channel, these droplets marked surfaces of constant density and thus, provided they were small compared to the length scale associated with shear, accurately marked the streamlines of the flow. Mixtures were made to within $\pm 0.0005 \mathrm{gm} \mathrm{cm}^{-3}$ of the desired densities from Heptane $\left(\rho=0.6840 \mathrm{gm} \mathrm{cm}^{-3}\right.$ at $\left.20^{\circ} \mathrm{C}\right)$, 1-Bromopentane $\left(\rho=1.2237 \mathrm{gm} \mathrm{cm}^{-3}\right.$ at $\left.20^{\circ} \mathrm{C}\right)$ and Sudan 3 dye. These chemicals were chosen for their insolubility in water, suitable densities and relative non-toxicity. Injecting the mixtures into the fluid with a fine gauge hypodermic needle placed below the free surface then produced droplets of $<0.25 \mathrm{~mm}$ diameter. In all experiments the surfaces of constant density corresponding to $z= \pm h$ in the $\tanh (z / h)$ profile were marked to allow the depth of the stratification and, as discussed in $\S 2.3 .3$, the wave properties to be measured.

Qualitative observations of the motion within the closed streamline region of large-amplitude waves were made by injecting dye, mixed with salt water so as to be neutrally buoyant, into the centre of the interface. This dyed fluid had similar properties to the undyed fluid. Usually dye was placed between the paddle and baffles so that it was entrained as the wave formed, but in some experiments dye was placed in the main chamber of the channel to investigate the possibility of entrainment into a fully formed wave. To complement these observations the centre of the interface was occasionally marked with droplets.

\subsubsection{Definition and measurement of wave properties}

Measurements of amplitude and wavelength were made in accordance with the definitions employed in weakly nonlinear theory (Benjamin, Davis \& Acrivos). Figure 2.3 illustrates these definitions and the general nature of the large-amplitude mode-2 waves observed.

The amplitude was defined as the maximum displacement of the $\psi= \pm h$ streamlines

$$
a=|\psi( \pm h)|_{\max }-h,
$$

and was measured using dyed water-insoluble droplets which were neutrally buoyant at $z= \pm h$.

The wavelength was defined as the half-width at the height where the amplitude, as defined by the $\psi= \pm h$ streamlines, was half its maximum value. This definition allowed for comparison with the weakly nonlinear theory of Benjamin and the numerical solutions of Tung et al. for large-amplitude waves. However, unlike the theoretical profiles, the large-amplitude waves generated in the laboratory did not exhibit fore-aft symmetry, that is, were not invariant under the transformation $x \rightarrow-x$. This asymmetry occurred because these waves mixed the density stratification and were followed by mode-1 (oscillatory) waves. Hence wavelength 


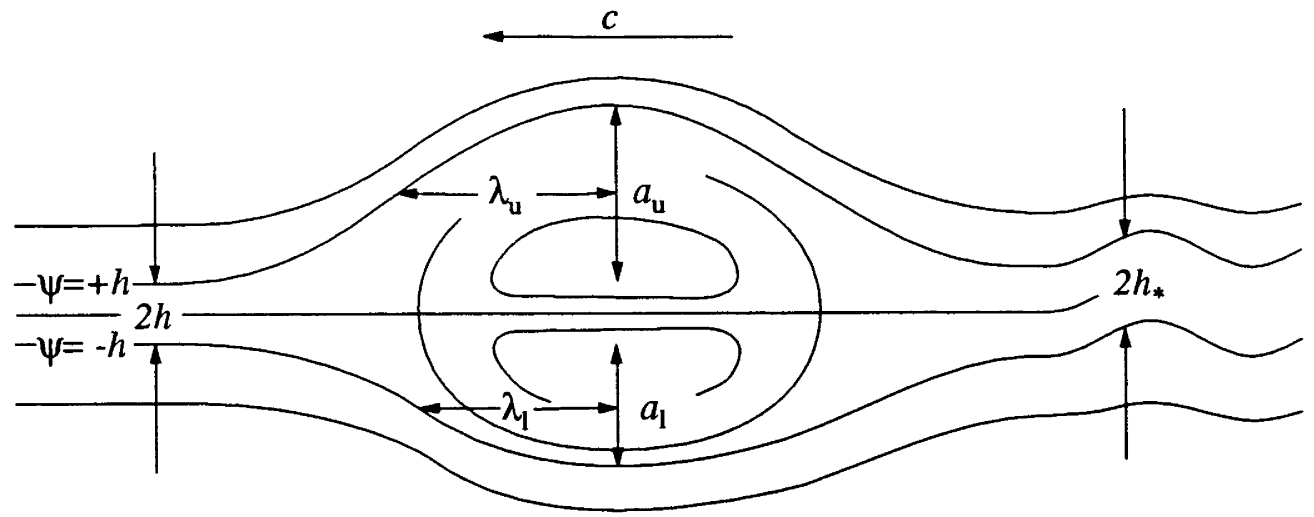

Figure 2.3: Definitions of amplitude and wavelength for mode-2 waves. The amplitude was defined as the maximum displacement of the $\psi= \pm h$ streamlines and the wavelength as the half-width at half-maximum of these streamlines. Large-amplitude waves mixed the density stratification (i.e. $h_{*}>h$ ) and were followed by mode-1 waves. Hence these waves did not exhibit fore-aft symmetry and, to facilitate comparison with the symmetric waves considered in theoretical work, wavelength measurements were restricted to the forward portion of waves and the depth of the undisturbed interface ahead of the wave used for non-dimensionalizations. Furthermore, to account for asymmetry about the centre of the interface, both the upper and lower values of the amplitude and wavelength were measured.

measurements were restricted to the forward portion of the wave and the depth of the undisturbed interface ahead of the wave was used for non-dimensionalizations.

Measurements of time, amplitude and wavelength were made every $5 \mathrm{~cm}$. The wave position was defined as the position of maximum displacement of the $\psi= \pm h$ streamlines, that is, the position of maximum amplitude. To account for asymmetry about the centre of the interface both the upper and lower values of the amplitude and wavelength were measured.

\subsection{Propagation of isolated waves}

A number of preliminary experiments were carried out for familiarization with the wave generation technique and wave properties. Once the experimental procedure was refined four experiments were carried out for which detailed observations and measurements were made of an isolated wave. In these experiments the lower layer densities were $\rho_{\mathrm{l}}=1.0501,1.1099,1.1500$ and $1.1997 \mathrm{gm} \mathrm{cm}^{-3}$, and the density of the fresh upper layer was $\rho_{\mathbf{u}}=0.9982 \mathrm{gm} \mathrm{cm}^{-3}$ in each case (table 2.1). Here the experimental results are presented and compared with theoretical predictions.

\subsubsection{Wave generation}

Isolated large-amplitude mode- 2 waves were easily generated using the paddle technique described in $\S 2.3 .1$. As the paddle moved along the interface the interfacial fluid was displaced from its level of neutral buoyancy and, while most fluid was deflected above and below the paddle, some fluid became trapped against the paddle. When the paddle was brought to rest this trapped fluid slumped away under the restoring force of gravity and momentum imparted by the paddle. Figure 2.4 shows the subsequent behaviour. The front of the intrusion took the form of a stable solitary wave, while the rear of the intrusion was highly unstable with fluid ejected 


$\begin{array}{ccccccccc}\rho_{\mathrm{l}}\left(\mathrm{gm} \mathrm{cm}^{-3}\right) & \text { symbol } & \sigma & h(\mathrm{~mm}) & h_{*}(\mathrm{~mm}) & \bar{a}_{0} / h & -\mathrm{d} \bar{a} / \mathrm{d} X & R e_{h} & R e_{w} \\ 1.0501 & \triangle & 0.0253 & 2.25 & 3.00 & 3.1 & 0.0037 & 36.2 & 2416 \\ 1.1099 & \square & 0.0485 & 2.75 & 3.25 & 2.5 & 0.0031 & 65.4 & 3567 \\ 1.1500 & \bigcirc & 0.0707 & 2.50 & 2.90 & 3.1 & 0.0029 & 65.5 & 3931 \\ 1.1997 & \times & 0.0917 & 2.50 & 3.00 & 2.9 & 0.0025 & 70.7 & 4243\end{array}$

Table 2.1: Experimental parameters and results for isolated waves. In each experiment the layer depths were both $H=14 \mathrm{~cm}$, and the density of the fresh upper layer was $\rho_{\mathbf{u}}=$ $0.9982 \mathrm{gm} \mathrm{cm}^{-3}$. The other parameters defining the density stratification were the lower layer density $\left(\rho_{1}\right)$, the non-dimensional density difference $(\sigma)$ and the characteristic depth before $(h)$ and after $\left(h_{*}\right)$ the passage of the wave. Note that by entraining, mixing and then ejecting fluid the large-amplitude waves promoted localized mixing which increased the thickness of the interface, i.e. $h_{*}>h$. Also presented is the initial non-dimensional amplitude $\left(\bar{a}_{0} / h\right)$, and the preliminary experiments indicated that these values were an artefact of the generation technique and interface characteristics rather than an inherent property of the waves. Finally, in each experiment the rate of amplitude attenuation $(-\mathrm{d} \bar{a} / \mathrm{d} X)$ was constant, that is, independent of amplitude. The most rapid attenuation occurred at small density differences and this behaviour was consistent with the trends in the Reynolds numbers characterizing viscous dissipation within the interface $\left(R e_{h}\right)$ and at the sidewalls $\left(R e_{w}\right)$.

backwards through wave-breaking. In this manner, the intrusion quickly attained a distinct length and an isolated solitary wave emerged about $20 \mathrm{~cm}$ from the point at which the paddle was stopped. The fluid which was not incorporated into the wave formed a gravity current intrusion which was rapidly left behind. Meanwhile, in response to the injection of fluid into the main chamber of the channel, fluid flowed over the upper baffle and back into the generation chamber until both chambers equilibrated to equal depths.

The largest waves were generated with an intermediate paddle speed, long paddle stroke and paddle which was deep compared to the characteristic depth of the interface. An appropriate paddle speed was determined by trial and error; at large speeds most of the fluid was deflected around the paddle, whereas at small speeds the trapped fluid collapsed away from the moving paddle. To allow for measurements over the largest possible distance, the paddle stroke was chosen to be as short as possible, $25 \mathrm{~cm}$, while still ensuring that the volume of trapped fluid was large. The paddle used was as large as possible, $7 \mathrm{~cm}$ deep, without being so large that the volume of fluid injected into the main chamber of the channel caused a disruptive return flow over the baffles. With this setup the initial dimensional amplitude of waves was consistently about $7.5 \mathrm{~mm}$ for interfaces of characteristic depths $2.0 \leq h \leq 5 \mathrm{~mm}$. Thus the non-dimensional amplitude of waves was principally determined by the depth of the interface, and maximum amplitude $(\bar{a} / h=3.75)$ an artefact of the generation technique and interface characteristics, rather than an inherent property of the waves. ${ }^{1}$

\footnotetext{
${ }^{1}$ Motivated by this study, Matthew Stocks (private communication) repeated several experiments and found stable waves of amplitude $\bar{a} / h=5$. These waves were generated with the described apparatus in a uniform channel by further reducing the depth of the interface. Similarly large waves were also generated on a thicker interface using a strongly convergent channel.
} 


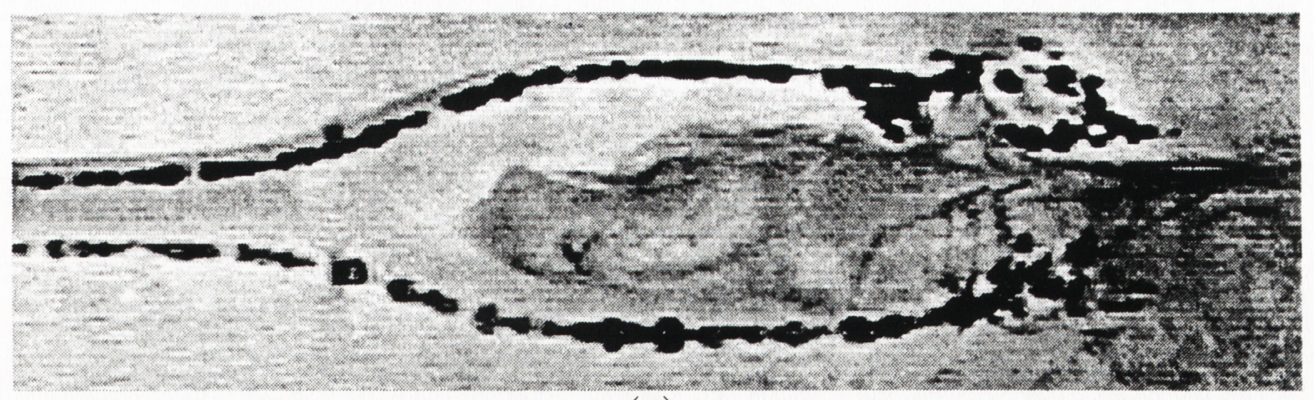

(a)

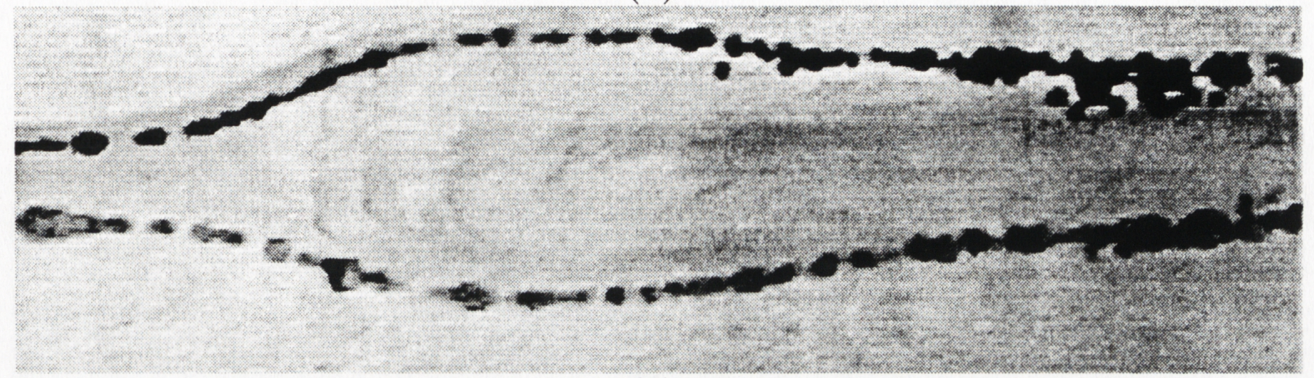

(b)

Figure 2.4: Wave generation. To visualize the flow surfaces of constant density corresponding to $z= \pm h$ in the $\tanh (z / h)$ profile were marked with dyed water-insoluble droplets. When the paddle was stopped the trapped fluid slumped away under the restoring force of gravity and momentum imparted by the paddle. (a) Initially the rear of the intrusion was highly unstable with fluid ejected backwards as a result of wave-breaking. (b) Seconds later the front of the intrusion had the form of a solitary wave.

\subsubsection{Experimental observations}

All waves decayed while propagating along the channel, and for large-amplitude waves this process was accompanied by a decrease in the size of the closed streamline region. In general, the waves traversed the channel twice before becoming too small to trap fluid, and then traversed the channel several more times before becoming too small to observe. Figure 2.5 shows the same wave at large and small amplitudes.

The large-amplitude waves had both laminar and turbulent regions. Along the open streamlines the flow was laminar and fluid stratified, whereas within the closed streamline region the flow was turbulent and fluid well-mixed. As observed in numerical solutions, the trapped fluid consisted of upper and lower counter-rotating cells. However, there was no clear boundary separating the cells, and fluid was continually entrained into and ejected from this 'closed' streamline region. For example, when a wave passed through a region of dyed interface the dye mixed into the cells within several seconds and was then gradually ejected from the rear of the wave in a laminar manner. The entrainment occurred at the same rate at all points along the boundary enclosing the trapped fluid, and the speed with which the dye spread through the cells was indicative of turbulent mixing. Furthermore, smaller droplets were occasionally entrained at the rear of the wave and then carried for $10-100 \mathrm{~cm}$ before being ejected from the rear of the wave in a turbulent manner. This behaviour was indicative of shear instabilities at the rear of the wave. Through this process of entraining, mixing and then ejecting fluid the large-amplitude waves promoted localized mixing which increased the thickness of the interface, i.e. $h_{*}>h$ (table 2.1).

A mode- 1 wave closely followed each mode- 2 solitary wave (figure 2.3 and yellow wave in figure 2.13 ). The amplitude of the mode- 1 waves was decreased by aligning 


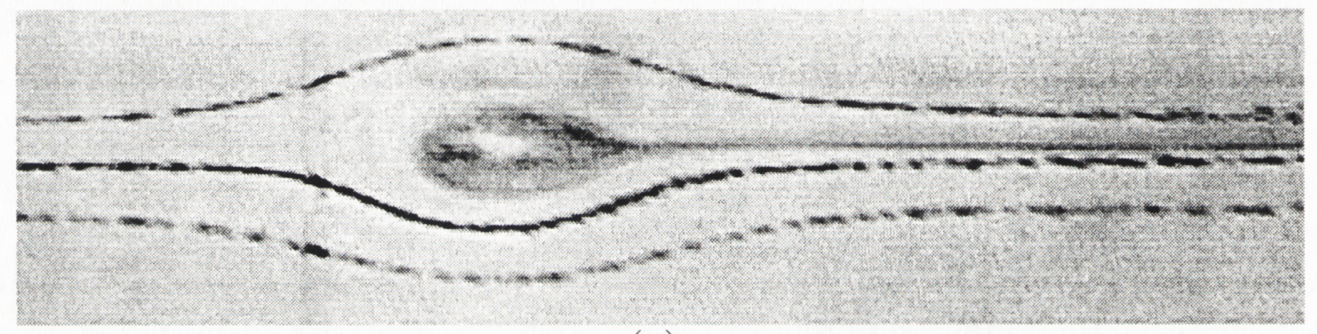

(a)

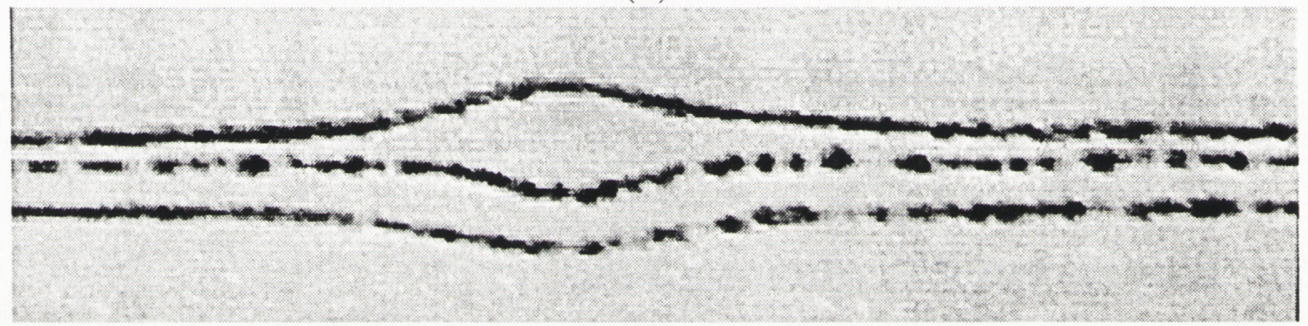

(b)

Figure 2.5: The same wave at large and small amplitudes. In both frames the wave is propagating to the left and, as evident from the displacement of the upper and lower droplets ( $\psi= \pm h$ streamlines), is asymmetric about the centre of the interface. (a) The wave has passed through a region of dyed interface and entrained dye marks the fluid trapped within closed streamlines. As observed in numerical solutions, the trapped fluid consisted of upper and lower counter-rotating cells, but in the experiments there was no clear boundary separating the cells and the motions were turbulent rather than laminar. The wave amplitude decreased and, as seen from the line of dye behind the wave, fluid was ejected from the cells. (b) Eventually the dye completely disappeared indicating that there was no longer any trapped fluid, and the subsequent laminar motions were well described by weakly nonlinear theory. 
the paddle with the centre of the interface and limiting the paddle speed, but these waves could not be eliminated. This behaviour was consistent with the prediction of Akylas \& Grimshaw (1992) that internal solitary waves of mode $n>1$ develop oscillatory tails. Of course, despite the care taken, it remains possible that the mode1 waves were an artefact of the generation technique and perchance propagated at similar speeds to the mode- 2 waves. To resolve this dilemma it is necessary to generate an isolated mode- 2 wave. In the future a more feasible, although not conclusive, test would be to compare the attenuation of mode- 2 waves with that expected if downstream wave-radiation were the dominant dissipation mechanism.

As seen in figure 2.5 , both large- and small-amplitude waves were asymmetric about the centre of the interface, i.e. $\phi(-z) \neq-\phi(z)$. Furthermore, the upper portion of the wave was occasionally slightly ahead or behind the lower portion of the wave. Subsequent analysis indicated that in each experiment the upper amplitude, $a_{\mathbf{u}}=|\psi(+h)|_{\max }-h$, was consistently greater than the lower amplitude, $a_{1}=|\psi(-h)|_{\max }-h$, whereas the wavelengths were similar (figure 2.6). This asymmetry could have resulted from non-Boussinesq effects, the salty lower layer being more viscous than the fresh upper layer, or the free upper and fixed lower boundaries. Indeed, the Dubreil-Jacotin-Long equation indicates that for density stratifications with a symmetric Brunt-Väisälä frequency, such as the $\tanh (z / h)$ stratification, Boussinesq fluids support antisymmetric waves, whereas non-Boussinesq fluids support asymmetric waves. However, it is likely that the observed asymmetry was primarily due to the influence of the upper and lower boundaries because the wave profile did not depend on the density difference as might be expected if nonBoussinesq or viscous effects dominated. In presenting further results the average amplitude, $\bar{a}$, and wavelength, $\bar{\lambda}$, values are employed.

Finally, all motions were two-dimensional. The waves were aligned straight across the channel, with sidewall boundary layers being too thin to observe, and there were no noticeable motions along the wave-front. This latter observation suggests that the large-amplitude waves were dissipated before the interaction between the counter-rotating cells and sidewalls could set up an Ekman boundary layer.

\subsubsection{Wavelength-amplitude scaling}

Solitary waves are characterized in terms of their wavelength-amplitude scaling, and this relationship provides a simple accurate basis for comparing experimental results with theoretical predictions.

In the experiments waves were observed to first decrease in amplitude and wavelength while propagating along the channel, however, once waves became too small to trap fluid, the amplitude attenuation was accompanied by an increase in the wavelength. Thus the scaling of small- and large-amplitude waves was qualitatively different; at small amplitudes the wavelength decreased with increasing amplitude, whereas at large amplitudes the wavelength increased with increasing amplitude. The behaviour at small amplitudes was consistent with the prediction of weakly nonlinear theory that for waves to maintain permanent form the wavelength must vary inversely with amplitude. Furthermore, the differences between experimental results and theoretical predictions at large amplitudes were not unexpected because the theory is only valid in the limit $a / h \rightarrow 0$.

Measurements of wavelength and amplitude were made throughout each experiment to complement the experimental observations. However, accurate measurements could not be made once the wave reflected from the endwall because the wave 


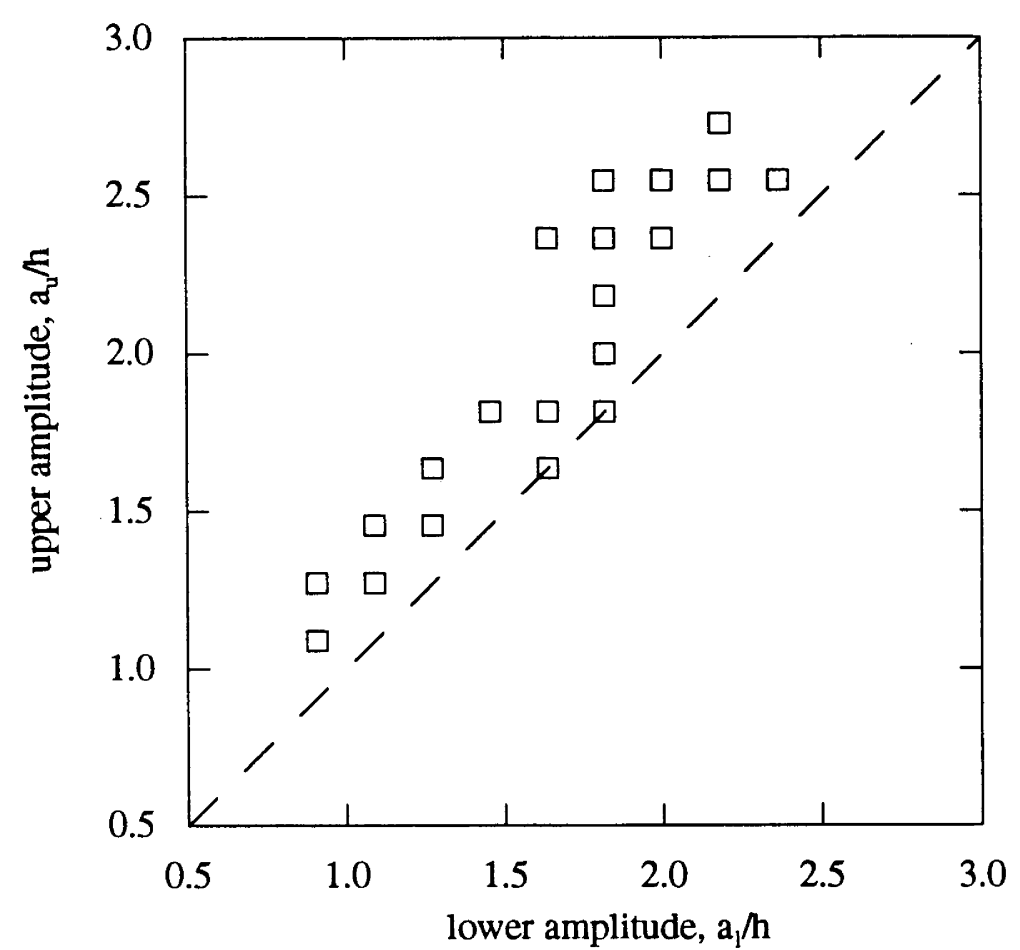

(a)

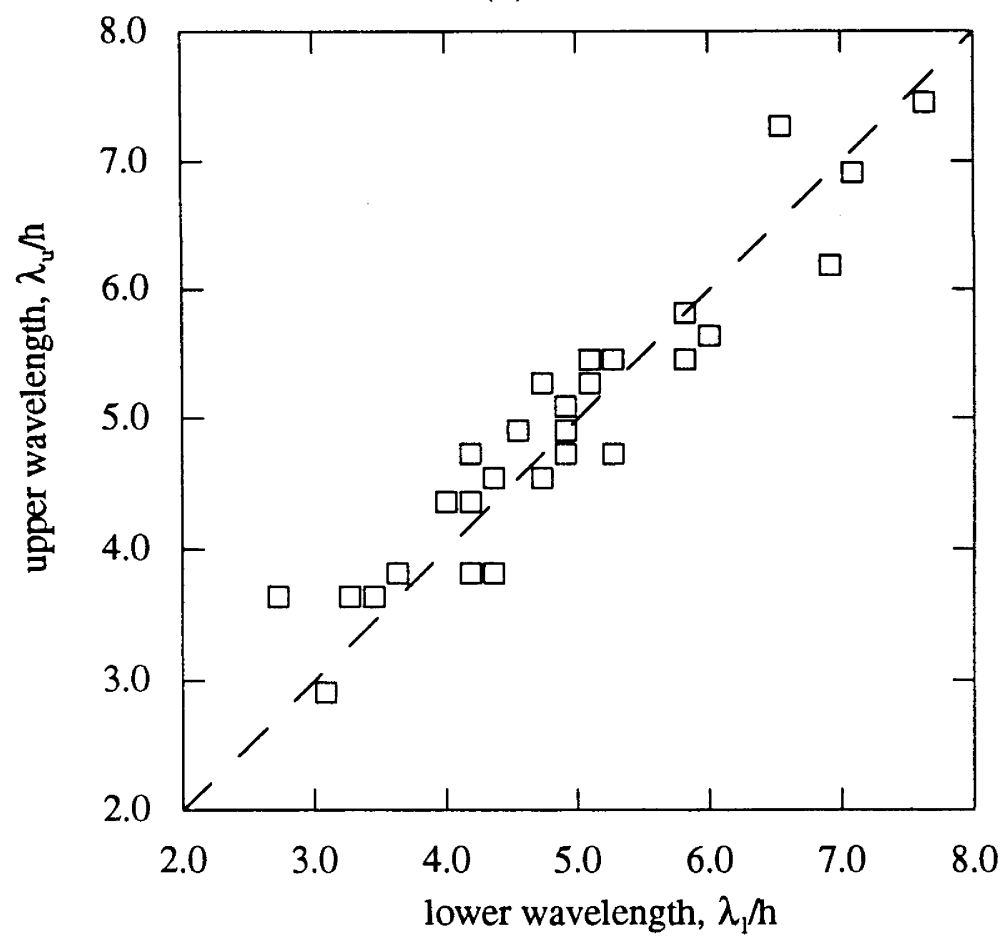

(b)

Figure 2.6: Wave asymmetry. Measurements of the upper and lower values of the (a) amplitude and $(b)$ wavelength as a wave traversed the channel. The dashed lines $\left(--{ }_{-}\right)$ denote values for an antisymmetric wave, i.e. $a_{\mathbf{u}}=a_{1}$ and $\lambda_{\mathbf{u}}=\lambda_{\mathbf{l}}$. In all experiments the upper amplitude was statistically greater than the lower amplitude, whereas the wavelengths were similar. This asymmetry was probably due to the influence of the free upper and fixed lower boundaries, but non-Boussinesq effects and the viscosity contrast between the two layers undoubtedly affected the wave profile. 


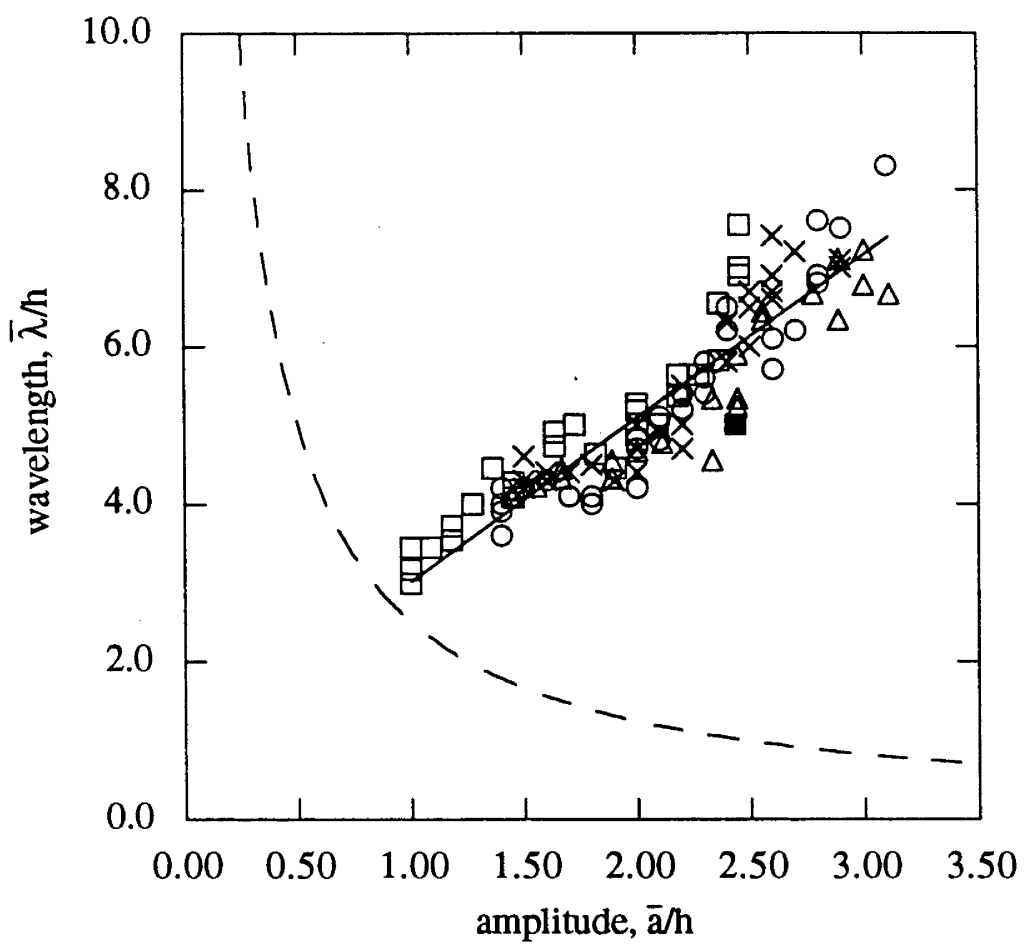

Figure 2.7: Wavelength vs. amplitude. In addition to the measured values, the inverse relationship derived by Benjamin using weakly nonlinear theory (- - - ) and a value inferred from the $H / h=40$ streamfunction plots calculated numerically by Tung et al. ( $\boldsymbol{\square})$ are plotted. The measurements indicate that at large amplitudes the wavelength increased linearly with increasing amplitude, with the solid line (- $\longrightarrow$ ) being the best linear fit for the entire dataset, and thus the wave profile only varied by a scale factor.

was distorted as it propagated back through the trailing oscillatory disturbance. (Indeed, even without this constraint, the validity of such measurements would be questionable because the profile of reflected waves was probably affected by the change in the structure of the density stratification resulting from the mixing by the incident wave.) Thus measurements were restricted to short times (large amplitudes) and it was not possible to determine the functional form of the wavelength-amplitude scaling relationship at small amplitudes.

The wavelength and amplitude values are plotted in figure 2.7 , and indicate that at large amplitudes the wavelength increased linearly with increasing amplitude

$$
\bar{\lambda} / h=0.95+2.1 \bar{a} / h \text { for } 1.0 \leq \bar{a} / h \leq 3.1 .
$$

A value inferred from the $H / h=40$ streamfunction plots of Tung et al. was consistent with the measurements, and this suggests that the wave behaviour can be accounted for by higher order amplitude terms without regard to viscous effects. Indeed, at large amplitudes the important dynamical balance must be between nonlinearity and dispersion, and thus viscosity may be important in dissipating energy but should not directly affect the wave scaling.

One consequence of this linear wavelength-amplitude relationship is that the waves were self-scaling, that is, the wave profile only varied by a scaling factor. This behaviour was first thought to occur because the volume of trapped fluid increased as the wave amplitude increased, but Pullin \& Grimshaw (1988) have found similar behaviour for deep-water waves on the interface between two immiscible fluids of constant density. Furthermore, the self-scaling behaviour provides a basis for similarity solutions and implies that the properties of all large-amplitude waves could 
be derived from a single numerical solution.

\subsubsection{Wavespeed}

Another important property of internal solitary waves is their speed of propagation. For each experiment the wavespeeds were calculated by taking the derivative of the third degree polynomial that best fits the displacement-time measurements, i.e. $\mathrm{d} X(t) / \mathrm{d} t$ where $X(t)=A_{0}+A_{1} t+A_{2} t^{2}+A_{3} t^{3}$. This procedure had two advantages over difference estimates. First, the video unit only recorded time to \pm 0.1 seconds and the curve fit smoothed the errors resulting from these quantized time measurements. Second, the derivation of an analytic expression for the wavespeed allowed values to be conveniently calculated at positions where the amplitude was known. The two wavespeed estimates agreed within expected uncertainties (figure $2.8 a$ ).

In most studies wavespeed results have been expressed in terms of the eigenvalue of the Dubreil-Jacotin-Long equation $\Lambda=2 c_{0}^{2} / c^{2}$. Although the relationship relating the wavespeed to the eigenvalue is simple, this approach has led to confusion in the interpretation of results, and thus here the wavespeeds themselves are presented.

Figure 2.8(b) shows the wavespeed plotted against the amplitude. To extend the range of amplitudes, the measurements presented by Davis \& Acrivos were included, and the wavespeed increased linearly with increasing amplitude according to

$$
c / c_{0}=1.0+0.49 \bar{a} / h \text { for } 0.1 \leq \bar{a} / h \leq 3.1
$$

for both large- and small-amplitudes. The weakly nonlinear theory was accurate at small amplitudes, but at large amplitudes the higher order amplitude terms were important and the measurements were underestimated. Accepting a $5 \%$ deviation between the theoretical and empirical curves suggests that the weakly nonlinear theory was valid for $\bar{a} / h<0.5$. In contrast the $H / h=40$ numerical solution presented by Tung et al. was in excellent agreement with measurements over the entire range of amplitudes, and this indicates that the Boussinesq approximation was valid even for density differences of $20 \%$. Finally, in view of the different wavelengthamplitude scaling of large- and small-amplitude waves, these results indicate that the wavespeed was an insensitive measure of wave behaviour.

\subsubsection{Amplitude attenuation}

All waves decayed while propagating along the channel, and for large-amplitude waves this process was accompanied by a decrease in the volume of trapped fluid. In general, the waves traversed the channel twice before becoming too small to contain fluid, and then traversed the channel several more times before becoming too small to observe.

To elucidate the attenuation process the wave amplitude was plotted against position in figure $2.9(a)$. In each experiment the amplitude decreased linearly with position, and thus the rate of attenuation was constant

$$
-\frac{\mathrm{d}(a / h)}{\mathrm{d}(X / h)}=-\frac{\mathrm{d} a}{\mathrm{~d} X}=\text { constant, }
$$

and calculated from the best linear fit to the measurements (table 2.1). The rates of attenuation are plotted in figure $2.9(b)$ and, even with the large errors, it is clear that attenuation was most rapid at small density differences. 


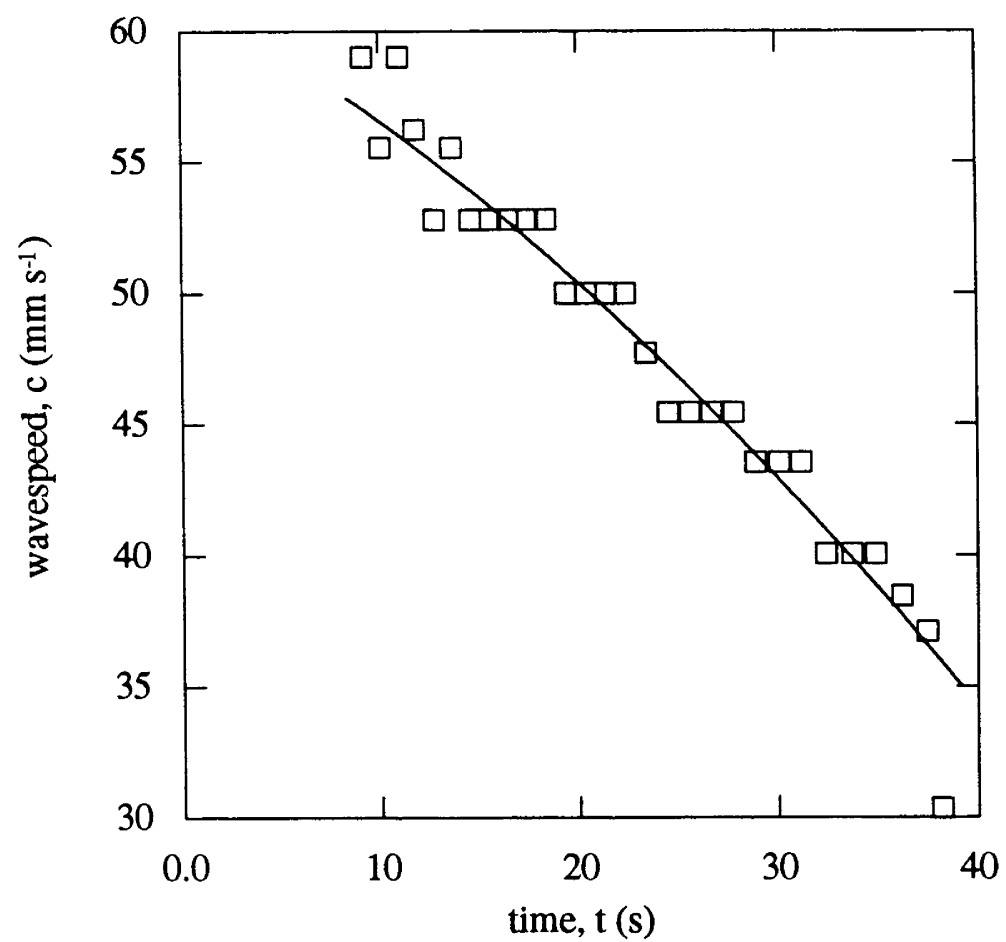

(a)

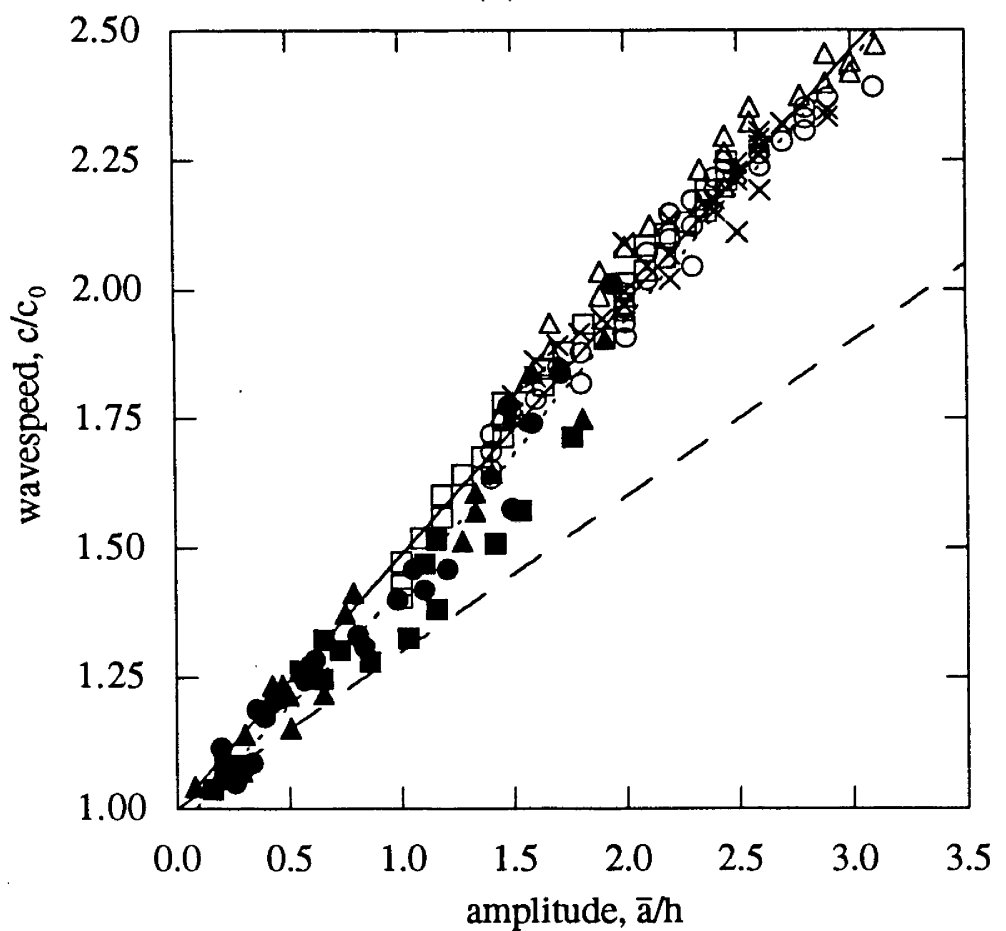

(b)

Figure 2.8: Wavespeed results. (a) Wavespeed estimates determined from a difference formula ( $\square$ ) and the derivative of the third degree polynomial that best fits the displacementtime measurements, $\mathrm{d} X(t) / \mathrm{d} t$ where $X(t)=A_{0}+A_{1} t+A_{2} t^{2}+A_{3} t^{3},(-\longrightarrow)$. The polynomial fit smoothed the errors resulting from quantized time measurements and allowed wavespeeds to be conveniently calculated at positions where the amplitude was known. (b) Wavespeed vs. amplitude. To extend the range of amplitudes, Davis \& Acrivos's measurements were included: $\rho_{1}=1.052 \mathrm{gm} \mathrm{cm}^{-3} \boldsymbol{\Lambda}, \rho_{1}=1.095 \boldsymbol{\square}$ and $\rho_{1}=1.095$. For comparison, the prediction of weakly nonlinear theory (- - -) and the $H / h=40 \mathrm{nu}-$ merical solution of Tung et al. $(\cdots \cdots)$ are also plotted. The wavespeed increased linearly with amplitude over the entire range of amplitudes, with the solid line ( - ) being the best linear fit, and was thus an insensitive measure of wave behaviour. 


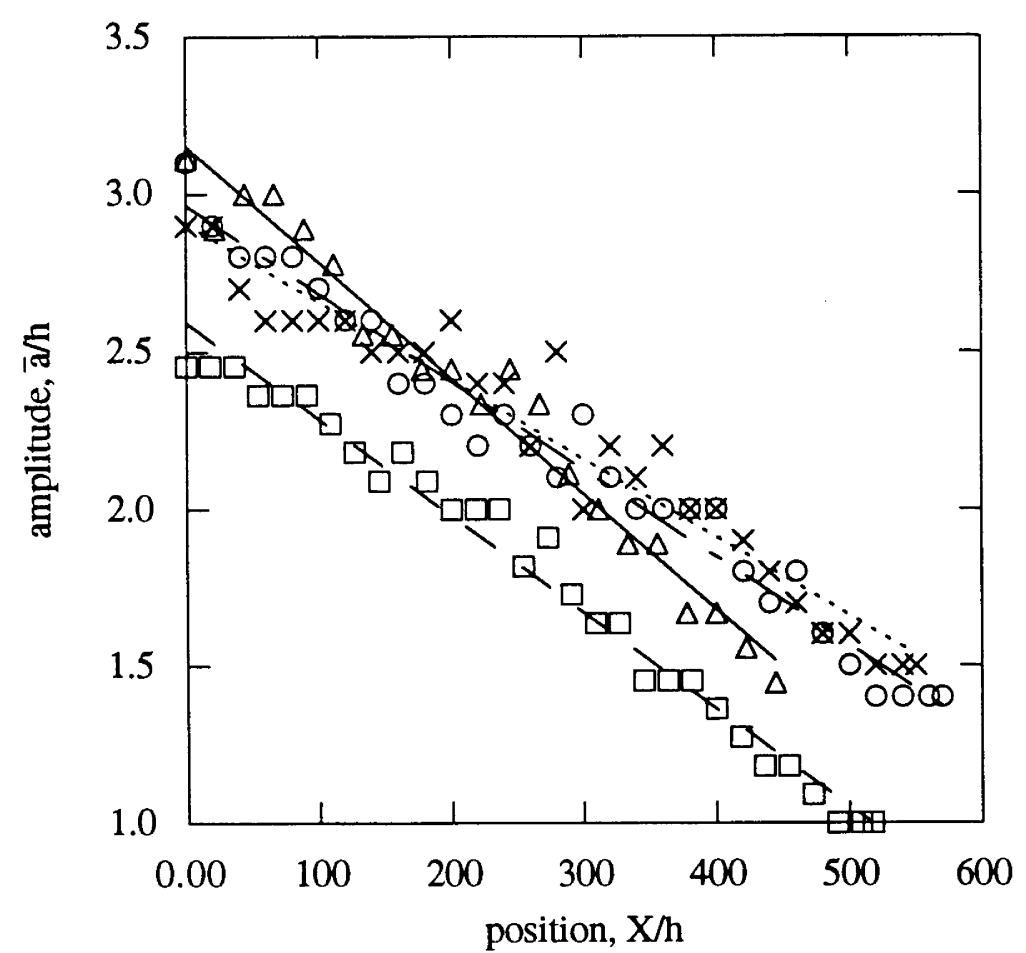

(a)

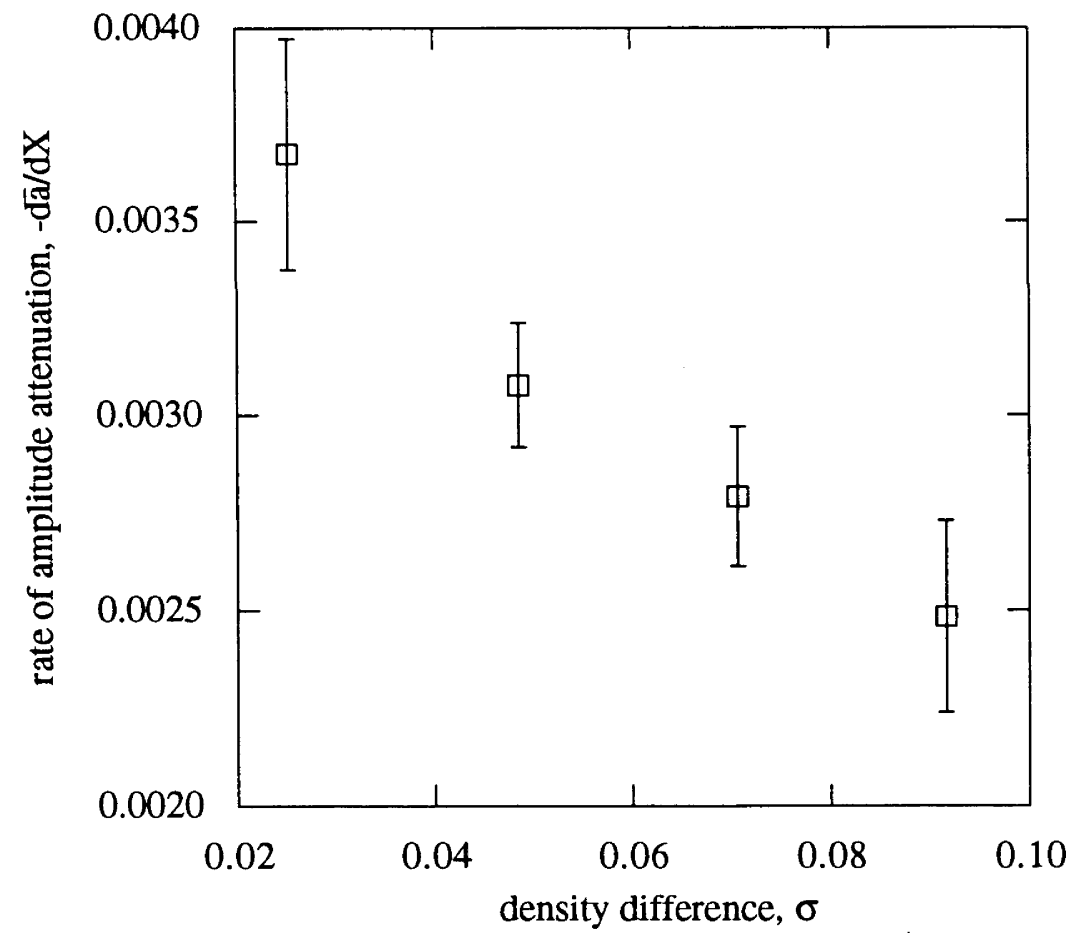

(b)

Figure 2.9: Amplitude attenuation results. (a) Amplitude vs. position. In each experiment the amplitude decreased linearly with position, and thus the rate of attenuation $(-\mathrm{d} a / \mathrm{d} X)$ was independent of the amplitude. The best linear fit is plotted for each experiment: $\rho_{\mathrm{l}}=1.05(-), \rho_{\mathrm{l}}=1.10(---), \rho_{\mathrm{l}}=1.15(--\longrightarrow)$ and $\rho_{\mathrm{l}}=1.20(\cdots \cdots)$. (b) Attenuation rate $v s$. density difference. The most rapid attenuation occurred at small density differences, and this trend was consistent with Reynold's number trends, whereas a Richardson number formulation of mixing predicted that the attenuation rate should be independent of the density difference. It was not possible to quantify the effects of turbulent mixing within the closed streamline region and downstream wave-radiation. 
A linear decrease of amplitude with position is not characteristic of most water waves. For example, both free surface waves (Lighthill, 1978) and solitary waves on an interface between two immiscible fluids (Koop \& Butler, 1981) attenuate more rapidly at large amplitudes than at small amplitudes, and thus exhibit an exponential decrease of amplitude with position. It is possible that similar behaviour occurs in the present situation, but was not observed because of the limited range of amplitudes considered. However, the experimental observations indicated that dissipation occurred through turbulent mixing and, possibly, wave-radiation as well as viscous stresses, and this difference probably accounts for the observed behaviour.

If viscous stresses were the dominant dissipation mechanism, then the rate of attenuation should depend on the Reynolds number; $R e=U L / \nu$ where $U$ is the velocity scale, $L$ is the length scale and $\nu$ the kinematic viscosity. Here attenuation was independent of amplitude and therefore the Reynolds numbers characterizing the flow should be independent of amplitude. Thus an appropriate velocity scale was the speed of long infinitesimal waves, and the length scales were taken to be the characteristic depth of the interface for dissipation within the interface

$$
R e_{h}=c_{0} h / \nu \propto\left(\sigma g h^{3}\right)^{\frac{1}{2}} / \nu(\bar{\rho}),
$$

and the channel width for dissipation at the sidewalls

$$
R e_{w}=c_{0} w / \nu \propto(\sigma g h)^{\frac{1}{2}} w / \nu(\bar{\rho}),
$$

Note that because salt water is more viscous than fresh water it was necessary to use the average viscosity of the two layers, $\nu(\bar{\rho})$, and the required values were calculated using Weast's data. Values of the Reynolds numbers are listed in table 2.1. It is seen that the change in the velocity scale caused by changes in the density difference dominated the associated changes in the viscosity, and thus both Reynolds numbers increased with increasing density difference. This result was consistent with the observation that the most rapid attenuation occurred at small density differences. Furthermore, in the preliminary experiments the rate of attenuation was observed to decrease when either the characteristic depth of the interface or channel width was increased. This behaviour was also consistent with the associated Reynolds number trends.

Large-amplitude waves were observed to entrain, mix and then eject fluid, and thus turbulent mixing occurred both within the closed streamline region as well as between this region and the surrounding flow. This latter process appeared to be the result of shear instabilities and should thus depend on the Richardson number $R i=g \Delta \rho L / \bar{\rho}(\Delta U)^{2}$. Again the velocity scale was taken to be the speed of long infinitesimal waves and the length scale as the depth of the stratification. This approach produced a constant Richardson number

$$
R i \propto g \Delta \rho 2 h / \bar{\rho} c_{0}^{2} \equiv g \Delta \rho h / \bar{\rho}(\sigma g h) \equiv \text { constant },
$$

that is, changes in the interfacial density difference led to changes in the stabilizing restoring force and destabilizing shear that were in balance. Therefore all waves should have had the same susceptibility to shear instabilities and, if this was the dominant dissipation mechanism, decayed at the same rate. However, the attenuation was observed to be most rapid at small density differences and thus this Richardson number approach was not appropriate.

As discussed in $\S 2.4 .2$, a mode- 1 wave followed each mode- 2 wave. It was not possible to determine whether these waves were an artefact of the generation 
technique or, as predicted by the Akylas \& Grimshaw, related to the main wave. However, if these waves were in fact radiated downstream from the main wave, then they could have carried significant amounts of energy away from the main wave. It was not possible to test this postulate. This was because attempts to correlate observations of the mode- 1 waves with the rate of attenuation of the mode- 2 waves were unsuccessful and attenuation laws for wave-radiation of trapped waves are not known.

In summary, evidence for a linear decrease of amplitude with position was convincing, and observations indicated that dissipation occurred through viscous stresses, turbulent mixing and, possibly, wave-radiation. Attenuation was most rapid at small density differences. This trend was consistent with Reynold's number trends, whereas a Richardson number formulation of the mixing between the trapped fluid and surrounding flow predicted that the attenuation rate should be independent of the density difference. It was not possible to quantify the effects of turbulent mixing within the closed streamline region and downstream wave-radiation.

\subsection{Wave-boundary and wave-wave interactions}

Since the discovery by Zabusky \& Kruskal (1965) that solitary waves described by the Korteweg-de Vries equation are stable and emerge from nonlinear interactions unchanged, there has been much interest in the types of interactions that can occur between solitary waves. Indeed, 'solitons' occur in many physical systems and show how coherent behaviour can form out of chaotic conditions (Krumhansl, 1991).

Interactions between solitary water waves are of special interest because experiments are simply set up and collisions between such waves are commonly observed in a variety of geophysical and technological situations. For example, to understand solitary Rossby waves in Jupiter's atmosphere, Maxworthy (1976) and Weidman \& Maxworthy (1978) investigated head-on and overtaking collisions between two solitary waves propagating on the free surface of a shallow layer of fluid. However, whilst the behaviour of shallow-water waves is known, the analogous behaviour of deep-water waves remains unknown. This lack of attention is particularly surprising given that such waves are easily generated and that collisions frequently occur between Morning Glory waves in northern Australia (Christie, 1992). Hence experiments were conducted to investigate the behaviour of deep-water waves during wave-boundary and wave-wave interactions.

The experiments were limited to the reflection of waves from a solid vertical boundary and the head-on collision of two waves. These latter experiments were set up using two paddles. Attempts to set up overtaking collisions were unsuccessful because, although a slow (small) leading wave and fast (large) following wave could be generated by stopping and then restarting the paddle, the channel was not long enough for the waves to collide before reaching the endwall. In all experiments the density of the lower layer was $\rho_{\mathrm{l}}=1.10 \mathrm{gm} \mathrm{cm}^{-3}$ and the density of the fresh upper layer was $\rho_{\mathbf{u}}=0.9982 \mathrm{gm} \mathrm{cm}^{-3}$.

\subsubsection{Reflection from a solid vertical boundary}

Three experiments were conducted to investigate the behaviour of waves during reflection from a solid vertical boundary, i.e. the channel endwall. The paddle location was varied so that waves propagated $X=80,120$ and $155 \mathrm{~cm}$ before reaching the endwall and amplitude attenuation ensured that the waves had different amplitudes 


$\begin{array}{ccccccccc}X(\mathrm{~cm}) & h_{\mathrm{i}}(\mathrm{mm}) & h_{\mathrm{r}}(\mathrm{mm}) & (\bar{a} / h)_{\mathrm{i}} & (\bar{a} / h)_{\mathrm{r}} & \left(c / c_{0}\right)_{\mathrm{i}} & \left(c / c_{0}\right)_{\mathbf{r}} & -\Delta X(\mathrm{~mm}) & \Delta t(\mathrm{~s}) \\ 80 & 2.25 & 2.50 & 2.2 & 1.9 & 2.2 & 1.7 & 7 & 0.3 \\ 120 & 2.25 & 2.50 & 2.0 & 1.7 & 2.0 & 1.5 & 3 & 0.2 \\ 155 & 2.75 & 3.25 & 1.0 & 0.8 & 1.5 & 1.2 & 4 & 0.4\end{array}$

Table 2.2: Experimental parameters and results for reflected waves. In each experiment the layer depths were both $H=14 \mathrm{~cm}$, the density of the lower layer was $\rho_{\mathrm{l}}=1.1000 \mathrm{gm} \mathrm{cm}^{-3}$ and the density of the fresh upper layer was $\rho_{\mathbf{u}}=0.9982 \mathrm{gm} \mathrm{cm}^{-3}$. Analysis of experiments was complicated because the localized mixing promoted by the incident wave meant that the reflected wave experienced different conditions, i.e. $h_{\mathrm{r}}>h_{\mathrm{i}}$ where the subscripts i and $\mathrm{r}$ refer to the incident and reflected waves. However, despite these complications, the direct amplitude measurements $(\bar{a} / h)$ and wavespeed measurements $\left(c / c_{0}\right)$ both indicated that the large-amplitude waves decreased in amplitude during reflection. Finally, the temporal $(\Delta t)$ and spatial $(-\Delta X)$ phase shifts inferred from the wave trajectories are also presented, and, despite the large errors associated with these quantities, the evidence suggesting that waves suffered a negative spatial phase shift is convincing.

at the endwall (table 2.2). In each experiment the incident wave was large enough to contain fluid at the first reflection, and thus behaviour at small amplitudes had to be obtained from subsequent reflections.

Several complications were encountered during the experiments. First, although it was simple to make measurements of incident waves, it was often impossible to make similar measurements of reflected waves because the trailing oscillatory disturbance distorted these waves. Second, the incident waves mixed the density stratification and thus the reflected waves experienced different conditions. Indeed, the inherent profile of reflected waves was probably affected by the change in the structure of the stratification, and the increase in thickness of the interface meant that if a wave was to maintain its non-dimensional amplitude the reflected wave actually had to be larger and faster than the incident wave. However, the amplitude and wavespeed measurements were consistent with each other, suggesting that the measurements were a useful addition to the qualitative observations.

Whilst all waves attained a maximum (dimensional) amplitude during reflection of about twice that of the incident wave, there were qualitative differences between the behaviour of small- and large-amplitude waves. At small amplitudes there was no noticeable change in amplitude, whereas at large amplitudes the reflected wave was smaller, and thus slower, than the incident wave (table 2.2). This decrease in amplitude occurred because fluid was ejected from the large-amplitude waves through wave-breaking during the collapse away from the endwall (figure 2.10). Such behaviour was similar to that during wave generation, and again the fluid not incorporated into the wave formed a gravity current intrusion which was rapidly left behind. It was not possible to determine whether the behaviour was sensitive to the amplitude of the incident wave, however the $X=155 \mathrm{~cm}$ wave suffered a much larger decrease in amplitude than the other waves. This anomalous behaviour probably occurred because on reaching the endwall this wave was only just large enough to contain fluid and it ejected all of this fluid during the reflection.

Figure 2.11(a) shows the trajectory followed by the $X=80 \mathrm{~cm}$ wave during reflection. As shown, extrapolating the lines fitted to the incident and reflected 


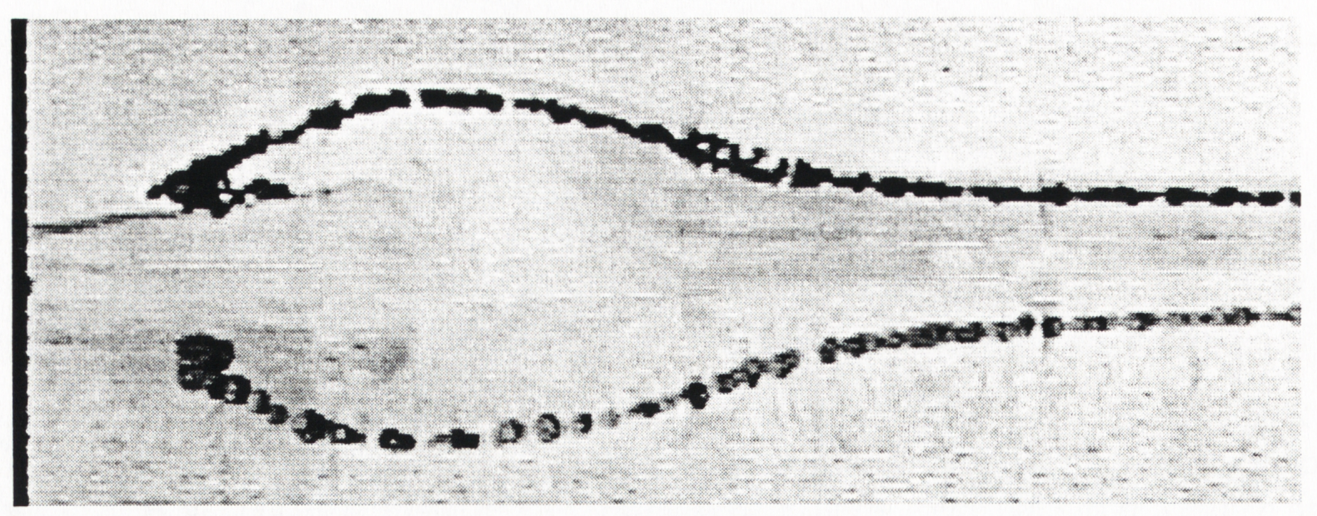

Figure 2.10: Wave reflection. The $X=80 \mathrm{~cm}$ wave during reflection from the endwall. Small waves reflected smoothly without a change in amplitude, whereas large waves ejected fluid through wave-breaking while collapsing away from the endwall and thus the reflected wave was smaller and slower than the incident wave.

trajectories indicates that the wave suffered a small negative spatial phase shift, that is, appeared to reflect from a virtual endwall behind the real endwall or, alternatively, appeared to hesitate at the endwall. Similar behaviour occurred in the $X=120$ and $155 \mathrm{~cm}$ experiments. Estimates of the spatial $(\Delta X)$ and temporal $(\Delta t)$ phase shifts are listed in table 2.2, and were left as dimensional quantities because non-dimensionalization was confused by the fact that the increase in the interface thickness during the passage of the incident wave resulted in different length and time scales being appropriate to the incident and reflected waves. Figure 2.11(b) shows the spatial phase shift plotted against (incident) wave amplitude. The point estimates indicate that all waves suffered similar phase shifts, but large errors produced by the extrapolation procedure hampered the analysis. However, despite these errors, evidence for phase shifts is convincing, with a statistical average of point estimates giving a non-zero value and this behaviour is similar to that for solitary waves propagating on the free surface of a shallow layer of fluid (Maxworthy, 1976).

\subsubsection{Head-on collision between two waves}

Several experiments were conducted to investigate the behaviour during head-on collisions between two waves propagating in opposite directions. Collisions between waves of equal amplitude were set up by displacing two paddles at the same time, whereas collisions between waves of different amplitudes were set up by displacing two paddles at different times. The distance that the waves propagated before colliding was varied but the channel was too short for a large range of amplitudes to be examined. Another problem was that, with a single video camera, it was difficult to make detailed measurements of both waves.

Figure 2.12 shows the collision between large-amplitude waves of equal amplitude. The behaviour was similar to a reflection from a solid vertical boundary, that is, the fluid trapped within each wave reversed direction and was transported away from the collision along the path by which it approached. Some fluid was ejected through wave-breaking as the waves collapsed away from each other resulting in both outgoing waves being smaller, and thus slower, than the ingoing waves. After reflecting off the paddle apparatus the two waves returned to the point of the original collision and, in agreement with the direct measurements, this indicated that both outgoing waves had the same amplitude. The fluid not incorporated into the 


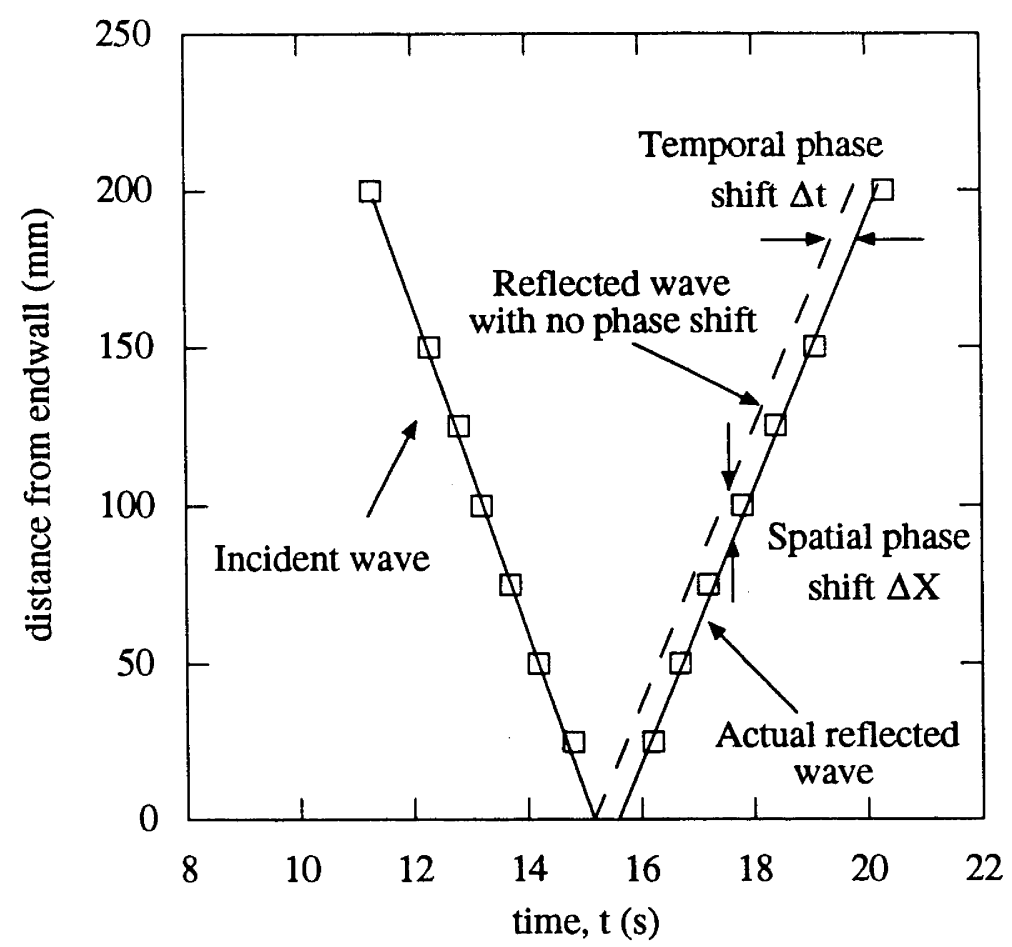

(a)

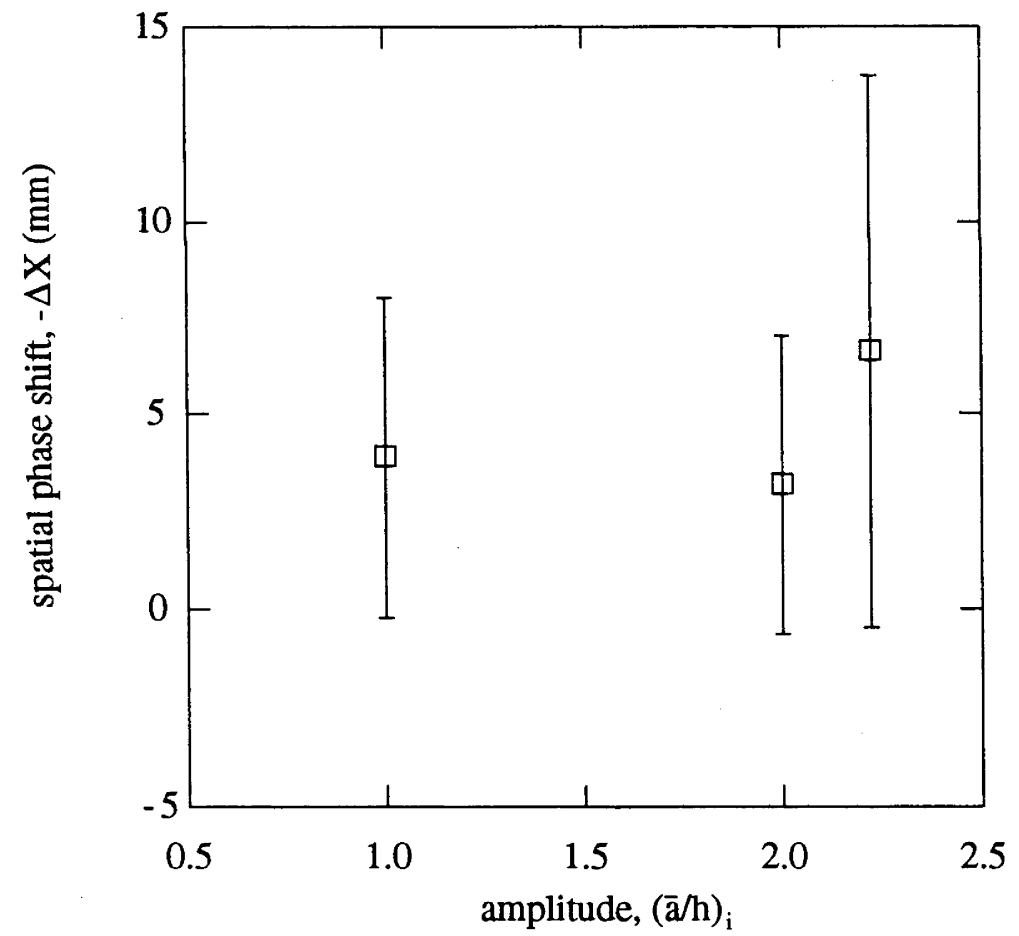

(b)

Figure 2.11: Phase shift results. (a) The trajectory followed by the $X=80 \mathrm{~cm}$ wave during reflection. Extrapolating straight lines fitted to the incident and reflected trajectories indicates that the wave suffered a negative spatial phase shift, that is, appeared to reflect from a virtual endwall behind the real endwall or, alternatively, appeared to hesitate at the endwall. (b) Spatial phase shift vs. incident wave amplitude. The estimation procedure produced large errors, but evidence for phase shifts is convincing with the point estimates indicating that all waves suffered similar delays. 
outgoing waves subsequently collapsed outwards as smaller secondary waves.

As shown in figure 2.13, for waves of different amplitudes the behaviour was modified by the exchange of fluid from the larger incident wave to the smaller incident wave such that the leftward and rightward propagating waves had the same amplitudes before and after the collision. This transfer of fluid was required to satisfy conservation of momentum. Furthermore, although the trapped fluid was principally reflected during head-on collisions, the waves themselves behaved like solitons in preserving their identity while they passed through each other.

\subsection{Summary and conclusions}

An experimental investigation of deep-water internal solitary waves has been presented. At small amplitudes the waves carried energy and momentum, whereas at sufficiently large amplitudes the waves also entrained, mixed and then ejected fluid. The small-amplitude waves were well described by inviscid weakly nonlinear theory (Benjamin; Davis \& Acrivos) but, as expected, the theory failed to predict the behaviour of the large-amplitude waves. However, numerical solutions for inviscid waves (Tung et al.) were consistent with the observed behaviour of large-amplitude waves, and this implies that, despite the presence of closed streamlines, such waves can be accounted for by higher order amplitude terms without regard to viscous effects. In addition, all waves were stable during wave-boundary and wave-wave interactions.

Several avenues for future work were identified. First, here the maximum amplitude of waves was an artefact of the generation technique and interface characteristics, and experiments should be conducted to find out if there is a critical amplitude above which waves are unstable. This question could possibly be answered using a strongly convergent channel. Second, a mode-1 wave closely followed each mode-2 wave, and it remains to be determined whether these waves were radiated from the main wave or were an artefact of the generation technique. A feasible test would be to compare the attenuation of the mode- 2 waves with that expected if downstream wave-radiation were the dominant dissipation mechanism. This approach would also be useful in clarifying whether dissipation occurred through viscous stresses, turbulent mixing or wave-radiation. Finally, the finding that both the wavelength and wavespeed of large-amplitude waves increased linearly with increasing amplitude suggests that a similarity solution exists for these waves. Whilst an appropriate approach to find such a solution is unclear, the fact that numerical solutions for inviscid waves were consistent with the observed behaviour of large-amplitude waves implies that it is valid to neglect viscous effects.

Figure 2.12: Head-on collision between two waves of equal amplitudes. The behaviour was similar to a reflection, with the fluid trapped within each wave reversing direction and being transported away from the collision along the path by which it approached. 

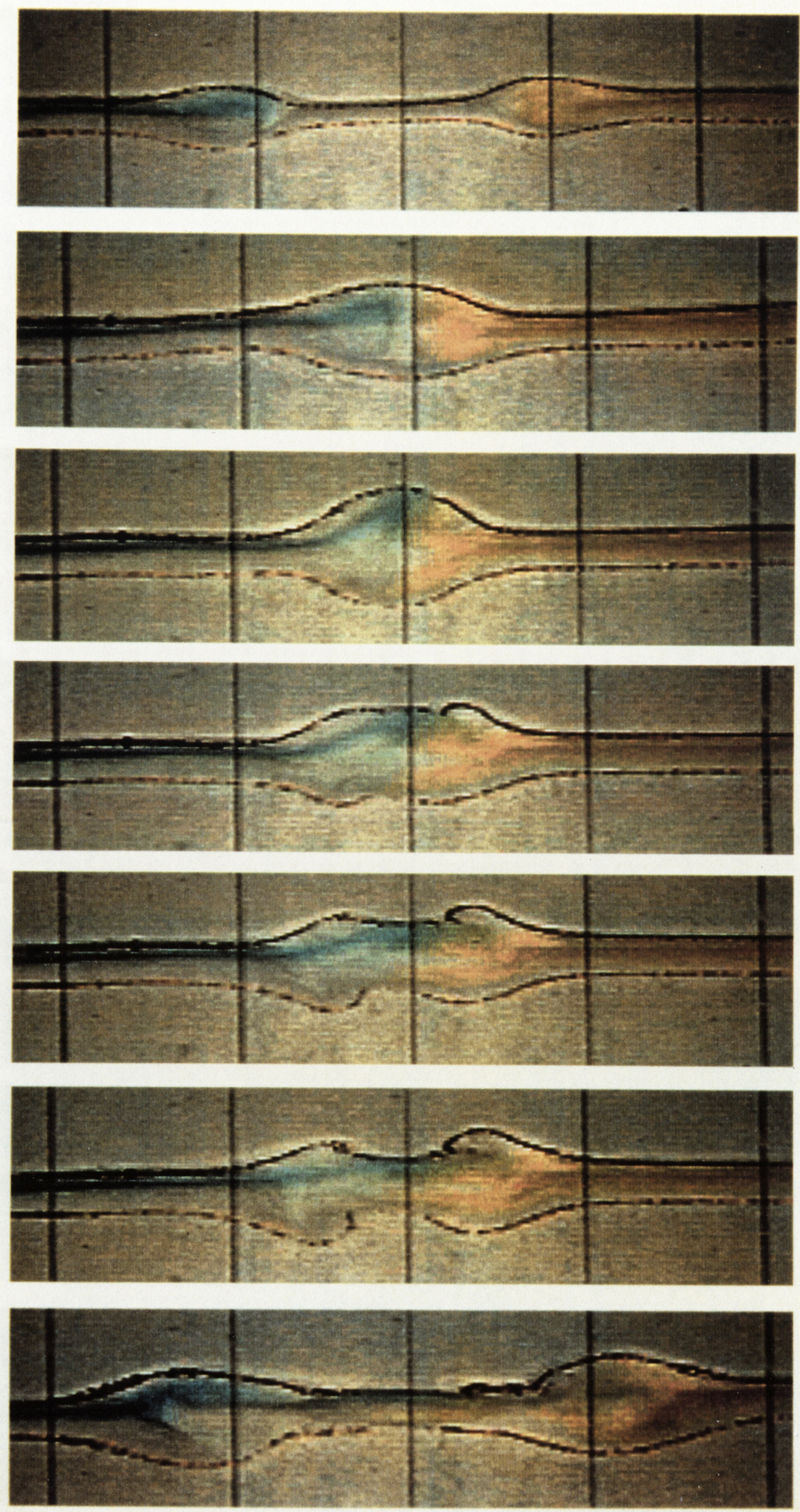

Figure 2.12: Head-on collision between two waves of equal amplitudes. 
Figure 2.13: Head-on collision between two waves of different amplitudes. Whilst most of the trapped fluid was reflected, a small quantity of fluid was exchanged from the larger incident wave to the smaller ingoing wave such that the leftward and rightward propagating waves had the same amplitudes before and after the collision. 

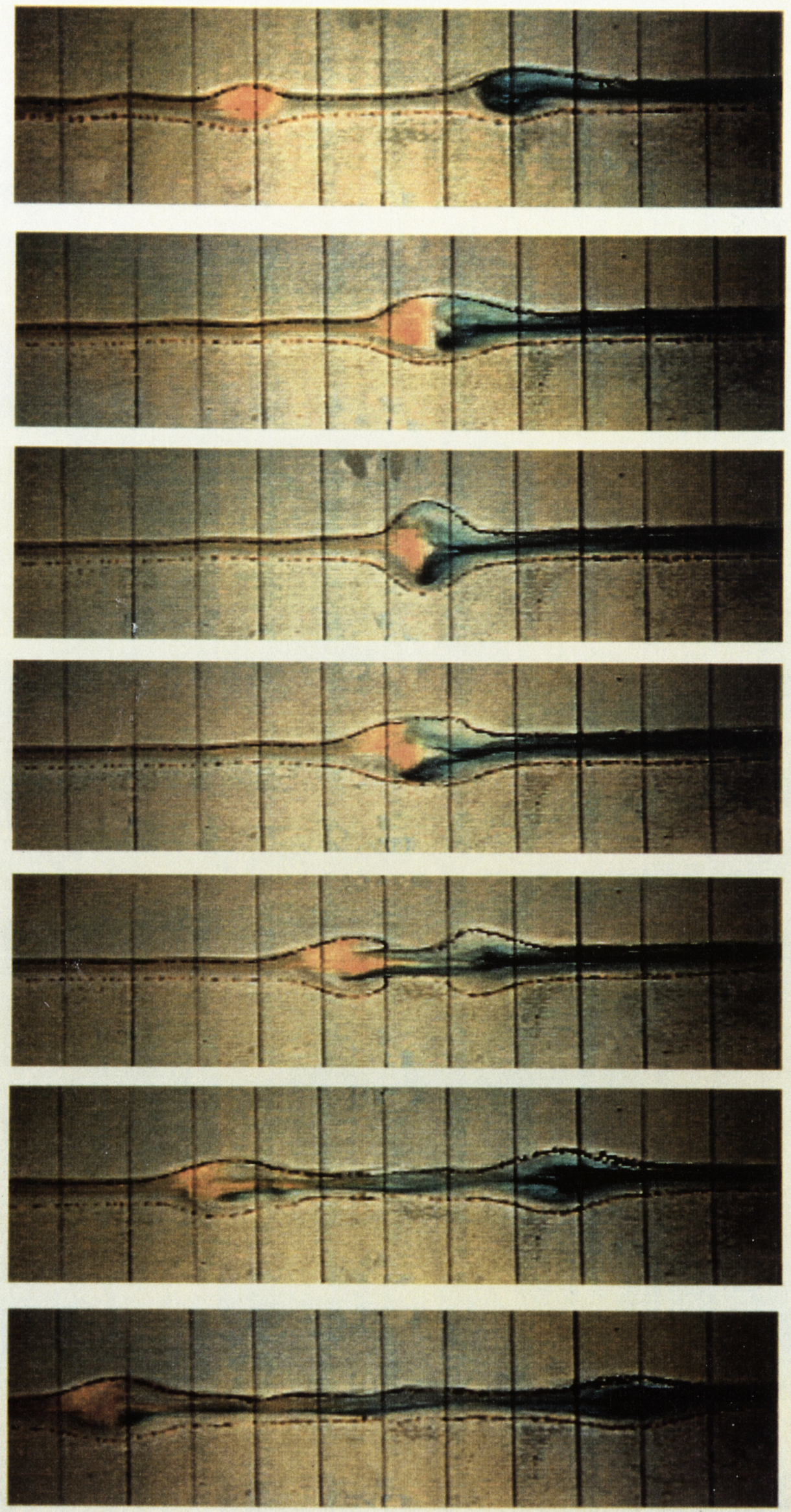

Figure 2.13: Head-on collision between two waves of different amplitudes. 


\title{
Chapter 3
}

\section{The coupling of waves and convection in rectangular channels}

\author{
The laws of gravity are very, very strict \\ And you're just bending them for your own benefit \\ - She's got a new spell, Billy Bragg (1992)
}

\subsection{Introduction}

Double-diffusive convection occurs when a fluid contains two or more components which diffuse at different rates and which make opposing contributions to the vertical density gradient. In such systems instabilities develop even when the initial density gradient is stable because diffusion, a stabilizing mechanism in single component convection, acts to release the potential energy in the unstably stratified component. The exact form of the convection however, depends on whether the faster or slower diffusing component is unstably stratified. When the faster diffusing component is unstably stratified, an interface consisting of a stable 'diffusive' core between unstable boundary layers forms, and drives two convecting layers. Conversely, when the slower diffusing component is unstably stratified, the interface takes the form of an array of thin vertical counterflowing 'fingers'. Both types of convection are ubiquitous. The review articles of Turner $(1974 ; 1985)$ and Huppert \& Turner (1981) report numerous oceanographic observations, as well as examples from such diverse fields as astrophysics, atmospheric physics, geology, liquid natural gas storage, solar pond heat reservoirs and materials science.

Turner (1965) performed the first experiments for the diffusive case, measuring the heat and salt fluxes across an interface formed by heating a stable two-layer salt stratified fluid from below. He used dimensional arguments to show that the fluxes depend on the $4 / 3$ power of the fractional density difference across the interface due to heat $(\alpha \Delta T)$, together with a dependence on the density-anomaly ratio $R_{\rho} \equiv \beta \Delta S / \alpha \Delta T$, where $\beta \Delta S$ is the fractional density difference due to salt. Interpreting measurements within this framework, Turner found that at low $R_{\rho}$ interfacial transport was dominated by turbulent mixing, whereas at high $R_{\rho}$ molecular diffusion through the interfacial core accounts for the whole flux of each component. Following this, Shirtcliffe (1973) demonstrated that a diffusive interface can also form between two concentrated solutions at the same temperature.

This study considers the "systematic interfacial wavemotion coupled to largescale convection in the layers" which occurs when a layer of salt solution lies above a layer of sugar solution (Turner \& Chen, 1974). Preliminary experiments showed 
that in this case the convection spontaneously generated large-amplitude 'waves' on the interface. These waves corresponded to a local thickening of the interface and produced horizontal variations in the interfacial buoyancy flux, which in turn organized otherwise random convective motions by forcing circulations on the scale of the distance between waves. Subsequent interactions between the waves and convection led to a rapid decrease in the number of waves and an increase in the scale of the convective circulations. In turn, the waves themselves persisted for unusually long times owing to energy input from the organized convection. Similar 'wave-convection coupling' was also observed for cold fresh water above hot salt water and glycerol above potassium carbonate water, as well as for different tank and cell geometries.

To simplify the investigation, analysis was restricted to flows carried out in rectangular channels with a single aqueous salt-sugar interface separating two layers of equal depth. A single wave formed once the system evolved to a critical value of the density-anomaly ratio, and propagated back-and-forth along the channel, organizing the convection in each layer into two cells which oscillated in length from zero to the full length of the channel. This cyclic flow continued for about 5 hours, during which time the average thickness of the interface increased, the buoyancy fluxes and convective velocities decreased, and in turn the wavespeed decreased. The dependence of wavespeed on the layer properties and channel dimensions was successfully predicted by assuming that effective coupling requires a matching of the wave and convection speeds, and that the system selects waves of an amplitude for which this resonance can occur.

The structure of this chapter is as follows. Section 3.2 reviews work on the diffusive interface and then $\S 3.3$ describes the experiments. This is followed in $\S 3.4$ by a description of flows carried out in rectangular channels with a single aqueous salt-sugar interface separating two layers of depth $H=11.5 \mathrm{~cm}$, and $\S 3.6$ and 3.7 present measurements of the wave-convection coupling and interfacial fluxes for these flows. Section 3.8 briefly describes flows carried out with different geometries and solutions.

\subsection{Review of the literature}

\subsubsection{Stability analysis}

In a seminal paper, Stern (1960) considered the stability of a horizontal layer of Boussinesq fluid subject to linear opposing temperature and salt gradients. $\mathrm{He}$ focused on the (finger) case of hot salty water above cold fresh water, but mentioned the (diffusive) case of cold fresh water above hot salty water in a footnote. In both cases instabilities develop because the rapid diffusion of heat relative to salt alters the buoyancy of displaced parcels of fluid.

Veronis $(1965 ; 1968)$ was the first to investigate linear and finite amplitude stability when the temperature and salinity both increase with depth. Extending earlier dimensional analysis for Rayleigh-Bénard convection, he showed that the behaviour depends on four parameters: the thermal Rayleigh number

$$
R_{\mathrm{T}}=g \alpha \Delta T H^{3} / \nu \kappa_{\mathrm{T}},
$$

the saline Rayleigh number

$$
R_{\mathrm{S}}=g \beta \Delta S H^{3} / \nu \kappa_{\mathrm{T}}
$$


the Prandtl number

$$
\operatorname{Pr}=\nu / \kappa_{\mathrm{T}}
$$

and the diffusivity ratio

$$
\tau=\kappa_{\mathrm{S}} / \kappa_{\mathrm{T}} .
$$

Here $\Delta T$ and $\Delta S$ are the temperature and concentration differences across the layer (defined $>0), \alpha=\rho^{-1}(\partial \rho / \partial T)$ and $\beta=\rho^{-1}(\partial \rho / \partial S)$ are expansion coefficients which give the fractional density changes produced by unit changes in $T$ and $S, H$ is the layer depth, $\kappa$ is the molecular diffusivity, $\nu$ is the kinematic viscosity, and $g$ is the gravitational acceleration. For any given fluid $\operatorname{Pr}$ and $\tau$ are constant, and convection occurs when the thermal Rayleigh number exceeds a critical value which is dependent on the saline Rayleigh number.

Imposing no-horizontal-flux boundary conditions, Veronis found both standing wave and steady state convection. Standing wave solutions occur near onset and consist of convection cells that reverse direction periodically while remaining fixed in the horizontal. This oscillatory behaviour occurs because a parcel of fluid can only rise a finite height before losing buoyancy via the diffusion of heat. However, as the thermal Rayleigh number is increased the convection destroys the stabilizing salt gradient and eventually the motion consists of steady well-mixed cells driven by thin horizontal boundary layers at the top and bottom of the fluid layer.

Knobloch et al. (1986) generalized Veronis's analysis to account for periodic, rather than no-horizontal-flux, boundary conditions, which introduced the possibility of a horizontally translating pattern of convection cells. Indeed, their numerical solutions show that, for a wide range of fluid properties, the linear standing wave pattern of convection cells is unstable and the cells start to drift to the left or right at a constant speed. Figure 3.1 shows the streamfunction and temperature fields for a leftward travelling-wave solution, and it is the lateral displacement of the vorticity and temperature fields that causes them to travel. As the thermal Rayleigh number is increased the speed at which the cells drift decreases until the cells stop, and the motions resemble those of single component convection.

\subsubsection{Formation of a diffusive interface}

The Rayleigh-Bénard models of double-diffusive convection generally assume that the fluid is confined between horizontal boundaries at which either the values or the fluxes of heat $(T)$ and salt $(S)$ are specified. Such boundary conditions lead to mathematically amenable problems, but cannot be attained in the laboratory because there is no satisfactory method of adding or removing salt at boundaries. The simplest experiment to set up is one where a layer of fluid containing a combination of $T$ and $S$ is poured on top of a denser layer containing another combination of $T$ and $S$. In this case, a finger or diffusive interface separates the convecting layers and the system 'runs down' as the potential energy associated with the unstably stratified component decreases.

Turner (1965) performed the first experiments for the diffusive case, heating a stable two-layer salt stratified fluid from below. He observed that a thin density interface forms and produces unstable fluid that drives convection in the upper and lower layers.

Following this, Elder (1969) used Herring's (1963) mean-field equations to investigate this behaviour. The calculations were started with a stable density stratification of two well-mixed layers separated by a sharp interface (figure 3.2a). Diffusion then acts to smooth the component gradients. However, because $\kappa_{\mathrm{T}}>\kappa_{\mathrm{S}}$, the $T$ 

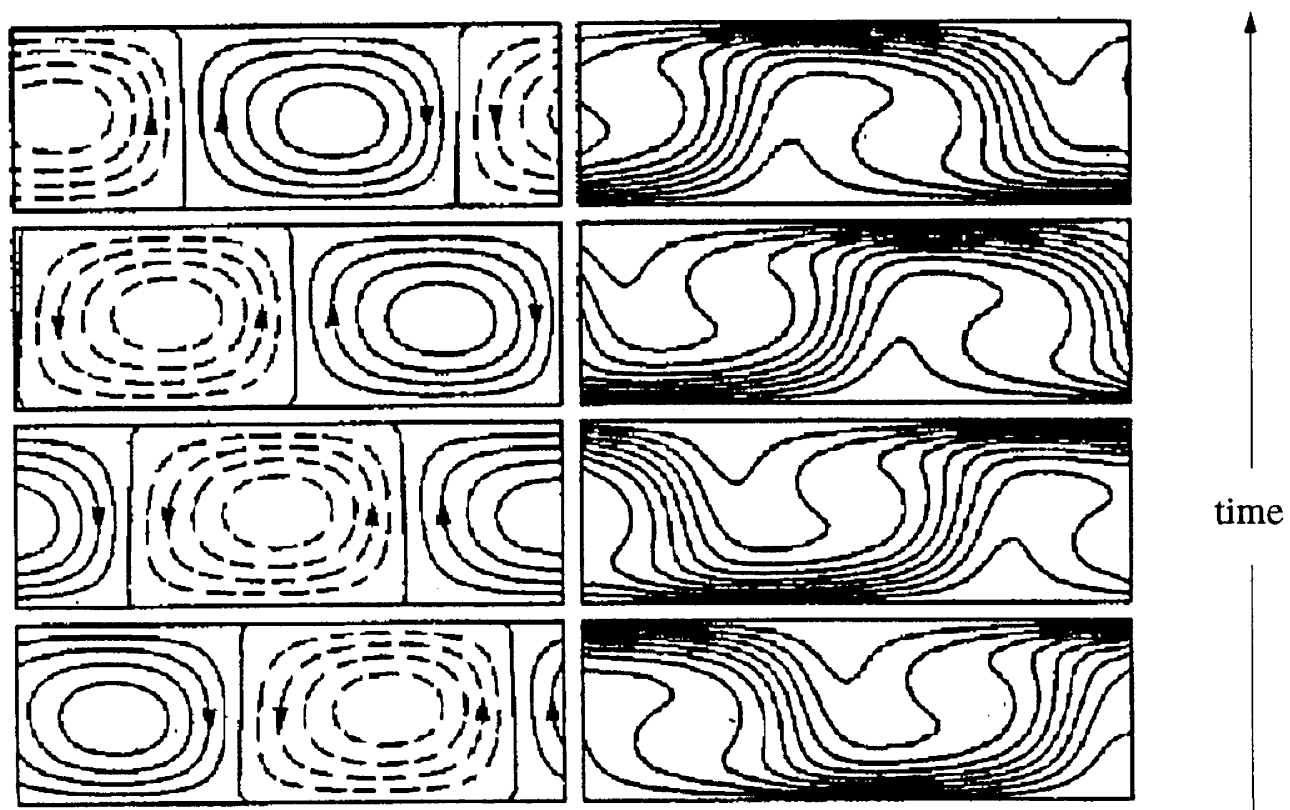

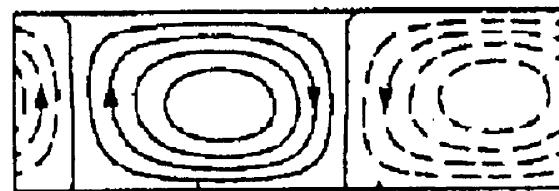

(a)

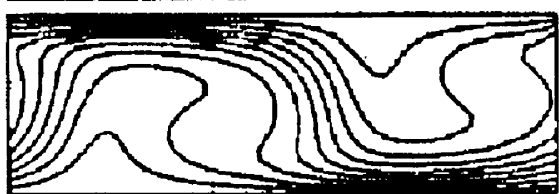

(b)

Figure 3.1: Travelling-wave convection in a double-diffusive system. Theoretical studies of double-diffusive convection have focused on the tractable problem of a horizontal layer of fluid subject to opposing linear gradients of heat and salt. In the diffusive case temperature and salinity both increase with depth, and the first convective motions consist of cells that reverse direction periodically while remaining fixed in the horizontal. However, because salt diffuses more slowly than heat, this flow is unstable and the cells start to drift to the left or right at a constant speed. The $(a)$ streamfunction and $(b)$ temperature fields for a leftward travelling cells are shown, as calculated by Deane, Knobloch \& Toomre (1987). 


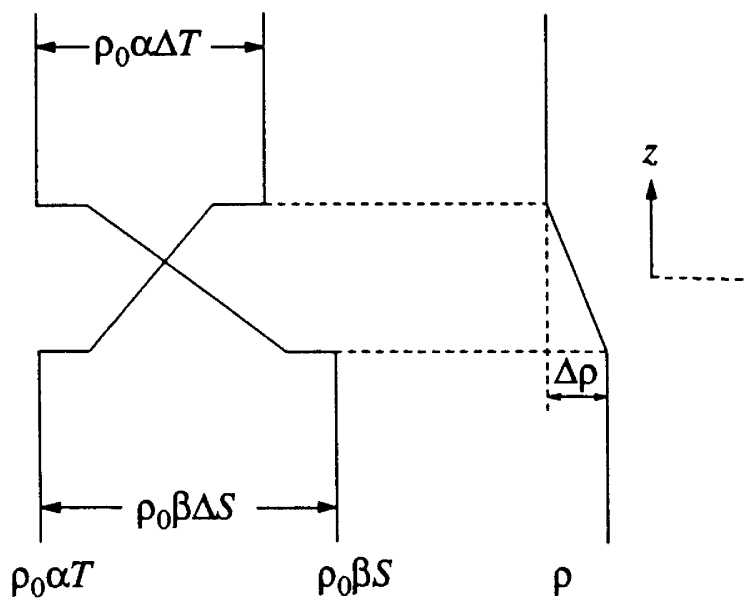

(a)

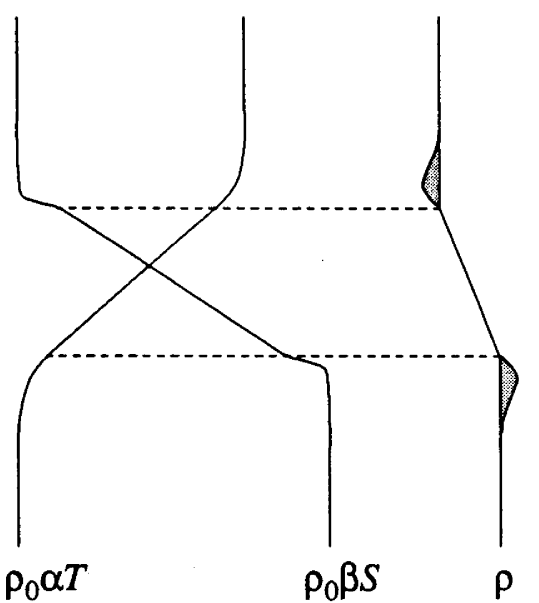

(b)

Figure 3.2: Structure of a diffusive interface. (a) The simplest experiment to set up is one where a layer of fluid containing a combination of $T$ and $S$ is poured on top of a denser layer containing another combination of $T$ and $S$. (b) Diffusion then acts to smooth the component gradients, and because $\kappa_{\mathrm{T}}>\kappa_{\mathrm{S}}$ the $T$ interface thickens more rapidly than the $S$ interface. With the stabilizing effect of the $S$ stratification thus restricted, the net density stratification is no longer stable everywhere. At the upper edge of the interface a positively buoyant boundary layer forms because the density decrease due to the diffusion of $T$ exceeds the density increase due to the diffusion of $S$. Similarly a negatively buoyant boundary layer forms at the lower edge of the interface. The boundary layers eventually break away and drive convection in the layers.

interface thickens more rapidly than the $S$ interface (figure $3.2 b$ ). With the stabilizing effect of the $S$ stratification thus restricted, the net density stratification is no longer stable everywhere. At the upper edge of the interface a positively buoyant boundary layer forms because the density decrease due to the diffusion of $T$ exceeds the density increase due to the diffusion of $S$. Conversely, at the lower edge of the interface a negatively buoyant boundary layer forms because the density increase due to the diffusion of $T$ exceeds the density decrease due to the diffusion of $S$. The boundary layers grow thick enough to become unstable and break away, at which point the interface is thinned by convective mixing.

This type of convection is not restricted to aqueous heat-salt systems; Shirtcliffe (1973) demonstrated that a diffusive interface can also form between two concentrated solutions at the same temperature. In particular, he conducted experiments with salt solution (now the property $T$ that diffuses fastest) above sugar solution $(S)$, for which the ratio of diffusivities is $1: 3$ compared to $1: 100$ for heat and salt.

\subsubsection{Fluxes across a diffusive interface}

Once a diffusive interface forms, it is the coupled $T$ and $S$ fluxes across the interface that are of principal interest. Turner (1965) provided a theoretical framework within which to interpret flux measurements, by first considering the case where the diffusive interface separating a hot salty layer below a cold fresh layer is replaced by a perfectly conducting but impermeable 'solid plane'. Such a change reduces the situation to thermal convection and thus, at high thermal Rayleigh numbers, thin thermal boundary layers on either side of the solid plane drive well-mixed convecting layers. This flow structure suggests that the heat flux depends only on the bound- 
ary layer dynamics and is thus independent of the layer depths. It follows on purely dimensional grounds that the turbulent heat flux across a solid plane is given by

$$
N u \equiv F_{\mathrm{T}}^{\mathrm{SP}} /\left(k_{\mathrm{T}} \Delta T / H\right)^{-1}=C R_{\mathrm{T}}^{\frac{1}{3}}
$$

where $N u$ is the Nusselt number, defined as the actual heat flux divided by the conductive heat flux, and $C$ is a constant.

Generalizing this argument, Turner assumed that the turbulent heat flux across a diffusive interface is independent of the layer depths and therefore given by

$$
N u \equiv F_{\mathrm{T}} /\left(k_{\mathrm{T}} \Delta T / H\right)^{-1}=C R_{\mathrm{T}}^{\frac{1}{3}} F_{\mathrm{T}}^{*}\left(R_{\rho}\right),
$$

if the physical properties $\operatorname{Pr}$ and $\tau$ remain constant. Here the thermal and saline Rayleigh numbers are combined to form the density-anomaly ratio

$$
R_{\rho} \equiv R_{\mathrm{S}} / R_{\mathrm{T}} \equiv \beta \Delta S / \alpha \Delta T
$$

which measures the stability of the interface. The function $F_{\mathrm{T}}^{*}\left(R_{\rho}\right)$ must be determined experimentally and, being equal to $F_{\mathrm{T}} / F_{\mathrm{T}}^{\mathrm{SP}}$, quantifies the effect of the stabilizing salt stratification on the heat flux. Now consider the salt flux. Turner argued that because the convection results from the unstable temperature stratification the salt flux should also depend on the thermal Rayleigh number and the density-anomaly ratio. Applying the above arguments for the salt flux, an expression analogous to (3.6) is derived, which implies that the ratio of fluxes depends only on the density-anomaly ratio:

$$
\beta F_{\mathrm{S}} / \alpha F_{\mathrm{T}} \equiv R_{f}\left(R_{\rho}\right)
$$

Interpreting measurements within this theoretical framework, Turner identified two distinct flux regimes. The first was for $R_{\rho}<2$, where the flux ratio increased as the density-anomaly ratio decreased $\left(R_{f} \rightarrow 1\right.$ as $\left.R_{\rho} \rightarrow 1\right)$ and the heat flux exceeded the solid plane value $\left(F_{\mathrm{T}}>F_{\mathrm{T}}^{\mathrm{SP}}\right)$. The second was for $R_{\rho} \geq 2$, where the flux ratio was constant $\left(R_{f}=0.15\right)$ and the heat flux was inhibited by the stabilizing salt stratification $\left(F_{\mathrm{T}}<F_{\mathrm{T}}^{\mathrm{SP}}\right)$. The existence of these 'variable' and 'constant' $R_{f}$ regimes was confirmed by Crapper (1973), but the exact onset criterion and flux parameterizations remain ill-defined (Crapper, 1973; Marmorino \& Caldwell, 1976; Taylor, 1988). Furthermore, Takao \& Narusawa (1980) observed similar behaviour for other heat-solute systems. However, for the aqueous $\operatorname{salt}(T)$-sugar $(S)$ system, Shirtcliffe (1973) found that the flux ratio was constant $\left(R_{f}=0.60\right)$ down to the smallest values of $R_{\rho}$ that could be achieved experimentally, and showed that the component gradients through the interface are those required if molecular diffusion was to account for the whole flux of each component.

The transition from low to high heat fluxes at $R_{\rho}=2$ was initially associated with the interface dynamics changing from those of a solid plane to those of a free surface. Turner noted that, unlike a solid plane, a free surface supports disturbances that increase its surface area and bring fluid elements of greater temperature contrast together. Furthermore, Huppert (1971) noted that the zero velocity condition for a solid plane is a much stronger constraint on the convective motions than the zero stress condition for a free surface. However, although these effects can explain the excess heat flux, neither can account for the flux ratio variations also characteristic of low $R_{\rho}$. Realizing that the limit $R_{f} \rightarrow 1$ means that all the heat and salt are transported by the same fluid elements, Crapper recognized that both the 
increased heat flux and flux ratio are consistent with turbulent mixing being the dominant transport mechanism. The fact that turbulent mixing is important for variable flux ratio regimes was confirmed by Linden (1974), who successfully modelled Turner's measurements by simply adding empirically determined turbulent mixing and double-diffusive flux contributions.

Linden \& Shirtcliffe (1978) proposed a time-independent mechanistic model of double-diffusive transport across an interface. The interface was modelled as having a stably stratified core, through which transport occurs by diffusion, and two identical marginally unstable boundary layers, one above and one below the core, from which fluid plumes break away driving convection in the layers (figure 3.2). In accordance with Howard's (1966) postulate for high Rayleigh number thermal convection, it was assumed that these boundary layers break down in a periodic fashion; the boundary layers grow by diffusion until they become thick enough to be unstable, the entire boundary layer then breaks away and new boundary layers form. Furthermore, this process was in steady state, that is, over each cycle the diffusive fluxes through the core are equal to the convective fluxes from the boundary layers, and each cycle starts with the same $T$ and $S$ stratifications. To obtain analytical results, it was also necessary to assume that the gradients through the core are linear and remain constant, and that the core is much thicker than the boundary layers.

In this manner, the model predicted how the double-diffusive fluxes and interface thickness depend on the component concentrations and physical properties of the two layers. The flux ratio was found to remain constant

$$
R_{f}=\tau^{\frac{1}{2}}
$$

a result first derived by Veronis (1968) for double-diffusive convection driven by fluxes through a horizontal boundary, and explained by C. Rooth in terms of the instability of a double-diffusive boundary layer. This result is also consistent with constant flux ratio regimes observed in Turner's heat-salt and Shirtcliffe's salt-sugar measurements, but differs from the $R_{f}=0.039 \tau^{-\frac{1}{3}}$ correlation shown in Takao \& Narusawa's measurements of several heat-salt systems. The model also predicted that the functional form of the $T$ flux is

$$
F_{T}^{*}\left(R_{\rho}\right)=\frac{1}{\pi^{\frac{1}{3}}} \frac{\left(1-\tau^{\frac{1}{2}} R_{\rho}\right)^{\frac{4}{3}}}{\left(1-\tau^{\frac{1}{2}}\right)^{\frac{1}{3}}} .
$$

Although this prediction is consistent with the heat-salt and salt-sugar measurements for intermediate $R_{\rho}$, it underestimates the fluxes at other values, even pre-

dicting zero flux for $R_{\rho}=\tau^{-\frac{1}{2}}$. This failure is not unexpected. At low $R_{\rho}$ the assumptions that the core gradients are constant and that the core is much thicker than the boundary layers are inappropriate, whereas at high $R_{\rho}$ the steady state assumption does not hold.

\subsection{Description of the experiments}

In this section the experiments which were carried out in rectangular channels with a single aqueous salt-sugar diffusive interface separating two layers of depth $H=$ $11.5 \mathrm{~cm}$ are described. This configuration was used because it reduced the number of independent non-dimensional parameters to three: the $T$ Rayleigh number, densityanomaly ratio and channel aspect ratio. 


\subsubsection{Experimental setup}

Previous studies have focused on the behaviour of diffusive interfaces formed by heating a stable two-layer salt stratified fluid from below. In this configuration the convective motions of the lower layer are dominated by the buoyancy flux through the bottom boundary rather than that through the diffusive interface. It is therefore not possible for interfacial waves to organize the convection. To overcome this limitation, diffusive interfaces have been formed by simply floating a layer 'heavy in $T$ ' on top of a denser layer 'heavy in $S$ ', so that the convection was entirely driven by the interfacial buoyancy flux. Salt water above sugar water was used. This choice allowed for direct comparison with previous work and for zero boundary flux conditions to be achieved without the need for thermal insulation. Disadvantages were that viscosity was strongly dependent on sugar concentration, and that component concentrations could not be independently determined but had to be inferred from the measurement of two physical properties.

\subsubsection{Independent parameters}

Flow behaviour depended on the component concentrations, physical properties and geometries of the two layers. To facilitate the planning and interpretation of experiments, these variables were combined to form a number of independent nondimensional parameters which govern the system.

As pointed out in $\S 3.2 .1$, the two-dimensional motions of a semi-infinite doublediffusive layer of Boussinesq fluid are governed by the $T$ Rayleigh number (3.1), the $S$ Rayleigh number (3.2), the Prandtl number (3.3) and the diffusivity ratio (3.4). For two layers with similar physical properties the only additional parameter is the depth ratio $H_{\mathrm{u}} / H_{\mathrm{l}}$, where hereinafter the subscripts $\mathrm{u}$ and 1 refer to the upper and lower layers. Finally, in laboratory experiments the effects of the tank geometry must also be taken into account, and tanks of rectangular horizontal cross-section $L \times w$ are characterized by the aspect ratios $L / w$ and $L / H_{\mathbf{u}}$. Hence, provided both layers have similar physical properties, the experimental setup can be defined within a seven dimensional parameter space.

To simplify the investigation several parameters were eliminated. First, further constraints were imposed on the tank and layer geometries. All tanks were long and thin which allowed the dependence on $L / w$ to be ignored; in reality this aspect ratio varied, $3 \leq L / w \leq 12$, but over this range the large-scale motions remained two-dimensional. Also, both layer depths were set to $H=11.5 \mathrm{~cm}$ so that the depth ratio was unity. Second, only the aqueous salt-sugar system was used, and hence variations in physical properties were limited to those resulting from variations in the component concentrations. As a result it was assumed that both layers could be described by $\kappa_{\mathrm{T}}, \kappa_{\mathrm{S}}$ and $\nu$ values for infinitely dilute solutions as well as $\alpha$ and $\beta$ values at the mean concentrations $\bar{T}$ and $\bar{S}$, where hereinafter - denotes a vertically averaged quantity. It follows that the Prandtl number and diffusivity ratio were constant.

In summary, this study examined the dependence of the flow on three nondimensional parameters: the $T$ Rayleigh number, $S$ Rayleigh number and aspect ratio $L / H$. However, in practice the (non-dimensional) fractional density difference $\alpha \Delta T$ was used in place of $R_{\mathrm{T}}$ because under the described conditions all other constituent quantities were constant, and as in other studies the density-anomaly ratio $R_{\rho} \equiv \beta \Delta S / \alpha \Delta T$ was used in place of $R_{\mathrm{S}}$. 


\subsubsection{Apparatus and procedure}

The experiments were carried out in glass channels with horizontal cross-sections ranging from $L \times w=15 \times 5$ to $180 \times 15 \mathrm{~cm}$. Layer depths were chosen to be as large as possible, $H=11.5 \mathrm{~cm}$, in order to produce the slowest rate of change of layer properties. This choice allowed for observations to be made in quasi-steady conditions and for the most accurate determination of component concentrations.

Aqueous salt and sugar solutions were mixed to desired densities and left for a minimum of 12 hours to release any dissolved air and to equilibrate to room temperature $\left(20 \pm 2{ }^{\circ} \mathrm{C}\right)$. In some experiments small quantities of food dye or rheoscopic fluid (fish scales) were added to assist flow visualization. The lower (sugar) layer was then placed in the channel and less dense upper (salt) layer floated on top through one or more diffusers. This procedure took between 5 and 60 minutes depending on the size of the tank. The diffusers were then removed, leaving a free upper surface, and the tank was sealed to prevent evaporation.

Throughout each experiment $2 \mathrm{ml}$ samples were simultaneously withdrawn from the centre of both layers to allow the layer properties to be determined. The sampling interval was chosen to account for the decrease in fluxes with time, and typically ranged from 5 to 30 minutes. At most $2 \%$ of the total fluid was removed.

\subsubsection{Flow visualization}

The behaviour of the diffusive interface and convecting layers was monitored throughout each experiment. Owing to the complex nature of the flow, several flow visualization techniques were required to produce a comprehensive set of observations.

The standard setup used to visualize the flow is shown in figure 3.3. A 50 Watt slide projector illuminated the tank from the opposite side to that from which observations were made, i.e. the 'back' of the tank. Two large pieces of tracing paper were attached to the front of the tank so as to cover the upper and lower layers but not the interface. This setup produced a two-dimensional 'shadowgraph' of the two layers that revealed the convective motions, and emphasized the large-scale convective circulations and the plumes at the wave-nose. In addition, a thin strip of tracing paper was attached to the back of the tank to act as a diffusing screen behind the interface and thereby allow direct observations of the interfacial region to be made. The stable core of the interface was distinguished as a green region between the yellow upper layer and blue lower layer, and it was possible to observe the time-dependent three-dimensional structure of the interface and sheet plumes. Finally, potassium permanganate crystals were occasionally dropped into the tank and distortion of the initially vertical dye lines noted. This technique was an excellent method of visualizing the large-scale convective circulations when there were several interfacial waves.

To supplement these observations, streak (long-exposure) photographs and timelapse $16 \mathrm{~mm}$ movies were taken. These techniques filtered out the short-time scale motions associated with the plumes and thereby emphasized the long time-scale motions associated with the large-scale convective circulations. Streak photographs were set up by adding a small amount of rheoscopic fluid to both layers and arranging projectors at both ends of the tank to illuminate a thin vertical cross-section of fluid. The resulting photographs showed the large-scale flow pattern of the convecting layers, and on occasions individual streaks were sufficiently distinct for accurate velocity estimates to be made. Movies were made using both the standard and streak photograph setups, and could be replayed at a variety of speeds. 

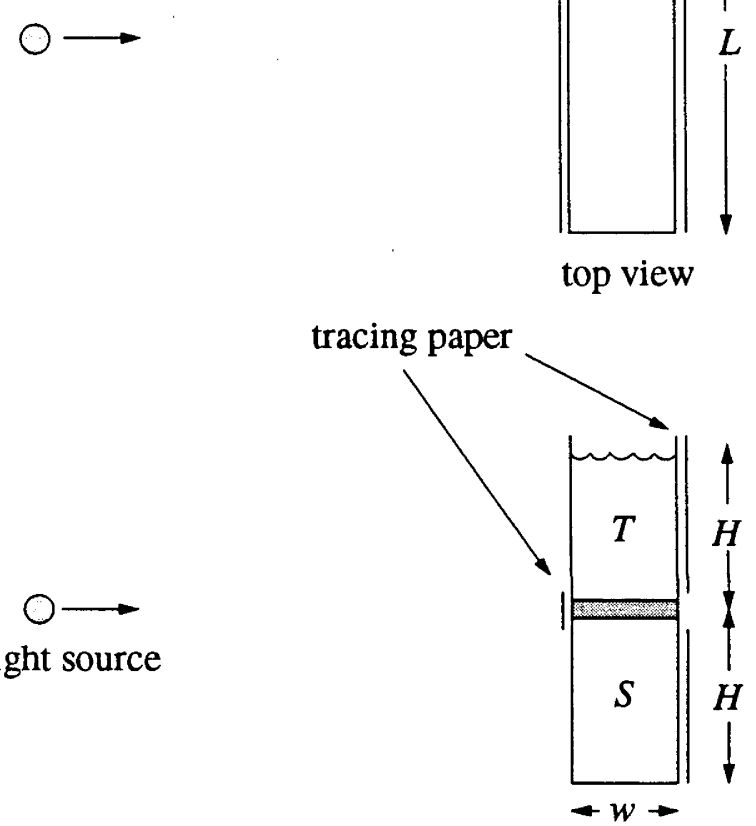

light source
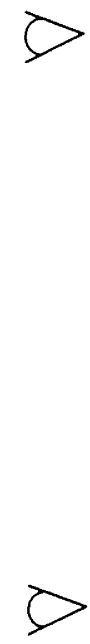

observer

left side view

Figure 3.3: Standard experimental setup. To visualize the flow a 50 Watt slide projector illuminated the tank from the opposite side to that from which observations were made. Two large pieces of tracing paper were attached to the front of the tank so as to cover the upper and lower layers, and a thin strip of tracing paper was attached to the back of the tank to act as a diffusing screen behind the interface. This setup produced a 'shadowgraph' of the convecting layers while allowing for direct observations of the interface. 


\subsubsection{Estimation of salt and sugar concentrations}

The individual component concentrations of aqueous salt-sugar solutions cannot be measured, and must be inferred from measurements of two physical properties that respond differently to the two components. For this purpose, Ruddick \& Shirtcliffe (1979) compiled a list of polynomials relating density, refractive index, conductivity and optical rotation to salt and sugar concentrations (in grams of solute per gram of solution) of aqueous solutions at $20^{\circ} \mathrm{C}$. Here, density and conductivity were used. The density measurements gave an accurate estimate of the interfacial density difference, which was beneficial when comparing the observed waves with gravity current intrusions and internal solitary waves as well as when estimating the interfacial buoyancy flux. Although conductivity tends to be less accurate than optical rotation and is affected by both components, it was more convenient and provided better concentration estimates than those inferred from density and refractive index measurements.

Density was measured at $20.00 \pm 0.01^{\circ} \mathrm{C}$ using a digital densimeter (Anton Parr, K. G. Austria, Model DMA 02C) which gave a precision of $\pm 7 \times 10^{-5} \mathrm{gm} \mathrm{cm}^{-3}$. Conductivity was measured at room temperature using a four wire conductivity probe (Precision Measurement Engineering, USA, Model MSCI-PME 106-300E), while simultaneous temperature measurements were made using a fast response glass thermistor (Fenwal Electronics, USA, Model 0.070 inch diameter). The measured conductivities were normalized to $20^{\circ} \mathrm{C}$ assuming a $2.25 \%$ change per degree Celsius, as is the case for salt solutions subject to small temperature deviations. This system gave a measured precision of $\pm 4 \%$ over the range 5 to $150 \times 10^{-3} \mathrm{mhos} \mathrm{cm}^{-1}$.

In this manner, density and conductivity values at $20^{\circ} \mathrm{C}$ were determined for each sample. Salt and sugar concentrations were then determined by inverting Ruddick \& Shirtcliffe's polynomials using the suggested method. Errors in the polynomials and the inversion process were small compared to the measurement errors. For lower layer solutions (i.e. low $T$, high $S$ ) measurement errors gave an uncertainty of $\pm 0.0004 \mathrm{gm} \mathrm{gm}^{-1}$ for $T$ and $\pm 0.001 \mathrm{gm} \mathrm{gm}^{-1}$ for $S$, whereas for upper layer solutions (i.e. high $T$, low $S$ ) the uncertainties were an order of magnitude larger because of the percentage error in conductivity measurements. Furthermore, with conductivity strongly dependent on the salt concentration and weakly dependent on the sugar concentration, the change in conductivity between successive upper layer samples was less than that for the corresponding lower layer samples. Thus the influence of errors in concealing trends was also greater in the upper layer.

Fortunately, it was found that more accurate estimates of the salt and sugar concentrations in the upper layer could be inferred from the 'measured' lower layer concentrations using the conservation equations. In a two-layer system conservation of mass, salt and sugar requires that

$$
\begin{aligned}
\rho_{\mathbf{u}} H_{\mathbf{u}}+\rho_{\mathbf{l}} H_{\mathbf{l}} & =\text { constant } \\
\rho_{\mathbf{u}} H_{\mathbf{u}} T_{\mathbf{u}}+\rho_{\mathbf{l}} H_{\mathbf{l}} T_{\mathbf{l}} & =\text { constant } \\
\rho_{\mathbf{u}} H_{\mathbf{u}} S_{\mathbf{u}}+\rho_{\mathbf{l}} H_{\mathrm{l}} S_{\mathbf{l}} & =\text { constant },
\end{aligned}
$$

and thus when $\rho, T$ and $S$ values are known for one layer the corresponding values for the other layer can be calculated. Furthermore, if the layer depths, densities and initial concentrations are well constrained, then properties estimated in this manner have similar uncertainties to the properties from which they are calculated. Here measurement errors produced poor estimates of $T_{\mathbf{u}}$ and $S_{\mathbf{u}}$, and therefore $T_{\mathbf{l}}$ and $S_{1}$ were used to calculate more accurate estimates of these properties. The layer 
depths were assumed constant, remaining unchanged to within $\pm 0.5 \mathrm{~mm}$ during experiments with no sampling and decreasing by less than $1.0 \mathrm{~mm}$ during experiments with sampling, and the initial concentrations were well constrained after being inferred from density measurements alone. In all experiments the $T_{\mathbf{u}}$ and $S_{\mathbf{u}}$ estimates calculated in this manner were consistent with those inferred from measurements (figure 3.4).

In summary, accurate estimates of the salt and sugar concentrations in the lower layer were inferred from density and conductivity measurements, but this procedure produced poor estimates of the concentrations in the upper layer because of the percentage error in conductivity measurements. More accurate estimates of the upper layer concentrations were then inferred from the lower layer concentrations using the conservation equations. This technique resulted in similar uncertainties being appropriate for both layers. A formal error analysis suggested uncertainties of $\pm 0.0004 \mathrm{gm} \mathrm{gm}^{-1}$ for $T, \pm 0.001 \mathrm{gm} \mathrm{gm}^{-1}$ for $S, \pm 0.0004$ for $\bar{\alpha} \Delta T$ and \pm 0.01 for $R_{\rho}$, but the experimental data exhibited a precision an order of magnitude smaller.

\subsection{Experimental observations}

A number of preliminary experiments were carried out for familiarization with the experimental procedure and to determine the general nature of the flow. During each experiment the fixed quantities of salt and sugar were redistributed such that $\alpha \Delta T$ and $\beta \Delta S$ decreased, that is, salt moved from the upper to the lower layer and sugar moved from the lower to the upper layer. With $T$ being the more diffusive component $\alpha \Delta T$ decreased faster than $\beta \Delta S$, and therefore $R_{\rho} \equiv \beta \Delta S / \alpha \Delta T$ and $\Delta \rho / \rho_{0} \equiv \beta \Delta S-\alpha \Delta T$ increased (figure 3.15). In response to this homogenization, the fluxes decreased, convection weakened, interface thickened and the flow evolved in a characteristic manner. Over the first hour the convection became progressively more two-dimensional and organized the interface into thick and thin regions. Following this, the thick regions combined to form a single thick region of interface which propagated back-and-forth along the channel, modulating the fluxes and organizing the convection into large-scale circulations. Here observations of the experiments are presented, with measurements given for those started at low $R_{\rho}$ in the $15 \times 5 \mathrm{~cm}$ channel.

\subsubsection{Filling disturbances}

Despite the care taken when floating the upper layer on top of the lower layer, this procedure always generated disturbances. Three types of filling disturbances occurred. First, mixing at the interface produced intermediate density fluid, which was then entrained into the layers at a rate dependent on the strength of the convection. Second, when multiple diffusers were used, it was difficult to maintain equal inflow rates, and horizontal differences in density and composition were set up in both layers. These gradients led to large-scale convective circulations but were soon destroyed by the convection. Finally, while removing diffusers, the interface was occasionally displaced and thereby set into a slow seiching action. Such oscillations were rapidly damped by friction.

Mixing occurred in all experiments; when using diffusers with low inflow rates the upper layer density decreased below that of the inflow causing overturning, whereas with high inflow rates mixing occurred because of the downward momentum of the inflow. The amount of mixing and the subsequent behaviour of the flow depended on 


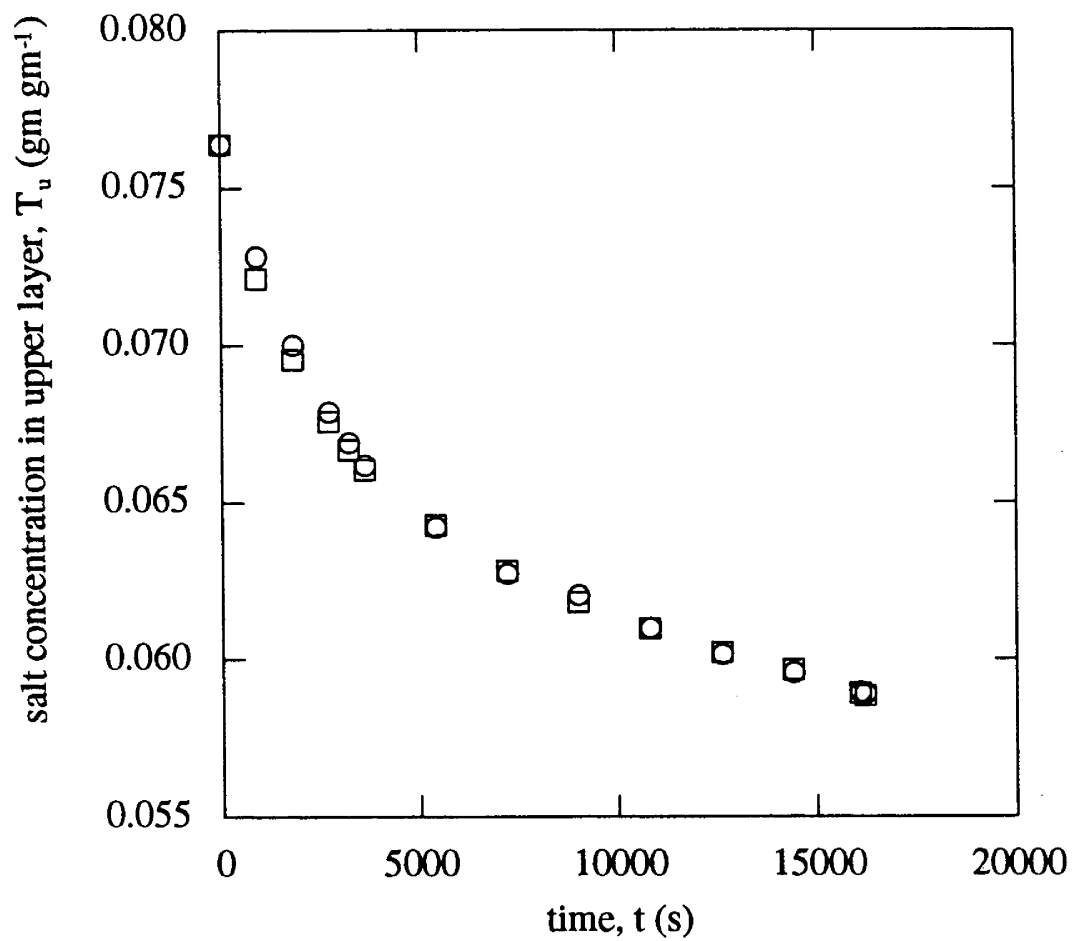

(a)

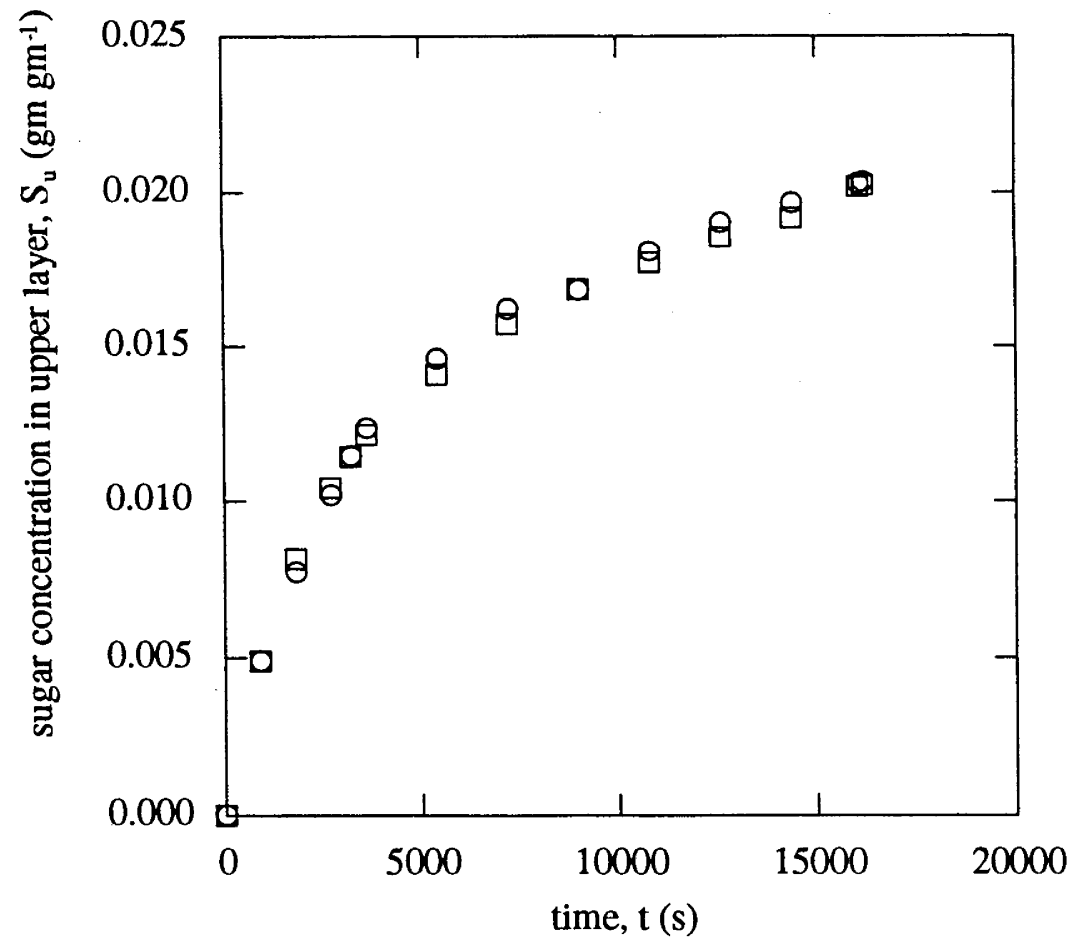

(b)

Figure 3.4: Concentration estimates. (a) Salt and (b) sugar concentrations in the upper layer were determined from inverting density-conductivity measurements $(O)$ and the measured lower layer concentrations using conservation equations ( $\square$ ). Although the two techniques produced consistent estimates, the uncertainties for the estimates derived from measurements were an order of magnitude larger than those for the estimates derived from the lower layer concentrations. This difference occurred because the percentage error in conductivity measurements produced large absolute errors in the upper (salt) layer and small absolute errors in lower (sugar) layer. 


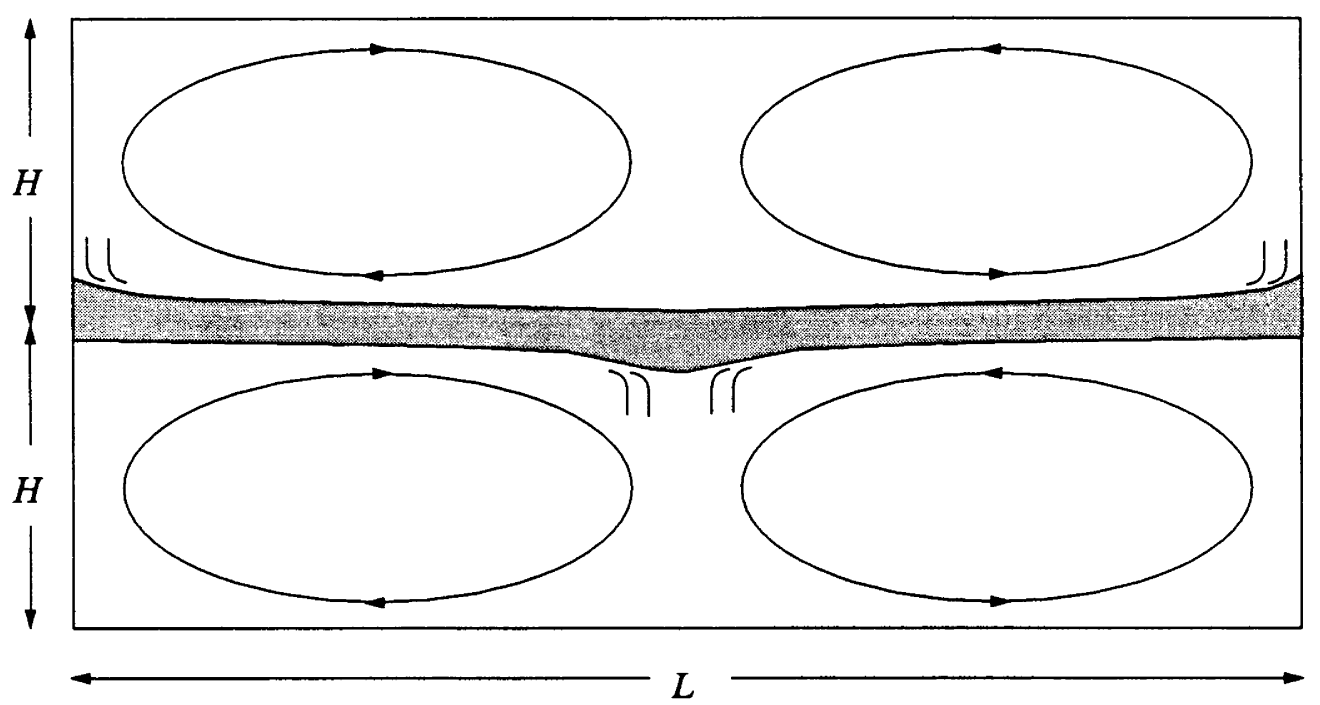

Figure 3.5: Illustration of flow after filling at high $R_{\rho}$. In all experiments mixing occurred while filling, and produced intermediate density fluid which was then entrained into the layers. At high $R_{\rho}$ the convection was too weak to immediately entrain all the mixed fluid, and a uniformly thick stably stratified interface formed and stopped the convection. After about an hour convection restarted and eroded the interface until the flow was indistinguishable from that of experiments started with little mixing. This weak flow consisted of two large-scale counter-rotating cells in each layer; in the upper layer cells swept interfacial fluid to the endwalls from where plumes were released upwards, and in the lower layer the cells swept interfacial fluid to the centre of the channel from where plumes were released downwards. This flow was unique in that the convection was too weak and interface too thick for stress coupling between the layers to be important.

the conditions. At low $R_{\rho}$, the convection was sufficiently vigorous to entrain all the mixed fluid, and thus the interface remained thin $(<1 \mathrm{~mm})$ and there was no start up period in the fluxes. In contrast, at high $R_{\rho}$ the convection could only entrain some of the mixed fluid and a uniformly thick $(10 \mathrm{~mm})$ stably stratified interface formed. A period of inactivity followed, during which diffusion re-established the double boundary layer structure of the interface. Convection then slowly restarted. In general, two large-scale counter-rotating cells formed in each layer, with the upper layer cells sweeping interfacial fluid to the endwalls and the lower layer cells sweeping interfacial fluid to the centre of the channel (figure 3.5). Thus plumes were released from the endwalls into the upper layer and from the centre of the channel into the lower layer, thereby reinforcing the flow. The convection eroded the interface and the fluxes increased, so that within an hour the flow was indistinguishable from that of experiments started with little mixing.

In conclusion, the fact that the flow behaved in the same manner for experiments carried out with different filling techniques and different tank geometries, suggests that the long-term behaviour did not depend on the conditions imposed during filling. For example, experiments $1.6 a, b$ exhibited identical behaviour after both were started with the same solutions using different filling techniques.

\subsubsection{Transient behaviour}

After the mixed fluid was entrained, the fluxes started to decrease, and in turn the convection became weaker allowing the interface to thicken by diffusion. For experiments started at low $R_{\rho}$ this evolution began with a period of transient behaviour 
characterized by three distinct flow regimes.

The first transient flow regime is shown in figure 3.6. This regime occurred when $R_{\rho}<1.10$, and was governed by a balance between the production of stable interfacial core fluid and its entrainment into the layers by the convection. The flow was characterized by a uniformly thin interface $(<1 \mathrm{~mm})$, small three-dimensional convection cells and vigorous three-dimensional convective motions. Sheet plumes emanating from the interface defined the cell boundaries, and ascending and descending plumes were arranged symmetrically. Thus the flow in one layer formed a mirror image of that in the other layer so as to minimize the interfacial shear. Individual plumes were stable for extended intervals because the convection swept the marginally unstable boundary layer fluid produced within cells to the base of existing plumes. However, over time, the cells moved randomly about the channel, expanding, contracting and merging. In general, large cells expanded at the expense of small cells, but once a cell exceeded a critical size the boundary layer fluid broke away before reaching the cell boundary, and several smaller cells formed.

After about 30 minutes the convection became too weak to entrain all the interfacial fluid produced, and within 5 minutes the interface thickened and the flow changed to that shown in figure 3.7. The interface remained thin everywhere except at the base of the plumes, where it was about $2 \mathrm{~mm}$ thick, and the plumes became aligned across the channel forming small two-dimensional cells but continuing to drive three-dimensional motions. This second transient flow regime occurred when $1.10 \leq R_{\rho}<1.13$ and, as such, lasted for about 15 minutes. During this time a large amount of interfacial fluid was produced and all this fluid was swept to the base of the plumes, and the convection thereby organized the interface into thick and thin regions.

The third transient flow regime is shown in figure 3.8 and occurred when $1.13 \leq$ $R_{\rho}<1.15$. The flow was characterized by large thick regions of interface at the base of the plumes, large two-dimensional cells and two-dimensional motions. In general, these interfacial 'lumps' extended across the channel and were about $4 \mathrm{~mm}$ thick and $2-5 \mathrm{~cm}$ long, while elsewhere the interface remained $<1 \mathrm{~mm}$ thick. The variations in the thickness of the interface produced variations in the interfacial gradients of $T$ and $S$, and hence variations in the interfacial fluxes. Thus the thick (small gradient) regions produced less marginally unstable boundary layer fluid and stable interfacial core fluid than the thin (large gradient) regions. Nevertheless, the flow was stable because the stress exerted by the convection stopped the lumps spreading out under the restoring force of gravity. Indeed, over time the lumps were swept back-and-forth along the channel and torn apart at the centre of the channel where the convection was strongest. However, when a lump was swept against an endwall it increased in thickness (and decreased in length) until the convective stresses could no longer balance the restoring force of gravity, at which point the lump collapsed back towards the centre of the channel leaving a thin high flux region behind that reversed the local convective circulation.

Initially the number of interfacial lumps depended on the channel length; increasing from 2 to 10 as the length increased from 15 to $180 \mathrm{~cm}$. As the lumps moved back-and-forth, both head-on and overtaking collisions occurred. The behaviour during a head-on collision is illustrated in figure 3.9. Small changes in either the size of the lumps or strength of the convection destabilized the initial flow so that the lumps converged. As this motion occurred, the size and strength of cells 1 and 4 , which assisted the progress of the lumps, increased at the expense of cells 2 and 3. When the lumps collided they formed a single large thick region. The remaining 
plumes were then swept into the centre of the lump following which cells 2 and 3 disappeared. The lump then increased in thickness until the convective stresses could no longer balance the restoring force of gravity, at which point the lump collapsed outwards forming leftward and rightward propagating lumps. This collapse left a thin high flux region in its wake, allowing cells $\mathrm{B}$ and $\mathrm{C}$ to form.

As a rule, leftward and rightward propagating lumps had the same amplitudes before and after a head-on collision. This behaviour is similar to that for both gravity current intrusion and internal solitary wave collisions in the absence of ambient motions. Furthermore, as there was no discernible change in the convection, the entire leftward and rightward travelling flow patterns may be thought of as passing through each other like solitons, rather than reflecting about an imaginary boundary. In this interpretation, the cells, as well as the lumps, passed through each other according to $[1,2] \Leftrightarrow[C, D]$ and $[3,4] \Leftrightarrow[A, B]$. However, when the cells were of different strengths, the lump moving toward the weaker cell emerged from the collision larger than either of the incident lumps. This process saw large lumps engulf small lumps.

The second type of interaction was an overtaking collision and is illustrated in figure 3.10. In this case, the initial flow was destabilized in such a way that both lumps moved in the same direction (to the left). When the leading (left) lump was slower than the following (right) lump, the size and strength of cells 2 and 3 decreased. This change in the convection hindered the progress of the leading lump and assisted the progress of the following lump, thereby causing the lumps to converge while moving in the same direction. When the lumps collided they formed a single large thick region. The remaining plumes were then swept into the centre of the lump following which cells 2 and 3 disappeared. Unlike head-on collisions, the two incident lumps did not emerge from this collision. Instead the single large lump remained intact and moved to the left or right depending on the strength of the adjacent convection cells.

With large lumps engulfing small lumps during head-on collisions and lumps merging during overtaking collisions, the number of lumps decreased with time. When the final two lumps collided near an endwall, the high fluxes along the thin interface extending to the opposite endwall set up horizontal density gradients within the layers that led to tank-scale convective circulations. The two lumps then merged and propagated back along the channel. This merging occurred about 15 minutes after the start of the third transient flow regime, and signified the end of the transient behaviour.

Figure 3.6: First transient flow regime; ( $a$ ) illustration, (b) direct photograph and (c) streak photograph. This regime occurred when $R_{\rho}<1.10$, and was governed by a balance between the production of stable interfacial core fluid and its entrainment into the layers by the convection. The flow was characterized by a uniformly thin interface $(<1 \mathrm{~mm})$, small three-dimensional convection cells and vigorous three-dimensional convective motions. 
front view

top view
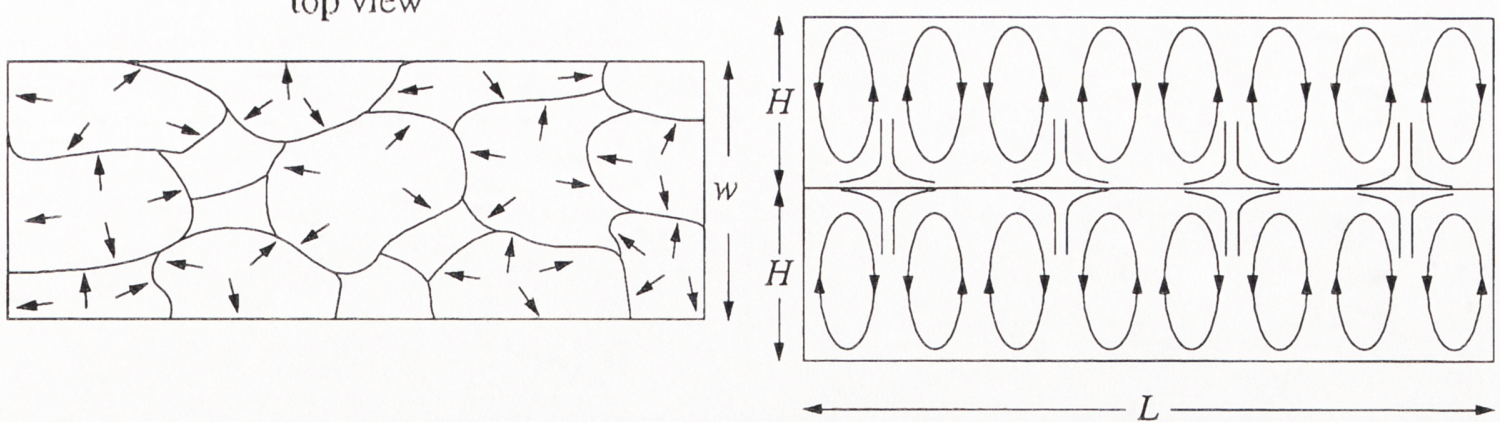

(a)

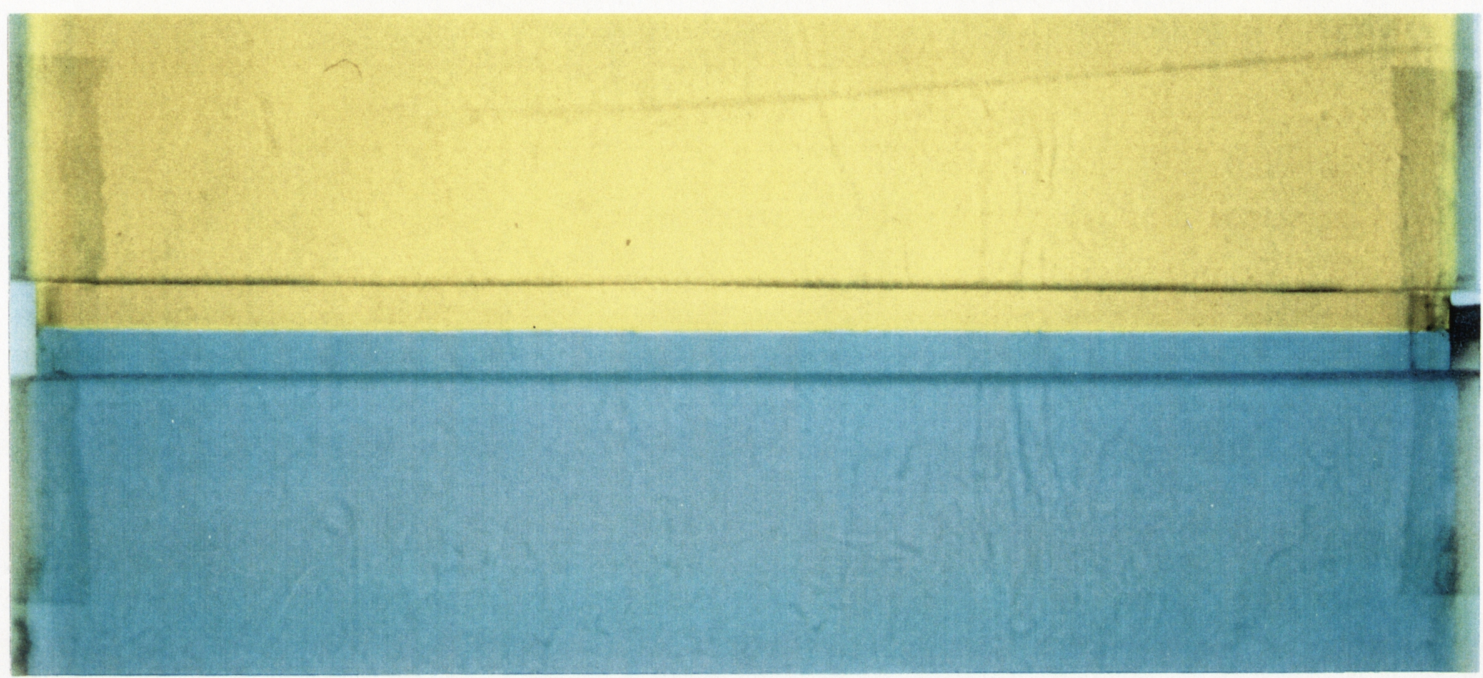

(b)

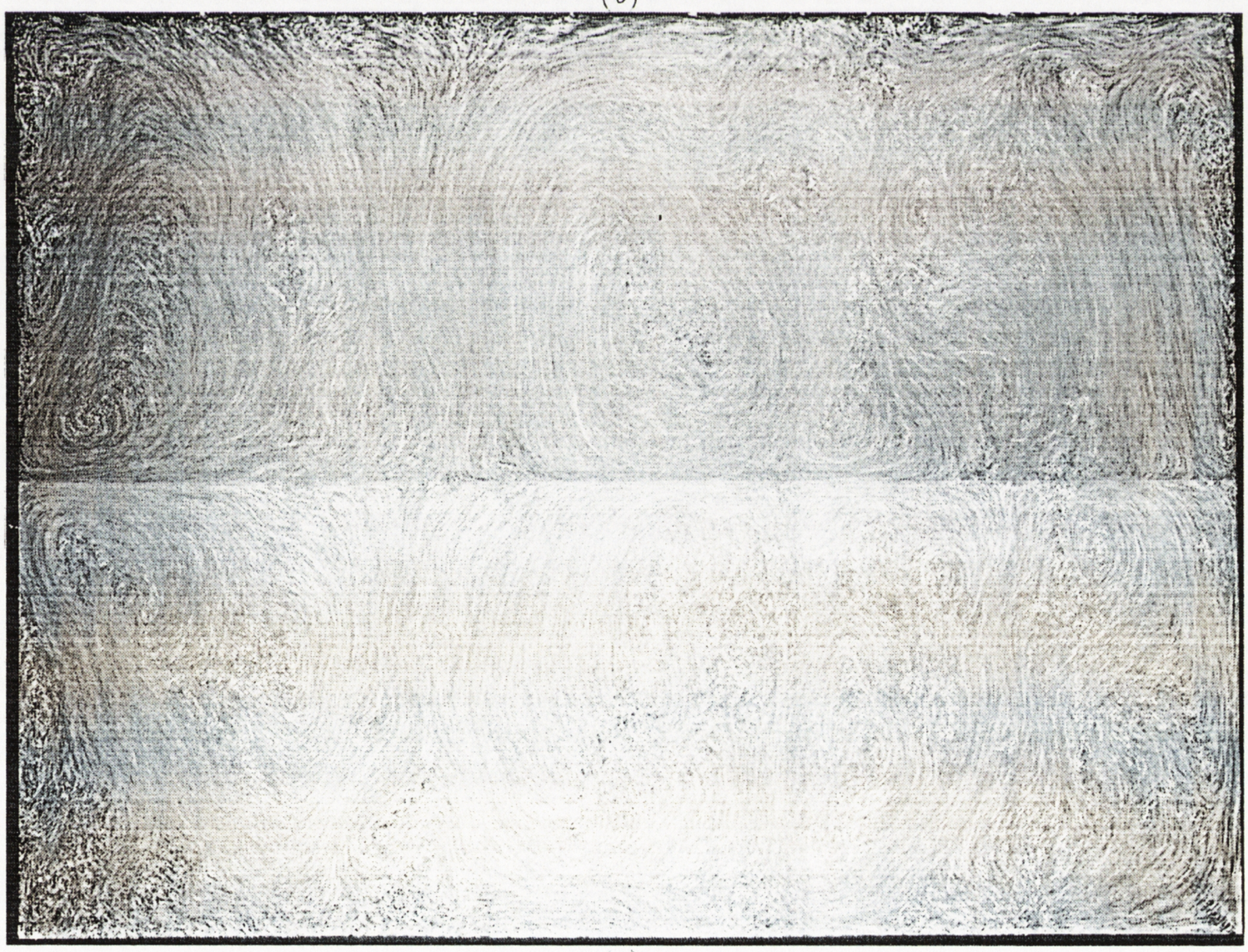

(c)

Figure 3.6: First transient flow regime. 
Figure 3.7: Second transient flow regime; (a) illustration. (b) direct photograph and (c) streak photograph. This regime occurred when $1.10 \leq R_{f}<1.13$ and, as such, started once the convection became too weak to entrain all the interfacial fluid produced. The flow was characterized by an interface which was thin everywhere except at the base of the plumes, small two-dimensional cells aligned across the channel and three-dimensional convective motions. 
front view

top view
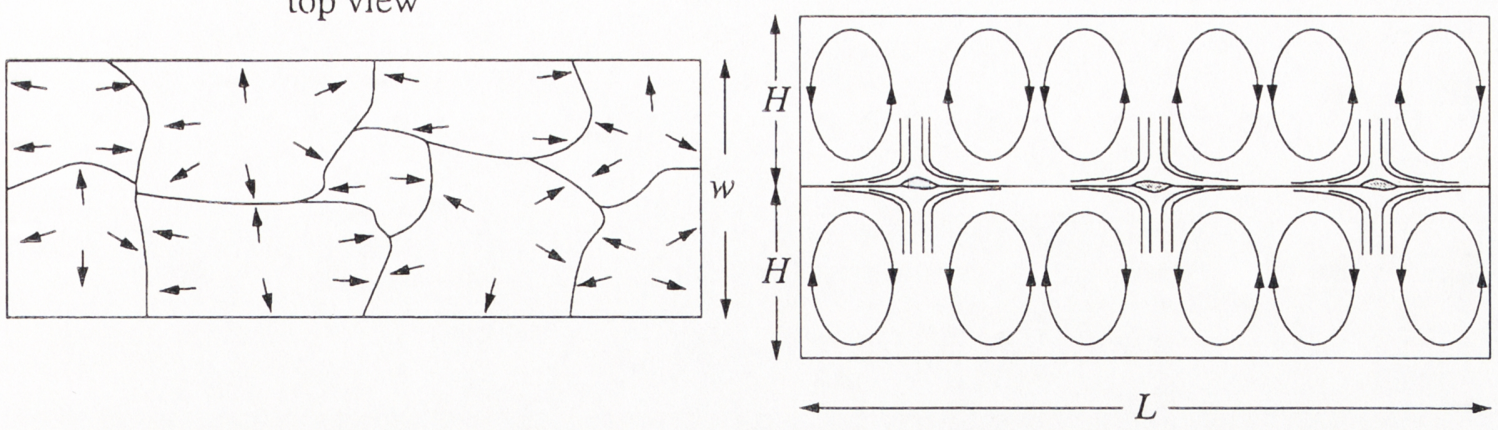

(a)

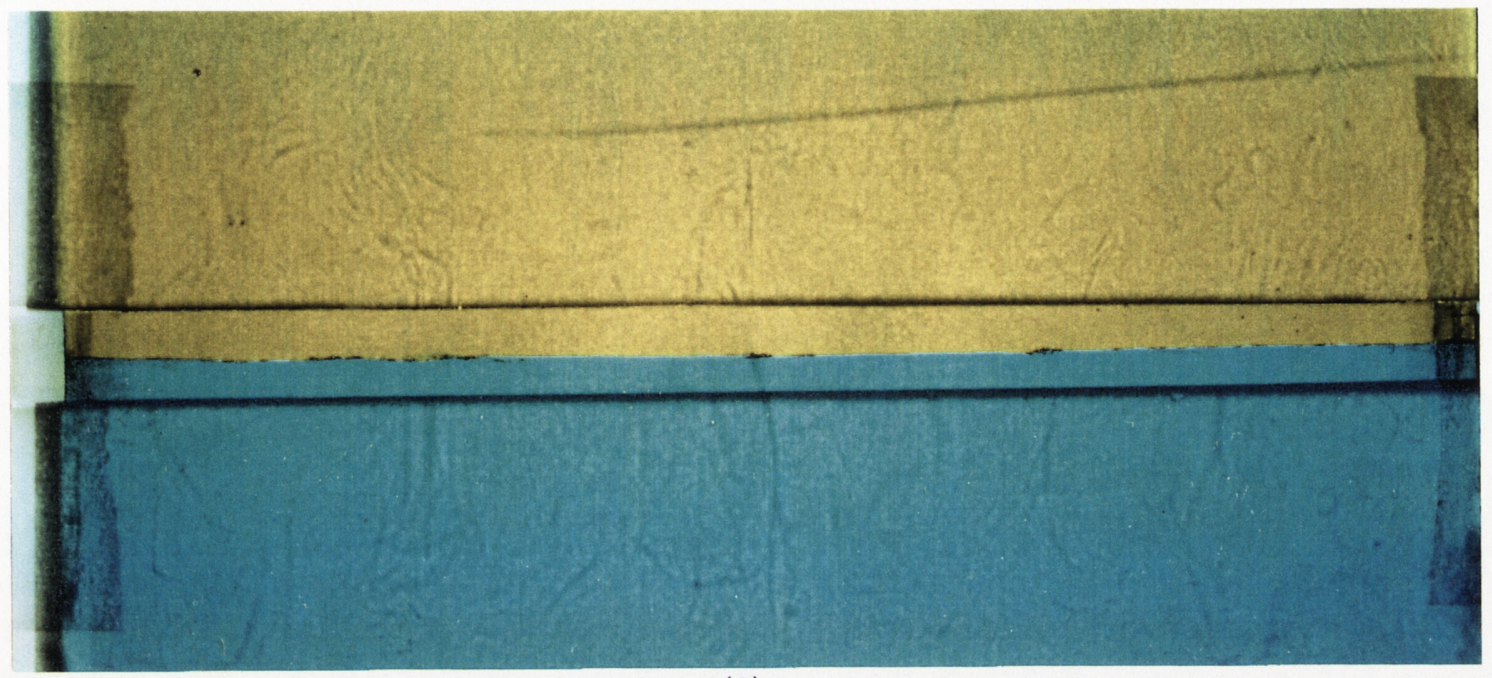

(b)

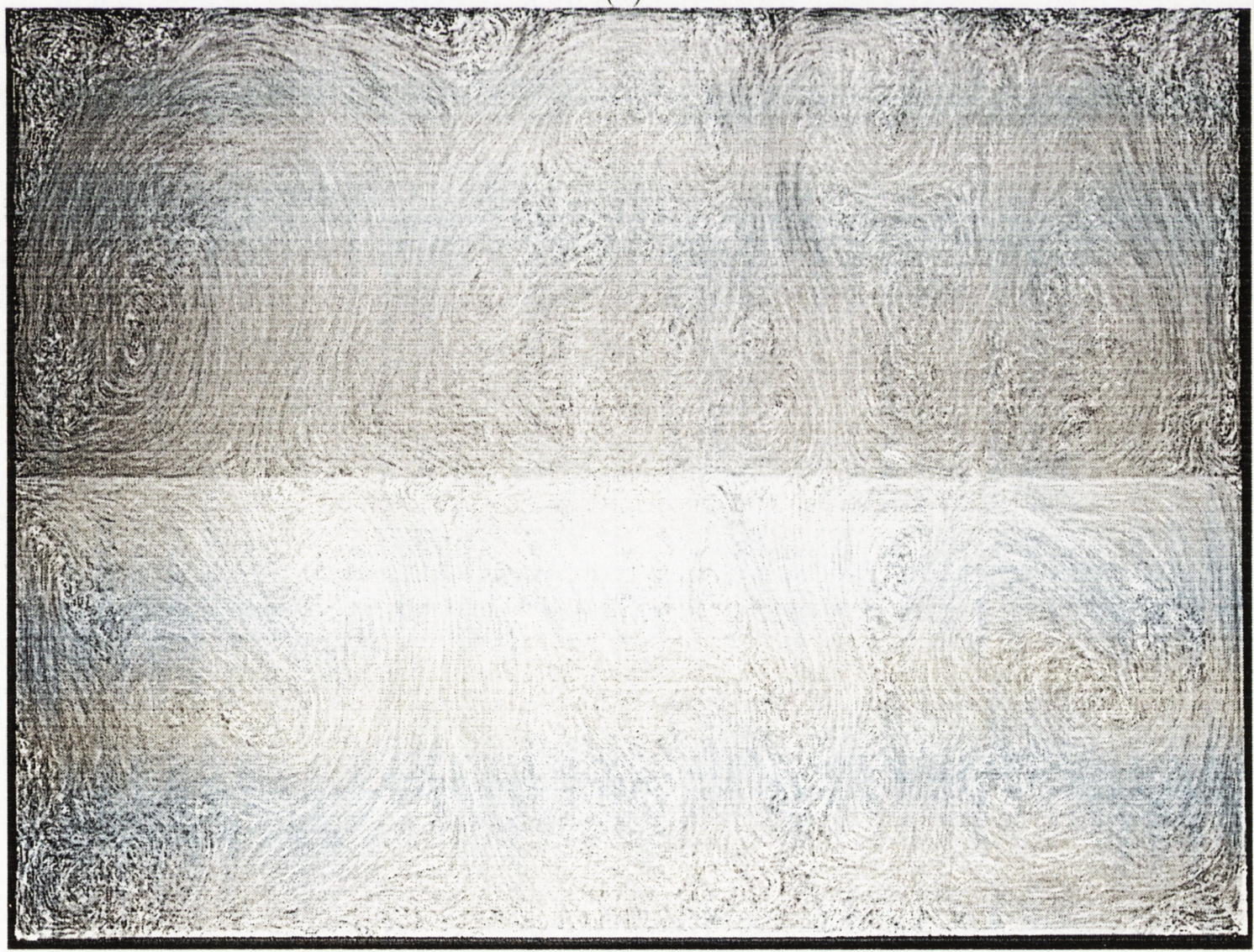

(c)

Figure 3.7: Second transient flow regime. 
Figure 3.8: Third transient flow regime; (a) illustration, (b) direct photograph and (c) streak photograph. This regime occurred when $1.13 \leq R_{p}<1.15$. The flow was characterized by large thick regions of interface at the base of the plumes, large two-dimensional cells and two-dimensional motions. These interfacial lumps were maintained by the stress exerted by the convection and the pressure gradients associated with plume production, and over time were swept back-and-forth along the channel 
front view

top view
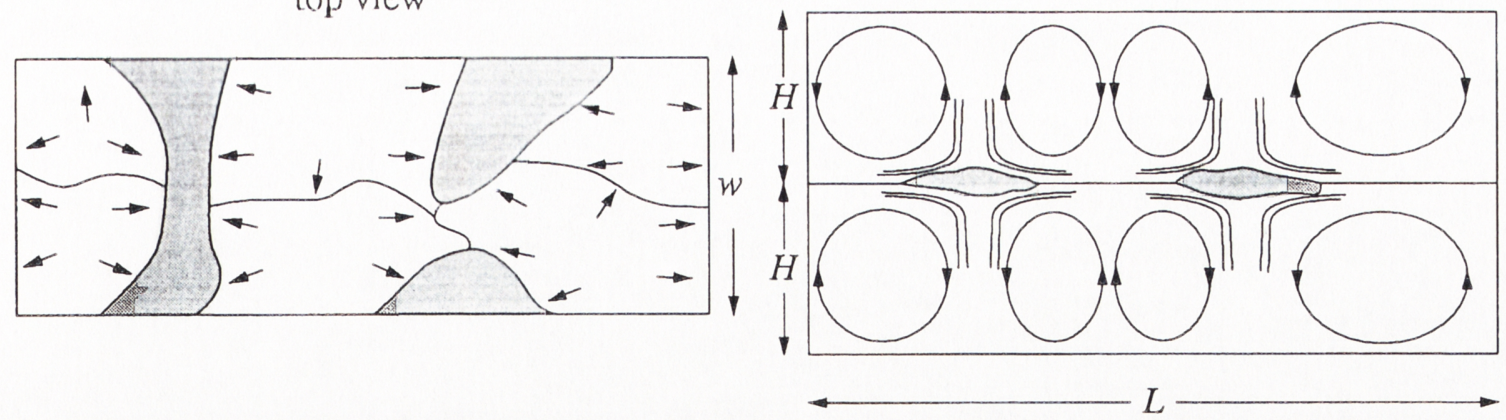

(a)

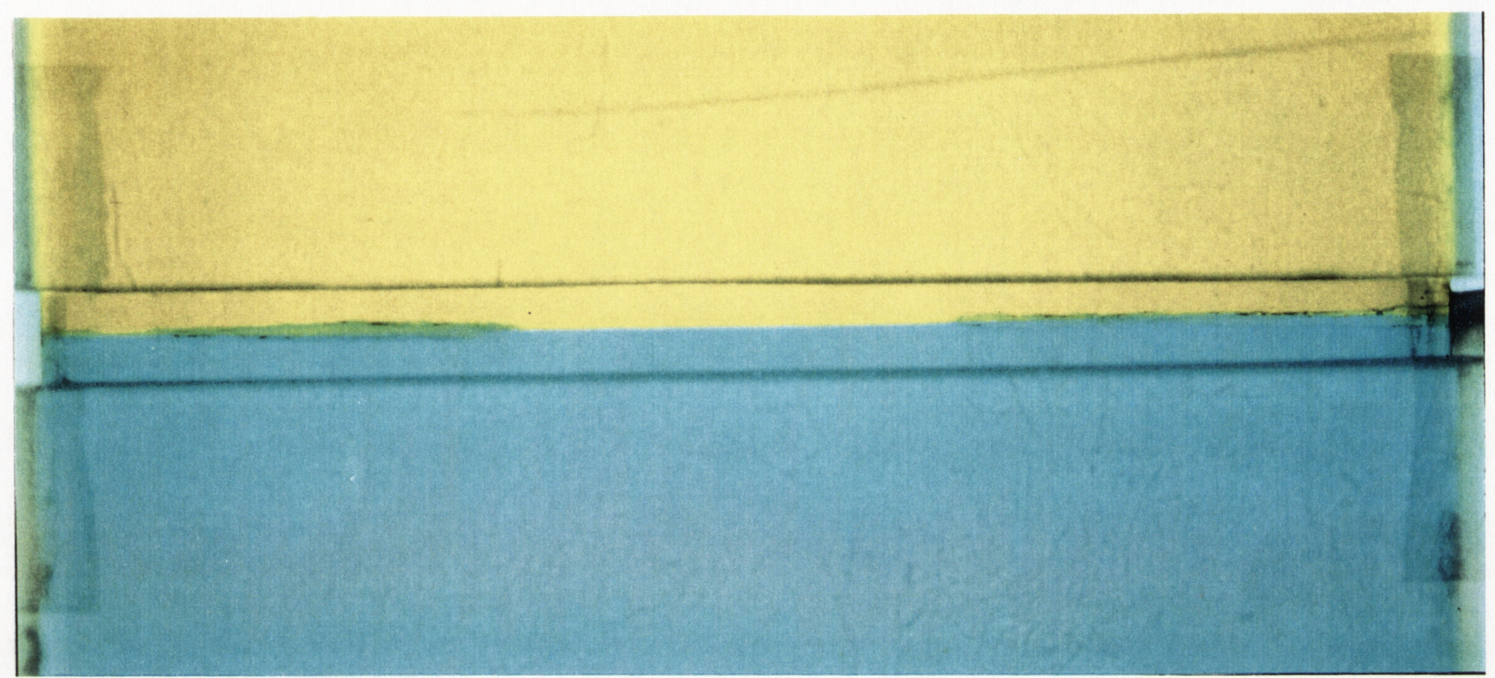

(b)

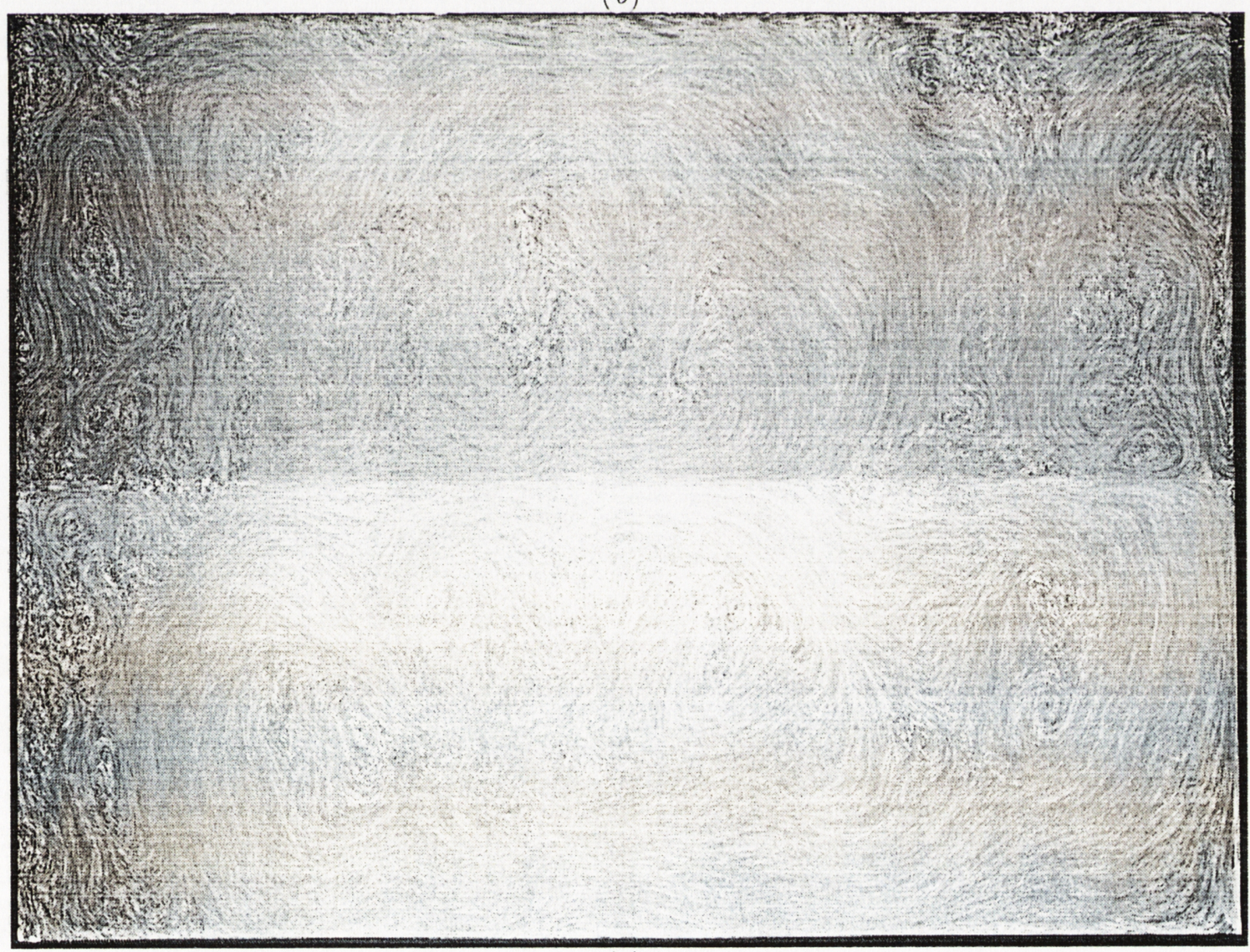

Figure 3.8: Third transient flow regime. 


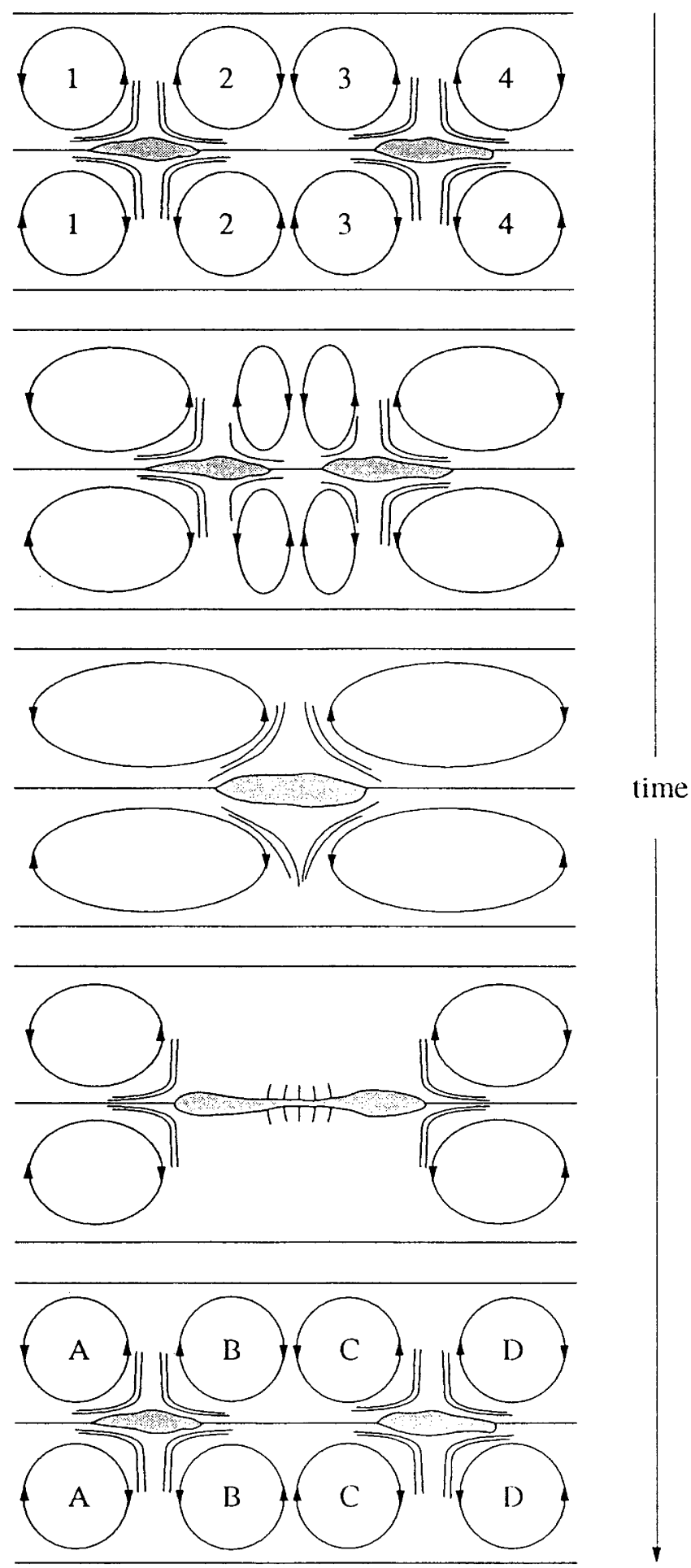

Figure 3.9: Illustration of a head-on collision. As the two lumps converged, the size and strength of cells 1 and 4, which assisted the progress of the lumps, increased at the expense of cells 2 and 3 . When the lumps collided, they formed a single large thick region. The remaining plumes were then swept into the centre of the lump following which cells 2 and 3 disappeared. This lump increased in thickness until the convective stresses could no longer balance the restoring force of gravity, at which point the lump collapsed outwards forming leftward and rightward propagating lumps. Cells B and $\mathrm{C}$ then formed. 

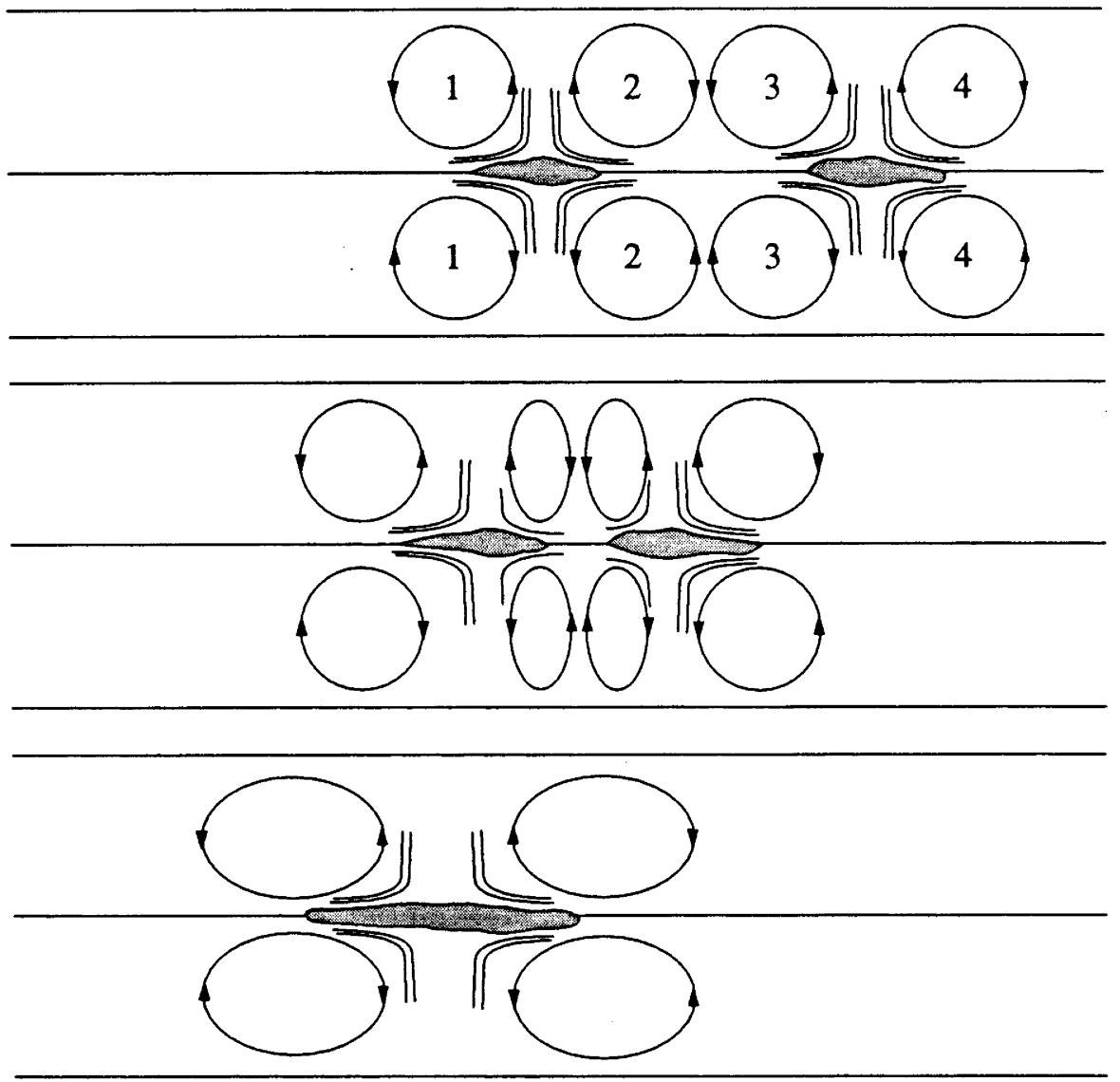

time
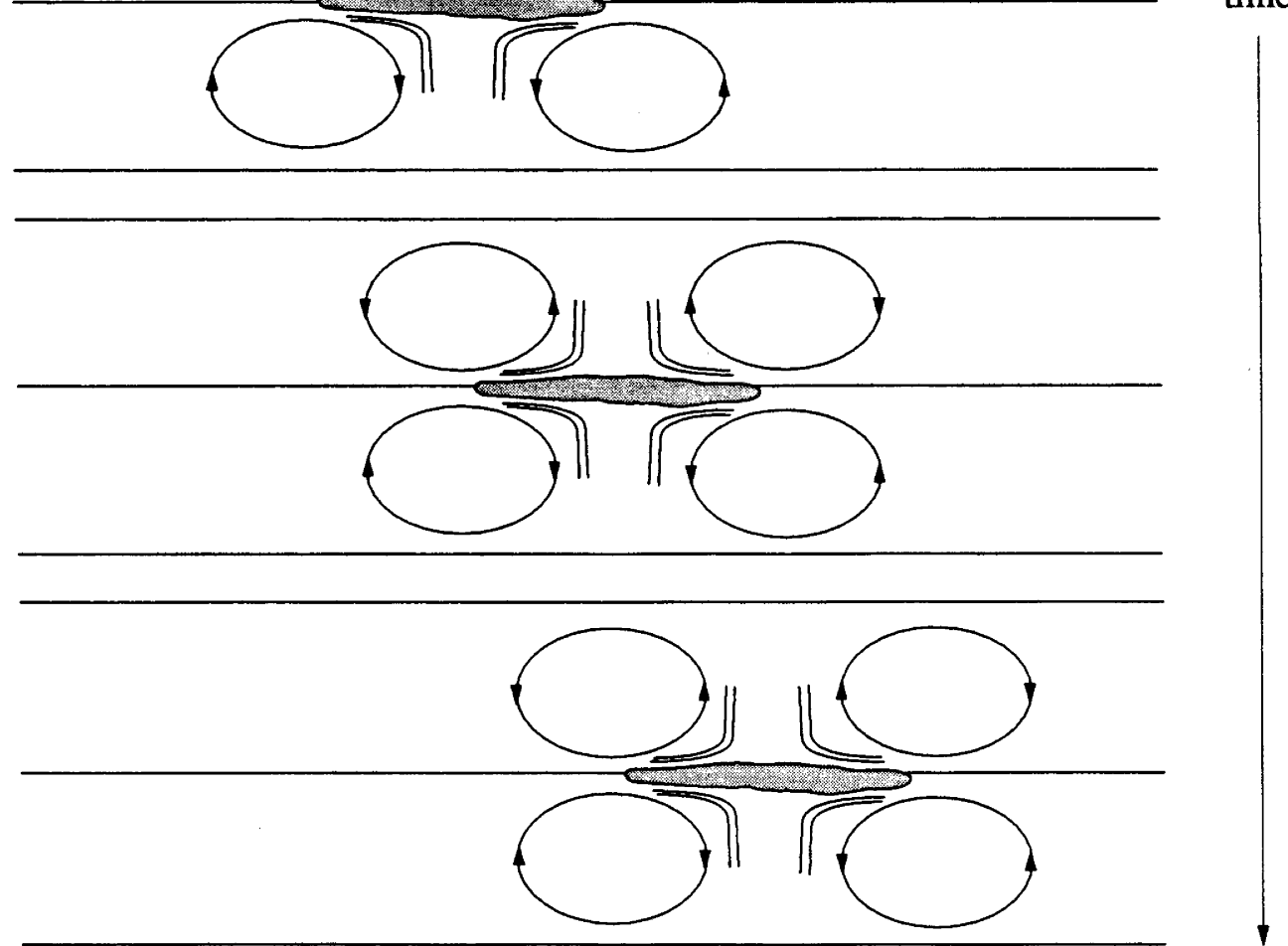

Figure 3.10: Illustration of an overtaking collision. As the two lumps moved to the left, with the leading (left) lump moving slower than the following (right) lump, the size and strength of cells 2 and 3 decreased with time, which hindered the progress of the leading lump and assisted the progress of the following lump. When the lumps collided they formed a single large thick region. The remaining plumes were then swept into the centre of the lump following which cells 2 and 3 disappeared. This single large lump remained intact and moved to the left or right depending on the strength of the convection cells. 


\subsubsection{Long-term cyclic behaviour}

Once a single large thick region of interface formed, the nature of the flow changed to that shown in figures 3.11 to 3.12 . This wave propagated back-and-forth along the channel modulating the fluxes and organizing the convection into large-scale circulations, which reversed direction quasi-periodically. With the exception of flows in very long channels, changes in layer properties during each cycle were negligible. The behaviour of one such quasi-steady cycle will now be described.

A convenient point at which to begin the description is shown in the top frame of each figure. The thick region of interface propagated to the right, while intense plumes at the nose (leading edge) drove two large-scale counter-rotating convection cells in each layer. Marginally unstable boundary layer fluid was produced throughout the thin interface ahead of the wave, however advection by the cells concentrated plumes at the nose. Meanwhile, diffusion re-established the double boundary layer structure of the thin interface left in the wave's wake. The wave propagation was assisted by the cells along its length but hindered by the cells ahead.

The convection evolved in response to the propagation of the wave. As the nose approached the endwall, the size and strength of the cells along the wave increased at the expense of the cells ahead of the wave. In turn, this change in the convection tended to accelerate the wave, particularly away from the sidewalls where the convection was strongest. Thus the leading and trailing edges of the wave were a $\cup$ shape, instead of the purely two-dimensional structures associated with gravity current intrusions and internal solitary waves in the absence of ambient motions.

In general, the wave extended along half the channel as a long thin tail attached to a short thick nose (figure 3.14). The tail was about $2 \mathrm{~mm}$ thick and looked like a continuous stream of well-mixed fluid, whereas the nose was about $4 \mathrm{~mm}$ thick and $10 \mathrm{~mm}$ long and looked like a lump of well-mixed fluid. However, the profile and speed of the wave fluctuated in response to changes in the convection, and occasionally the nose appeared as a wedge-like extension of the tail.

When the wave collided with the endwall the plumes at the nose were cut off and the cells ahead of the wave disappeared, leaving a single tank-scale cell in each layer. These cells continued to sweep both the wave and any plumes developing along the interface against the endwall. This sweeping created a symmetric wedge of interfacial fluid which continued to pile up against the endwall until it extended over only about one-third the channel length. Meanwhile, a bulge corresponding to the reflected nose propagated back through the tail.

Eventually a distinct lump of well-mixed fluid appeared at the left end of the thick region of interface and propagated slowly to the left. The origin of this feature varied; in short channels its appearance coincided with the emergence of the reflected nose, whereas in long channels it appeared to be a new feature. Nevertheless, in both cases the nose propagated along the interface, making greatest progress at the sidewalls where the opposing convection was weakest. At the same time the interfacial fluid trapped against the endwall collapsed under gravity. The nose became more lumplike, and in response the plume activity at the nose increased, which weakened the large-scale circulation behind the nose. This change in the convection allowed more of the trapped interfacial fluid to escape and accelerate away from the endwall.

By the time the nose reached the centre of the channel the plumes at the nose had destroyed the tank-scale cells, and re-established two large-scale counter-rotating cells in each layer. This flow swept fluid away from the endwall and when the nose was just past the centre of the channel the tail detached from the endwall. The cycle then repeated. 
Figures 3.11-3.12: Quasi-steady cycle. The wave propagates to the right while intense plumes at the nose drive two large-scale counter-rotating convection cells in each layer. (b) When the wave collides with the endwall, the plumes at the nose are cut off leaving a single tank-scale cell in each layer. These cells sweep the interfacial fluid against the endwall, but a bulge corresponding to the reflected nose propagates back through the tail. (c) Eventually, the nose appears at the left end of the wave and as it progresses the interfacial fluid trapped against the endwall collapses under gravity and the plume activity at the nose increases. (d) On reaching the centre of the channel, the plumes at the nose have destroyed the tank-scale cells and re-established two counter-rotating cells in each layer. (e) Moments later the tail detaches from the endwall. The cycle then repeats. 
front view

top view
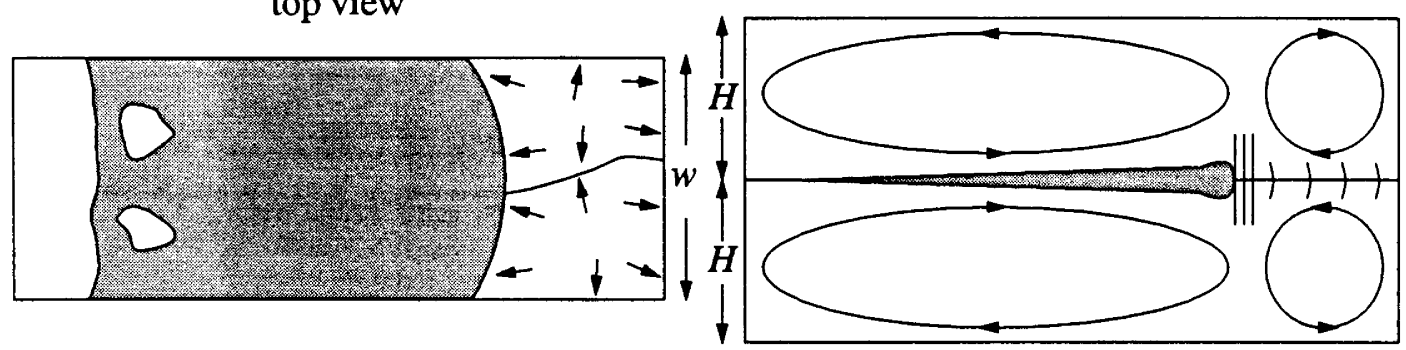

(a)
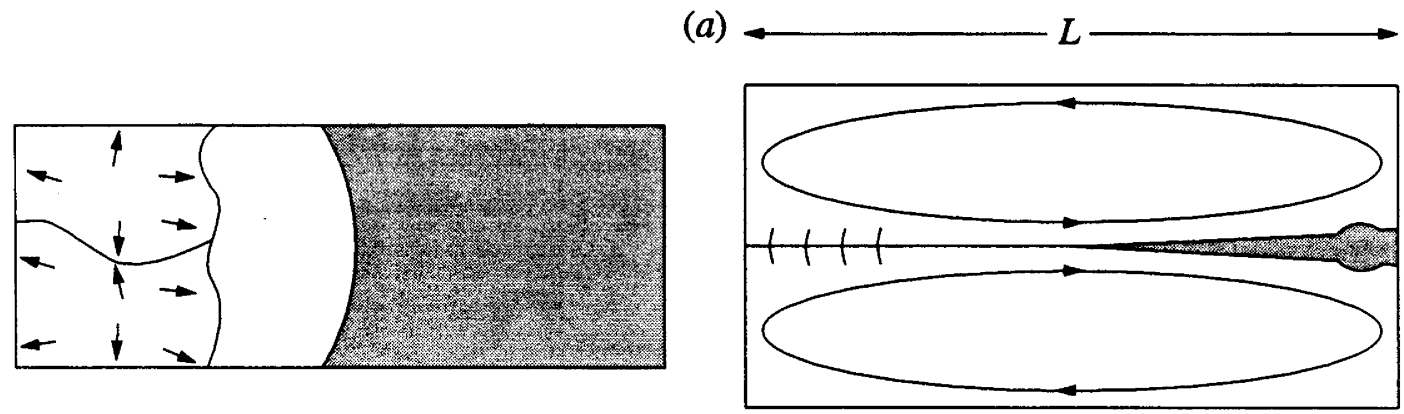

(b)
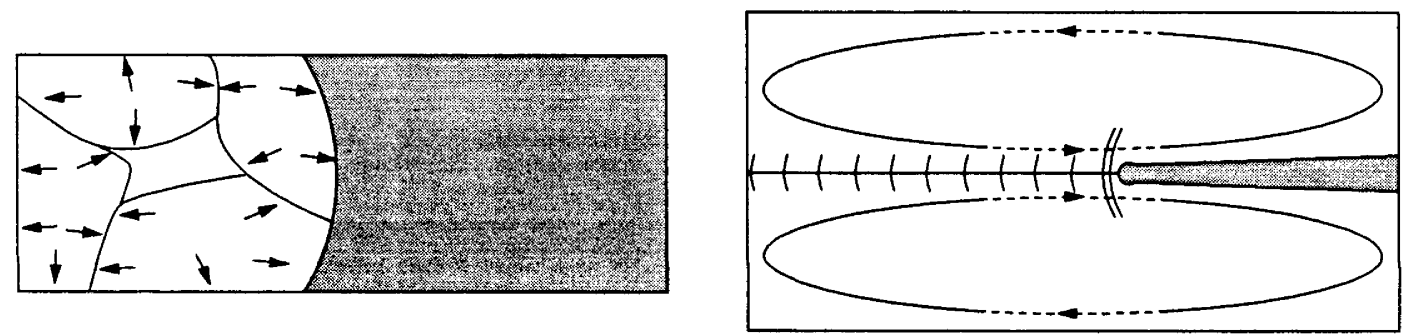

(c)
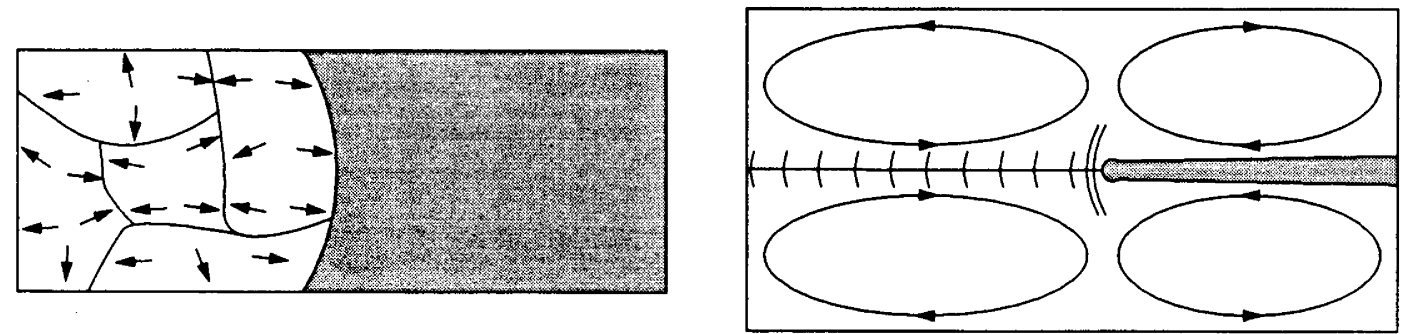

(d)
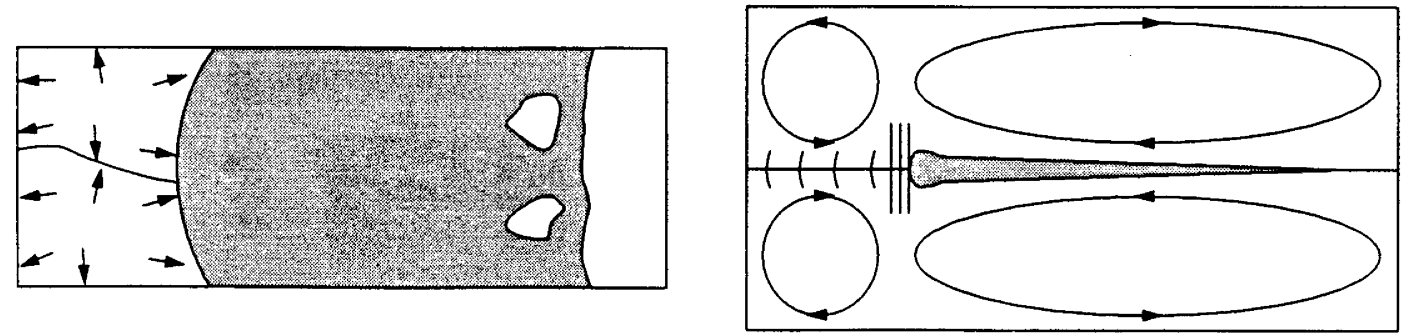

(e)

Figure 3.11: Mlustration of a quasi-steady cycle. 


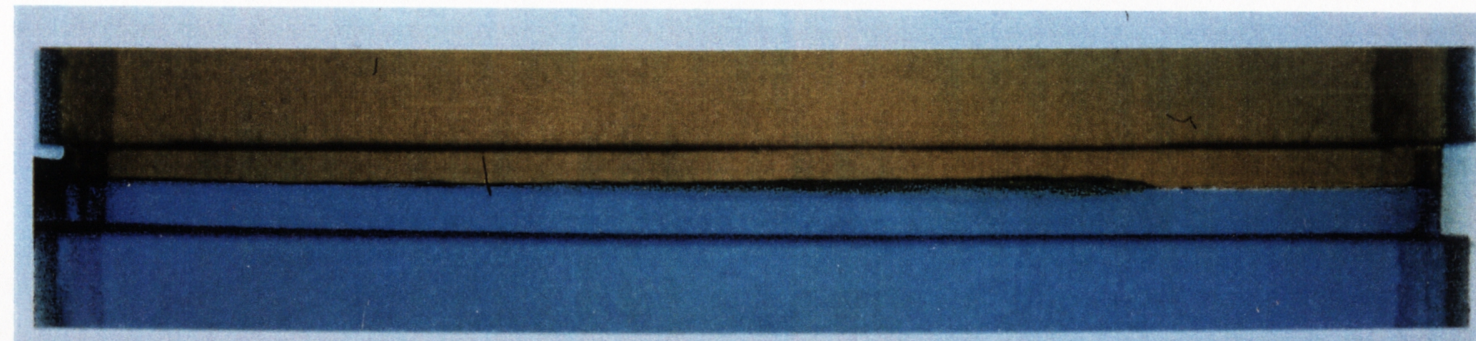

\section{(a)}

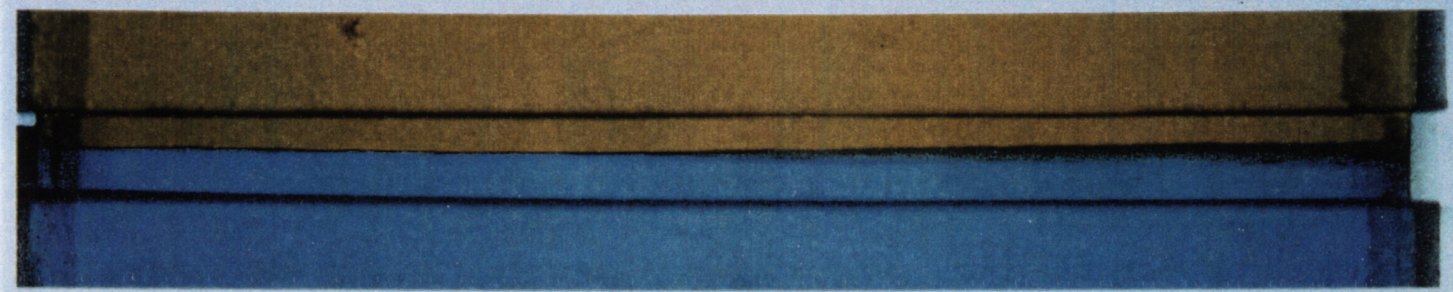

(b)

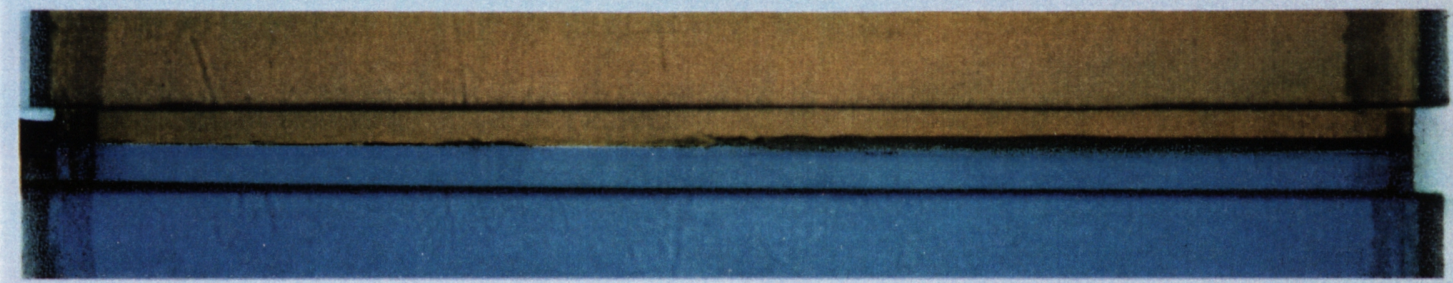

(c)

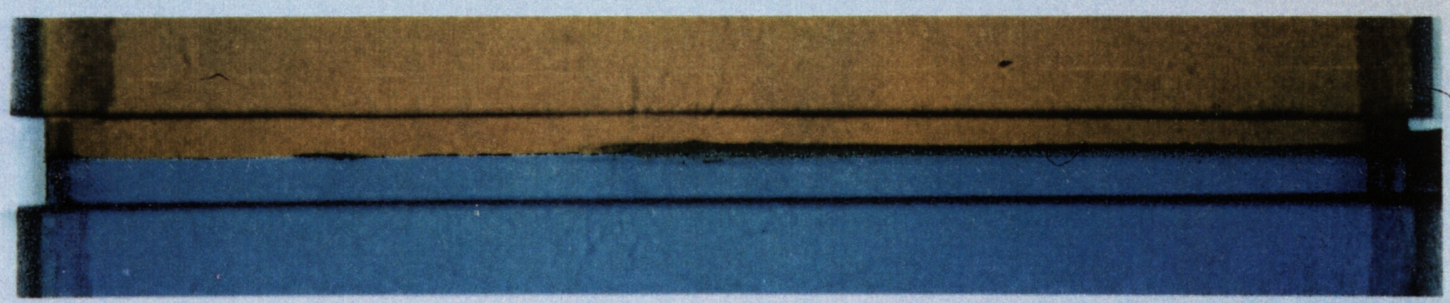

(d)

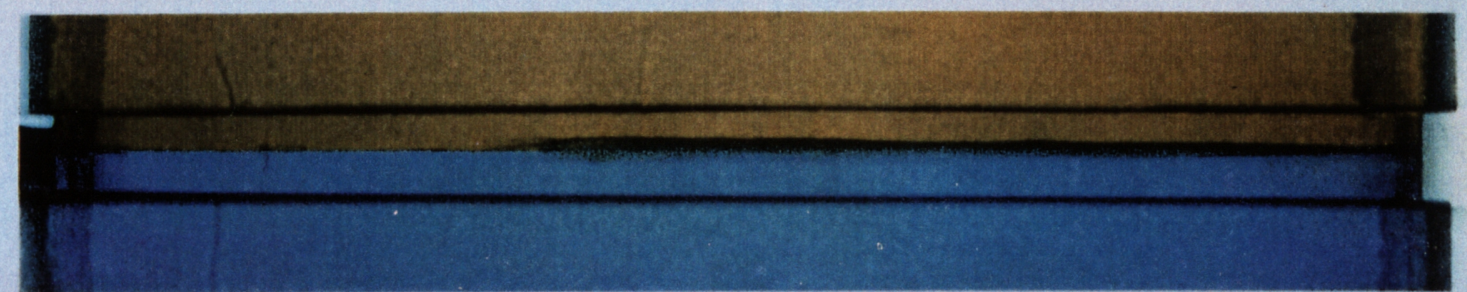

(e)

Figure 3.12: Direct photographs of a quasi-steady cycle. 


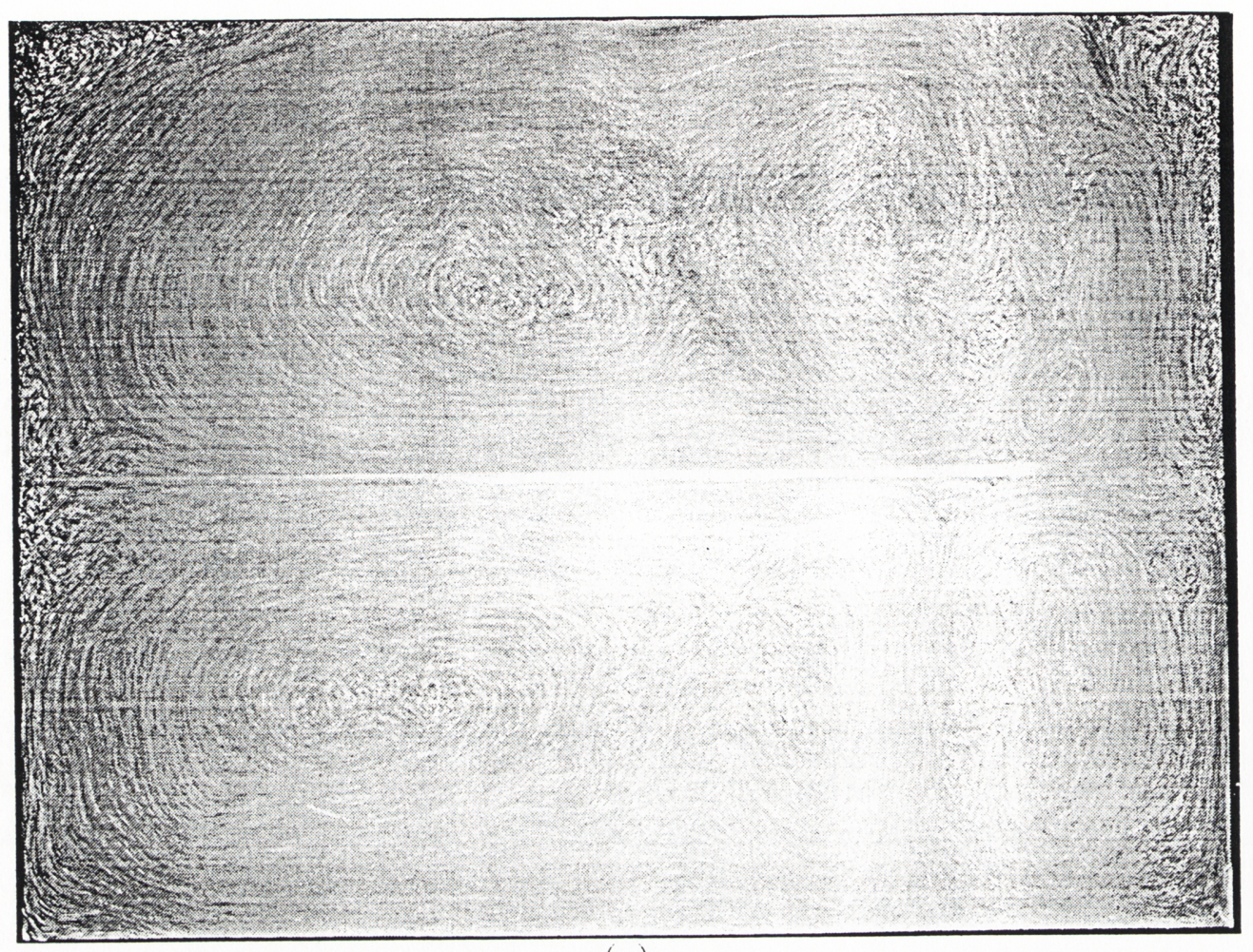

(a)

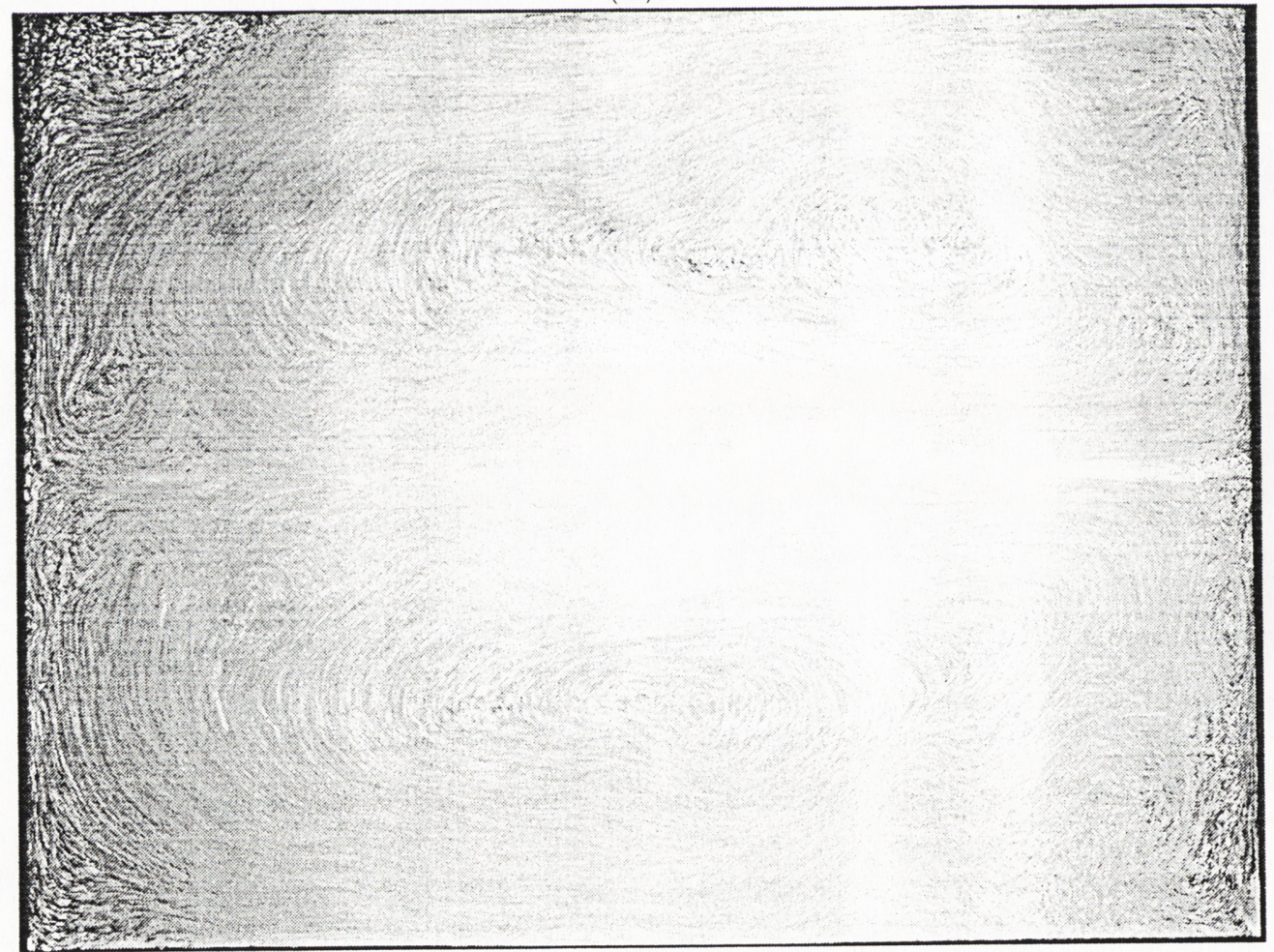

(b)

Figure 3.13: Streak photographs of a quasi-steady cycle. 


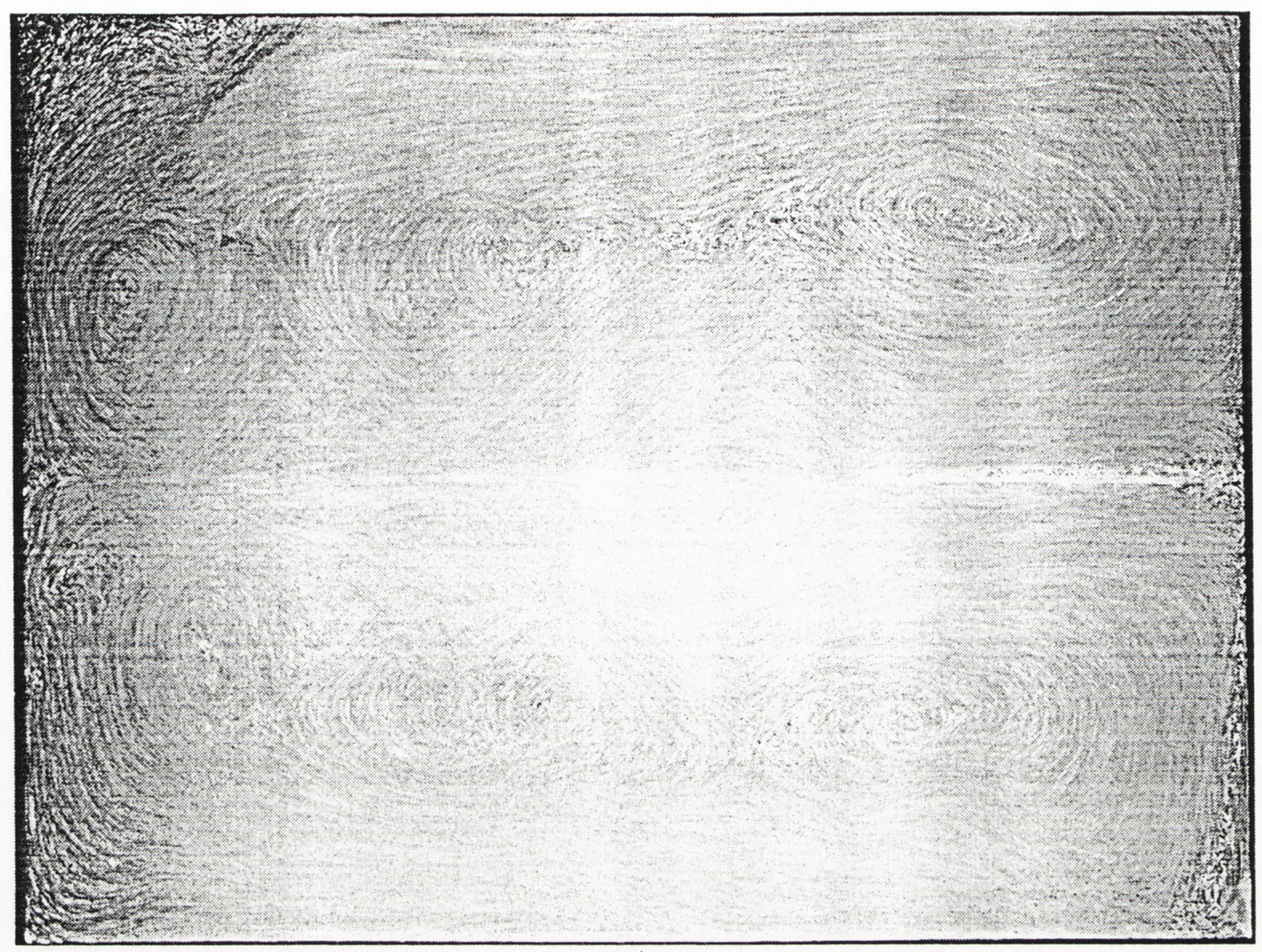

(c)

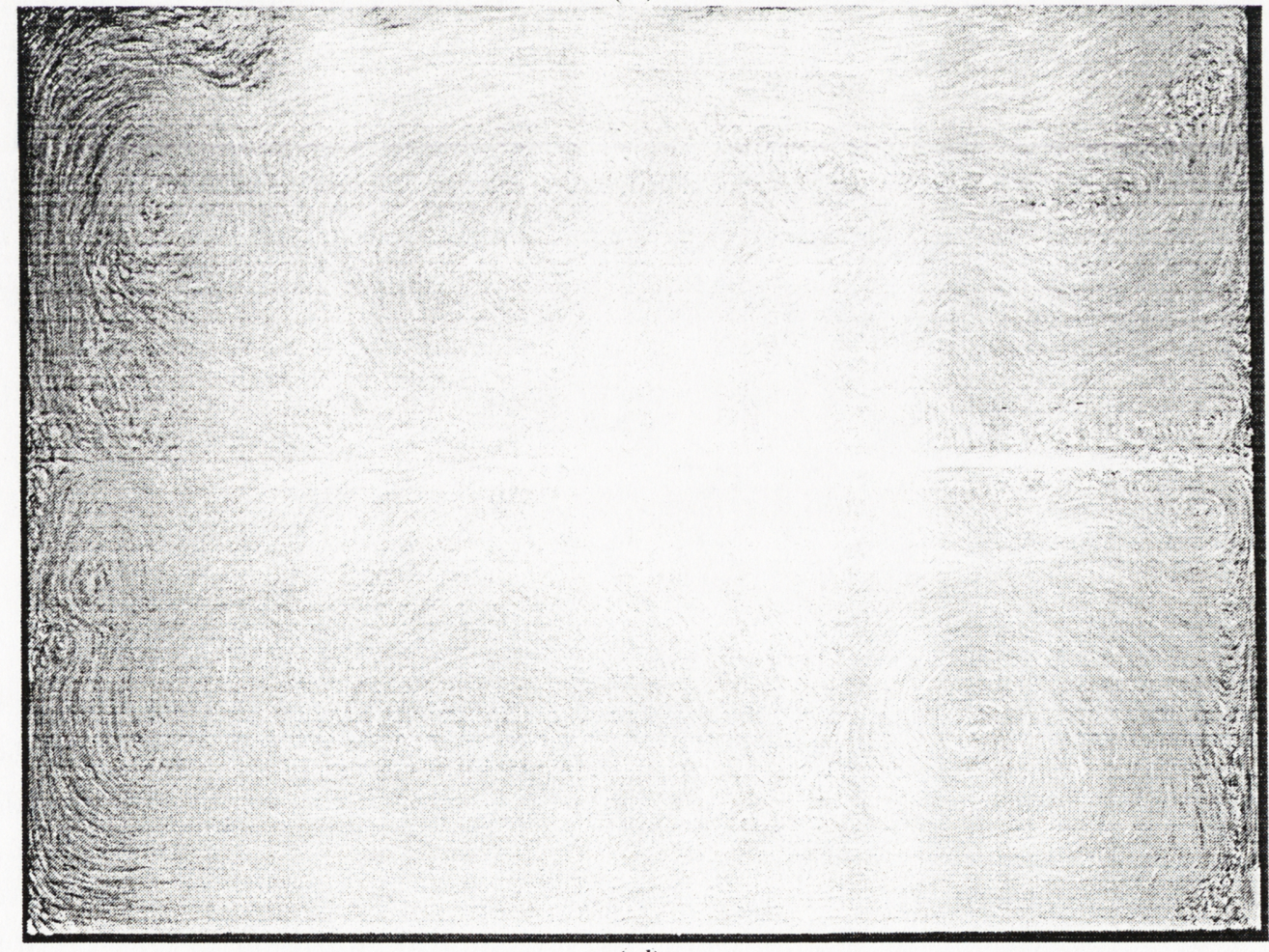

(d)

Figure 3.13 continued. Streak photographs of a quasi-steady cycle. 


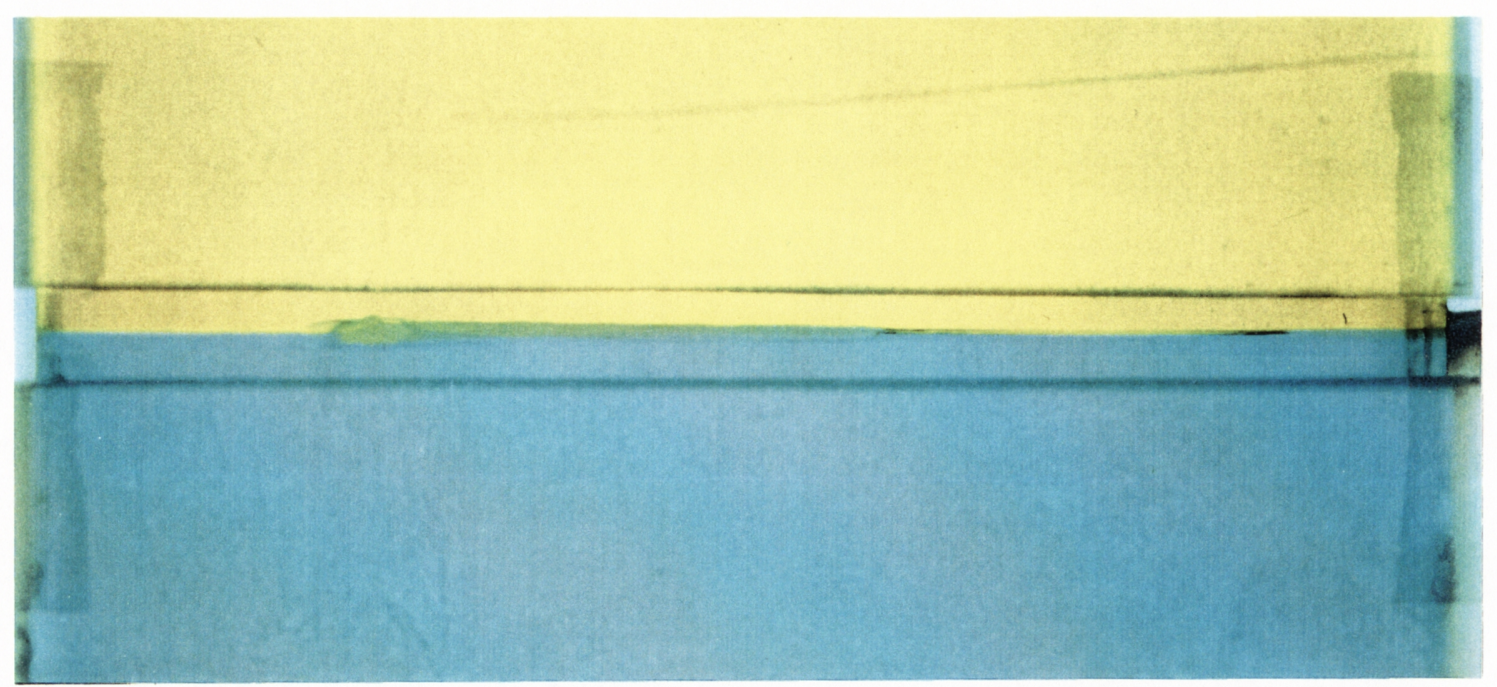

Figure 3.14: Wave profile. The profile and speed of the wave varied on a short time-scale associated with complex coupling dynamics under instantaneous conditions and a long time-scale associated with run-down. However, in general the wave extended along half the channel as a long thin tail attached to a short thick nose.

\subsubsection{Run-down behaviour}

This cyclic flow continued for about 4 hours, during which time there was a two-fold decrease in $\alpha \Delta T$ and $R_{\rho}$ increased from 1.02 to 1.40 (table 3.1). However, whilst the qualitative behaviour of the flow remained unchanged, several quantitative aspects changed: the thickness of the thin region of interface increased, the length of the thick region of interface increased, the buoyancy fluxes and convective velocities decreased and the wavespeed decreased. At the end of this time the characteristic thick and thin regions of the interface were no longer visible, but the interface continued to slowly seiche back-and-forth.

\subsection{Experimental plan}

Once the experimental procedure was refined and the general nature of the flow determined, two sets of experiments were carried out for which detailed observations and measurements were made of the flow. The first ten experiments were designed to ascertain the dependence on $\alpha \Delta T$ and $R_{\rho}$, and to this end were carried out in the $15 \times 5 \mathrm{~cm}$ channel and started with the widest possible range of solute concentrations (table 3.1). Following this, six experiments, each started with the same solute concentrations, were carried out in channels with horizontal cross-sections ranging from $L \times w=15 \times 5$ to $180 \times 15 \mathrm{~cm}$ in order to ascertain the dependence on the channel aspect ratio $L / H$ (table 3.2 ).

The experimental results are presented in the following two sections, with, for clarity, $\S 3.6$ and 3.7 focusing on the wave-convection coupling and interfacial fluxes respectively. Except where stated, the results presented are those for the first set of experiments, that is, for the experiments carried out in the $15 \times 5 \mathrm{~cm}$ channel. 


\begin{tabular}{|c|c|c|c|c|c|c|c|c|c|}
\hline \multirow[b]{2}{*}{ run } & \multirow[b]{2}{*}{ symbol } & \multicolumn{2}{|c|}{ initial conditions } & \multicolumn{3}{|c|}{ onset of coupling } & \multicolumn{3}{|c|}{ final measurements } \\
\hline & & $\bar{\alpha} \Delta T$ & $R_{\rho}$ & $t(\mathrm{~h}: \mathrm{m}: \mathrm{s})$ & $\bar{\alpha} \Delta T$ & $R_{\rho}$ & $t(\mathrm{~h}: \mathrm{m}: \mathrm{s})$ & $\bar{\alpha} \Delta T$ & $R_{\rho}$ \\
\hline 1.1 & $\diamond$ & 0.0279 & 1.05 & 1:01:58 & 0.0216 & 1.16 & $4: 36: 54$ & 0.0165 & 1.32 \\
\hline 1.2 & $\triangle$ & 0.0348 & 1.04 & 1:04:44 & 0.0262 & 1.16 & $4: 21: 09$ & 0.0205 & 1.32 \\
\hline $1.3 a$ & 0 & 0.0462 & 1.03 & 0:50:58 & 0.0356 & 1.14 & $4: 49: 46$ & 0.0258 & 1.34 \\
\hline $1.3 b$ & & 0.0383 & 1.10 & 0:28:10 & 0.0345 & 1.15 & $3: 51: 24$ & 0.0257 & 1.33 \\
\hline $1.3 c$ & & 0.0321 & 1.19 & $0: 10: 20$ & 0.0316 & 1.21 & $1: 52: 06$ & 0.0279 & 1.30 \\
\hline 1.4 & $\square$ & 0.0543 & 1.02 & 0:53:18 & 0.0404 & 1.14 & $4: 28: 06$ & 0.0294 & 1.33 \\
\hline 1.5 & $\nabla$ & 0.0688 & 1.02 & 0:50:06 & 0.0512 & 1.14 & 4:03:21 & 0.0391 & 1.30 \\
\hline $1.6 a^{\dagger}$ & $x$ & 0.0975 & 1.02 & $0: 50: 18$ & 0.0706 & 1.16 & $4: 02: 36$ & & \\
\hline $1.6 b^{\dagger \dagger}$ & + & 0.0975 & 1.02 & 0:55:06 & 0.0714 & 1.15 & 4:01:52 & & \\
\hline 1.7 & $\odot$ & 0.1256 & $\begin{array}{c}1.02 \\
\text { ffina } \\
\text { tlayer }\end{array}$ & $\begin{array}{l}1: 00: 04 \\
\text { mples co } \\
\text { lded in } r\end{array}$ & $\begin{array}{c}0.0918 \\
\text { aminate } \\
\text { verse ord }\end{array}$ & 1.14 & $4: 24: 30$ & 0.0703 & 1.30 \\
\hline
\end{tabular}

Table 3.1: Experimental parameters and results for the first set of experiments. This set was designed to ascertain the dependence of the flow on $\alpha \Delta T$ and $R_{\rho}$, and to this end experiments were carried out in the $15 \times 5 \mathrm{~cm}$ channel and started with different solute concentrations. Experiments 1.1-1.3a,1.4-1.7 were started with the widest possible range of $\alpha \Delta T$ values while maintaining $R_{\rho}$ near unity. This range was limited by the practical difficulties associated with creating an interface between layers of almost equal density and the finite solubility of salt. Experiments $1.3 b, c$ were started with larger $R_{\rho}$ and with $\alpha \Delta T$ values such that they evolved along the same $\left(\alpha \Delta T, R_{\rho}\right)$ curve as experiment $1.3 a$. For each experiment the time at which a single wave formed was noted, and the corresponding values of the independent parameters inferred from the measured values at nearby times. The measurements indicate that wave-convection coupling occurred once the density-anomaly ratio exceeded the critical value $R_{\rho}^{\mathrm{c}}=1.15$, irrespective of the elapsed time (e.g. experiment $1.3 b$ ), and when flows were started above this critical value coupling started immediately (e.g. experiment $1.3 c$ ).

\begin{tabular}{cccccccccc} 
& & & \multicolumn{4}{c}{ onset of coupling } & \multicolumn{3}{c}{ final measurements } \\
run & symbol & $L \times w$ & $L / H$ & $t(\mathrm{~h}: \mathrm{m}: \mathrm{s})$ & $\bar{\alpha} \Delta T$ & $R_{\rho}$ & $t(\mathrm{~h}: \mathrm{m}: \mathrm{s})$ & $\bar{\alpha} \Delta T$ & $R_{\rho}$ \\
2.1 & $\square$ & $15 \times 5$ & 1.3 & $0: 53: 18$ & 0.0404 & 1.14 & $4: 28: 06$ & 0.0294 & 1.33 \\
2.2 & $\triangle$ & $30 \times 5$ & 2.6 & $0: 52: 59$ & 0.0399 & 1.13 & $5: 18: 54$ & 0.0297 & 1.31 \\
2.3 & $\bigcirc$ & $40 \times 5$ & 3.5 & $1: 08: 10$ & 0.0347 & 1.18 & $4: 29: 03$ & 0.0264 & 1.35 \\
2.4 & $\diamond$ & $75 \times 15$ & 6.5 & $1: 26: 24$ & 0.0340 & 1.20 & $3: 22: 59$ & 0.0289 & 1.29 \\
2.5 & $\nabla$ & $90 \times 15$ & 7.8 & $1: 54: 59$ & 0.0350 & 1.21 & $4: 56: 05$ & 0.0299 & 1.31 \\
2.6 & $\times$ & $180 \times 15$ & 15.6 & $2: 16: 21$ & 0.0348 & 1.22 & $5: 31: 53$ & 0.0298 & 1.32
\end{tabular}

Table 3.2: Experimental parameters and results for the second set of experiments. This set was designed to ascertain the dependence of the flow on the channel aspect ratio, $L / H$, and to this end experiments were carried out in channels with different horizontal crosssections and started with the same solute concentrations; $\bar{\alpha} \Delta T=0.0543$ and $R_{\rho}=1.02$. The flow was the same in short $(15 \mathrm{~cm})$ and long $(180 \mathrm{~cm})$ channels, but, as expected, the critical $R_{\rho}$ for coupling increased as the channel length increased. 


\subsection{Results: wave-convection coupling}

To supplement the qualitative observations presented in $\S 3.3$, measurements were made of the wave and convective motions throughout each experiment. Attention focused on the behaviour when a single wave propagated back-and-forth along the channel, organizing the convection into large-scale circulations.

\subsubsection{Onset of wave-convection coupling}

For each experiment the time at which a single wave formed was noted, and the corresponding values of the independent and dependent parameters inferred from the measured values at nearby times. Figure 3.15 shows the evolution of $\alpha \Delta T$ and $R_{\rho}$ for the experiments, with the conditions at the onset of coupling in bold. The measurements indicate that for coupling to occur the density-anomaly ratio must exceed the critical value

$$
R_{\rho}^{\mathrm{c}}=1.15 \pm 0.01 \quad \text { for } \quad 0.0279 \leq(\bar{\alpha} \Delta T)_{0} \leq 0.1256,
$$

irrespective of the elapsed time (e.g. experiment 1.3b), and when flows were started above this critical value coupling started immediately (e.g. experiment $1.3 \mathrm{c}$ ); table 3.1 .

A physical argument supporting the concept of a critical density-anomaly ratio for the onset of coupling can be made by assuming that the wavespeed and the horizontal convective velocities must be similar for effective coupling. At the start of an experiment the buoyancy flux and resulting convective velocities are large because the interface is thin. Consequently, the wave amplitudes and speeds are too small to couple with the convective flow. However, as the system runs-down the interface thickens, thereby supporting larger, and faster, waves which cause horizontal variations in the buoyancy flux large enough to organize the convection into large-scale circulations. At the same time the buoyancy flux and convective velocities decrease. Eventually the wavespeeds are fast enough and the convective velocities are slow enough for coupling to occur. Since the wave amplitude and convective velocities are both controlled by the interface thickness, and this is a strong function of $R_{\rho}$, it is expected that a critical value of $R_{\rho}$ must be exceeded before coupling can occur.

\subsubsection{Definitions and measurements}

The long-term flow consisted of a single wave which propagated back-and-forth along the channel, organizing the convection in each layer into two cells which oscillated in length (figure 3.11). A complete description of this flow required measurements be made several times during each cycle. This was not possible however, as all measurements had to be made in real time because video images were unable to resolve variations in the interface thickness. Consequently, the quantitative investigation focused on the long-term behaviour of the flow, with measurements made once during each cycle.

In all experiments the times at which the wave (nose) reached the endwalls were noted, and, for flows carried out in long channels, position-time measurements were made while the wave propagated along the channel. Although the three-dimensional structure of the wave complicated measurements, the time at which the wave reached a given position was measured to within \pm 2.5 seconds at low $R_{\rho}$ and \pm 5 seconds 


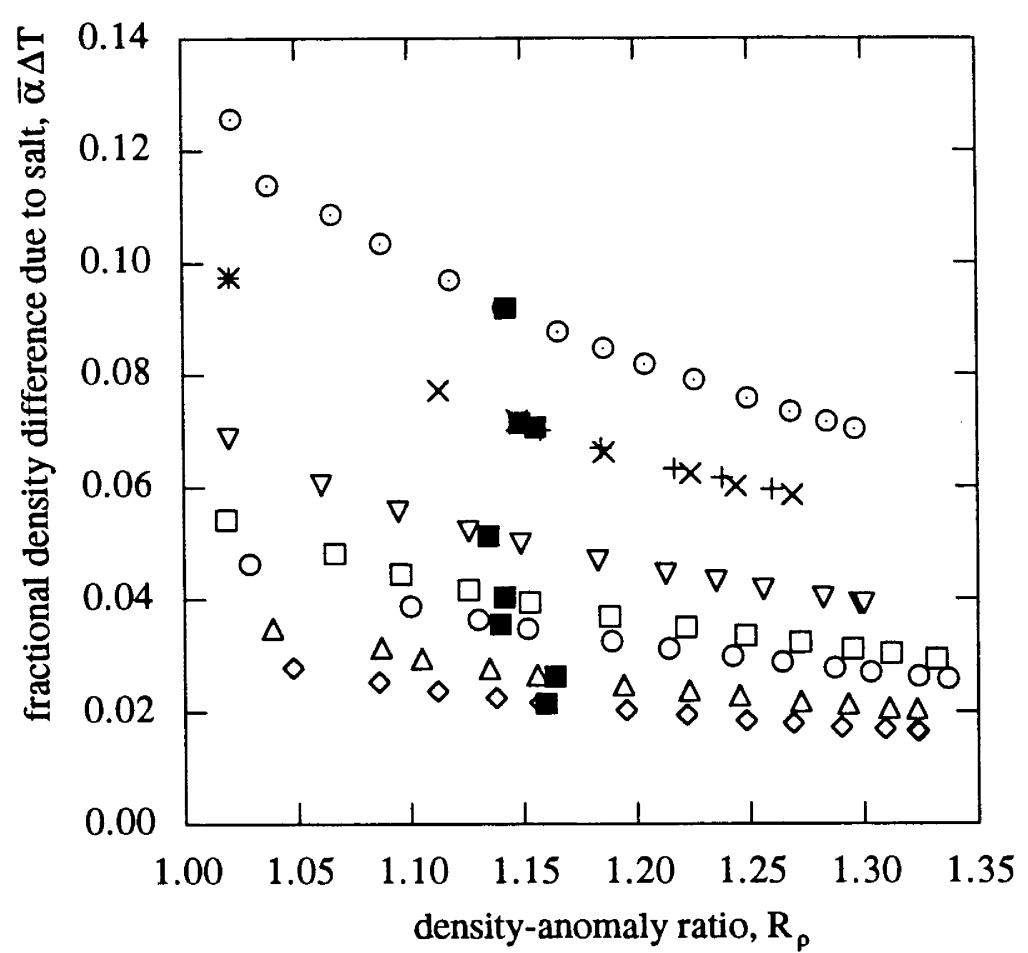

(a)

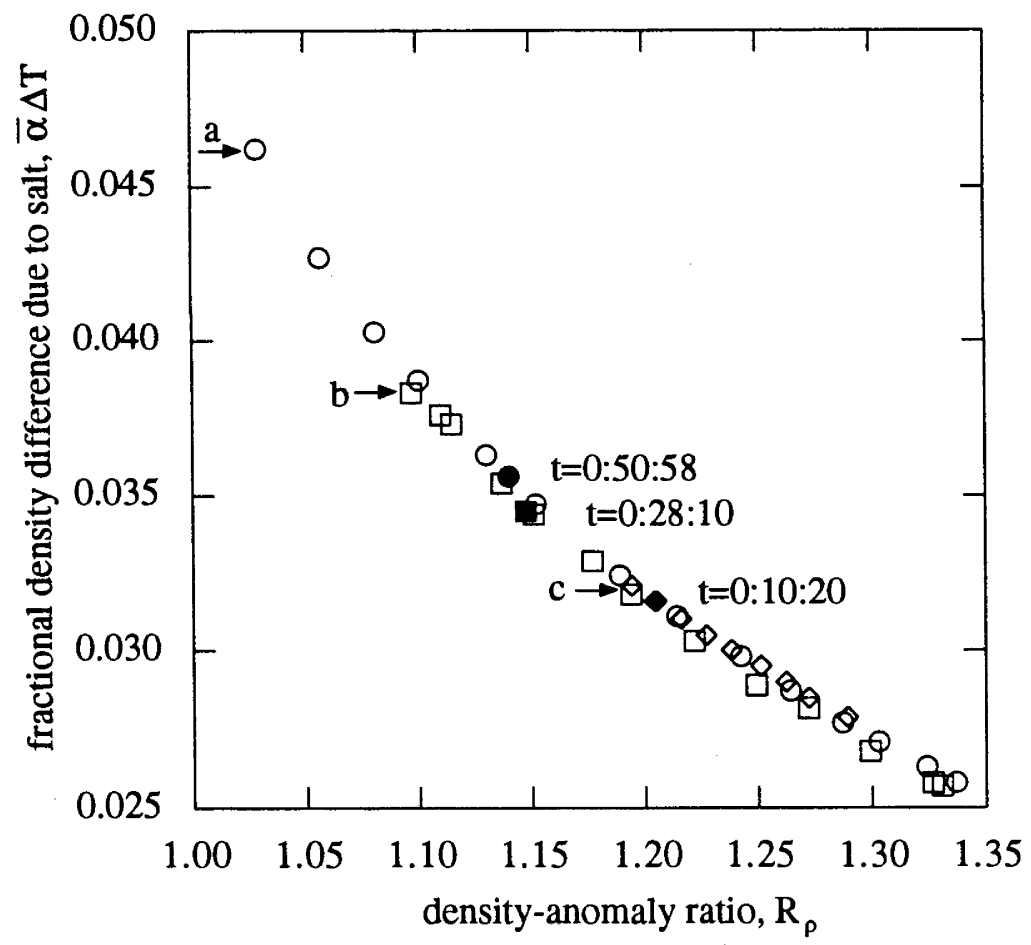

(b)

Figure 3.15: $\bar{\alpha} \Delta T$ vs. $R_{\rho}$ for the first set of experiments. (a) Experiments 1.1-1.3a,1.41.7 and $(b) 1.3 a(\bigcirc), 1.3 b(\square)$ and $1.3 c(\diamond)$, with the conditions at the onset of coupling in bold. During each experiment the concentration differences across the interface decreased in response to the interfacial fluxes. With $T$ being the more diffusive component $\alpha \Delta T$ decreased faster than $\beta \Delta S$, and therefore $R_{\rho} \equiv \beta \Delta S / \alpha \Delta T$ increased. The measurements indicate that coupling occurred once the density-anomaly ratio exceeded the critical value $R_{\rho}^{c}=1.15$, irrespective of the elapsed time (e.g .experiment $1.3 b$ ), and when experiments were started above this critical value coupling started immediately (e.g. experiment 1.3c). This behaviour occurred because the wave amplitude and convective velocities were both controlled by the interface thickness, and this was a strong function of $R_{\rho}$. 
at high $R_{\rho}$. However, it was difficult to measure the nose position while the wave was banked up against an endwall, and thus the position-time measurements were generally restricted to the remaining two-thirds of the cycle.

The waves were also characterized by the thickness of the thin interface $(2 h$, i.e. the undisturbed depth of the stratification), the thickness of the thick interface $\left(2 h_{*}\right.$, i.e. the wave amplitude $\left.a=h_{*}-h\right)$ and the length of the thick region $(\lambda$, i.e. the wavelength). Measurements of these length scales were not possible at high $R_{\rho}$ because the thick and thin regions of the interface became less distinct during run-down. In addition, at low $R_{\rho}$ the thin interface was observed to be $<1 \mathrm{~mm}$ thick, but neither direct visualization nor conductivity profiles could resolve the exact thickness. Thus measurements were restricted to the thickness and length of the thick interface, which, for convenience, were measured when the wave was fully banked-up against the endwalls. Although these measurements provided useful information, without knowing the thickness of the thin interface ahead of the wave, it was not possible to determine the non-dimensional wave amplitude, $a / h=\left(h_{*}\right.$ $h) / h$, and thus comparing the observed waves with gravity current intrusions and internal solitary waves was difficult.

Several unsuccessful attempts were made at determining the convective velocities: the large refractive index variations negated laser doppler anemometry, the flow velocities were too small for hot-wires and the layer densities changed too quickly for particle tracking. Indeed, had these techniques worked, it would still remain to distinguish the long time-scale motions associated with the large-scale circulations from the short time-scale motions associated with the plumes. The fact that plumes, which had a different composition to their surroundings, were swept along the interface by the large-scale circulations, introduced the possibility of direct measurement of the horizontal velocities. However, comparing signals from conductivity probes placed next to each other in the flow did not produce reliable velocity estimates, and a similar technique using light diodes was equally unsuccessful. Whilst comprehensive measurements of the convective velocities could not be made, the distortion of dye lines and streak photographs indicated that the horizontal velocities were $O\left(1-2 \mathrm{~mm} \mathrm{~s}^{-1}\right)$.

\subsubsection{Wavespeed}

An important property of the waves is their speed of propagation. Figure 3.16 shows the time taken by the wave to travel the channel length (i.e. the half-period of the wavemotion, $\theta$ ) for the duration of one experiment. The wave persisted for about 4 hours and travelled $20 \mathrm{~m}$, whereas similar waves without convection only persisted for 2 minutes and travelled $3 \mathrm{~m}$ (Chapter 2). In addition, the half-period did not increase monotonically, but instead, while the average time taken by the wave to travel the channel length increased from 95 to 115 seconds, there were large ( \pm 15 seconds) deviations from average values. This behaviour indicates that the wave gained energy from the convection, and, while the decrease in convective activity produced a long time-scale decrease in wavespeed, the complex wave-convection coupling dynamics produced short time-scale variations.

In all experiments the short time-scale variations appeared random. However, because measurements were made at irregular intervals, the standard techniques for analysis of time-series data could not be used, and thus it was not possible to ascertain whether these variations resulted from random noise or deterministic chaos. Nevertheless, two factors which may have caused the variations were identified. 


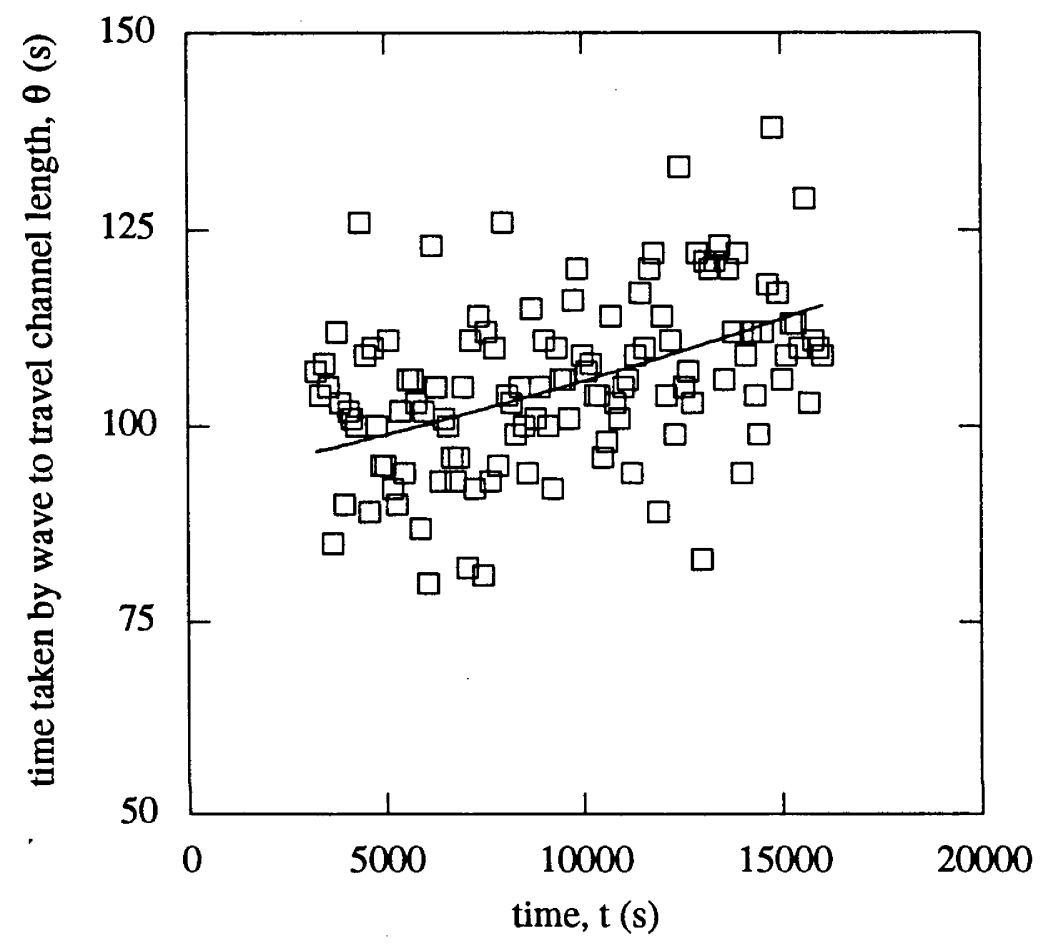

Figure 3.16: Half-period of wavemotion $v s$. time since the start of the experiment. The wave persisted for about 4 hours and travelled $20 \mathrm{~m}$, with the average time taken to travel the channel length (— $(-)$ increasing from 95 to 115 seconds as the convection weakened. However, the half-period did not increase monotonically, but instead the complex wave-convection coupling dynamics produced large ( \pm 15 seconds) fluctuations $(\square)$. For each experiment the average wavespeeds were determined from the derivative of the second degree polynomial that best fits the displacement-time measurements, $\mathrm{d} X(t) / \mathrm{d} t$ where $X(t)=A_{0}+A_{1} t+A_{2} t^{2}$. This procedure gave wavespeeds that increased linearly with time, but the associated half-period estimates increased as $\theta=L / c=L /\left(A_{1}+2 A_{2} t\right)$, and this explains curvature in the solid line. 
First, small three-dimensional convection cells were observed along the thin interface ahead of the wave, and as these cells moved randomly about, expanding, contracting and merging, the conditions experienced by the wave changed. Second, small thick regions of interface occasionally formed along the otherwise thin interface ahead of the wave, and the wave may have suffered a phase shift on colliding with these lumps.

To determine the dependence of the wavespeed on the layer properties and channel dimensions, the long time-scale behaviour had to be distinguished from the short time-scale variations. For each experiment the 'time-dependent average wavespeeds' were calculated by taking the derivative of the second degree polynomial that best fits the displacement-time measurements, i.e. $\mathrm{d} X(t) / \mathrm{d} t$ where $X(t)=A_{0}+A_{1} t+A_{2} t^{2}$. This procedure produced an analytic expression for the wavespeed, which allowed values to be conveniently calculated at times when the independent parameters were known, and the resulting estimates agreed with difference estimates (figure 3.16).

Figure 3.17( $a$ ) shows the average wavespeed for each experiment plotted against the density-anomaly ratio. Two features emerge. First, during the lifetime of each wave, $\alpha \Delta T$ decreased two-fold, $R_{\rho}$ increased from 1.15 to 1.40 , and in response the wavespeed decreased by about $20 \%$. Second, each flow occupied a different region of the parameter space, with flows started with larger $\alpha \Delta T$ values having faster waves. Thus the wavespeed was largest when the driving interfacial density difference $(\alpha \Delta T)$ was large and the interface close to static stability $\left(R_{\rho} \rightarrow 1\right)$, that is, when the fluxes were largest. However, whilst there was an order of magnitude difference in the fluxes during experiments 1.1 and 1.7 (i.e. $(\alpha \Delta T)_{0}=0.0279$ and 0.1256 ), the fact that wavespeeds in the latter, more active, flow were only about two times larger than those in experiment 1.1 indicates that the wavespeed was weakly dependent on the flux.

To examine this behaviour in a more rigorous fashion, the argument used to explain the concept of a critical density-anomaly ratio for the onset of coupling was made more quantitative. Consider a flow in which a wave propagates along a channel assisted by a tank-scale convection cell in each layer (figure 3.18). The wave is thick enough to turn off the local interfacial fluxes, and thereby gives rise to tank-scale density gradients which organize the convection over the channel length. Hence the horizontal convective velocities are given by

$$
u \sim\left(g \delta \rho H / \rho_{0}\right)^{\frac{1}{2}}
$$

where $\delta \rho$ is the horizontal density difference along each layer and $\rho_{0}$ is a reference density. In the special, unattainable, case of a steady balance between the interfacial flux and horizontal advection,

$$
F \sim u \delta \rho H / L
$$

where $F$ is the mass flux (i.e. units $\mathrm{gm} \mathrm{cm}^{-2} \mathrm{~s}^{-1}$ ) through the thin interface ahead of the wave. Rearranging gives

$$
u \sim(b L)^{\frac{1}{3}}
$$

where $b=g F / \rho_{0}$ is the equivalent buoyancy flux (i.e. units $\mathrm{cm}^{2} \mathrm{~s}^{-3}$ ). ${ }^{1}$ Thus the usual relationship between the convective velocities and buoyancy flux (i.e. $u \sim b^{\frac{1}{3}}$

\footnotetext{
${ }^{1}$ This result can also be derived by assuming that all the potential energy associated with the unstable boundary layers is converted into kinetic energy in the convecting layers. However, this approach is less convincing because the thickness and density anomaly of the boundary layers must be estimated, and these quantities are not known with any certainty.
} 


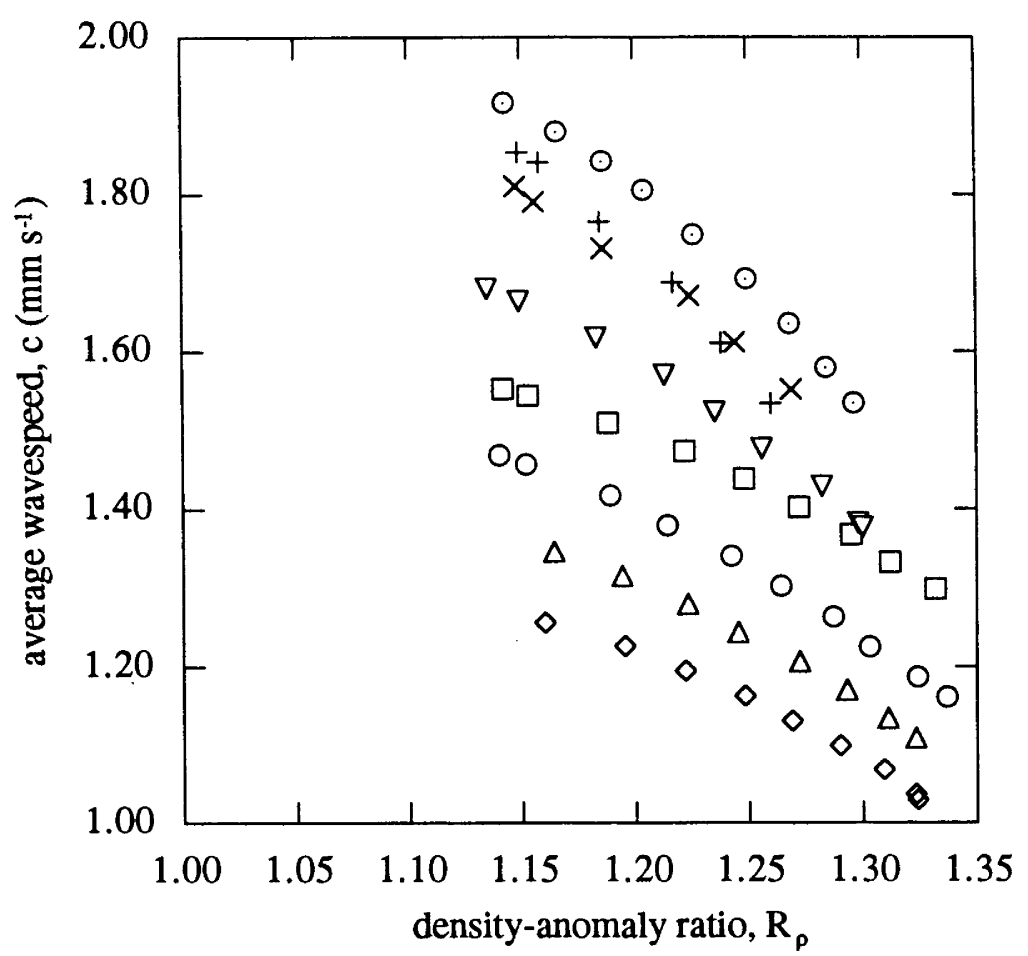

(a)

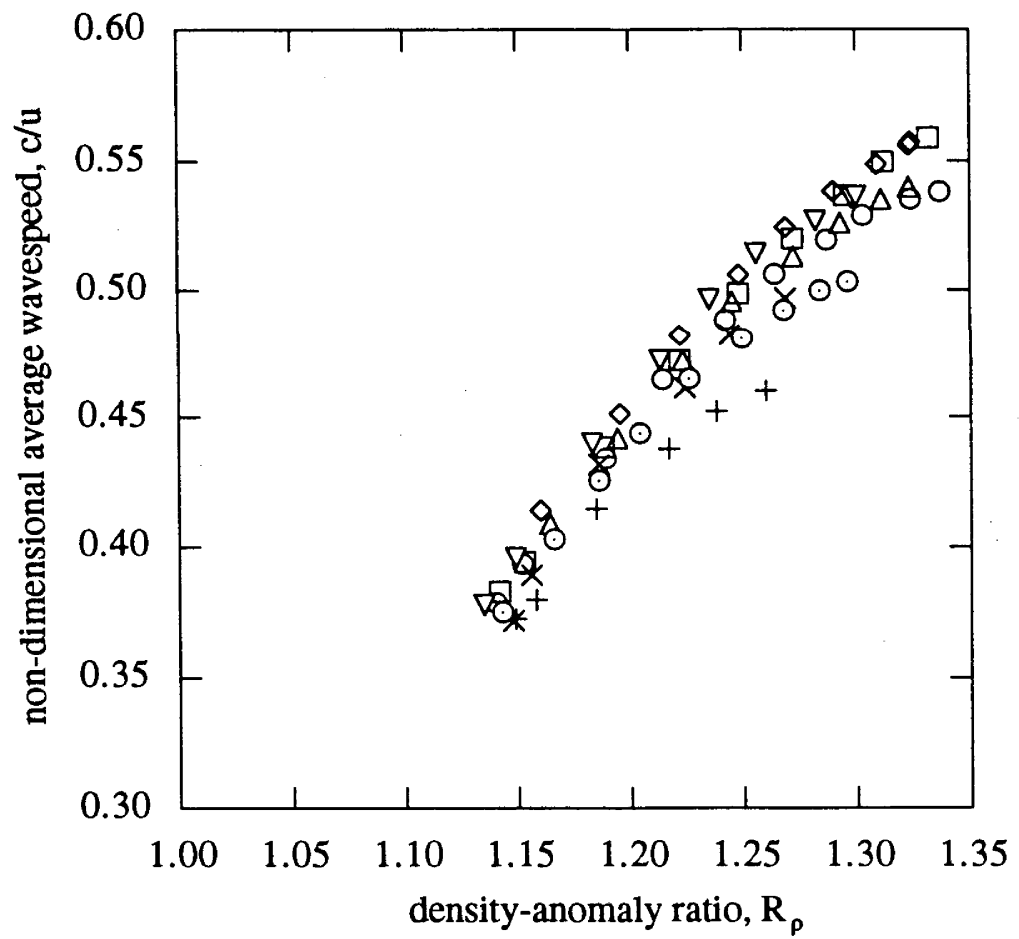

(b)

Figure 3.17: Wavespeed results for the first set of experiments. (a) c vs. $R_{\rho}$. During the lifetime of each wave, $\alpha \Delta T$ decreased two-fold, $R_{\rho}$ increased from 1.15 to 1.40 , and in response the wavespeed decreased by about $20 \%$. In addition, the flows which were started with larger $\alpha \Delta T$ values had faster waves. Thus the wavespeed was largest when the driving interfacial density difference $(\alpha \Delta T)$ was large and the interface close to static stability $\left(R_{\rho} \rightarrow 1\right)$. (b) $c / u$ vs. $R_{\rho}$. A steady-state model suggests that the horizontal convective velocities are proportional to $(B L)^{\frac{1}{3}}$ once the convection is organized over the channel length. Non-dimensionalizing the wavespeeds for each experiment by this velocity collapses all the measurements onto the same curve, and this confirms that coupling requires a matching of the wave and convection speeds. The increase in $c / u$ with increasing $R_{\rho}$ suggests that the exact nature of the coupling depends on the structure of the interface. 


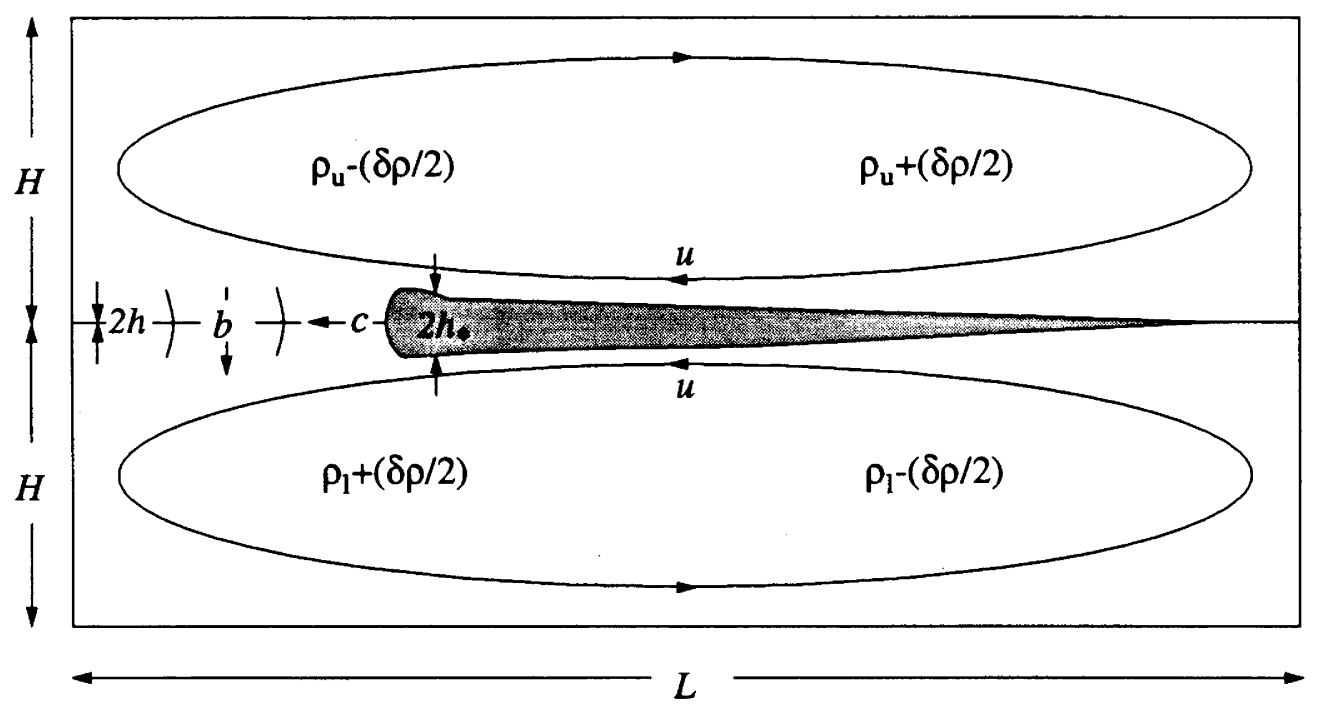

Figure 3.18: Wave-convection coupling model. A wave propagates along a channel, with the convection organized over the channel length by the tank-scale density gradients resulting from the horizontal variations in the interfacial buoyancy flux. The model assumes that the wave selects an amplitude such that its speed is similar to the horizontal convective velocities, i.e. $c \sim u$. Analytical results are derived for the case of a steady balance between the interfacial flux and horizontal advection, and found to agree with the experimental measurements.

Hunt (1984)) applies, despite the fact that the convection is organized over the channel length. Now consider the wave. Chapter 2 showed that speed of large-amplitude solitary waves propagating along a slightly diffused density interface between two deep motionless layers of constant density scales as

$$
c \sim\left(g h \Delta \rho / \rho_{0}\right)^{\frac{1}{2}} a / h .
$$

If coupling requires that the wavespeed and horizontal convective velocities be similar (i.e. $c \sim u$ ), then the amplitude selected by the wave is

$$
a / h \sim\left(g h \Delta \rho / \rho_{0}\right)^{-\frac{1}{2}}(b L)^{\frac{1}{3}}
$$

and this closes the argument.

The experimental measurements showed that the average interfacial buoyancy flux was given by $B \propto(\alpha \Delta T)^{\frac{4}{3}} R_{\rho}^{-8.3}$, and, although the flux through the thin interface ahead of the wave were greater than $B$, it is reasonable to expect that it scaled with the average value. Thus the wave amplitude and speed can be expressed in terms of the independent parameters

$$
\begin{gathered}
a / h \sim\left(g h \Delta \rho / \rho_{0}\right)^{-\frac{1}{2}}(B L)^{\frac{1}{3}} \sim(\alpha \Delta T)^{\frac{1}{18}}\left[\left(R_{\rho}-1\right)^{-\frac{1}{2}} R_{\rho}^{-\frac{8.3}{3}}\right] h\left(\alpha \Delta T, R_{\rho}\right) L^{\frac{1}{3}} \\
c \sim(B L)^{\frac{1}{3}} \sim(\alpha \Delta T)^{\frac{4}{9}} R_{\rho}^{-\frac{8.3}{3}} L^{\frac{1}{3}}
\end{gathered}
$$

where $\Delta \rho / \rho_{0} \equiv \beta \Delta S-\alpha \Delta T \equiv \alpha \Delta T(R \rho-1)$ has been used and the thickness of the thin interface ahead of the wave, $h$, is left as an unspecified function.

Although these results were derived without a detailed knowledge of the wave, and ignore variations in the wave and convective motions during each cycle, they reproduce two fundamental features of the flow. First, with both experiments and Linden \& Shirtcliffe's model suggesting that the thickness of the interface is most 
strongly affected by $R_{\rho},(3.15)$ shows that the wave amplitude was weakly dependent on $\alpha \Delta T$ and strongly dependent on $R_{\rho}$. This result is consistent with the observation that the various flow regimes started at critical values of $R_{\rho}$. Second, non-dimensionalizing the wavespeeds for each experiment by the horizontal convective velocities (3.15) collapses all the measurements onto a single curve, and this confirms that the wavespeed was proportional to $(\alpha \Delta T)^{\frac{4}{9}}$ (figure $3.17 b$ ). However, during each experiment the wavespeed decreased more slowly than the horizontal convective velocities, and thus $c / u$ increased with increasing $R_{\rho}$ rather than remaining constant as predicted. This trend is not a complete surprise as it is reasonable to expect that the exact nature of the coupling would depend on the structure of the interface, and this is a strong function of $R_{\rho}$.

\subsubsection{Effects of the channel aspect ratio}

The same qualitative behaviour occurred in both short $(15 \mathrm{~cm})$ and long $(180 \mathrm{~cm})$ channels, however, as expected, there were quantitative differences between these flows. For example, in the latter case the convection cells were long and flat $(L / H=$ 15.6), and the wave took about 10 minutes to travel the channel length.

Figure 3.19 shows the evolution of $\alpha \Delta T$ and $R_{\rho}$ for the second set of experiments, and, with the conditions at the onset of coupling in bold, indicates that the critical value of $R_{\rho}$ for coupling increased as the channel length increased. This behaviour occurred because a longer channel leads to larger horizontal convective velocities once the convection becomes organized over the channel length (3.15), which in turn demands a greater interfacial wavespeed for coupling. However, these larger wavespeeds cannot occur until the interface is thicker than it is at the onset of coupling in shorter channels, and therefore the critical value of $R_{\rho}$ is greater.

This argument was further supported by the fact that there was a.two-fold increase in the wavespeeds for the 15 and $180 \mathrm{~cm}$ channels, i.e. experiments 2.1 and 2.6. Indeed, this dependence was successfully predicted by the $c \propto L^{\frac{1}{3}}$ result derived from the steady-state resonance model (figure 3.20).

\subsection{Results: interfacial fluxes}

In this section the measured fluxes are presented and compared with previous experimental measurements and theoretical predictions.

\subsubsection{Definitions}

The fluxes across a diffusive interface can be determined by measuring the rate of change of the properties in the adjacent layers or by measuring the gradients through the interface and using the known molecular diffusivities of the two components to calculate the diffusive fluxes through the core. However, even in regimes where the diffusive fluxes through the core are equal to the convective fluxes from the boundary layers, the validity of the second method is questionable because the present experiments indicate that there are often significant horizontal variations in the thickness of the interface. Thus the fluxes were determined from the layer properties.

McDougall $(1981 a, b)$ discussed the conservation equations for two-layer solutesolute and heat-solute systems, and noted that flux definitions require careful con- 


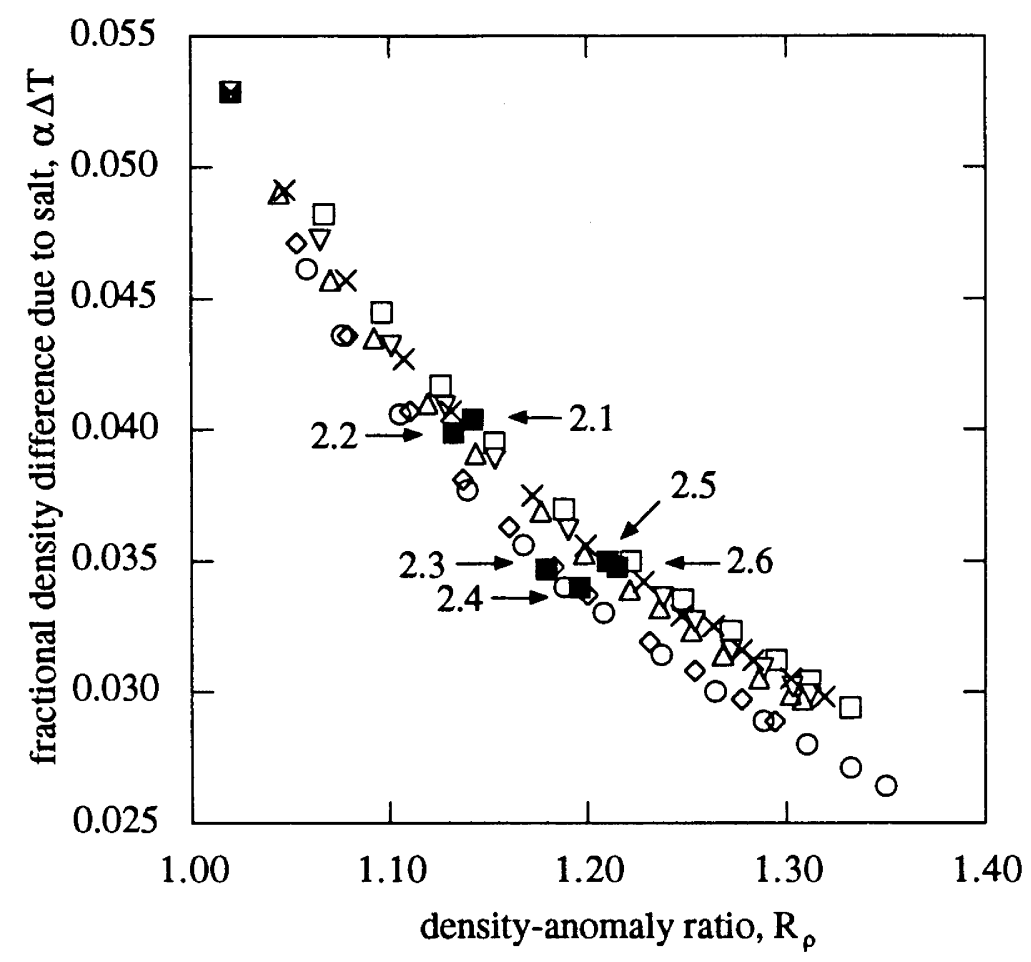

Figure 3.19: $\bar{\alpha} \Delta T$ vs. $R_{\rho}$ for the second set of experiments. The conditions at the onset of coupling are in bold and indicate that the critical value of $R_{\rho}$ for coupling increased as the channel length increased. This behaviour occurred because a longer channel leads to larger horizontal convective velocities, and the greater interfacial wavespeed required for coupling cannot occur until the interface is thicker than it is at the onset of coupling in shorter channels.

sideration. The buoyancy fluxes into the upper and lower layers are

$$
\begin{aligned}
& B_{\mathbf{u}} \equiv-\left(\frac{g}{\rho_{0}}\right) H_{\mathbf{u}} \frac{\mathrm{d} \rho_{\mathbf{u}}}{\mathrm{d} t}=-\left(\frac{g}{\rho_{0}}\right) H_{\mathbf{u}}\left[\alpha_{\mathbf{u}} \rho_{\mathbf{u}} \frac{\mathrm{d} T_{\mathbf{u}}}{\mathrm{d} t}+\beta_{\mathbf{u}} \rho_{\mathbf{u}} \frac{\mathrm{d} S_{\mathbf{u}}}{\mathrm{d} t}\right] \\
& B_{\mathrm{l}} \equiv+\left(\frac{g}{\rho_{0}}\right) H_{\mathrm{l}} \frac{\mathrm{d} \rho_{\mathrm{l}}}{\mathrm{d} t}=+\left(\frac{g}{\rho_{0}}\right) H_{\mathrm{l}}\left[\alpha_{\mathrm{l}} \rho_{\mathrm{l}} \frac{\mathrm{d} T_{\mathrm{l}}}{\mathrm{d} t}+\beta_{\mathrm{l}} \rho_{\mathrm{l}} \frac{\mathrm{d} S_{\mathrm{l}}}{\mathrm{d} t}\right] .
\end{aligned}
$$

In general, the two buoyancy fluxes are not equal, that is, given interfacial salt and sugar mass fluxes produce different buoyancy fluxes into the upper and lower layers. This asymmetry occurs because the expansion coefficients depend on the solute concentrations (e.g. $\left.\alpha_{\mathbf{u}} \neq \alpha_{1}\right)$ and because, for example, conservation of salt $(3.11 b)$ requires that $\mathrm{d}\left(\rho_{\mathbf{u}} H_{\mathbf{u}} T_{\mathbf{u}}\right) / \mathrm{d} t=\mathrm{d}\left(\rho_{\mathbf{l}} H_{1} T_{\mathbf{l}}\right) / \mathrm{d} t$ and therefore $\rho_{\mathbf{u}} H_{\mathbf{u}} \mathrm{d} \rho_{\mathbf{u}} / \mathrm{d} t \neq \rho_{\mathbf{l}} H_{1} \mathrm{~d} \rho_{\mathbf{l}} / \mathrm{d} t$. McDougall showed that the resulting differences in the convective motions in the two layers can lead to preferential entrainment across the interface in one direction and thereby cause the interface to migrate.

Here it was not possible to resolve differences between the buoyancy fluxes into the upper and lower layers, there were no discernible differences in the convective motions in the layers and the interface position remained unchanged to within $\pm 0.5 \mathrm{~mm}$ during each experiment. It was therefore appropriate to use the average buoyancy flux

$$
\bar{B} \approx\left(\frac{g H}{2}\right) \frac{\mathrm{d}}{\mathrm{d} t}\left(\frac{\Delta \rho}{\rho_{0}}\right)=-\left(\frac{g H}{2}\right) \frac{\mathrm{d}}{\mathrm{d} t}(\bar{\alpha} \Delta T-\bar{\beta} \Delta S)=-\left(\frac{g H}{2}\right)\left[\frac{\mathrm{d}}{\mathrm{d} t}(\bar{\alpha} \Delta T)\right]\left(1-\bar{R}_{f}\right)
$$




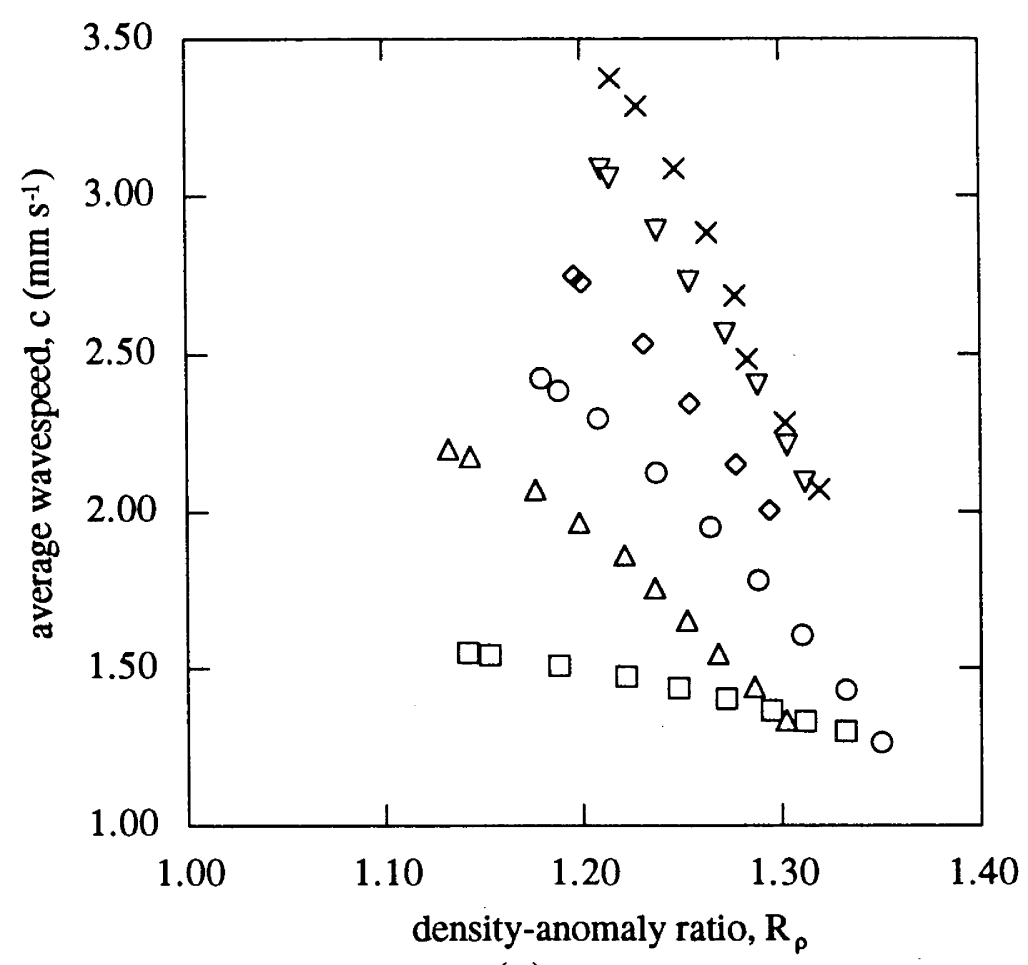

(a)

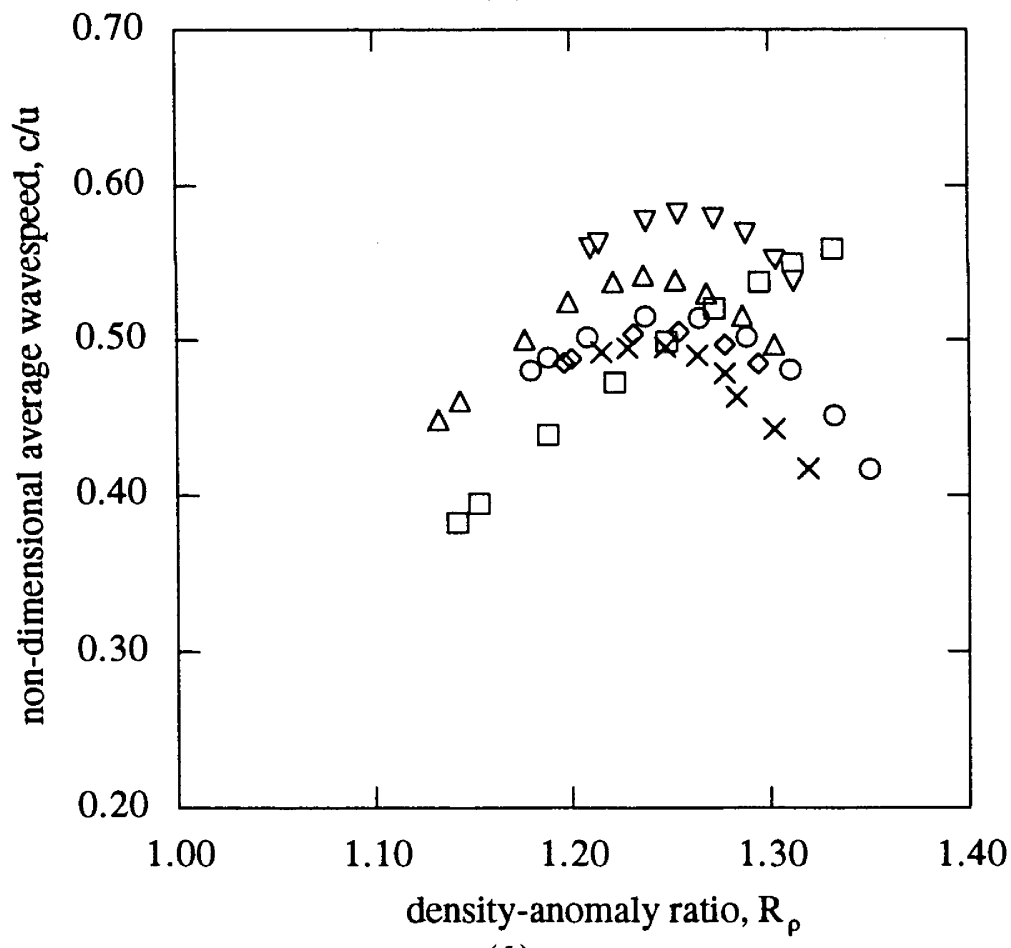

(b)

Figure 3.20: Wavespeed results for the second set of experiments. (a) $c$ vs. $R_{\rho}$. To allow for direct comparison between experiments, the flows were started with the same $\alpha \Delta T$ and $R_{\rho}$, and hence the plot indicates that the wavespeed increased with increasing channel length. This increase occurred because a longer channel leads to larger horizontal convective velocities once the convection becomes organized over the channel length. $(b) c / u$ vs. $R_{\rho}$. A steady-state resonance model suggests that the wavespeeds are proportional to $(B L)^{\frac{1}{3}}$, and the collapse of the non-dimensional wavespeeds for the different experiments confirms this dependence. 
where $R_{\rho} \equiv \bar{\beta} \Delta S-\bar{\alpha} \Delta T$ has been used and

$$
\bar{R}_{f} \approx \frac{\mathrm{d}(\bar{\alpha} \Delta T) / \mathrm{d} t}{\mathrm{~d}(\bar{\beta} \Delta S) / \mathrm{d} t}=\frac{\mathrm{d}(\bar{\beta} \Delta S)}{\mathrm{d}(\bar{\alpha} \Delta T)}
$$

is the ratio of the $T$ and $S$ contributions to the average buoyancy flux. Thus the buoyancy flux was proportional to the rate of change of $\Delta \rho / \rho_{0}$, and the $T$ and $S$ contributions were proportional to the rate of change of $\bar{\alpha} \Delta T$ and $\bar{\beta} \Delta S$ respectively.

\subsubsection{Salt $(T)$ flux}

To calculate the salt flux it was necessary to differentiate the measured $\alpha \Delta T$ values with respect to time. For experiments started at low $R_{\rho}$ the concentration differences across the interface decreased without abrupt changes despite the fact that the flow evolved through several distinct regimes. Thus the function

$$
\alpha \Delta T \propto\left(t-t_{0}\right)^{-\gamma}
$$

was fitted to the data for each experiment and flux values calculated from differentiating the fit. This procedure had two advantages over difference estimates. First, the procedure was insensitive to errors in point values of $\alpha \Delta T$ because the fit was a smooth representation of the data. Second, the derivation of an analytic expression for the flux allowed values to be conveniently calculated at times when the independent parameters were known. However, smoothing the data in this manner resulted in poor flux estimates at the start and end of each experiment, with the first and last estimates having to be eliminated from the analysis. In all experiments the flux estimates calculated from a difference formula and the curve fit agreed within expected uncertainties (figure 3.21).

Figure 3.22(a) shows the salt flux for each experiment plotted against the densityanomaly ratio. As expected, the flux was largest when the driving interfacial density difference $(\alpha \Delta T)$ was large and the interface close to static stability $\left(R_{\rho} \rightarrow 1\right)$. Turner used dimensional arguments to show that this dependence takes the form

$$
\alpha F_{\mathrm{T}}=0.085 \rho\left(g \kappa_{\mathrm{T}}^{2} / \nu\right)^{\frac{1}{3}}(\alpha \Delta T)^{\frac{4}{3}} F_{\mathrm{T}}^{*}\left(R_{\rho}\right),
$$

and this is supported by the fact that the $F_{\mathrm{T}}^{*}$ values for the different experiments more or less collapse onto a single curve (figure $3.22 b$ ). However, a weak trend to larger $F_{\mathrm{T}}^{*}$ at smaller $\alpha \Delta T$ remains. Indeed, deviations from the $4 / 3$ flux law are not unexpected because Kelley (1990) showed that a simple model of turbulent thermal convection predicts fluxes proportional to $(\alpha \Delta T)^{\frac{5}{4}}$, and the assumption that the boundary layers are unaffected by the convective motions is violated when waveconvection coupling occurs. Nevertheless, the most likely reason for the trend is that variations in $\kappa_{\mathrm{T}}$ and $\nu$ values were ignored in evaluating $F_{\mathrm{T}}^{*}$. In particular, the main effect of larger concentrations is to increase in the viscosity of the lower (sugar) layer, and therefore using the viscosity for pure water underestimates the non-dimensional salt flux.

Following Huppert (1971), a power law was fitted to the non-dimensional salt flux data for predictive purposes

$$
F_{\mathrm{T}}^{*}=1.0 R_{\rho}^{-8.3} \text { for } \quad 1.07 \leq R_{\rho} \leq 1.34
$$

This fit is significantly different from the best fit to Shirtcliffe's data, $F_{\mathrm{T}}^{*}=2.59 R_{\rho}^{-12.6}$, and the reason for this difference is unclear because the graphs of $\alpha \Delta T$ vs. $t-t_{0}$ 


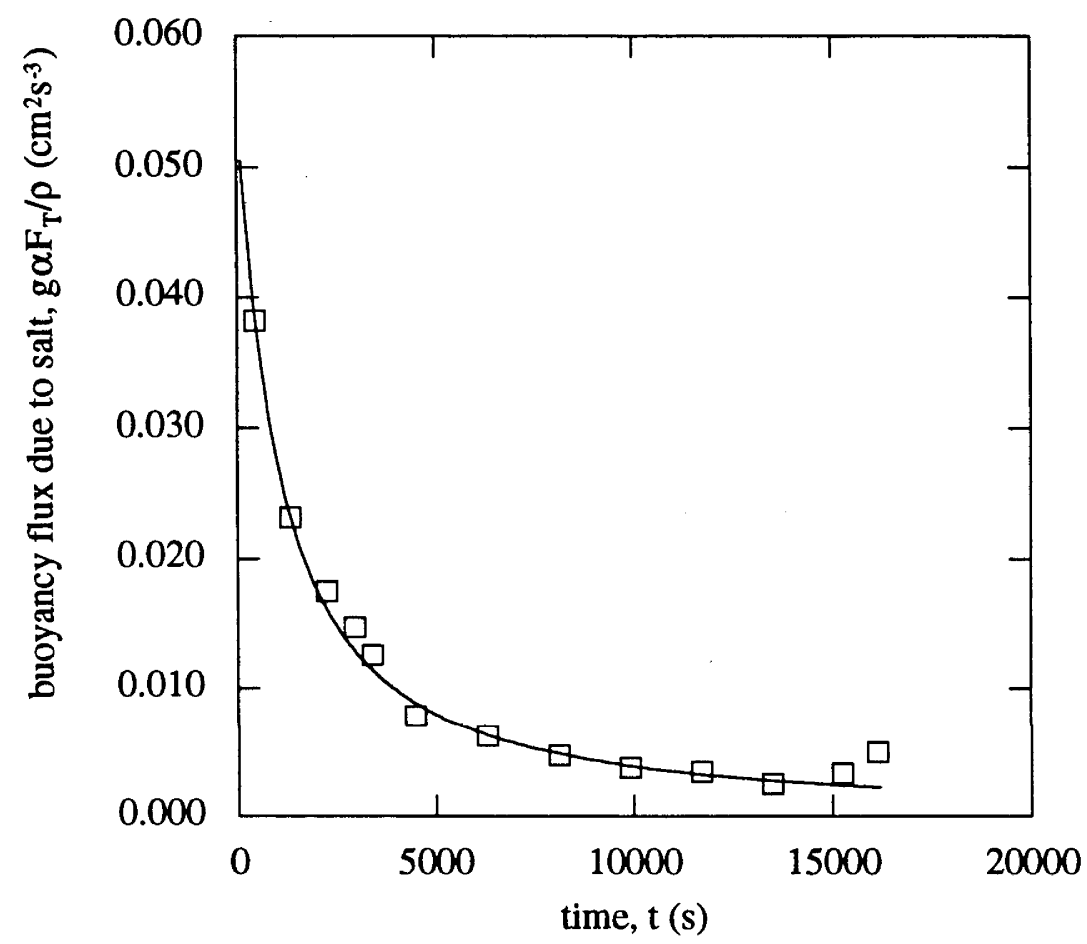

Figure 3.21: Salt ( $T$ ) flux estimates. During each experiment the concentration differences across the interface decreased in response to the salt flux from the upper to the lower layer and sugar flux from the lower to the upper layer, which in turn resulted in the decrease of the fluxes themselves. Estimates of the salt flux, $(g H / 2) \mathrm{d}(\bar{\alpha} \Delta T) / \mathrm{d} t$, were determined from a difference formula $(\square)$ and the derivative of the power law $\bar{\alpha} \Delta T \propto\left(t-t_{0}\right)^{-\gamma}$ that best fits the data (- The curve fit produced a smooth representation of the data and allowed fluxes to be conveniently calculated at times when the independent parameters were known. 
and $\beta \Delta S$ vs. $\alpha \Delta T$ are similar in both studies. However, the present results are correct, and it is possible that Shirtcliffe made an error while non- dimensionalizing the measured fluxes.

\subsubsection{Flux ratio}

Although transport across a diffusive interface is described by the $T$ and $S$ fluxes, results are usually presented in terms of the $T$ flux and the ratio of the $T$ and $S$ fluxes. This description is preferred because $T$ is the component that drives the convection, and the behaviour of the flux ratio indicates whether double-diffusion or turbulent mixing is the dominant transport mechanism.

Equation (3.21) shows that the average flux ratio can be estimated as the gradient of the graph of $\bar{\beta} \Delta S$ against $\bar{\alpha} \Delta T$. However, more accurate estimates were obtained in a manner analogous to that used by Griffiths \& Ruddick (1980) for the finger regime. The rate of change of density in each layer is

$$
\frac{\mathrm{d} \rho}{\mathrm{d} t}=\rho \alpha \frac{\partial T}{\partial t}+\rho \beta \frac{\partial S}{\partial t}=\rho \alpha \frac{\partial T}{\partial t}\left(1-\frac{\beta}{\alpha} \frac{\mathrm{d} S}{\mathrm{~d} T}\right)=\rho \alpha \frac{\partial T}{\partial t}\left(1-R_{f}\right)
$$

and therefore

$$
\left(1-R_{f}\right)=\frac{1}{\rho \alpha} \frac{\mathrm{d} \rho}{\mathrm{d} T}
$$

This result is true for each layer separately, and flux ratios estimated in this manner were more accurate than those estimated from (3.21) for two reasons. First, $\rho$ was known with greater precision than the derived quantities $\bar{\alpha} \Delta T$ and $\bar{\beta} \Delta S$ because it was measured directly. Second, even a $10 \%$ error in the right hand side of (3.26) only results in a $1 \%$ error in the flux ratio because $R_{f} \approx 1$.

Figure 3.23(a) shows the $\rho_{1}$ vs. $T_{1}$ data for one experiment. The slope $\mathrm{d} \rho / \mathrm{d} T$ was constant and, because $\rho \alpha=\partial \rho / \partial T$ varied by less than $2 \%$, the lower layer flux ratio was also constant. Here an average flux ratio was appropriate, and figure $3.23(b)$ shows the average flux ratio for each experiment plotted against the initial fractional density difference due to sugar. Shirtcliffe found a slow increase of $R_{f}$ with increasing $(\beta \Delta S)_{0}$ and suggested that this occurred because changes in viscosity of the fluids affect the diffusivities. However, no such trend was apparent here, and the measurements well represented by the average value

$$
\bar{R}_{f}=0.61 \pm 0.01 \text { for } 1.07 \leq R_{\rho} \leq 1.34
$$

This value is consistent with Shirtcliffe's measurements, $R_{f}=0.60 \pm 0.02$, and Linden \& Shirtcliffe's prediction of $R_{f}=\tau^{\frac{1}{2}}=0.58 \pm 0.02$ for purely double-diffusive transport.

\subsubsection{Effects of wave-convection coupling on the fluxes}

If theoretical models of the diffusive interface are to be used for predicting fluxes in geophysical and technological situations, then it is necessary to determine whether the wave-convection coupling affects the interfacial fluxes. There are two mechanisms by which coupling could increase the fluxes. First, the gradients of $T$ and $S$ across the thin interface ahead of the wave were much larger than would be the case if the interface were rearranged to be of uniform thickness. Second, the large-scale convective circulations maintained these thin (high flux) regions by sweeping the interfacial fluid to the wave. 


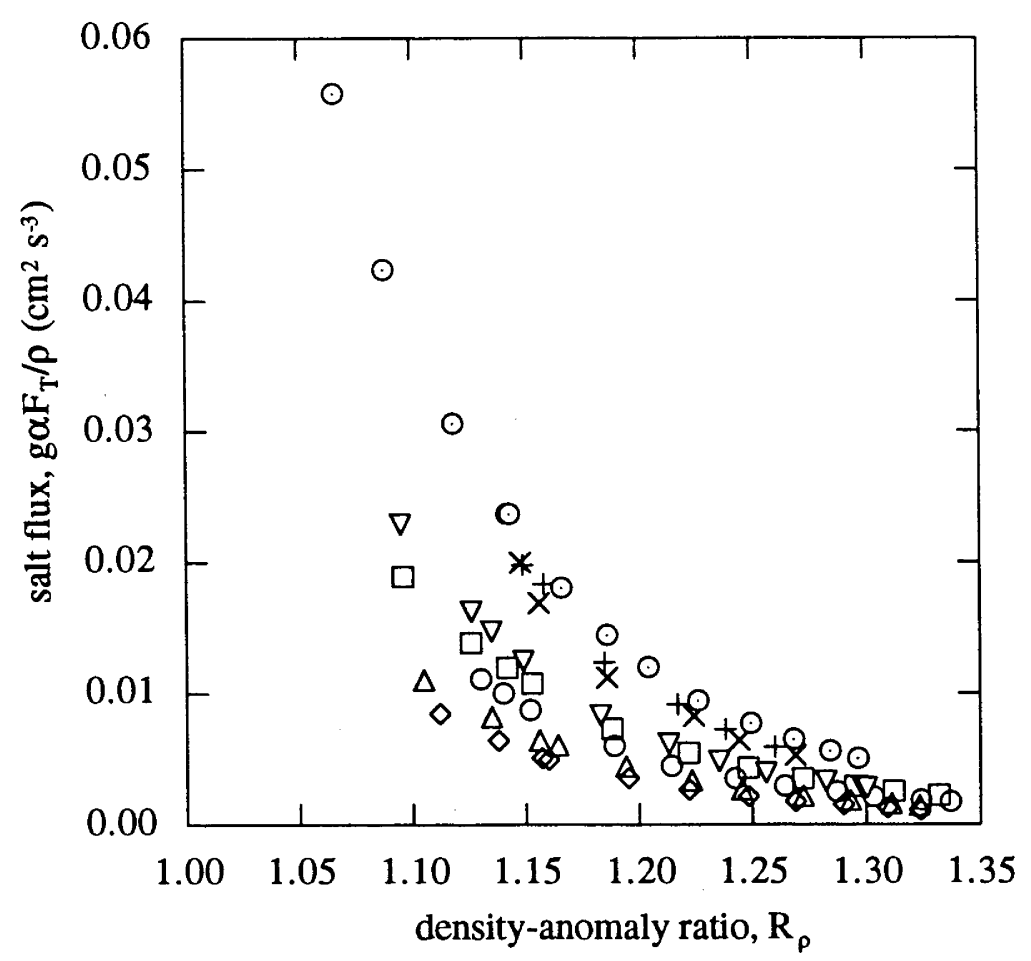

(a)

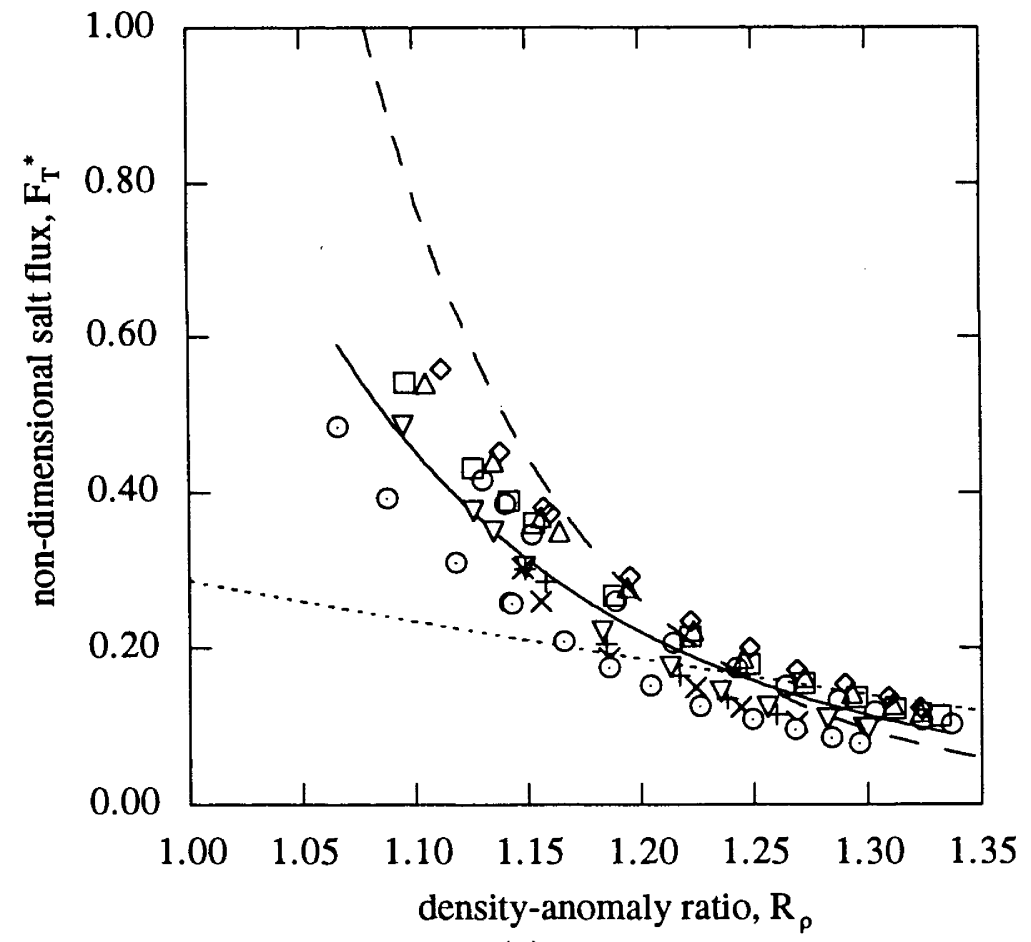

(b)

Figure 3.22: Salt $(T)$ flux results. (a) $g \alpha F_{\mathrm{T}} / \rho$ vs. $R_{\rho}$. As expected, the flux was largest when the driving interfacial density difference $(\alpha \Delta T)$ was large and the interface close to static stability $\left(R_{\rho} \rightarrow 1\right)$. (b) $F_{\mathrm{T}}^{*}$ vs. $R_{\rho}$. Turner used dimensional arguments to show that this dependence takes the form $\alpha F_{\mathrm{T}} \propto(\alpha \Delta T)^{\frac{4}{3}} F_{\mathrm{T}}^{*}$, and this is supported by fact that the resulting non-dimensional fluxes for the different experiments collapse onto a single curve. Linden \& Shirtcliffe's model of a one-dimensional interface $(\cdots .$.$) was in$ reasonable agreement with the experiments for $1.20 \leq R_{\rho} \leq 1.35$, which was when the system supported interfacial waves, but underestimated the fluxes at low $R_{\rho}$. The solid line (- - is the power law $F_{\mathrm{T}} \propto R_{\rho}^{-r}$ that best fits the data, and is quite different to Shirtcliffe's result $(---)$. 


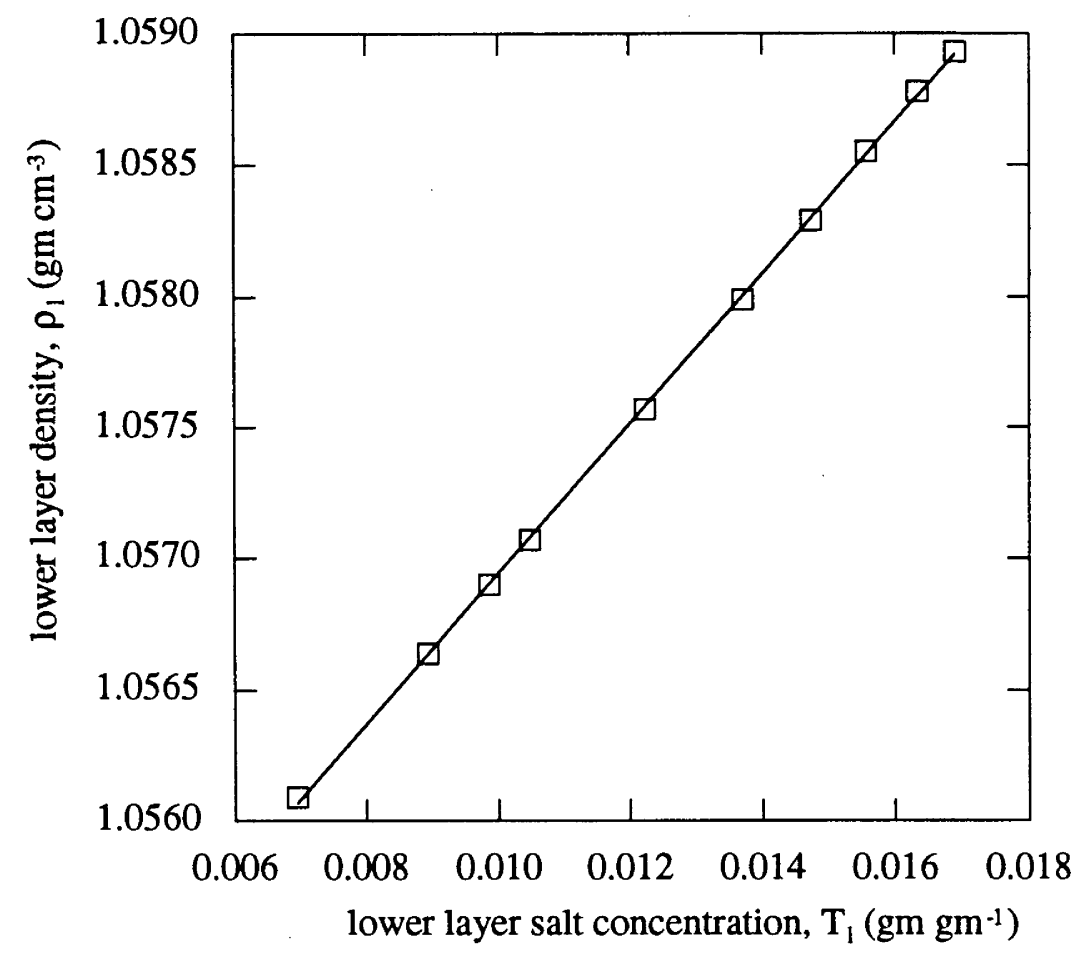

(a)

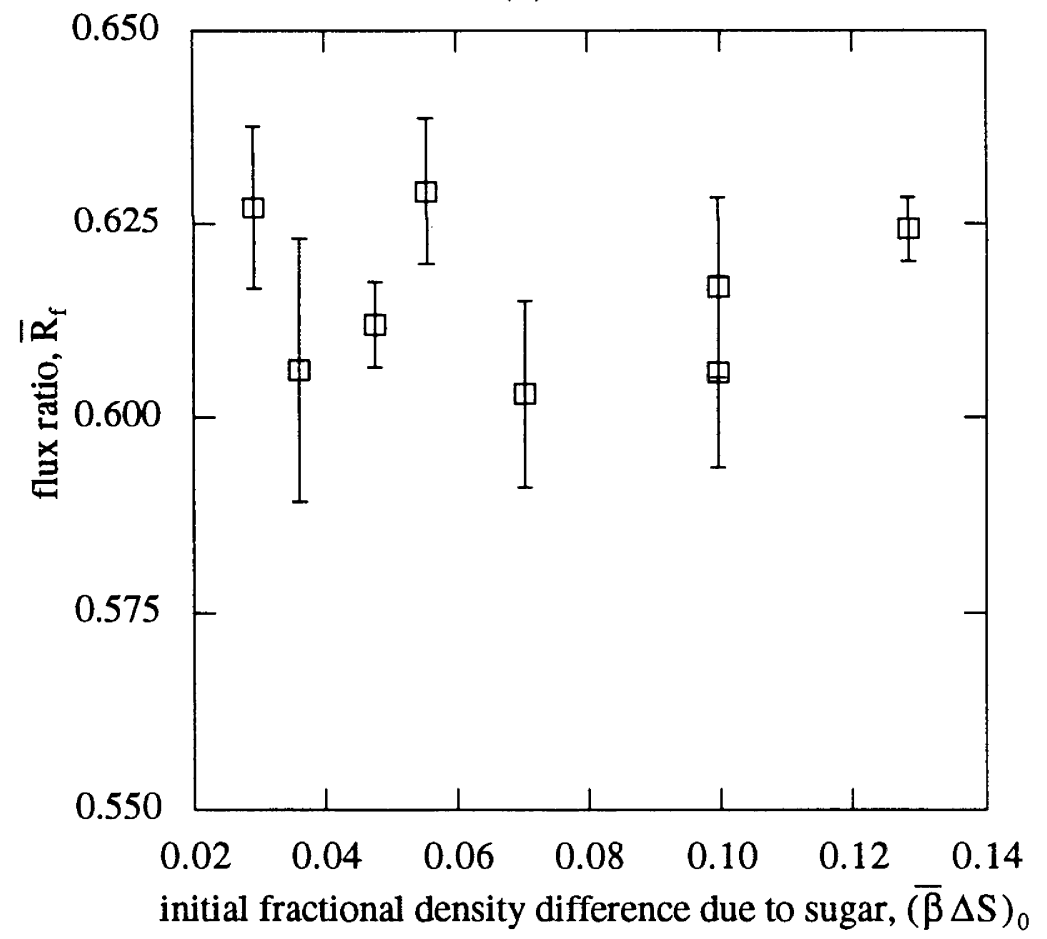

(b)

Figure 3.23: Flux ratio results. (a) $\rho_{\mathrm{l}}$ vs. $T_{1}$. The slope $\mathrm{d} \rho / \mathrm{d} T$ was constant and, because $\rho \alpha=\partial \rho / \partial T$ varied by less than $2 \%$ during the experiment, the lower layer flux ratio was also constant. (b) $\bar{R}_{f} v s$. $(\bar{\beta} \Delta S)_{0}$. The flux ratios for each experiment were similar, $\bar{R}_{f}=0.61 \pm 0.01$, which is consistent with Shirtcliffe's measurements, $R_{f}=0.60 \pm 0.02$, and Linden \& Shirtcliffe's prediction of $R_{f}=\tau^{\frac{1}{2}}=0.58 \pm 0.02$ for purely double-diffusive transport. 
The best method of determining the effects of coupling on the fluxes is to compare fluxes measured for flows with and without coupling. However, no useful method of suppressing coupling was found. For example, placing steel wool at the channel endwalls failed to dissipate waves, and coupling occurred irrespective of the layer depths and tank geometry (Chapter 4). In addition, although flows dominated by viscous dissipation at the tank sidewalls did not exhibit long-term coupling, the $15 \times 2.5 \mathrm{~cm}$ channel available for such experiments did not contain enough fluid for accurate concentration measurements to be made.

Given the absence of suitable control experiments, the measured fluxes were compared with Linden \& Shirtcliffe's model, which assumes that the interface is of uniform thickness and that the boundary layers that grow and break away in a periodic fashion. The model underestimated the fluxes at low $R_{\rho}$ but was in reasonable agreement over the range $1.20 \leq R_{\rho} \leq 1.35$. Linden \& Shirtcliffe observed similar behaviour, and noted that the model is inappropriate in the limit $R_{\rho} \rightarrow 1$ because the assumptions of constant gradients through the diffusive core and a core that is much thicker than the boundary layers are violated. However, the core gradients used in the model should be close to the cycle averaged values, and hence it is unlikely that either of these assumptions had a significant effect on the model's predictions. As waves were not observed until $R_{\rho}=1.13$, the most plausible explanation for the model's failure for $R_{\rho}<1.10$ is that the observed redistribution of the interfacial fluid by the convection was ignored (figures 3.6,3.7).

Surprisingly, the model was in reasonable agreement with the experiments over the range of conditions for which the system supported interfacial waves. Indeed, it appears that the model's failure for $1.13 \leq R_{\rho}<1.20$ was due to coupling, and that the agreement for $R_{\rho} \geq 1.20$ occurred because the thick and thin regions of interface became less distinct and the horizontal convective velocities decreased during rundown. However, this conclusion cannot be stated with certainly as it is again unclear whether the model is in fact a valid representation of a one-dimensional interface.

In conclusion, for a wide range of conditions the interface was organized into thick and thin regions, with the resulting large-scale convective circulations maintaining the thin regions. Although this behaviour might be expected to increase the fluxes, this suggestion was not confirmed because all flows exhibited coupling. In addition, although Linden \& Shirtcliffe's model considered the case of a uniform interface and random convection, they made several assumptions regarding the structure of such an interface, and therefore the model does not necessarily give the true fluxes across a one-dimensional interface. Nonetheless, comparison of the measured fluxes with the model does suggest that the maintenance of thin regions of interface by the convection increased fluxes at low $R_{\rho}$, but that for the conditions under which a single wave propagated back-and-forth the coupling had little effect. This conclusion could be tested using flows dominated by viscous dissipation at the tank sidewalls or a numerical model of a one-dimensional interface as a basis for comparison.

\subsection{Variations on a theme}

The flows described above have all been characterized by a single aqueous saltsugar interface separating two layers of depth $H=11.5 \mathrm{~cm}$. In this section other geometries and solutions are examined. 


\subsubsection{Variations in tank and cell geometries}

For two-layer flows in rectangular channels the important geometrical parameters are the depth ratio $H_{\mathbf{u}} / H_{1}$ and the channel aspect ratios $L / w$ and $L / H_{\mathbf{u}}$. Additional salt-sugar experiments were carried out to determine the geometries for which waveconvection coupling occurs.

The scaling arguments indicate that once the convection becomes organized over the channel length the convective velocities depend on the channel length (3.15), rather than the layer depth (Hunt, 1984). This result implies that coupling can occur when the layer depths are different. To test this assertion an experiment was carried out in the $15 \times 5 \mathrm{~cm}$ channel with $H_{\mathbf{u}}=5.75 \mathrm{~cm}$ and $H_{\mathbf{l}}=11.5 \mathrm{~cm}$ layers, i.e. the Rayleigh number for the upper layer was an order of magnitude larger than that for the lower layer. As expected, the long-term flow was indistinguishable from flows with two layers of equal depth; a single wave propagated back-and-forth along the channel, the flow in the upper layer was a mirror image of that in the lower layer, and the wavespeeds were similar to those for the $H=11.5 \mathrm{~cm}$ flows.

In previous experiments the minimum channel width was $w=5 \mathrm{~cm}$ and the wavespeed decreased in response to changes in the layer properties rather than viscous dissipation at the sidewalls. To investigate the behaviour of flows for which sidewall dissipation is important, experiments were carried out in a channel of crosssection $15 \times 2.5 \mathrm{~cm}$. At first the flow evolved in the usual manner, with several interfacial waves forming and organizing the convection into circulations on the scale of the distance between waves. However, the sidewall dissipation slowed the convective motions, and they became too weak to maintain the thick regions of interface before a single wave formed.

To determine the effects of variations in absolute layer depths, experiments were also carried out in the $15 \times 5 \mathrm{~cm}$ channel with two layers of depth $H=2.5 \mathrm{~cm}$. The small volume of each layer resulted in more rapid changes in layer properties, and, as a result, interfacial waves formed immediately after filling. Further evolution was not observed, with the thick and thin regions of the interface disappearing before a single wave formed. This behaviour probably occurred because the system evolved through the critical $R_{\rho}$ regime for coupling before the flow could become organized. However, it is also possible that the nature of the waves changed in response to the change in the relative magnitudes of the total depth of fluid, the thickness of the thin interface, the thickness of the thick interface and the length of the thick region.

\subsubsection{Variations in the double-diffusive system}

Other experiments demonstrated that wave-convection coupling occurs for a wide range of solutions including glycerol above potassium carbonate water and cold fresh water above hot salt water. In the former case however, coupling only occurred for dilute glycerol solutions where the viscosity contrast across the interface was small. As expected, coupling did not occur for interfaces formed by heating a stable twolayer salt stratified fluid from below. In this configuration the convective motions of the lower layer were dominated by the buoyancy flux through the bottom boundary rather than that through the diffusive interface.

\subsubsection{Multiple diffusive interfaces}

The behaviour of flows with more than two layers was also investigated. A three layer system consisting of a salt layer above two sugar layers was carried out in the 


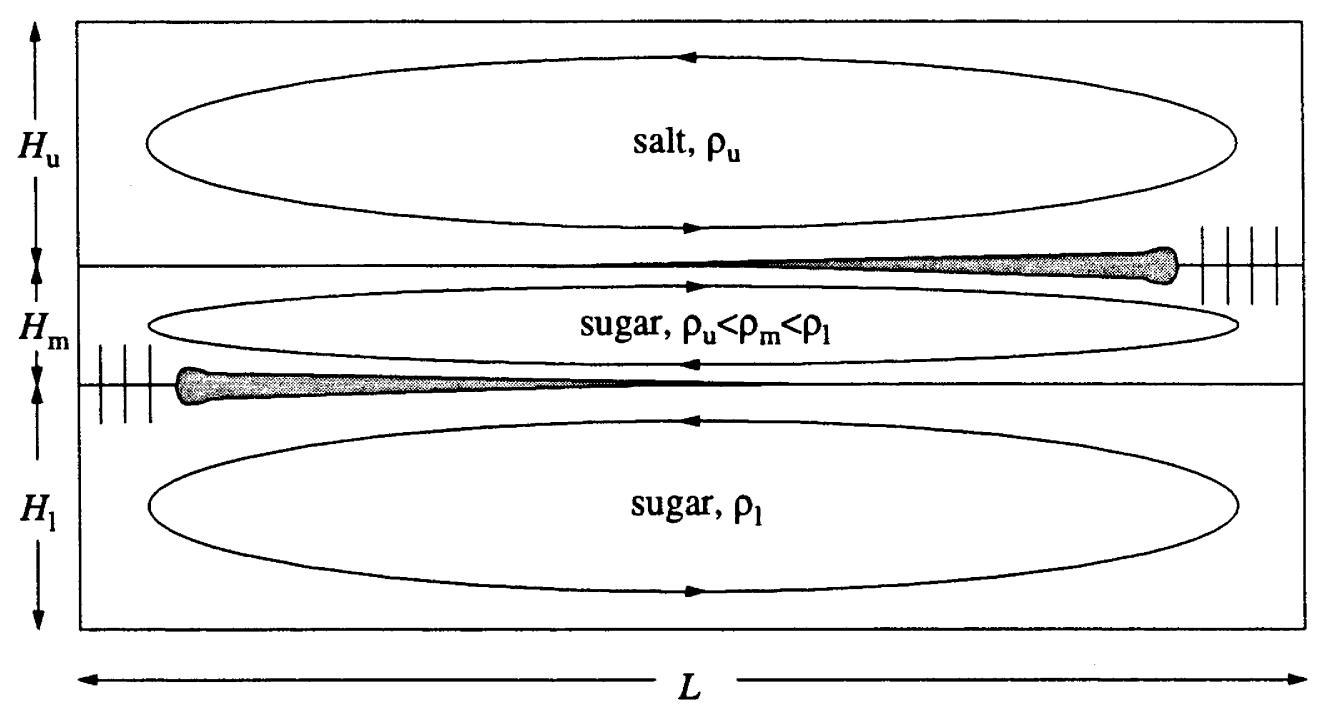

Figure 3.24: Mlustration of the flow with multiple interfaces. A three-layer system was set up with a salt layer above two sugar layers. The diffusive interface between the upper and middle layers transported salt into the middle layer, and, as a result, a diffusive interface formed between the middle and lower layers. After about an hour a single wave propagated back-and-forth along each interface. The waves were exactly out of phase, so that the top wave propagated to the right while the bottom wave propagated to the left. In turn, the convection in each layer was organized into large-scale cells which reversed direction quasi-periodically, with the middle layer rotating in the opposite direction to its neighbours.

$15 \times 15 \mathrm{~cm}$ channel with layers of depths $H_{\mathrm{u}}=H_{\mathrm{l}}=10 \mathrm{~cm}$ and $H_{\mathrm{m}}=5 \mathrm{~cm}$. Initially a diffusive interface formed between the upper (salt) and middle (sugar) layers, while the interface between the middle (sugar) and lower (sugar) layers remained inactive. However, once the salt concentration in the middle layer increased, a diffusive interface formed between the middle and lower layers. After about an hour each interface supported a single wave. The waves propagated back-and-forth along the channel exactly out of phase, so that the top wave propagated to the right while the bottom wave propagated to the left (figure 3.24). In turn, the convection in each layer was organized into large-scale cells which reversed direction quasi-periodically, with the middle layer rotating in the opposite direction to its neighbours. This flow continued until the middle layer became denser than the lower layer and overturning occurred.

\subsection{Summary and conclusions}

A series of experiments has shown that when layers of salt and sugar solution are superimposed with a diffusive interface between, interfacial waves are spontaneously generated by the turbulent convection once the system evolves to a critical value of the density-anomaly ratio $R_{\rho} \equiv \beta \Delta S / \alpha \Delta T$. The waves modulated the interfacial buoyancy flux by modifying the interface thickness and thereby organized the otherwise random convective motions into large-scale circulations. Subsequent interactions between the waves and convection led to a decrease in the number of waves and an increase in the scale of the convective circulations.

In rectangular channels a single wave propagated back-and-forth along the channel for a wide range of conditions. The waves consisted of a short thick solitary-wave nose followed by a long thin tail which was acted upon by gravity and the stress 
exerted by the convection. As the wave traversed the channel, intense plumes at the nose organized the convection in each layer into two large-scale cells which slowed the nose while sweeping the tail forward. The cells ahead of the wave disappeared when the wave collided with an endwall, and the tank-scale circulations then swept the interfacial fluid against the endwall increasing the thickness of the wave before it collapsed outwards.

The waves persisted for about 4 hours and travelled $20 \mathrm{~m}$, whereas solitary waves propagating along a slightly diffused density interface between two deep motionless layers of constant density only persisted for about 2 minutes and travelled $3 \mathrm{~m}$ (Chapter 2). Hence the wave gained enough energy from the convection to overcome the dissipative effects of viscous stresses, turbulent mixing and wave-radiation. Indeed, it is reasonable to expect that the waves would have propagated indefinitely had the convection not run-down. The coupling was found to be most effective when the interface was thick enough to support waves of sufficient amplitude and speed to match the convective velocities, but not so thick as to greatly reduce the interfacial fluxes and convective velocities. A plausible explanation of this result is that it ensures that the wave's tail, which was swept along by the convection, remains attached to propagating nose, and this is required if the horizontal variations in the thickness of the interface are to remain coherent.

Several questions must be answered before the processes by which the waves and convection couple together are fully understood. First, the flows described have all been in rectangular channels, and experiments should be conducted to find out if coupling can occur in other geometries. This question is the subject of Chapter 4. Second, the exact nature of the energy transfer from the convection to the wave is yet to be determined. Indeed, clarification of the coupling mechanisms should also ascertain whether the short time-scale wavespeed variations resulted from random noise or deterministic chaos, and test the suggestion that the changes in coupling during run-down were due to changes in the structure of the interface. However, the best approach to this problem is unclear because mathematical models are difficult to formulate and numerical models require high resolution. Finally, the effects of coupling on the interfacial fluxes should be examined in more detail. The fluxes for flows without coupling could be measured from flows dominated by viscous dissipation at the tank sidewalls or calculated by extending Linden \& Shirtcliffe's model to allow for variable core gradients and thick boundary layers. 


\title{
Chapter 4
}

\section{Coupled waves and convection in annular and three-dimensional cavities}

\author{
'Curiouser and curiouser!' cried Alice. \\ - Alice's Adventures in Wonderland, Lewis Carroll (1865)
}

\subsection{Introduction}

Chapter 3 demonstrated that when layers of salt and sugar solution are separated by a diffusive interface, interfacial waves are spontaneously generated by the turbulent convection once the system evolves to a critical value of the density-anomaly ratio $R_{\rho} \equiv \beta \Delta S / \alpha \Delta T$. The waves modulated the interfacial buoyancy flux by modifying the interface thickness and thereby organized the otherwise random convective motions into large-scale circulations. In rectangular channels the long-term flow consisted of a single wave propagating back-and-forth along the channel, organizing the convection in each layer into two cells which oscillated in length from zero to the full length of the channel. This 'wave-convection coupling' occurred when the wavespeed was comparable to the horizontal convective velocity, that is, when the interface was thick enough to support waves of sufficient amplitude and speed to match the convective velocities, but not so thick as to greatly reduce the fluxes and convective velocities.

Here experiments have been carried out which show that coupling occurs irrespective of the tank geometry. Attention focused on flows in a narrow annular gap because this geometry eliminates endwall reflections and the long-term flow is two-dimensional which simplifies measurements. In this case, a small number of equally-spaced waves propagated in a continuous circular path, with each wave organizing the surrounding convection in each layer into two large-scale cells. Both the waves and convection cells travelled around the annulus. As a rule, all waves propagated in the same direction. However, occasionally one wave propagated in the opposite direction, and during the ensuing head-on collisions the entire leftward and rightward travelling flow patterns passed though each other unchanged. The number of waves decreased as the convection weakened, but this trend was slower than that in rectangular channels and the waves often disappeared before a single wave formed.

The structure of this chapter is as follows. Section 4.2 describes the flows observed in different geometries, and then in $\S 4.3$ measurements of flows in an annulus are presented and compared with the results for flows in rectangular channels. 


\subsection{Experimental observations}

A number of preliminary experiments were carried out to determine the general nature of flows in different geometries. To allow comparison with Chapter 3 , the experiments were set up with a single aqueous salt-sugar interface separating two layers of depth $H=11.5 \mathrm{~cm}$. As in the former experiments, the fluxes decreased with time, weakening the convection and allowing the interface to thicken by diffusion. The convection simultaneously organized the interface into thick and thin regions, while the associated horizontal variations in buoyancy flux produced large-scale convective circulations. Observations of flows in annular and rectangular tanks will now be presented.

\subsubsection{Annular geometry}

Experiments were carried out in a Perspex annulus with inner and outer diameters of 25 and $35 \mathrm{~cm}$. For this configuration the important geometrical parameters are the radial aspect ratio of the cell (the radial width divided by the layer depth) $\Gamma_{r}=0.43$ and the circumferential aspect ratio (the mean circumference divided by the layer depth) $\Gamma_{\theta}=8.2$. The experiments were started by placing the upper (salt) layer in the annular gap and then feeding in the denser lower (sugar) layer through inlets at the base of the tank. To visualize the flow, a 50 Watt fluorescent lamp was positioned in the centre of the annulus and tracing paper attached to the inner and outer sidewalls in the usual fashion.

The flow evolved through the three transient regimes observed for flows in rectangular channels (figures 3.6-3.8), and after about an hour a stable configuration of three or four large thick regions of interface formed. These waves consisted of a long thin tail attached to a short thick nose, and propagated around the annulus in the same direction. Intense plumes at the nose of each wave organized the surrounding convection in each layer into two large-scale counter-rotating cells (figure 4.1).

In this manner, the waves and convection cells in each layer propagated in a continuous circular path without reflection. At first the waves were equally-spaced, but with time the larger amplitude waves caught up with those of lesser amplitude. The behaviour during the ensuing overtaking collisions was similar to that in rectangular channels, with the waves merging and the number of convection cells decreasing (figure 3.10). As a rule, the number of waves decreased to two after about 30 minutes, and the thick and thin regions of the interface then disappeared before a single wave could form from the next overtaking collision. In the rare circumstances when a single wave did form, its tail and the two convection cells in each layer extended half way around the annulus.

Sometimes the pattern of co-rotating waves was disrupted by generation of a new wave. This process began with a thickening of a small patch of interface ahead of an existing wave. The lump was swept towards the wave by the large-scale convective circulations and grew in size as it engulfed the small interfacial lumps in its path. In this manner, the lump soon became large enough to couple with the convection and propagate as a wave. The existing convection pattern was then destroyed as the wave organized the surrounding convection in each layer into two large-scale cells. This wave and its associated cells propagated around the annulus in the opposite direction to the older waves. In general, the entire leftward and rightward travelling flow patterns passed through each other unchanged during the ensuing head-on collisions (figure 3.9). However, when the colliding waves were of 


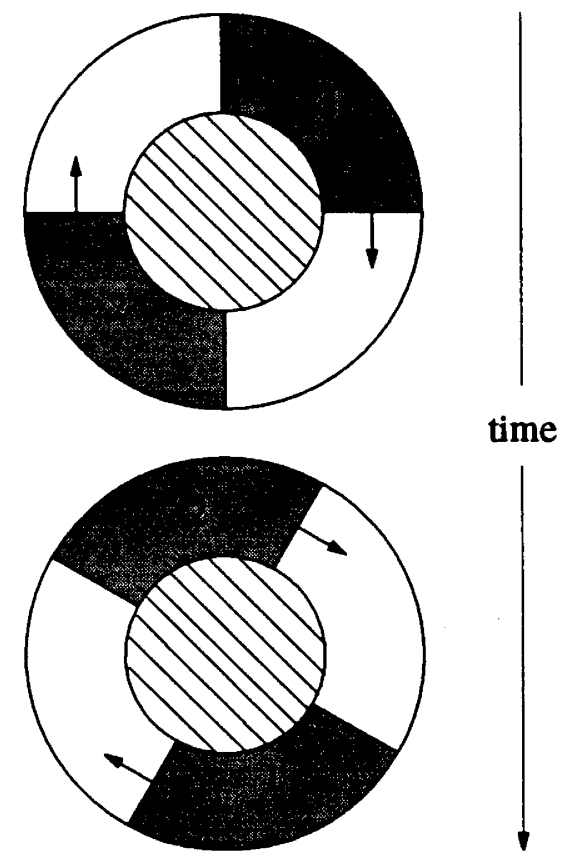

(a)

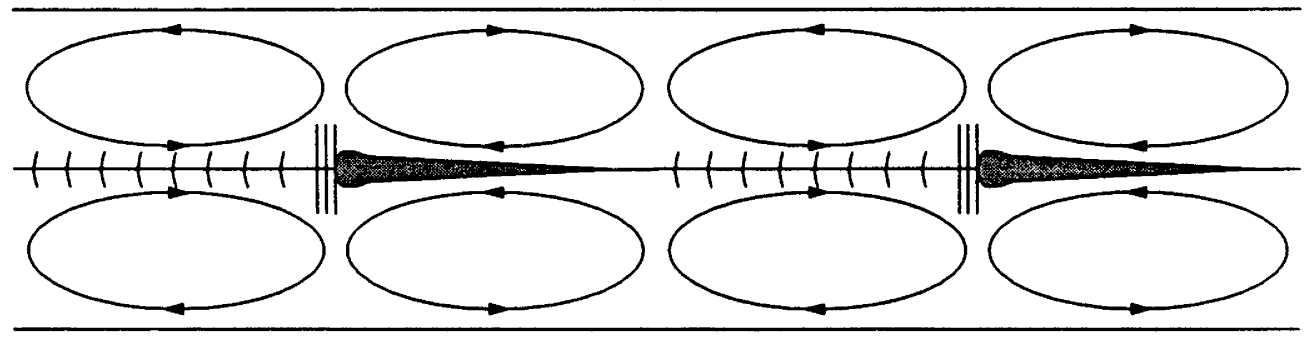

(b)

Figure 4.1: Illustration of the flow in an annulus. (a) Top and (b) side views of the 2-wave regime. The waves consisted of a long thin tail attached to a short thick nose. Intense plumes at the nose of each wave organized the surrounding convection in each layer into two large-scale counter-rotating cells. Thus the two waves and four convection cells in each layer travelled in a continuous circular path without reflection. 


\begin{tabular}{lccccccccc} 
& & \multicolumn{3}{c}{ initial conditions } & \multicolumn{3}{c}{ onset of coupling } & \multicolumn{3}{c}{ final measurements } \\
run & symbol & $\bar{\alpha} \Delta T$ & $R_{\rho}$ & $t$ (h:m:s) & $\bar{\alpha} \Delta T$ & $R_{\rho}$ & $t(\mathrm{~h}: \mathrm{m}: \mathrm{s})$ & $\bar{\alpha} \Delta T$ & $R_{\rho}$ \\
1 & $\square$ & 0.0249 & 1.02 & $1: 10: 24$ & 0.0177 & 1.17 & $2: 22: 21$ & 0.0155 & 1.24 \\
$2 a$ & $\times$ & 0.0462 & 1.03 & $1: 08: 24$ & 0.0320 & 1.18 & $2: 47: 01$ & 0.0273 & 1.27 \\
$2 b$ & + & 0.0462 & 1.03 & $1: 00: 39$ & 0.0327 & 1.17 & $2: 48: 38$ & 0.0268 & 1.28 \\
3 & $\bigcirc$ & 0.0975 & 1.02 & $1: 04: 40$ & 0.0697 & 1.17 & $2: 51: 00$ & 0.0605 & 1.26
\end{tabular}

Table 4.1: Experimental parameters and results for annulus experiments. These experiments were designed to test the dependence of the flow on $\alpha \Delta T$ and $R_{\rho}$, and to this end were started with a wide range of solute concentrations.

significantly different amplitudes, the larger wave engulfed the smaller wave and the convection pattern changed accordingly.

\subsubsection{Rectangular geometry}

Experiments were also carried out in a square glass tank of horizontal cross-section $25 \times 25 \mathrm{~cm}$. After about an hour a single thick region occupied half the tank and rotated around an (imaginary) axis in the centre of the tank. Intense plumes at the nose drove two large-scale counter-rotating convection cells in each layer (figure 4.2). Hence this three-dimensional flow was similar to the two-dimensional flows in an annulus.

In a rectangular channel of horizontal cross-section $30 \times 10 \mathrm{~cm}$ the flow was divided into three such square regions (i.e. $10 \times 10 \mathrm{~cm}$ ), with a single wave rotating around the centre of each square (figure 4.3). The middle wave propagated in the opposite direction to its neighbours, and the waves were phase-locked such that they banked up against each other while propagating along the imaginary cell boundaries. It is unclear why this periodic mode was not observed in other rectangular channels (Chapter 3). However, the most likely explanation is that flows with four or more waves are unstable to perturbations of the phase-locked state.

\subsection{Experimental results}

A number of more detailed experiments were carried out in the annulus to determine the conditions for onset of wave-convection coupling and make quantitative measurements of the wavespeed. The conditions for these experiments are listed in table 4.1 .

\subsubsection{Onset of wave-convection coupling}

Figure 4.4 shows the evolution of $\alpha \Delta T$ and $R_{\rho}$, with the conditions at the onset of coupling in bold. The measurements indicate that coupling occurred when the density-anomaly ratio exceeded the critical value

$$
R_{\rho}^{\mathrm{c}}=1.17 \pm 0.01 \quad \text { for } \quad 0.0249 \leq(\bar{\alpha} \Delta T)_{0} \leq 0.0975
$$

This value is close to the critical value for flows in a rectangular channel of horizontal cross-section $15 \times 5 \mathrm{~cm}$ (Chapter 3 ) because in both cases the convection cells were 
top view
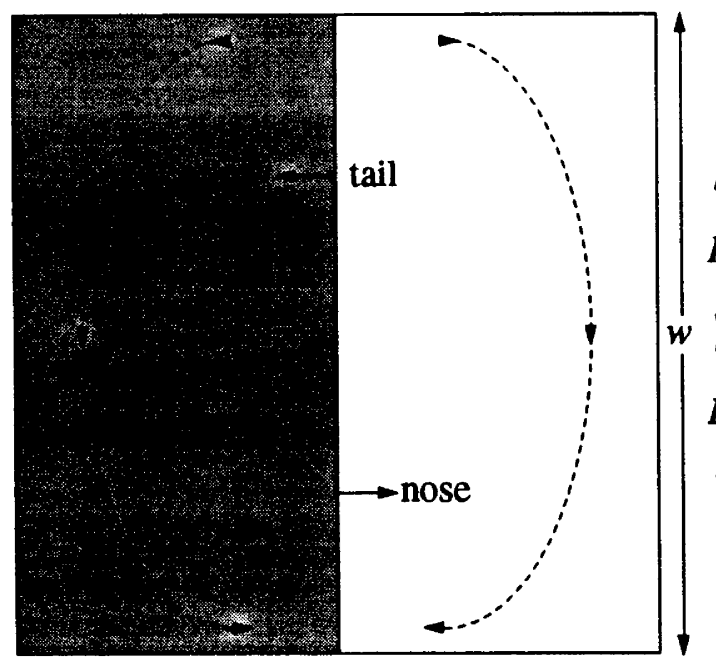

front view
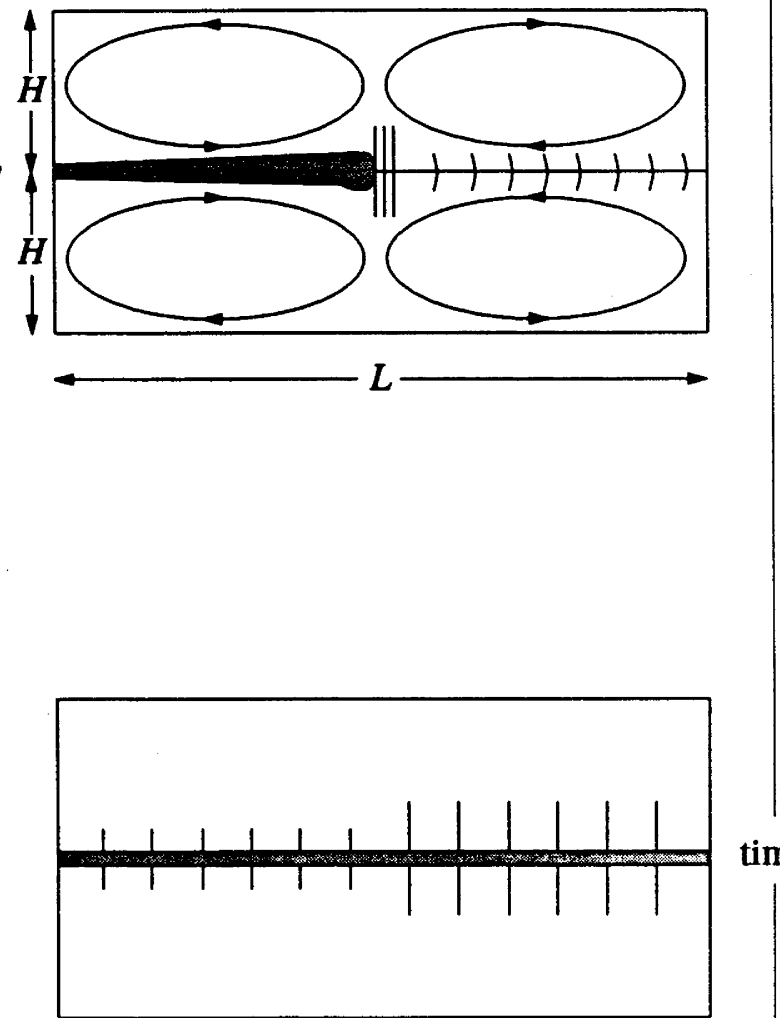
time

Figure 4.2: Illustration of the flow in a square tank. A single wave occupied about half the tank and rotated around the centre of the tank, while intense plumes at the nose drove two large-scale counter-rotating convection cells in each layer. The arrows $(\rightarrow)$ and dashed lines $(\cdots \cdots)$ show the direction of the wave and convective motions (at the level of the interface) respectively. 

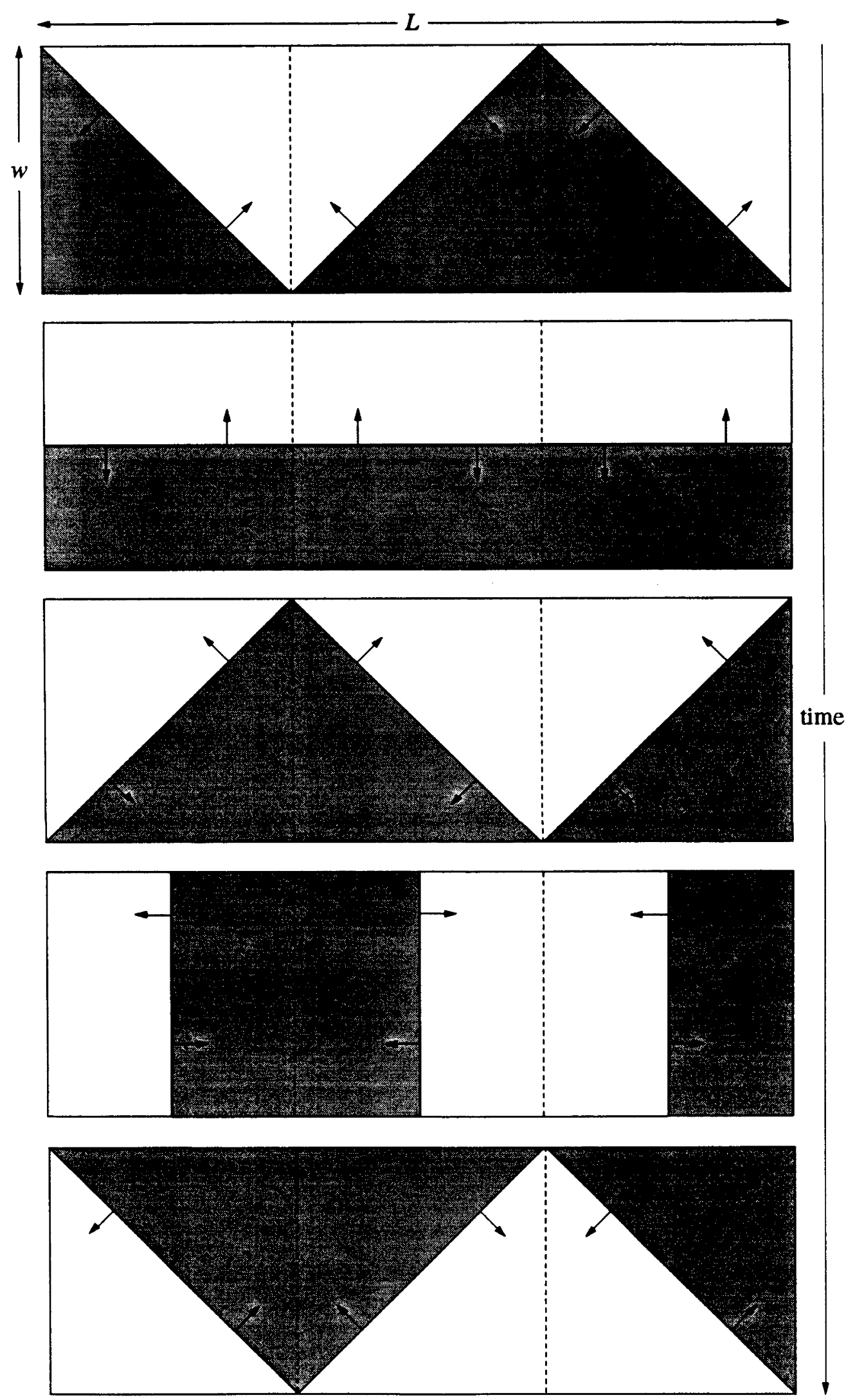

Figure 4.3: Schematic top view of the flow in a $30 \times 10 \mathrm{~cm}$ rectangular tank. The flow was divided into three square regions with a single wave rotating about the centre of each square. The middle wave propagated in the opposite direction to its neighbours, and the waves banked up against each other while propagating along the two imaginary cell boundaries. 


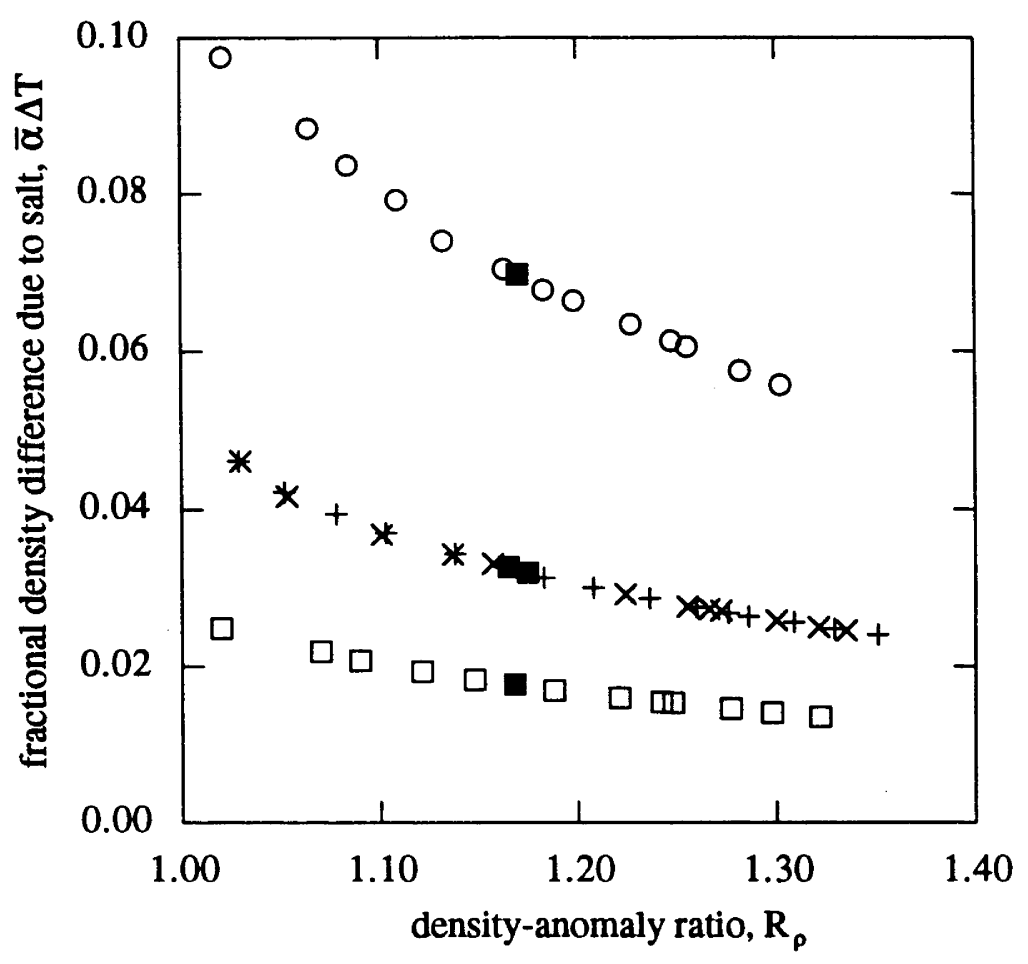

Figure 4.4: $\bar{\alpha} \Delta T$ vs. $R_{\rho}$ for the annulus experiments. The measurements indicate that the critical value of $R_{\rho}$ for coupling was close to that for flows in a $15 \times 5 \mathrm{~cm}$ rectangular channel. This occurred because the three waves which formed organized the convection in each layer into six convection cells of length $15 \mathrm{~cm}$.

about $15 \mathrm{~cm}$ long, i.e. here three equally-spaced waves organized the convection in each layer into six cells of length $2 \pi \bar{r} / 2 \times 3 \approx 15 \mathrm{~cm}$ (where $\bar{r}$ is the mean radius).

\subsubsection{Wavespeed}

Wave position was measured as a function of time for all the experiments listed in table 4.1. However, it was not possible to obtain a complete record for every wave because of the complicated wave-wave interactions and the practical difficulties associated with monitoring several waves at once. Table 4.2 details the waves for which measurements were made.

The evolution of experiment $2 a$ is shown in figure 4.5(a). A stable configuration of three waves formed about an hour after the start of the experiment and 20 minutes later the number of waves decreased to two. These waves were on opposite sides of the annulus and propagated in the same direction. However, wave $2 a .2$ was larger and faster than wave $2 a .1$, and after a further 40 minutes the waves merged forming wave $2 a .3$. This wave and its associated convection cells extended half way around the annulus. The increase in the horizontal scale of the convection resulted in larger horizontal convective velocities and a corresponding increase in the wavespeed. Meanwhile, a new wave formed and propagated in the opposite direction to wave $2 a .3$, decreasing in amplitude during the ensuing head-on collisions. Figure $4.5(b)$ shows the average and instantaneous speed of propagation of wave $2 a .3$. Like the waves in rectangular channels, this wave experienced significant short-time variations in wavespeed.

Figure 4.6(a) shows the average wavespeed for each experiment plotted against the density-anomaly ratio. Two interesting features emerge. First, as expected, the wavespeed was largest when the driving interfacial density difference $(\alpha \Delta T)$ 


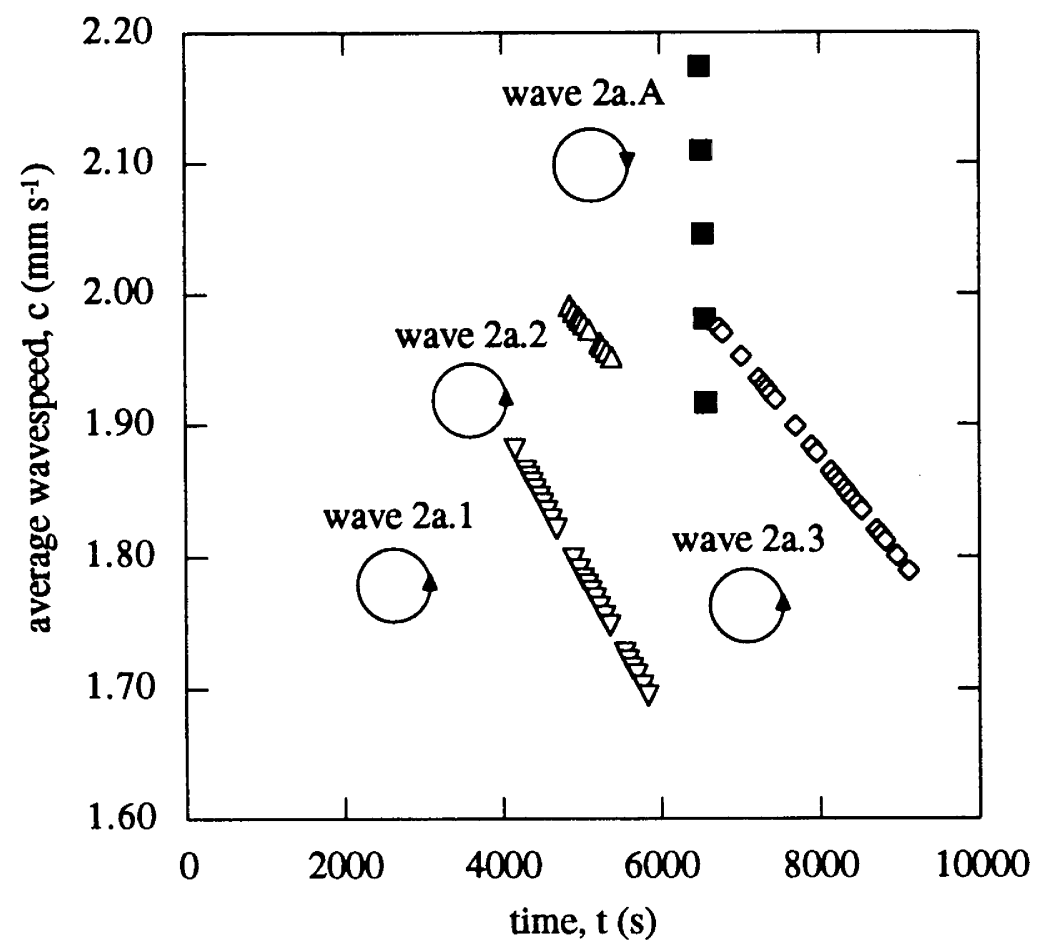

(a)

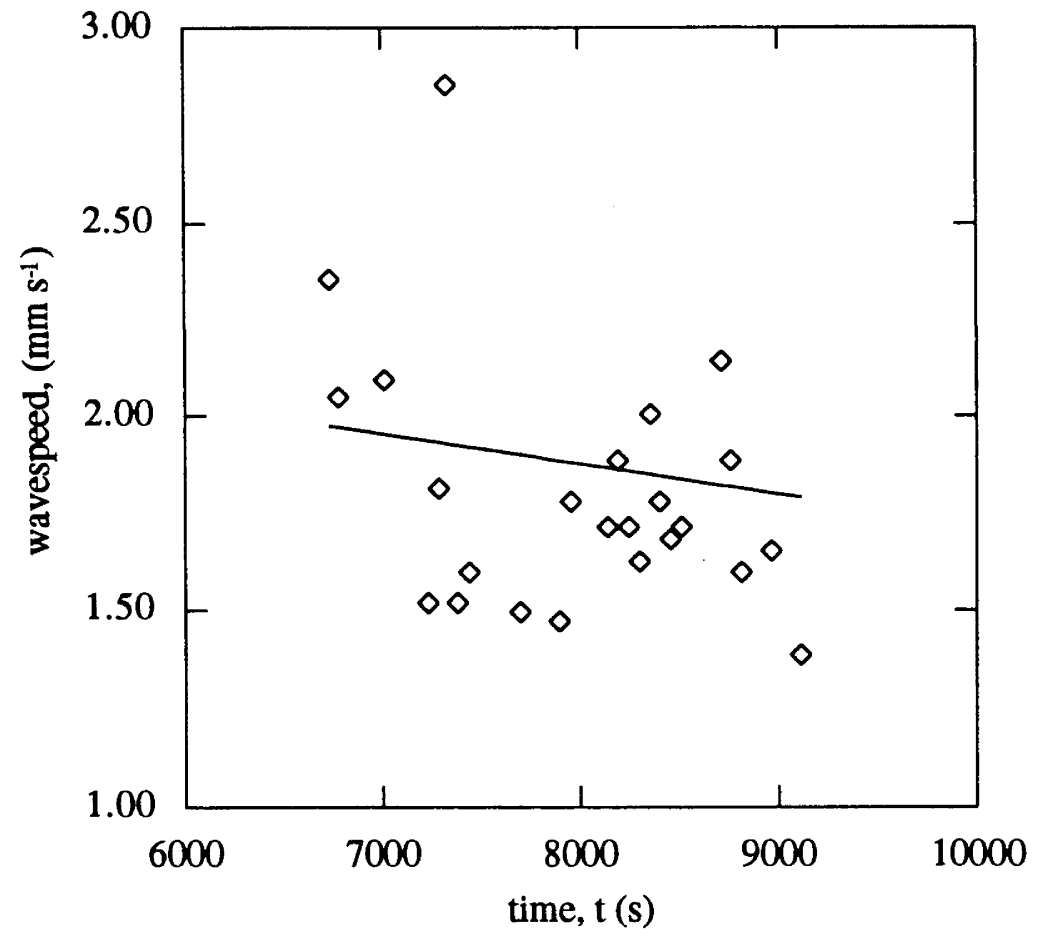

(b)

Figure 4.5: Evolution of experiment 2a. (a) After about 80 minutes the flow consisted of two waves (i.e. $2 a .1$ and $2 a .2$ ) propagating around the annulus in a counter-clockwise direction. Small differences in the amplitude and speed of these waves gave rise to an overtaking collision and wave $2 a .3$ formed. This wave extended half way around the annulus and was faster than earlier waves because the increase in the horizontal scale of the convection resulted in larger horizontal convective velocities. Meanwhile, a new wave ( ) formed and propagated in a clockwise direction before being destroyed. (b) The speed of wave $2 a .3$ as determined from a difference formula $(\diamond)$ and the derivative of the second degree polynomial that best fits the displacement-time measurements, $\mathrm{d} X(t) / \mathrm{d} t$ where $X(t)=A_{0}+A_{1} t+A_{2} t^{2},(-$ Like the waves in rectangular channels, the waves in the annulus experienced short-time scale variations in wavespeed. 


\begin{tabular}{lcccccccc} 
& \multicolumn{1}{c}{ formation of wave } & \multicolumn{3}{c}{ disappearance of wave } \\
wave & $n$ & symbol & $\mathrm{t}(\mathrm{h}: \mathrm{m}: \mathrm{s}:)$ & $\bar{\alpha} \Delta T$ & $R_{\rho}$ & $t(\mathrm{~h}: \mathrm{m}: \mathrm{s})$ & $\bar{\alpha} \Delta T$ & $R_{\rho}$ \\
$1.1 \dagger$ & 3 & $\times$ & $1: 10: 24$ & 0.0179 & 1.16 & $1: 23: 18$ & 0.0173 & 1.18 \\
1.2 & 1 & + & $1: 54: 50$ & 0.0162 & 1.22 & $2: 08: 44$ & 0.0158 & 1.23 \\
$2 \mathrm{a} .1$ & 2 & $\Delta$ & $1: 18: 53$ & 0.0314 & 1.18 & $1: 31: 50$ & 0.0305 & 1.20 \\
$2 \mathrm{a} .2$ & 2 & $\nabla$ & $1: 08: 42$ & 0.0322 & 1.17 & $1: 37: 34$ & 0.0302 & 1.21 \\
$2 \mathrm{a} .3$ & 1 & $\diamond$ & $1: 51: 53$ & 0.0294 & 1.22 & $2: 34: 56$ & 0.0276 & 1.26 \\
$2 \mathrm{~b} .1$ & 1 & $\square$ & $1: 52: 56$ & 0.0291 & 1.23 & $2: 48: 38$ & 0.0267 & 1.28 \\
3.1 & 1 & $\bigcirc$ & $1: 49: 20$ & 0.0645 & 1.21 & $2: 51: 00$ & 0.0596 & 1.26
\end{tabular}

Table 4.2: Details of the measured waves. Each wave has a label consisting of the experiment number followed a number denoting whether it was the first, second or third wave monitored during the experiment. The total number of waves in the tank during the lifetime of each wave $(n)$ was recorded, and the values of the independent parameters at the times when waves formed and disappeared calculated.

was large and the interface close to static stability $\left(R_{\rho} \rightarrow 1\right)$, that is, when the fluxes were largest. For example, there was a two-fold difference in the fluxes during experiments $2 b$ and 3 (i.e. $(\alpha \Delta T)_{0}=0.0462$ and 0.0975 ), and the wavespeed in the latter flow was about $25 \%$ faster than that in $2 b$. Second, as noted in the description of experiment $2 a$, when flows had similar layer properties but a different number of waves, then the wavespeeds were larger for the flow with the smaller number of waves. This difference occurred because the convection was organized over different horizontal lengths, and thus the horizontal convective velocities were different

In Chapter 3 it was shown that once the convection becomes organized into large-scale cells the convective velocities are given by

$$
u \sim(B L)^{\frac{1}{3}},
$$

where $B$ is the interfacial buoyancy flux and $L$ is the horizontal length of the cells. Here each wave organized the surrounding convection into two large-scale convection cells, and thus the length scale was taken as the mean circumference of the annulus divided by twice the number of waves

$$
L=2 \pi \bar{r} / 2 n
$$

Figure 4.6(b) shows the non-dimensional wavespeed for each experiment, $c / u$, plotted against the density-anomaly ratio, and, again, the measurements for the different experiments collapse onto a single curve indicating that the wavespeed and horizontal convective velocities were similar. The collapse is not perfect however, with waves 1.1 and 1.2 decaying more rapidly than the other waves. Nevertheless, this anomalous behaviour was probably an artefact of the poorly constrained wave position measurements which resulted from the convection being weaker than usual.

Finally, the wavespeeds in the annulus were much larger than the wavespeeds for the flows with the same layer properties in a $15 \times 5 \mathrm{~cm}$ rectangular channel, i.e. compare $2 a, b$ with the Chapter 3 experiment $1.3 a$. The fact that the nondimensional wavespeeds for these experiment collapse onto the same curve indicates this difference was due to the convection being organized over a larger horizontal length in the annulus. 


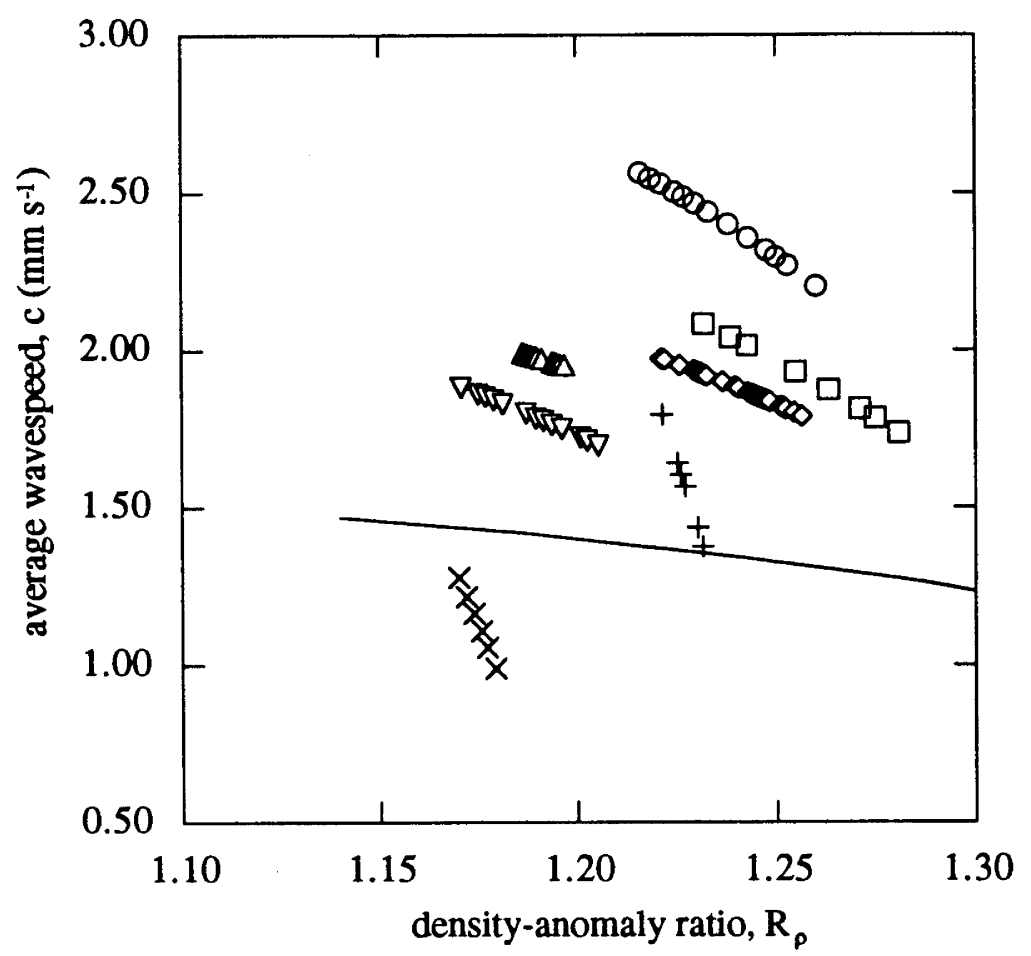

(a)

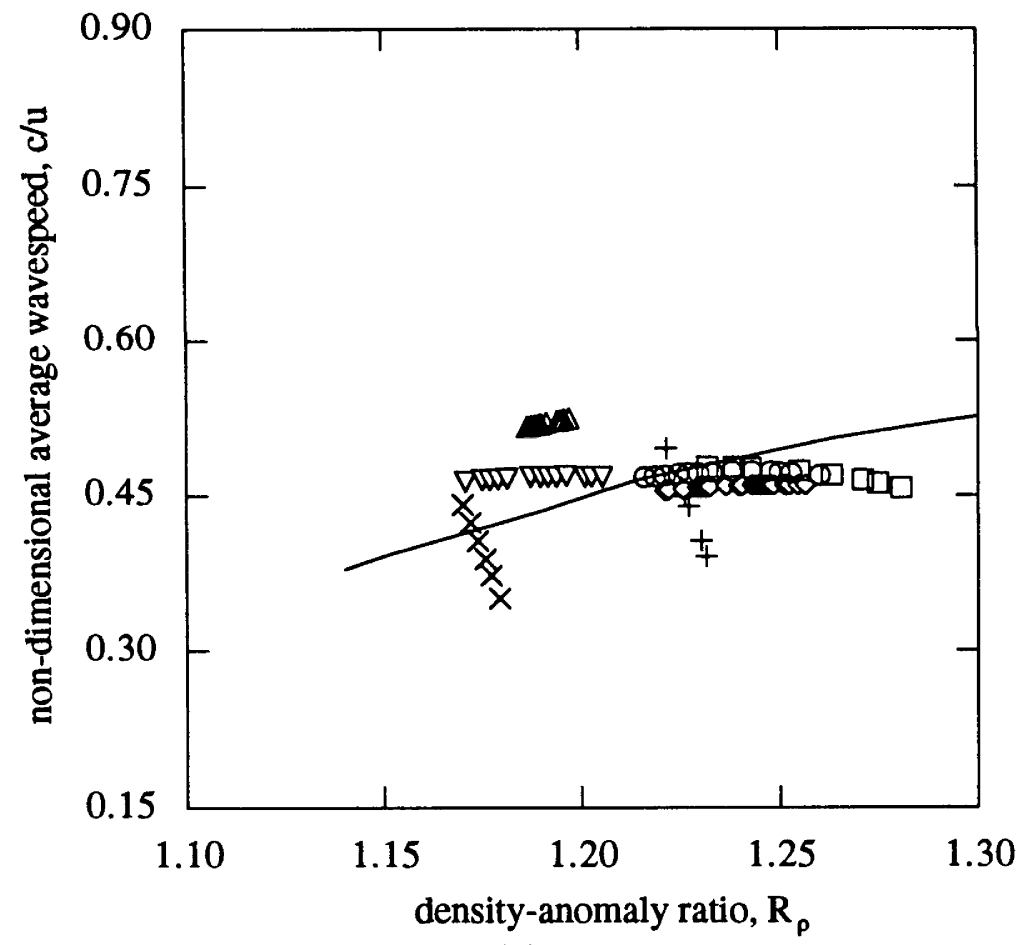

(b)

Figure 4.6: Wavespeed results. For comparison, the speed of the wave in the Chapter 3 experiment $1.3 a$ is plotted (— rectangular channel and started with the same solutions as experiments 2.a,b. (a) c vs. $R_{\rho}$. The wavespeed was largest when the driving interfacial density difference $(\alpha \Delta T)$ was large and the interface close to static stability $\left(R_{\rho} \rightarrow 1\right)$, that is, when the fluxes were largest. In addition, for given layer properties, the wavespeed increased as the number of waves decreased because the convection was organized over a larger horizontal scale. (b) $c / u$ vs. $R_{\rho}$. The model presented in Chapter 3 indicated that the wavespeeds are proportional to $(B L)^{\frac{1}{3}}$. Non-dimensionalizing the wavespeeds for each experiment by this velocity collapses all the measurements onto a single curve, and this confirms that coupling requires a matching of the wave and convection speeds. 


\subsection{Summary and conclusions}

This experimental investigation has demonstrated that the coupling of large-amplitude waves and turbulent convection driven from a diffusive interface is readily established in the laboratory. In particular, reflection from tank endwalls is not required, with the long-term flows in annular and square tanks consisting of a single wave propagating in a continuous circular path organizing the convection into large-scale travelling-wave cells. Thus coupling is a local, rather than global, phenomenon, and as the lifetime of these waves was about half that of the waves in rectangular channels, energy transfer from the convection to waves was less efficient than that in rectangular channels. Indeed, this suggestion is consistent with the observation that when a wave collides with an endwall the tank-scale convection cells sweep the interfacial fluid against the endwall, increasing the thickness and potential energy of the wave. 


\title{
Chapter 5
}

\section{Summary and conclusions}

\author{
...the multitudinous laughter of ocean waves \\ - Aeschylus, Prometheus Bound 88
}

In this chapter the results pertaining to deep-water internal solitary waves and the coupling of such waves with convection driven from a diffusive interface are summarized and the direction of future work discussed. The latter flow is then compared to the El Niño-Southern Oscillation phenomenon and atmospheric squall lines.

Chapter 2 presented detailed experimental measurements of solitary waves propagating along a slightly diffused density interface between two deep motionless layers of constant density. Two types of waves were observed; small-amplitude waves which carried energy and momentum, and large-amplitude waves which also carried mass. The small-amplitude waves were well described by weakly nonlinear theory, but fully nonlinear theory was required for the large-amplitude waves. In particular, the wavelength of small-amplitude waves decreased with increasing amplitude, whereas for large-amplitude waves the wavelength increased with increasing amplitude. However, despite these differences, the wavespeed increased with increasing amplitude at the same rate for both small and large amplitudes. Observations were also made of various wave-boundary and wave-wave interactions, and there was evidence that waves suffered a negative spatial phase shift during collisions. The head-on collision between waves of equal amplitudes was similar to a reflection, whereas for waves of different amplitudes there was an exchange of fluid such that the leftward and rightward propagating waves had the same amplitudes before and after the collision.

In Chapters 3 and 4 the coupling of interfacial waves with convection on both sides of a double-diffusive interface was considered. Experiments showed that when layers of salt and sugar solution are superimposed with a diffusive interface between, interfacial waves were spontaneously generated by the convection once the system evolved to a critical value of the density-anomaly ratio $R_{\rho} \equiv \beta \Delta S / \alpha \Delta T$. The waves corresponded to a local thickening of the interface and gave rise to horizontal variations in the interfacial buoyancy flux, which in turn organized otherwise random convective motions by forcing circulations on the scale of the distance between waves. Subsequent interactions between the waves and convection led to a rapid decrease in the number of waves and an increase in the scale of the convective circulations. In a rectangular channel the long-term flow consisted of a single wave which propagated back-and-forth along the channel, organizing the convection in each layer into two cells which oscillated in length from zero to the full length of the channel. However, reflection from endwalls was not required, with experiments in an annulus revealing waves and convection cells which travelled in a continuous circular path. In both 
cases, the dependence of wavespeed on the layer properties and tank dimensions was successfully predicted by assuming that effective coupling requires a matching of the wave and convection speeds, and that the system selects waves of an amplitude for which this resonance can occur.

The double-diffusive flow in a rectangular channel bears some resemblance to the El Niño-Southern Oscillation phenomenon (figure 1.1), in that for both systems there is an oscillatory change in large-scale convective circulations when waves reflect back-and-forth along a channel. There is, however, an important difference between these two systems. In the El Niño-Southern Oscillation the large heat and water vapour fluxes in the western Pacific strengthen the westward winds, which in turn lead to larger sea surface temperature gradients and larger fluxes. This 'positive feedback' tends to maintain the normal state of the system and thus perturbations are required to reverse the circulations. In contrast, the double-diffusive system exhibited 'negative feedback' because when the wave was at one end of the channel the buoyancy flux opposed the existing circulations, and eventually the circulations were forced to reverse.

Of greater significance is the similarity between the flow in an annulus and an atmospheric squall line (figure 1.2). Indeed, despite differences in the source of the convection, in both cases a large-amplitude wave propagates in one direction and strong buoyancy flux from the wave-front drives two large-scale convection cells which travel with the wave. Although the exact relationship between these two systems has yet to be explored, it appears as though a detailed comparison could lead to a deeper understanding of both systems. For example, the accepted picture of squall line formation requires low-level shear to organize the flow, but the fact that waves in the double-diffusive system form out of a disorganized environment suggests that this picture should be re-evaluated.

In conclusion, the interaction of large-amplitude waves and turbulent convection may not just be important in layered double-diffusive systems but in other laboratory and geophysical flows as well. These strongly nonlinear phenomena are worthy of further attention, although tractable mathematical models are difficult to formulate and numerical models require high resolution. 


\section{References}

Akylas, T. R., \& Grimshaw, R. H. J. 1992. Solitary internal waves with oscillatory tails. J. Fluid Mech., 242, 279-298.

BATCHELOR, G. K. 1956. On steady laminar flow with closed streamlines at large Reynolds number. J. Fluid Mech., 1, 177-190.

BENJAMIN, T. B. 1966. Internal waves of finite amplitude and permanent form. $J$. Fluid Mech., 25, 241-270.

Benjamin, T. B. 1967. Internal waves of permanent form in fluids of great depth. J. Fluid Mech., 29, 559-592.

Benny, D. J. 1966. Long nonlinear waves in fluid flows. J. Math. E Phys., 45, $52-63$.

Bensimon, D., Kolodner, P., Surko, C. M., Williams, H., \& Croquette, V. 1990. Competing and coexisting dynamical states of travelling-wave convection in an annulus. J. Fluid Mech., 217, 441-467.

BJERKnes, J. 1969. Atmospheric teleconnections from the equatorial Pacific. Mon. Weather Rev., 97, 163-172.

Christie, D. K., Muirhead, K. J., \& Hales, A. L. 1978. On solitary waves in the atmosphere. J. Atmos. Sci., 35, 805-825.

Christie, D. R. 1992. The morning glory of the Gulf of Carpentaria: a paradigm for non-linear waves in the lower atmosphere. Aust. Met. Mag., 41, 21-60.

CRAPPER, P. F. 1973. An experimental study of mixing across density interfaces. Ph.D. thesis, University of Cambridge.

Davis, R. E., \& Acrivos, A. 1967. Solitary internal waves in deep water. J. Fluid Mech., 29, 593-607.

Deane, A. E., KNobloch, E., \& Toomre, J. 1987. Traveling waves and chaos in thermosolutal convection. Phys. Rev. A, 36, 2862-2869.

DubREIL-JACotin, M. L. 1937. Sur les théorèms d'existence relatifs aux ondes permanentes périodiques à deux dimensions dans les liquides hétérogènes. $J$. Math. Pures Appl, 16, 43-67."

Elder, J. W. 1969. Numerical experiments with thermohaline convection. Phys. of Fluids (Supplement 2), 12, 194-197. 
GRIfFITHS, R. W., \& RUDDICK, B. R. 1980. Accurate fluxes across a salt-sugar finger interface deduced from direct density measurements. J. Fluid Mech., 99, 85-95.

HerRING, J. R. 1963. Investigation of problems in thermal convection. J. Atmos. Sci., 20, 325-338.

Howard, L. N. 1966. Convection at high Rayleigh number. Pages 1109-1115 of: Gortler, H. (ed), Proceedings of the Eleventh International Congress of Applied Mechanics. Munich, Federal Republic of Germany, August 1964: Springer-Berlin.

Hunt, J. C. R. 1984. Turbulence structure in thermal convection and shear-free boundary layers. J. Fluid Mech., 138, 161-184.

Huppert, H. E. 1971. On the stability of a series of double-diffusive layers. Deep Sea Res. A, 18, 1005-1021.

Huppert, H. E., \& Turner, J. S. 1981. Double-diffusive convection. J. Fluid Mech., 106, 209-329.

KELlEy, D. E. 1990. Fluxes through diffusive staircases: A new formulation. $J$. Geophys. Res., 65, 3365-3371.

Keulegan, G. H. 1953. Characteristics of internal solitary waves. J. of Res. National Bureau of Standards, 51, 133-140.

Knobloch, E., Deane, A. E., Toomre, J., \& Moore, D. R. 1986. Doubly diffusive waves. Contemp. Math., 56, 203-216.

Koop, C. G., \& Butler, G. 1981. An investigation of internal solitary waves in a two-fluid system. J. Fluid Mech., 112, 225-251.

Krumhansl, J. A. 1991. Unity in the science of physics. Physics Today, Month, 33-38.

Kubota, T., Ko, D. R. S., \& DobBs, L. D. 1978. Propagation of weakly nonlinear internal waves in a stratified fluid of finite depth. A. I. A. A. J. Hydronautics, 12, 157-165.

Lighthill, M. J. 1978. Waves in fluids. Cambridge University Press.

Linden, P. F. 1974. A note on the transport across a diffusive interface. Deep-Sea Res., 21, 283-287.

Linden, P. F., \& Shirtcliffe, T. G. L. 1978. The diffusive interface in doublediffusive convection. J. Fluid Mech., 87, 417-432.

Long, R. R. 1953. Some Aspects of the Flow of Stratified Fluids 1. A theoretical investigation. Tellus, $\mathbf{5}, 42-58$.

LoNG, R. R. 1956. Solitary waves in one- and two-fluid systems. Tellus, 8, 460-471.

LoNG, R. R. 1965. On the Boussinesq approximation and its role in the theory of internal waves. Tellus, 17, 46-52. 
Marmorino, G. O., \& Caldwell, D. R. 1976. Heat and salt transport through a diffusive thermohaline interface. Deep Sea Res. A, 23, 59-67.

MAXWORThy, T. 1976. Experiments on collisions between solitary waves. J. Fluid Mech., 76, 177-185.

MAXWORTHY, T. 1980. On the formation of nonlinear internal waves from the gravitational collapse of mixed regions in two and three dimensions. J. Fluid Mech., 96, 47.

MAXWORThy, T., \& REDEKopP, L. G. 1976. A solitary wave theory of the Great Red Spot and other observed features in the Jovian atmosphere. Icarus, 29, 261-271.

McDougall, T. J. 1981a. Double-diffusive convection with a non-linear equation of state: Part 1 . The accurate conservation of properties in a two-layer system. Prog. Oceanogr., 10, 71-89.

MCDougall, T. J. 1981b. Double-diffusive convection with a non-linear equation of state: Part 2. Laboratory experiments and their interpretation. Prog. Oceanogr., 10, 91-121.

Pullin, D. I., \& Grimshaw, R. H. J. 1988. Large-amplitude solitary waves at the interface between two homogeneous fluids. Phys. Fluids, 31, 3550-3559.

Ruddick, B. R., \& Shirtcliffe, T. G. L. 1979. Data for double diffusers: Physical properties of aqueous salt-sugar solutions. Deep-Sea Res., 26, 775787.

Russell, J. S. 1837. Report on waves. Tech. rept. No. 417. British Association for the Advancement of Science.

ShIRTCliffe, T. G. L. 1973. Transport and profile measurements of the diffusive interface in double diffusive convection with similar diffusivities. J. Fluid Mech, 57, 27-43.

SIMPSON, J. E. 1982. Gravity currents in the laboratory, atmosphere, and ocean. Ann. Rev. Fluid Mech., 14, 213.

Stern, M. E. 1960. The "Salt-Fountain" and Thermohaline Convection. Tellus, 12, 172-175.

TAKAO, S., \& NARUSAWA, V. 1980. An experimental study of heat and mass transfer across a diffusive interface. Intl. J. Heat Mass Transfer, 23, 12831285 .

TAYLOR, J. 1988. The fluxes across a diffusive interface at low values of the density ratio. Deep-Sea Res., 35, 555-567.

Thorpe, A. J., Miller, M. J., \& Moncrieff, M. W. 1982. Two-dimensional convection in non-constant shear: a model of mid-latitude squall lines. $Q . J$. R. Meteorol. Soc., 108, 739-762.

Tung, K., Chan, T. F., \& Kubota, T. 1982. Large amplitude internal wave of permanent form. Stud. Appl. Math., 66, 1-44. 
TURNER, J. S. 1965. The coupled turbulent transports of salt and heat across a sharp density interface. Int. J. Heat Mass Trans., 8, 759-767.

TURner, J. S. 1974. Double-diffusive convection. Ann. Rev. Fluid Mech., 6, 37-56.

Turner, J. S. 1985. Multicomponent convection. Ann. Rev. Fluid Mech., 17, 11-44.

Turner, J. S., \& Chen, C. F. 1974. Two-dimensional effects in double-diffusive convection. J. Fluid Mech., 63, 577-592.

VERONIS, G. 1965. On finite amplitude instability in thermohaline convection. $J$. Mar. Res., 23, 1-17.

VERONIS, G. 1968. Effect of a stabilizing gradient of solute on thermal convection. J. Fluid Mech., 34, 315-336.

Walden, R. W., Kolodner, P., Passner, A., \& Surko, C. M. 1985. Traveling waves and chaos in convection in binary fluid mixtures. Phys. Rev. Lett., 55, 496-499.

Weast, R. C. 1975. CRC Handbook of Chemistry and Physics. 56th edn. CRC Press.

Weidman, P. D., \& MaXWorThy, T. 1978. Experiments on strong interactions between solitary waves. J. Fluid. Mech., 85, 417-431.

ZABUSKY, N. J., \& KRUSKAL, M. D. 1965. Interaction of solitons in a collisionless plasma and the recurrence of initial states. Phys. Rev. Lett., 15, 240-243.

MAXWORTHY, T. 1983. Experiments on solitary internal Kelvin waves. J. Fluid. Mech., 129, 365-383. 